\title{
Divergent Reactivity of Nitrocyclopropanes with Huisgen Zwitterions and Facile Syntheses of 3-Alkoxy Pyrazolines and Pyrazoles
}

Changjiang Yang, Wei Liu, Zijian He, and Zhengjie He*

The State Key Laboratory of Elemento-Organic Chemistry and Collaborative Innovation Center of Chemical Science and Engineering (Tianjin), Nankai University, Tianjin 300071, P. R. China. E-mail: zhengjiehe@nankai.edu.cn

\section{Contents}

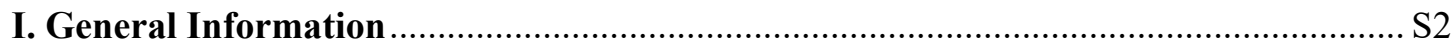

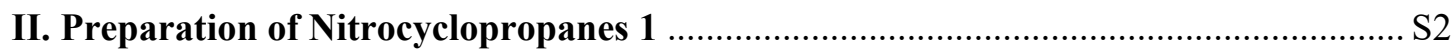

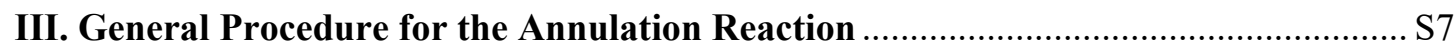

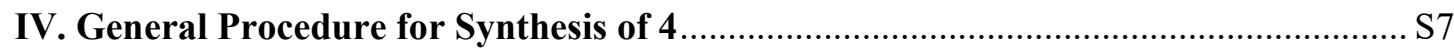

V. Control Experiments and Alternative Pathway to Intermediate E ......................... S8

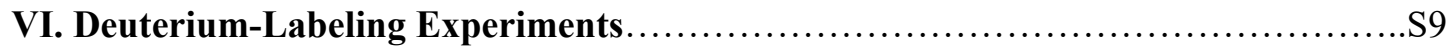

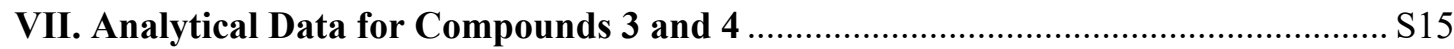

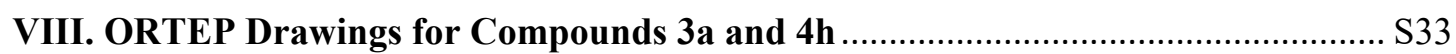

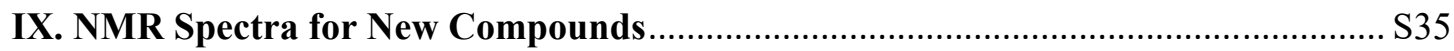




\section{General Information}

Solvents were purified prior to use according to conventional procedures. All reactions were monitored by TLC with silica gel-coated plates. Column chromatography was performed on silica gel (200-300 mesh) using a mixture of petroleum ether-ethyl acetate as the eluent. ${ }^{1} \mathrm{H}$ and ${ }^{13} \mathrm{C}$ NMR spectra were recorded in $\mathrm{CDCl}_{3}$ or DMSO- $d_{6}$ with tetramethylsilane (TMS) as the internal standard. HRMS spectra were acquired in the ESI mode (positive ion) with the mass analyzer of TOF used. Dialkyl azodicarboxylates $\mathbf{2}$ were purchased from commercial sources.

\section{Preparation of Nitrocyclopropanes 1}

The trans-2-aryl-3-nitrocyclopropane-1,1-dicarboxylates $\mathbf{1 a - 1 z ,} \mathbf{1 C}$ were prepared from the Michael adducts $\mathbf{6}$ of $\beta$-nitrostyrenes 5 with dialkyl malonates according to a literature procedure. ${ }^{1}$ The corresponding $\beta$-nitrostyrenes 5 were prepared according to previous reported procedures. ${ }^{2}$ Nitrocyclopropanes $\mathbf{1} \mathbf{A}^{3 \mathrm{a}}$ and $\mathbf{1} \mathbf{B}^{4}$ were prepared according to similar methods.

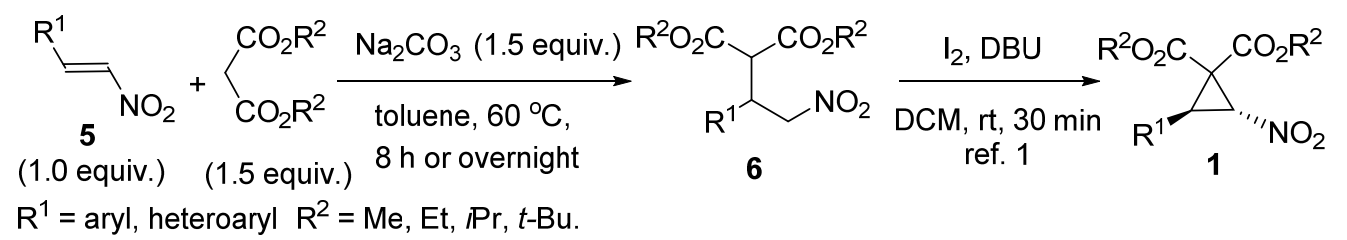

General Procedure for preparation of dimethyl trans-2-nitro-3-phenylcyclopropane-1,1dicarboxylate 1a:

To a mixture of (E)-(2-nitrovinyl)benzene $5 \mathbf{a}(1.0 \mathrm{~g}, 6.7 \mathrm{mmol})$ and $\mathrm{Na}_{2} \mathrm{CO}_{3}(1.06 \mathrm{~g}, 10.0 \mathrm{mmol})$ in toluene $(20 \mathrm{~mL})$ was added diethyl malonate $(1.52 \mathrm{~mL}, 10.0 \mathrm{mmol})$ at room temperature and the resulting mixture was stirred at $60{ }^{\circ} \mathrm{C}$ for $8 \mathrm{~h}$ or overnight. The reaction was complete (monitored by TLC) and the reaction mixture was then cooled to room temperature and directly applied to column chromatographic isolation on silica gel (petroleum ether/ethyl acetate 5:1) to give the product $\mathbf{6 a}$ as slightly brown oil $(1.82 \mathrm{~g}, 88 \%)$.

To a mixture of $6 \mathrm{a}(0.62 \mathrm{~g}, 2.0 \mathrm{mmol})$ and DBU $(0.6 \mathrm{~mL}, 4.0 \mathrm{mmol})$ in dichloromethane (DCM, $10 \mathrm{~mL})$ was added iodine $(1.02 \mathrm{~g}, 4.0 \mathrm{mmol})$ and the resulting mixture was stirred at room temperature for $30 \mathrm{~min}$. The reaction was complete (monitored by TLC) and quenched with saturated aqueous $\mathrm{Na}_{2} \mathrm{~S}_{2} \mathrm{O}_{3}(20 \mathrm{~mL})$ and extracted with DCM $(10 \mathrm{~mL})$. The aqueous phase was extracted with DCM $(10 \mathrm{~mL})$ again. The combined organic layers were washed with saturated brine $(30 \mathrm{~mL} \times 2)$, dried over anhydrous $\mathrm{Na}_{2} \mathrm{SO}_{4}$. After filtration and concentration under reduced pressure, the crude product was purified by column chromatography on silica gel (petroleum ether/ethyl acetate $20: 1)$ to give $1 \mathbf{a}$ as colorless oil $(0.54 \mathrm{~g}, 90 \%)$.

Compounds $\mathbf{1 b}-\mathbf{1 z}, \mathbf{1 C}$ were prepared by following above general procedure for $\mathbf{1 a}$. 


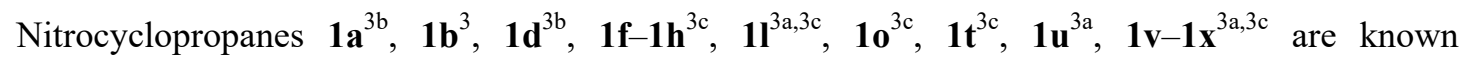
compounds.

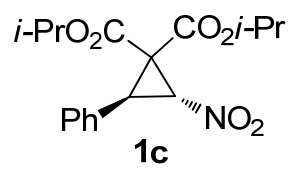

White solid, mp $70-71{ }^{\circ} \mathrm{C}$; $0.56 \mathrm{~g}, 84 \%$ yield in the final step; ${ }^{1} \mathrm{H}$ NMR $\left(400 \mathrm{MHz}, \mathrm{CDCl}_{3}\right): \delta$ 7.34-7.27 (m, 5H), 5.39 (d, $J=5.9 \mathrm{~Hz}, 1 \mathrm{H}), 5.20-5.11(\mathrm{~m}, 1 \mathrm{H}), 4.87-4.78(\mathrm{~m}, 1 \mathrm{H}), 4.18$ (d, $J=$ $5.9 \mathrm{~Hz}, 1 \mathrm{H}), 1.31(\mathrm{~d}, J=6.2 \mathrm{~Hz}, 3 \mathrm{H}), 1.25(\mathrm{~d}, J=6.2 \mathrm{~Hz}, 3 \mathrm{H}), 1.01(\mathrm{~d}, J=6.2 \mathrm{~Hz}, 3 \mathrm{H}), 0.98(\mathrm{~d}, J$ $=6.2 \mathrm{~Hz}, 3 \mathrm{H}) \mathrm{ppm} ;{ }^{13} \mathrm{C} \mathrm{NMR}\left(100 \mathrm{MHz}, \mathrm{CDCl}_{3}\right): \delta 162.7,162.6,130.3,128.6,128.4,128.3,70.7$, 66.2, 46.4, 37.3, 21.4, 21.1 ppm; HRMS-ESI calcd for $\mathrm{C}_{17} \mathrm{H}_{22} \mathrm{NO}_{6}[\mathrm{M}+\mathrm{H}]^{+}$336.1442, found 336.1443 .

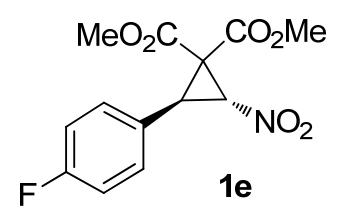

Yellow oil; $0.48 \mathrm{~g}, 81 \%$ yield in the final step; ${ }^{1} \mathrm{H}$ NMR (400 MHz, $\left.\mathrm{CDCl}_{3}\right): \delta 7.27-7.24(\mathrm{~m}, 2 \mathrm{H})$, 7.03 (t, $J=8.3 \mathrm{~Hz}, 2 \mathrm{H}), 5.37$ (d, $J=5.9 \mathrm{~Hz}, 1 \mathrm{H}), 4.16(\mathrm{~d}, J=5.9 \mathrm{~Hz}, 1 \mathrm{H}), 3.85$ (s, 3H), 3.57 (s, $3 \mathrm{H}) \mathrm{ppm} ;{ }^{13} \mathrm{C} \mathrm{NMR}\left(100 \mathrm{MHz}, \mathrm{CDCl}_{3}\right): \delta 163.6,163.4,162.7$ (d, $\left.J=248.3 \mathrm{~Hz}\right), 130.0(\mathrm{~d}, J=8.5$ $\mathrm{Hz}), 125.8$ (d, $J=3.1 \mathrm{~Hz}), 115.9$ (d, $J=21.9 \mathrm{~Hz}), 66.2,53.9,53.5,45.9,36.9 \mathrm{ppm} ;{ }^{19} \mathrm{~F}$ NMR $(376$ $\mathrm{MHz}, \mathrm{CDCl}_{3}$ ): $\delta-112.5$ ppm; HRMS-ESI calcd for $\mathrm{C}_{13} \mathrm{H}_{12} \mathrm{FNNaO}_{6}[\mathrm{M}+\mathrm{Na}]^{+} 320.0541$, found 320.0541 .

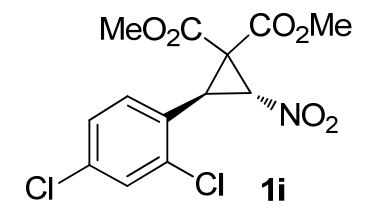

Slightly yellow solid, mp $88-89{ }^{\circ} \mathrm{C}$; $0.61 \mathrm{~g}, 88 \%$ yield in the final step; ${ }^{1} \mathrm{H}$ NMR $(400 \mathrm{MHz}$, $\left.\mathrm{CDCl}_{3}\right): \delta 7.43(\mathrm{~d}, J=2.1 \mathrm{~Hz}, 1 \mathrm{H}), 7.25(\mathrm{dd}, J=8.4,2.1 \mathrm{~Hz}, 1 \mathrm{H}), 7.15(\mathrm{~d}, J=8.4 \mathrm{~Hz}, 1 \mathrm{H}), 5.31(\mathrm{~d}$, $J=6.0 \mathrm{~Hz}, 1 \mathrm{H}), 4.14(\mathrm{~d}, J=6.0 \mathrm{~Hz}, 1 \mathrm{H}), 3.86(\mathrm{~s}, 3 \mathrm{H}), 3.65(\mathrm{~s}, 3 \mathrm{H}) \mathrm{ppm} ;{ }^{13} \mathrm{C} \mathrm{NMR}(100 \mathrm{MHz}$, $\left.\mathrm{CDCl}_{3}\right): \delta 163.8,163.2,135.9,135.4,130.5,129.8,127.3,127.0,66.6,53.9,53.7,45.2,35.8$ ppm; HRMS-ESI calcd for $\mathrm{C}_{13} \mathrm{H}_{11} \mathrm{Cl}_{2} \mathrm{NNaO}_{6}[\mathrm{M}+\mathrm{Na}]^{+}$369.9856, found 369.9859 .

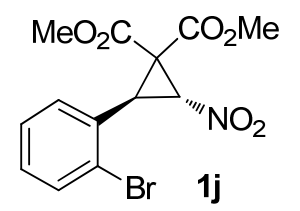

Slightly yellow oil; $0.57 \mathrm{~g}, 80 \%$ yield in the final step; ${ }^{1} \mathrm{H}$ NMR $\left(400 \mathrm{MHz}, \mathrm{CDCl}_{3}\right): \delta 7.59(\mathrm{~d}, J=$ $7.9 \mathrm{~Hz}, 1 \mathrm{H}), 7.30$ (t, $J=7.5 \mathrm{~Hz}, 1 \mathrm{H}), 7.22$ (t, $J=7.9 \mathrm{~Hz}, 1 \mathrm{H}), 7.18$ (d, $J=8.0 \mathrm{~Hz}, 1 \mathrm{H}), 5.33$ (d, $J$ 
$=6.0 \mathrm{~Hz}, 1 \mathrm{H}), 4.17(\mathrm{~d}, J=6.0 \mathrm{~Hz}, 1 \mathrm{H}), 3.86(\mathrm{~s}, 3 \mathrm{H}), 3.63(\mathrm{~s}, 3 \mathrm{H}) \mathrm{ppm} ;{ }^{13} \mathrm{C}$ NMR $(100 \mathrm{MHz}$, $\left.\mathrm{CDCl}_{3}\right): \delta 163.8,163.4,133.2,130.2,130.1,129.8,127.4,124.9,67.2,53.8,53.5,45.5,38.5 \mathrm{ppm}$; HRMS-ESI calcd for $\mathrm{C}_{13} \mathrm{H}_{12} \mathrm{BrNNaO}_{6}[\mathrm{M}+\mathrm{Na}]^{+} 379.9740$, found 379.9740 .

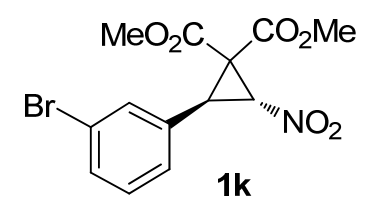

Slightly yellow oil; $0.65 \mathrm{~g}, 90 \%$ yield in the final step; ${ }^{1} \mathrm{H}$ NMR (400 MHz, $\left.\mathrm{CDCl}_{3}\right): \delta 7.47-7.44$ (m, 2H), 7.24-7.19 (m, 2H), 5.37 (d, $J=6.0 \mathrm{~Hz}, 1 \mathrm{H}), 4.16$ (d, $J=6.0 \mathrm{~Hz}, 1 \mathrm{H}), 3.85$ (s, 3H), 3.60 (s, 3H) ppm; ${ }^{13} \mathrm{C}$ NMR $\left(100 \mathrm{MHz}, \mathrm{CDCl}_{3}\right): \delta 163.5,163.3,132.3,131.8,131.4,130.3,126.8$, 122.7, 65.9, 54.0, 53.5, 45.8, 36.8 ppm; HRMS-ESI calcd for $\mathrm{C}_{13} \mathrm{H}_{12} \mathrm{BrNNaO}_{6}[\mathrm{M}+\mathrm{Na}]^{+}$ 379.9740 , found 379.9738 .

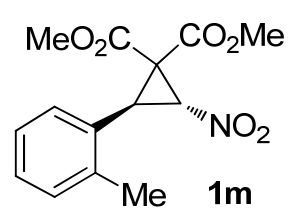

White solid, mp $74-75{ }^{\circ} \mathrm{C} ; 0.45 \mathrm{~g}, 77 \%$ yield in the final step; ${ }^{1} \mathrm{H}$ NMR $\left(400 \mathrm{MHz}, \mathrm{CDCl}_{3}\right): \delta$ 7.26-7.14 (m, 3H), 7.12 (d, $J=7.4 \mathrm{~Hz}, 1 \mathrm{H}), 5.44(\mathrm{~d}, J=6.0 \mathrm{~Hz}, 1 \mathrm{H}), 4.12(\mathrm{~d}, J=6.0 \mathrm{~Hz}, 1 \mathrm{H})$, $3.85(\mathrm{~s}, 3 \mathrm{H}), 3.52(\mathrm{~s}, 3 \mathrm{H}), 2.43(\mathrm{~s}, 3 \mathrm{H}) \mathrm{ppm} ;{ }^{13} \mathrm{C} \mathrm{NMR}\left(100 \mathrm{MHz}, \mathrm{CDCl}_{3}\right): \delta 163.9,163.7,138.1$, 130.5, 128.7, 128.1, 127.7, 125.9, 66.2, 53.8, 53.3, 45.6, 37.1, 19.1 ppm; HRMS-ESI calcd for $\mathrm{C}_{14} \mathrm{H}_{15} \mathrm{NNaO}_{6}[\mathrm{M}+\mathrm{Na}]^{+} 316.0792$, found 316.0793 .

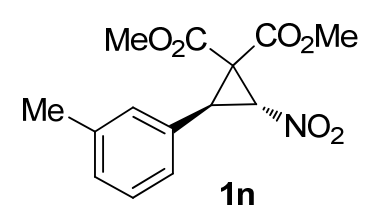

Slightly yellow oil; $0.50 \mathrm{~g}, 86 \%$ yield in the final step; ${ }^{1} \mathrm{H} \mathrm{NMR}\left(400 \mathrm{MHz}, \mathrm{CDCl}_{3}\right): \delta 7.21$ (t, $J=$ $7.6 \mathrm{~Hz}, 1 \mathrm{H}), 7.12$ (d, $J=7.6 \mathrm{~Hz}, 1 \mathrm{H}), 7.08$ (s, 1H), 7.04 (d, $J=7.6 \mathrm{~Hz}, 1 \mathrm{H}), 5.39$ (d, $J=6.0 \mathrm{~Hz}$, $1 \mathrm{H}), 4.16(\mathrm{~d}, J=6.0 \mathrm{~Hz}, 1 \mathrm{H}), 3.85(\mathrm{~s}, 3 \mathrm{H}), 3.56(\mathrm{~s}, 3 \mathrm{H}), 2.33$ (s, 3H) ppm; ${ }^{13} \mathrm{C}$ NMR (100 MHz, $\left.\mathrm{CDCl}_{3}\right): \delta 163.8,163.5,138.6,129.9,129.3,128.9,128.6,125.0,66.2,53.8,53.3,46.0,37.7,21.2$ ppm; HRMS-ESI calcd for $\mathrm{C}_{14} \mathrm{H}_{15} \mathrm{NNaO}_{6}[\mathrm{M}+\mathrm{Na}]^{+}$316.0792, found 316.0794.

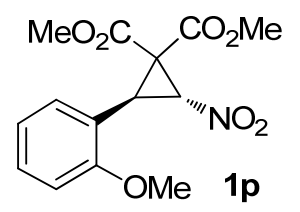

White solid, mp $119-120{ }^{\circ} \mathrm{C} ; 0.53 \mathrm{~g}, 86 \%$ yield in the final step; ${ }^{1} \mathrm{H}$ NMR $\left(400 \mathrm{MHz}, \mathrm{CDCl}_{3}\right): \delta$ $7.32-7.27(\mathrm{~m}, 1 \mathrm{H}), 7.09$ (d, $J=7.5 \mathrm{~Hz}, 1 \mathrm{H}), 6.91$ (t, $J=7.5 \mathrm{~Hz}, 1 \mathrm{H}), 6.87$ (d, $J=8.3 \mathrm{~Hz}, 1 \mathrm{H})$, $5.27(\mathrm{~d}, J=6.1 \mathrm{~Hz}, 1 \mathrm{H}), 4.08(\mathrm{~d}, J=6.1 \mathrm{~Hz}, 1 \mathrm{H}), 3.84(\mathrm{~s}, 3 \mathrm{H}), 3.83(\mathrm{~s}, 3 \mathrm{H}), 3.59(\mathrm{~s}, 3 \mathrm{H}) \mathrm{ppm} ;{ }^{13} \mathrm{C}$ 
NMR (100 MHz, $\left.\mathrm{CDCl}_{3}\right): \delta 164.2,164.0,158.1,129.9,128.9,120.4,118.6,110.6,67.0,55.5$, 53.7, 53.2, 45.2, 34.1 ppm; HRMS-ESI calcd for $\mathrm{C}_{14} \mathrm{H}_{15} \mathrm{NNaO}_{7}[\mathrm{M}+\mathrm{Na}]^{+}$332.0741, found 332.0743 .

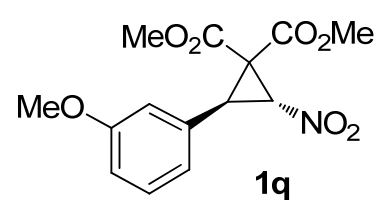

Yellow solid, mp $63-64{ }^{\circ} \mathrm{C} ; 0.54 \mathrm{~g}, 87 \%$ yield in the final step; ${ }^{1} \mathrm{H}$ NMR $\left(400 \mathrm{MHz}, \mathrm{CDCl}_{3}\right): \delta$ $7.24(\mathrm{t}, J=8.0 \mathrm{~Hz}, 1 \mathrm{H}), 6.86-6.82(\mathrm{~m}, 2 \mathrm{H}), 6.79(\mathrm{~s}, 1 \mathrm{H}), 5.38(\mathrm{~d}, J=5.9 \mathrm{~Hz}, 1 \mathrm{H}), 4.17$ (d, $J=5.9$ $\mathrm{Hz}, 1 \mathrm{H}), 3.85$ (s, 3H), 3.79 (s, 3H), $3.58(\mathrm{~s}, 3 \mathrm{H}) \mathrm{ppm} ;{ }^{13} \mathrm{C}$ NMR (100 MHz, $\left.\mathrm{CDCl}_{3}\right): \delta 163.7$, 163.5, 159.8, 131.5, 129.8, 120.3, 114.1, 113.8, 66.2, 55.2, 53.9, 53.4, 46.0, 37.6 ppm; HRMS-ESI calcd for $\mathrm{C}_{14} \mathrm{H}_{15} \mathrm{NNaO}_{7}[\mathrm{M}+\mathrm{Na}]^{+} 332.0741$, found 332.0748 .

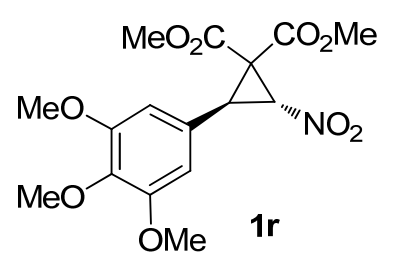

White solid, mp $146-148{ }^{\circ} \mathrm{C} ; 0.65 \mathrm{~g}, 88 \%$ yield in the final step; ${ }^{1} \mathrm{H}$ NMR $\left(400 \mathrm{MHz}, \mathrm{CDCl}_{3}\right): \delta$ $6.44(\mathrm{~s}, 2 \mathrm{H}), 5.38(\mathrm{~d}, J=5.9 \mathrm{~Hz}, 1 \mathrm{H}), 4.15(\mathrm{~d}, J=5.9 \mathrm{~Hz}, 1 \mathrm{H}), 3.86(\mathrm{~s}, 3 \mathrm{H}), 3.85$ (s, 6H), 3.83 (s, $3 \mathrm{H}), 3.62$ (s, 3H) ppm; ${ }^{13} \mathrm{C} \mathrm{NMR}\left(100 \mathrm{MHz}, \mathrm{CDCl}_{3}\right): \delta 163.7,163.5,153.4,138.1,125.5,105.1$, 66.4, 60.8, 56.2, 56.1, 53.9, 53.5, 46.2, 37.9 ppm; HRMS-ESI calcd for $\mathrm{C}_{16} \mathrm{H}_{19} \mathrm{NNaO}_{9}[\mathrm{M}+\mathrm{Na}]^{+}$ 392.0952, found 392.0948 .

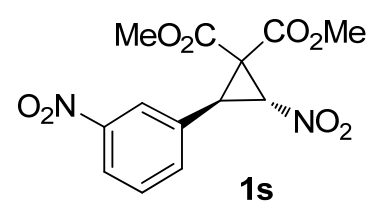

Yellow solid, mp $121-122{ }^{\circ} \mathrm{C}$; $0.53 \mathrm{~g}, 82 \%$ yield in the final step; ${ }^{1} \mathrm{H}$ NMR $\left(400 \mathrm{MHz}, \mathrm{CDCl}_{3}\right): \delta$ $8.20(\mathrm{~d}, J=8.1 \mathrm{~Hz}, 1 \mathrm{H}), 8.17$ (s, 1H), 7.67 (d, $J=7.9 \mathrm{~Hz}, 1 \mathrm{H}), 7.58$ (t, $J=7.9 \mathrm{~Hz}, 1 \mathrm{H}), 5.47$ (d, $J$ $=5.9 \mathrm{~Hz}, 1 \mathrm{H}), 4.28(\mathrm{~d}, J=5.9 \mathrm{~Hz}, 1 \mathrm{H}), 3.88(\mathrm{~s}, 3 \mathrm{H}), 3.62(\mathrm{~s}, 3 \mathrm{H}) \mathrm{ppm} ;{ }^{13} \mathrm{C} \mathrm{NMR}(100 \mathrm{MHz}$, $\left.\mathrm{CDCl}_{3}\right): \delta 163.3,163.2,148.2,134.5,132.2,129.9,123.6,123.3,65.8,54.0,53.7,45.7,36.5$ ppm; HRMS-ESI calcd for $\mathrm{C}_{13} \mathrm{H}_{12} \mathrm{~N}_{2} \mathrm{NaO}_{8}[\mathrm{M}+\mathrm{Na}]^{+}$347.0486, found 347.0491 .

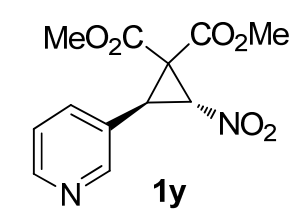

Slightly yellow solid, mp $89-91{ }^{\circ} \mathrm{C} ; 0.40 \mathrm{~g}, 72 \%$ yield in the final step; ${ }^{1} \mathrm{H}$ NMR $(400 \mathrm{MHz}$, $\left.\mathrm{CDCl}_{3}\right): \delta 8.60-8.57(\mathrm{~m}, 2 \mathrm{H}), 7.60(\mathrm{~d}, J=7.9 \mathrm{~Hz}, 1 \mathrm{H}), 7.30(\mathrm{dd}, J=7.8,5.0 \mathrm{~Hz}, 1 \mathrm{H}), 5.42(\mathrm{~d}, J=$ 
$6.0 \mathrm{~Hz}, 1 \mathrm{H}), 4.18(\mathrm{~d}, J=6.0 \mathrm{~Hz}, 1 \mathrm{H}), 3.87$ (s, 3H), 3.60 (s, 3H) ppm; ${ }^{13} \mathrm{C}$ NMR $(100 \mathrm{MHz}$, $\left.\mathrm{CDCl}_{3}\right): \delta 163.3,163.3,149.9,149.7,135.8,126.1,123.4,65.6,54.1,53.7,45.6,35.0$ ppm; HRMS-ESI calcd for $\mathrm{C}_{12} \mathrm{H}_{13} \mathrm{~N}_{2} \mathrm{O}_{6}[\mathrm{M}+\mathrm{H}]^{+} 281.0768$, found 281.0770 .

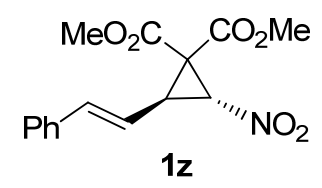

Colorless oil, ${ }^{1} \mathrm{H}$ NMR $\left(400 \mathrm{MHz}, \mathrm{CDCl}_{3}\right): \delta$ 7.34-7.25 (m, 5H), $6.84(\mathrm{~d}, J=15.9 \mathrm{~Hz}, 1 \mathrm{H}), 5.91$ (dd, $J=15.9,8.8 \mathrm{~Hz}, 1 \mathrm{H}), 5.12$ (d, $J=5.5 \mathrm{~Hz}, 1 \mathrm{H}), 3.81$ (s, 3H), 3.80 (s, 3H), 3.68 (dd, $J=8.8$, $5.5 \mathrm{~Hz}, 1 \mathrm{H}) \mathrm{ppm} ;{ }^{13} \mathrm{C} \mathrm{NMR}\left(100 \mathrm{MHz}, \mathrm{CDCl}_{3}\right): \delta 164.4,163.5,137.7,135.5,128.7,128.5,126.4$, 117.7, 67.4, 53.8, 53.7, 45.0, 36.9 ppm; HRMS-ESI calcd for $\mathrm{C}_{15} \mathrm{H}_{15} \mathrm{NNaO}_{6}[\mathrm{M}+\mathrm{Na}]^{+}$328.0792, found 328.0796 .

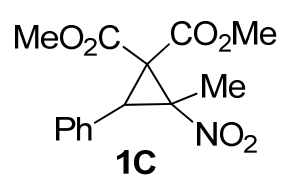

Colorless oil, $\mathrm{dr}$ 6:1, NMR data for the major isomer: ${ }^{1} \mathrm{H}$ NMR (400 $\left.\mathrm{MHz}, \mathrm{CDCl}_{3}\right): \delta 7.38-7.33$ (m, 3H), 7.22-7.19 (m, 2H), $4.28(\mathrm{~s}, 1 \mathrm{H}), 3.82$ (s, 3H), 3.74 (s, 3H), 1.98 (s, 3H) ppm; ${ }^{13} \mathrm{C} \mathrm{NMR}$ $\left(100 \mathrm{MHz}, \mathrm{CDCl}_{3}\right): \delta 165.8,164.0,130.3,128.9,128.7,128.2,74.4,53.9,53.1,45.7,38.9,14.7$ ppm; selected NMR data for the minor isomer: ${ }^{1} \mathrm{H}$ NMR (400 $\mathrm{MHz}, \mathrm{CDCl}_{3}$ ): $\delta 7.32-7.29(\mathrm{~m})$, 3.89 (s), 3.67 (s), 2.06 (s) ppm; ${ }^{13} \mathrm{C}$ NMR (100 MHz, $\left.\mathrm{CDCl}_{3}\right): \delta 166.7,163.8,130.1,129.0,128.2$, 127.7, 71.9, 54.1, 53.0, 46.2, 41.8, 16.5 ppm; HRMS-ESI calcd for $\mathrm{C}_{14} \mathrm{H}_{16} \mathrm{NO}_{6}[\mathrm{M}+\mathrm{H}]^{+}$294.0972, found 294.0975.

\section{References}

1. Selvi, T.; Srinivasan, K. J. Org. Chem. 2014, 79, 3653-3658.

2. (a) Xia, X.-F.; Shu, X.-Z.; Ji, K.-G.; Yang, Y.-F.; Shaukat, A.; Liu, X.-Y.; Liang, Y.-M. J. Org. Chem. 2010, 75, 2893-2902; (b) Kuster, G. J. T.; Steeghs, R. H. J.; Scheeren, H. W. Eur. J. Org. Chem. 2001, 553-560; (c) Quan, X.-J.; Ren, Z.-H.; Wang, Y.-Y.; Guan, Z.-H. Org. Lett. 2014, 16, 5728-5731.

3. (a) McCooey, S. H.; McCabe, T.; Connon, S. J. J. Org. Chem. 2006, 71, 7494-7497; (b) Fan, R.-H.; Ye, Y.; Li, W.-S.; Wang, L.-F. Adv. Synth. Catal. 2008, 350, 2488-2492; (c) Y.-N. Xuan, S.-Z. Nie, L-T. Dong, J.-M. Zhang and M. Yan, Org. Lett. 2009, 11, 1583-1586.

4. Goldberg, A. F. G.; O’Connor, N. R.; Craig II, R. A.; Stoltz, B. M. Org. Lett. 2012, 14, 5314-5317. 


\section{General Procedure for the Annulation Reaction}

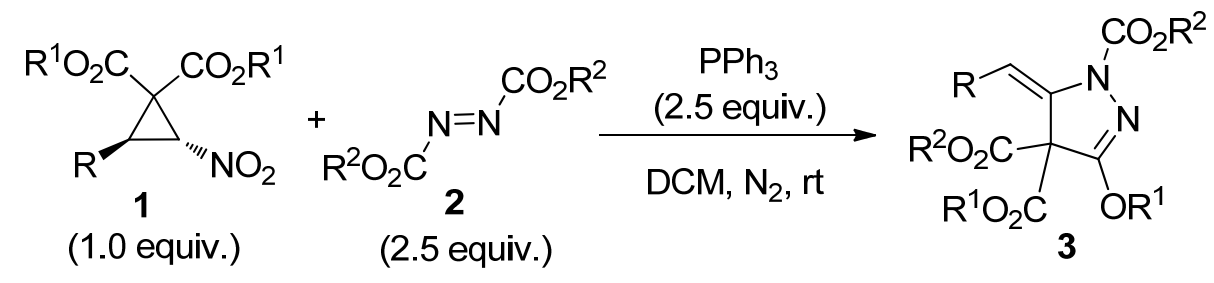

Under a $\mathrm{N} 2$ atmosphere, to a solution of nitrocyclopropane $1(0.30 \mathrm{mmol})$ and dialkyl azodicarboxylate $2(0.75 \mathrm{mmol})$ in $\mathrm{CH}_{2} \mathrm{Cl}_{2}(2.0 \mathrm{~mL})$ was added $\mathrm{Ph}_{3} \mathrm{P}(197 \mathrm{mg}, 0.75 \mathrm{mmol})$. The resulting mixture was stirred at room temperature for a specified time. After the reaction was complete as monitored by TLC, the solvent was removed on a rotary evaporator under reduced pressure, and the residue was subjected to column chromatographic isolation on silica gel (gradient eluent: petroleum ether/ethyl acetate 15:1-10:1) to give compound 3 .

\section{General Procedure for Synthesis of 4}

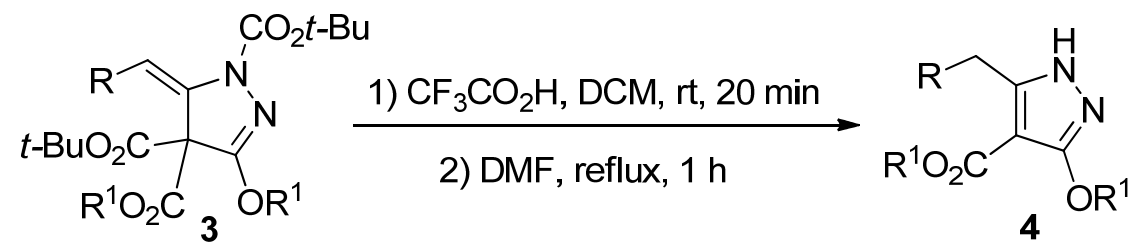

To a solution of $3(0.2 \mathrm{mmol})$ in $\mathrm{CH}_{2} \mathrm{Cl}_{2}(2.0 \mathrm{~mL})$ was added trifluoroacetic acid $(0.2 \mathrm{~mL})$ via a syringe and the mixture was stirred at room temperature for $20 \mathrm{~min}$. Then the solvent and other volatile components were removed on a rotary evaporator under reduced pressure. The residue was dissolved in $\mathrm{N}, \mathrm{N}$-dimethylformamide (DMF, $2.0 \mathrm{~mL}$ ) and the resulting mixture was stirred at reflux for $1 \mathrm{~h}$. After being cooled to room temperature, the reaction mixture was diluted with water $(5 \mathrm{~mL})$ and extracted with EtOAc $(5 \mathrm{~mL} \times 2)$. The combined organic layers were washed with saturated brine $(10 \mathrm{~mL} \times 3)$, dried over anhydrous $\mathrm{MgSO}_{4}$. After filtration and concentration on a rotary evaporator, the residue was purified by column chromatography on silica gel (gradient eluent: petroleum ether/ethyl acetate 5:1-3:1) to afford pyrazole 4 . 


\section{Control Experiments and Alternative Pathway to Intermediate E}

\section{Control Experiments}

(1)

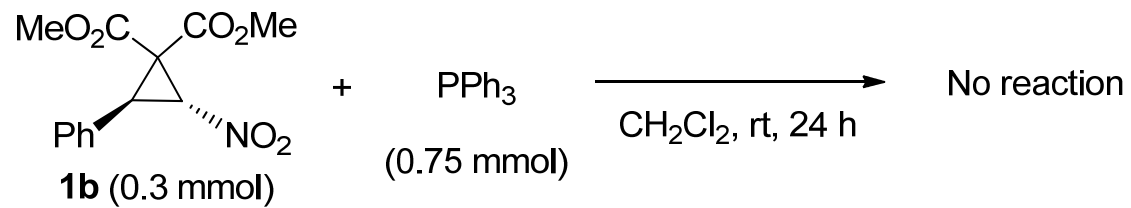

(2)

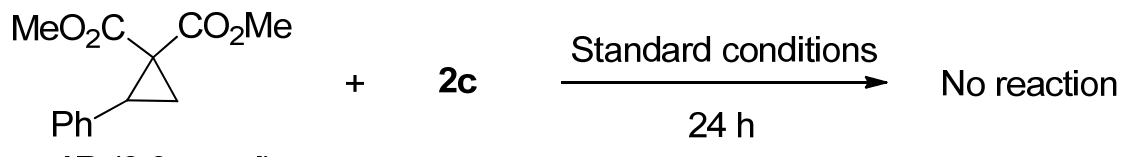

(3)

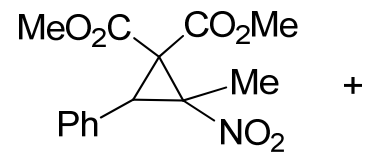
2c

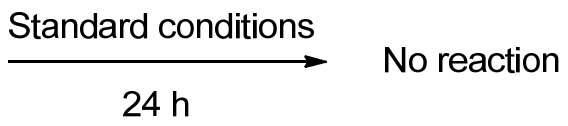

$1 \mathrm{C}(\mathrm{dr}=6: 1,0.3 \mathrm{mmol})$

\section{Alternative Pathway to Intermediate E}

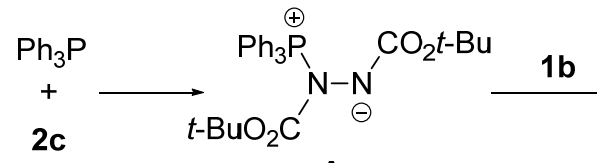

A<smiles>COC(=O)C1(C(C)=O)CC1c1ccccc1[N+](=O)[O-]</smiles>

$B^{\prime}$
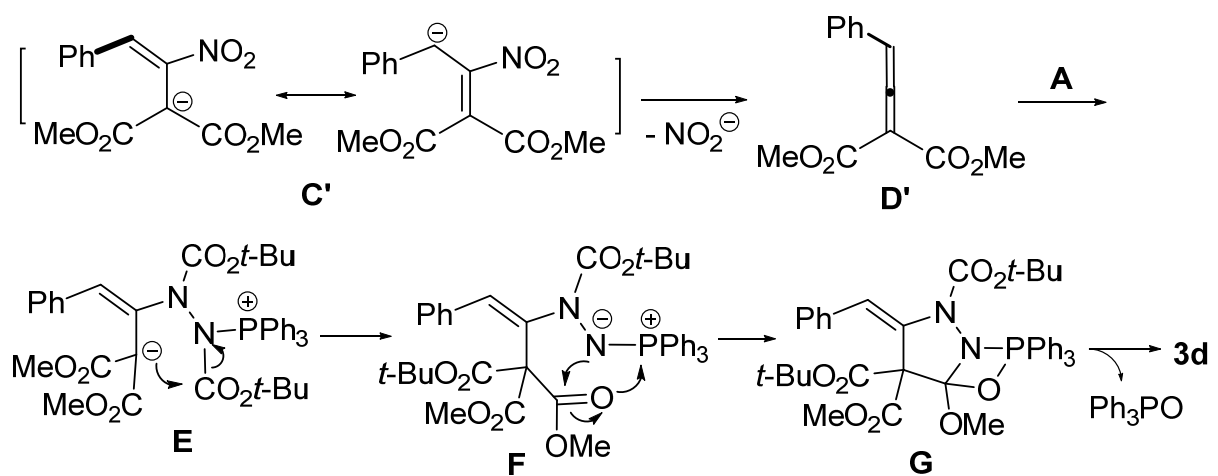

Owing to the relatively poor leaving group ability of nitro group, the above possible pathway to intermediate $\mathbf{E}$ should be also taken into consideration. In this alternative pathway, the in situ generated Huisgen zwitterion A first acts as a base to deprotonate nitrocyclopropane $\mathbf{1 b}$ producing intermediate B'. B' undergoes a ring-opening rearrangement to generate resonance-stabilizing allylic carbanion $\mathbf{C}^{\prime}$, which subsequently experiences elimination of nitro group to yield allene intermediate D'. D' is then captured by zwitterion A through a nucleophilic addition to generate intermediate E. By this way, the substitution of nitro group can be well circumvented. 


\section{Deuterium-Labeling Experiments}

\section{The Reaction of Deuterated Nitrocyclopropane 1h- $d_{1}$ with Huisgen Zwitterion}

(a) Preparation of deuterated nitrocyclopropane $\mathbf{1} \mathbf{h}-d_{1}$

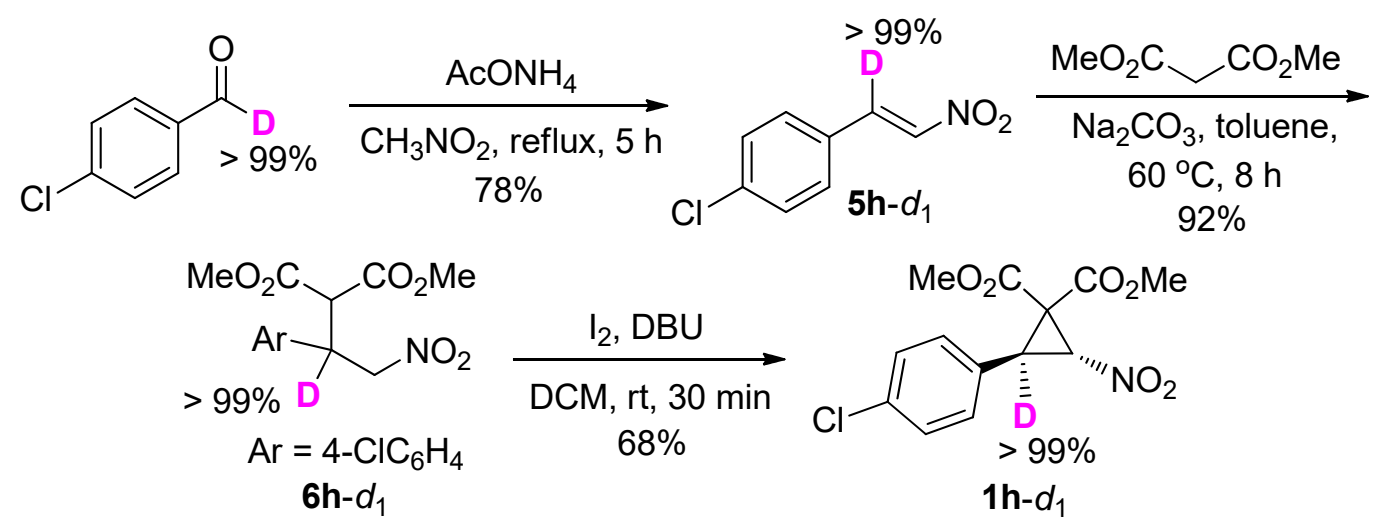

Deuterated 4-chlorobenzaldehyde was prepared according to the reported procedure, ${ }^{5}$ and the analytical data were in accordance with those in the literature report. ${ }^{1} \mathrm{H}$ NMR $\left(400 \mathrm{MHz}, \mathrm{CDCl}_{3}\right)$ : $\delta 7.84(\mathrm{~d}, J=8.4 \mathrm{~Hz}, 2 \mathrm{H}), 7.53(\mathrm{~d}, J=8.4 \mathrm{~Hz}, 2 \mathrm{H}) \mathrm{ppm}$.

Compound $5 \mathbf{h}-d_{1}$. To a solution of deuterated 4-chlorobenzaldehyde $(0.60 \mathrm{~g}, 4.20 \mathrm{mmol})$ in nitromethane $(10 \mathrm{~mL})$ was added ammonium acetate $(81.0 \mathrm{mg}, 1.05 \mathrm{mmol})$ and the resulting mixture was stirred at reflux for $5 \mathrm{~h}$. Excessive nitromethane was then removed on a rotary evaporator under reduced pressure, and the residue was mixed into water $(10 \mathrm{~mL})$ and extracted with $\mathrm{CH}_{2} \mathrm{Cl}_{2}(10 \mathrm{~mL} \times 2)$. The combined organic layers were washed with saturated brine $(20 \mathrm{~mL}$ $\times 2$ ) and dried over anhydrous $\mathrm{MgSO}_{4}$. After filtration and concentration under reduced pressure, the crude product was purified by column chromatography on silica gel (eluent: petroleum ether/ethyl acetate 50:1) to give $\mathbf{5 h}-d_{1}$ as yellow solid, $0.62 \mathrm{~g}$, yield $78 \%$, mp $110-112{ }^{\circ} \mathrm{C} ;{ }^{1} \mathrm{H}$ NMR (400 MHz, $\left.\mathrm{CDCl}_{3}\right): \delta 7.56(\mathrm{~s}, 1 \mathrm{H}), 7.50(\mathrm{~d}, J=8.7 \mathrm{~Hz}, 2 \mathrm{H}), 7.44(\mathrm{~d}, J=8.7 \mathrm{~Hz}, 2 \mathrm{H}) \mathrm{ppm}$.

Compound $6 \mathbf{h}-d_{1}$. To a mixture of $\mathbf{5 h}-d_{1}(554 \mathrm{mg}, 3.0 \mathrm{mmol})$ and $\mathrm{Na}_{2} \mathrm{CO}_{3}(477 \mathrm{mg}, 4.5 \mathrm{mmol})$ in toluene $(10 \mathrm{~mL})$ was added dimethyl malonate $(514 \mu \mathrm{L}, 4.5 \mathrm{mmol})$, and the resulting mixture was then stirred at $60^{\circ} \mathrm{C}$ for $8 \mathrm{~h}$. After the reaction was complete (monitored by TLC), the reaction mixture was cooled to room temperature and directly applied to column chromatographic isolation on silica gel (petroleum ether/ethyl acetate 5:1) to give $\mathbf{6 h}-d_{1}$ as slightly yellow oil, $874 \mathrm{mg}$, yield 92\%; ${ }^{1} \mathrm{H}$ NMR (400 MHz, $\left.\mathrm{CDCl}_{3}\right): \delta 7.31(\mathrm{~d}, J=8.6 \mathrm{~Hz}, 2 \mathrm{H}), 7.18(\mathrm{~d}, J=8.6 \mathrm{~Hz}, 2 \mathrm{H}), 4.91$ (d, $J=$ $13.3 \mathrm{~Hz}, 1 \mathrm{H}), 4.84(\mathrm{~d}, J=13.3 \mathrm{~Hz}, 1 \mathrm{H}), 3.82(\mathrm{~s}, 1 \mathrm{H}), 3.77$ (s, 3H), 3.59 (s, 3H) ppm.

Compound $\mathbf{1 h}-d_{1}$. To a mixture of $\mathbf{6 h}-d_{1}(369 \mathrm{mg}, 2 \mathrm{mmol})$ and $\mathrm{DBU}(0.6 \mathrm{~mL}, 4 \mathrm{mmol})$ in DCM $(10 \mathrm{~mL})$ was added iodine $(1.02 \mathrm{~g}, 4 \mathrm{mmol})$, and the resulting mixture was stirred at $\mathrm{rt}$ for $30 \mathrm{~min}$. The reaction was complete (monitored by TLC) and quenched with saturated aqueous $\mathrm{Na}_{2} \mathrm{~S}_{2} \mathrm{O}_{3}(20 \mathrm{~mL})$ and extracted with DCM $(10 \mathrm{~mL})$. The aqueous phase was extracted with DCM 
$(10 \mathrm{~mL})$ again. The combined organic layers were washed with saturated brine $(30 \mathrm{~mL} \times 2)$, dried over anhydrous $\mathrm{Na}_{2} \mathrm{SO}_{4}$. After filtration and concentration under reduced pressure, the crude product was purified by column chromatography on silica gel (eluent: petroleum ether/ethyl acetate 30:1) to give $\mathbf{1 h}-d_{1}$ as colorless oil, $428 \mathrm{mg}$, yield $68 \%$; ${ }^{1} \mathrm{H}$ NMR (400 MHz, $\left.\mathrm{CDCl}_{3}\right): \delta 7.32$ (d, $J=8.6 \mathrm{~Hz}, 2 \mathrm{H}), 7.21$ (d, $J=8.6 \mathrm{~Hz}, 2 \mathrm{H}), 5.37$ (s, 1H), 3.85 (s, 3H), 3.59 (s, 3H) ppm.

(b) The reaction of $\mathbf{1} \mathbf{h}-d_{1}$ with Huisgen zwitterion

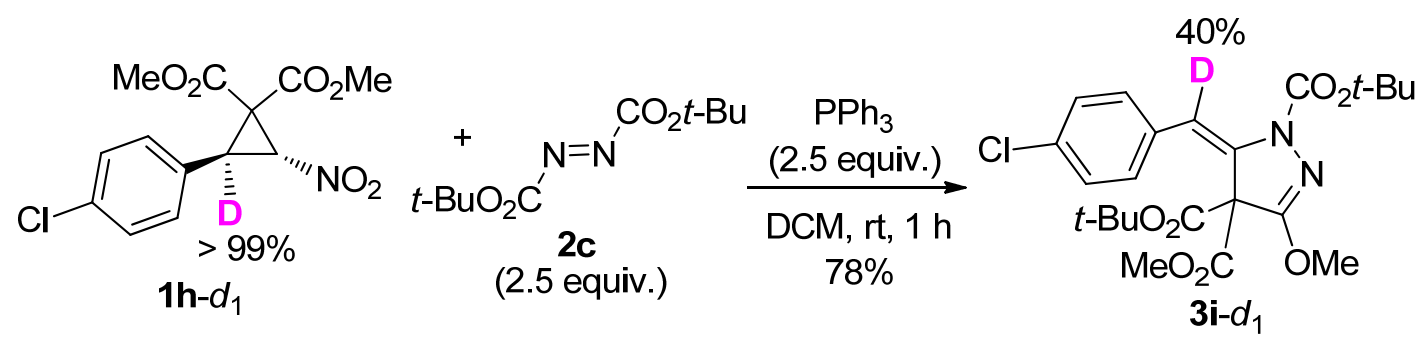

Following the general procedure, the reaction of $\mathbf{1 h}-d_{1}(95 \mathrm{mg}, 0.30 \mathrm{mmol}), \mathbf{2 c}(173 \mathrm{mg}, 0.75$ mmol), and $\mathrm{PPh}_{3}(197 \mathrm{mg}, 0.75 \mathrm{mmol})$ in $\mathrm{CH}_{2} \mathrm{Cl}_{2}(2.0 \mathrm{~mL})$ was performed for $1 \mathrm{~h}$ to afford $3 \mathbf{i}-d_{1}$ as colorless oil, $112 \mathrm{mg}, 78 \%$ yield, $E / Z>20: 1 ;{ }^{1} \mathrm{H}$ NMR (400 MHz, DMSO- $\left.d_{6}\right): \delta 7.44$ (s, 0.6H), 7.37 (br s, 4H), 3.88 (s, 3H), 3.63 (s, 3H), 1.51 (s, 9H), 1.21 (s, 9H) ppm.

(c) ${ }^{1}$ H NMR spectra of the deuterated compounds
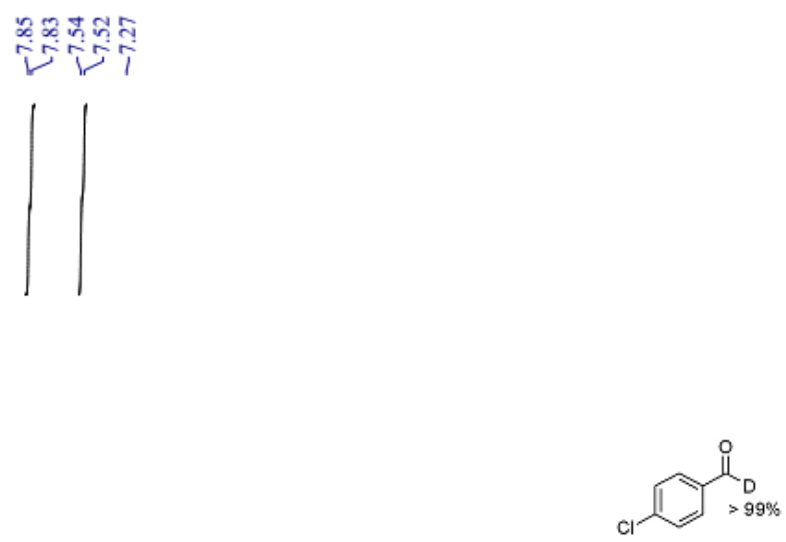

${ }^{1} \mathrm{H}$ NMR $\left(400 \mathrm{MHz}, \mathrm{CDCl}_{3}\right)$

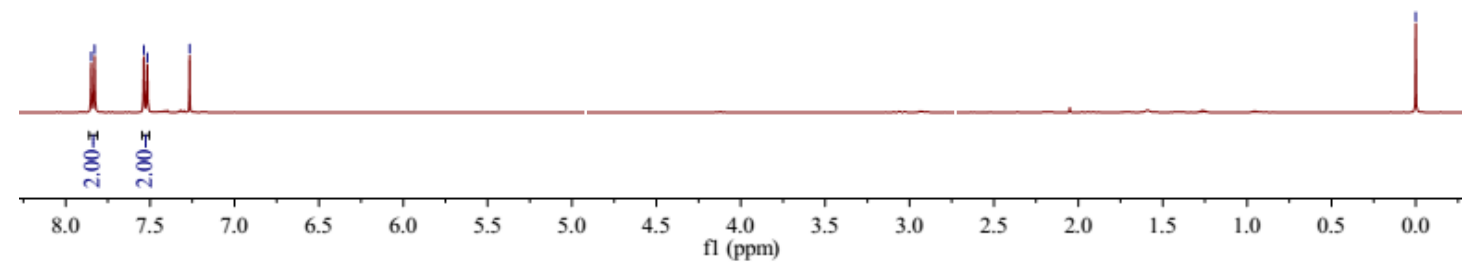




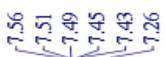

$\frac{5}{1}$

||

5h- $d_{1}$

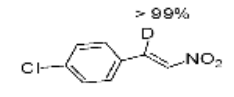

${ }^{1} \mathrm{H}$ NMR (400 MHz, $\mathrm{CDCl}_{3}$ )

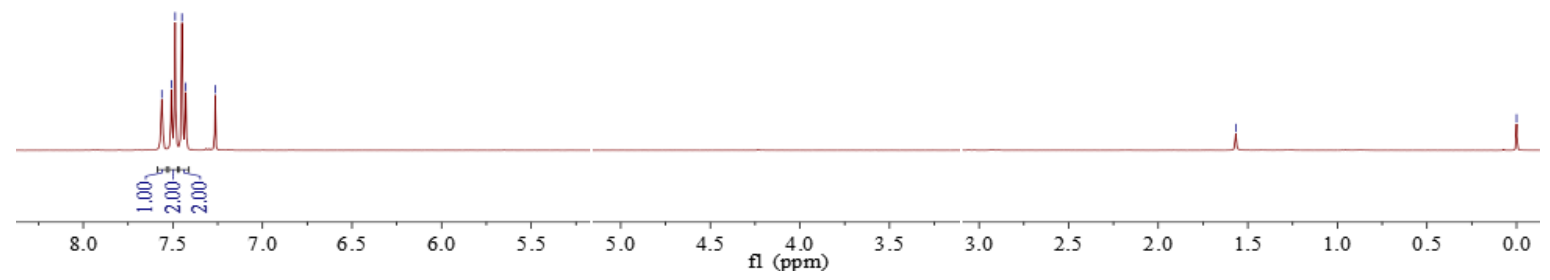

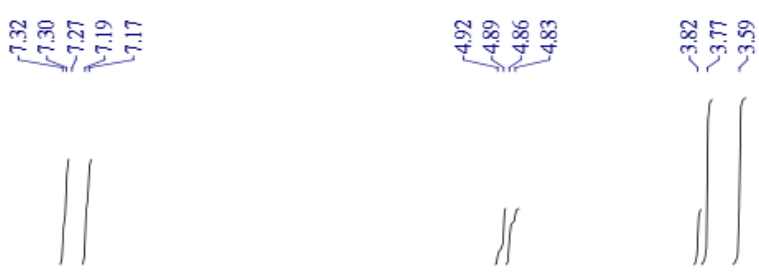
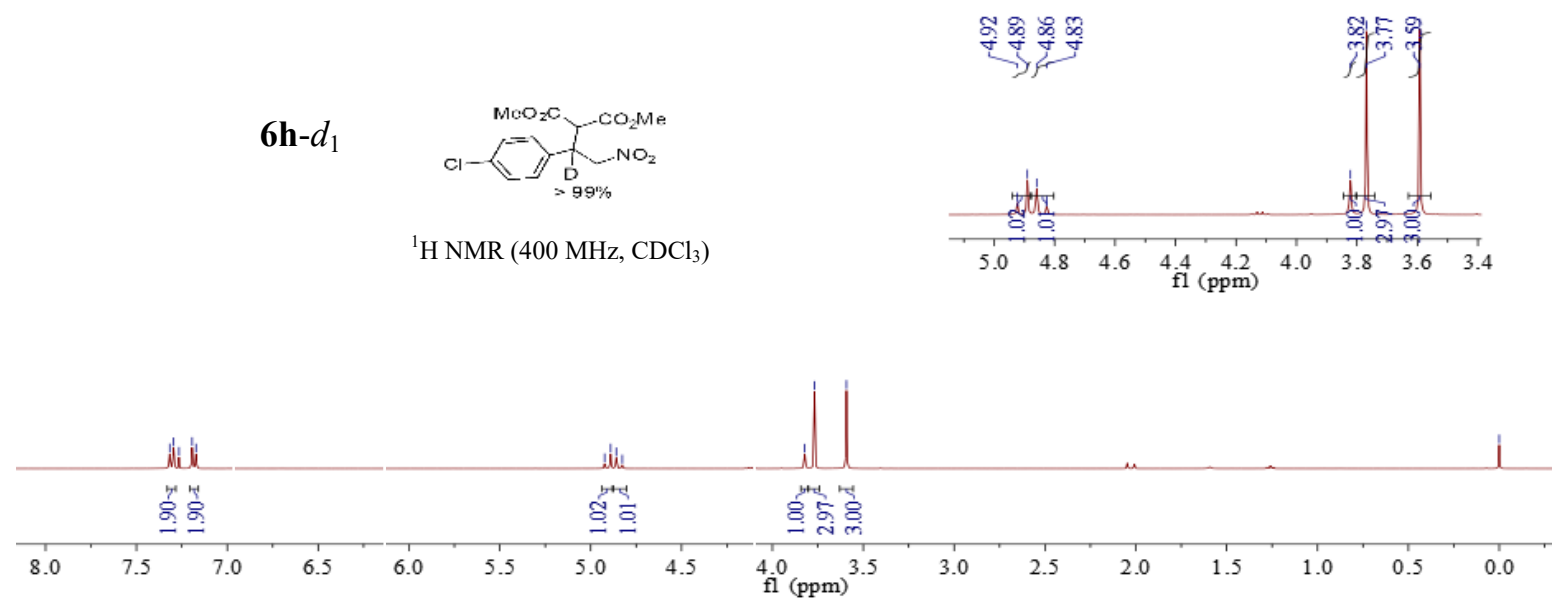


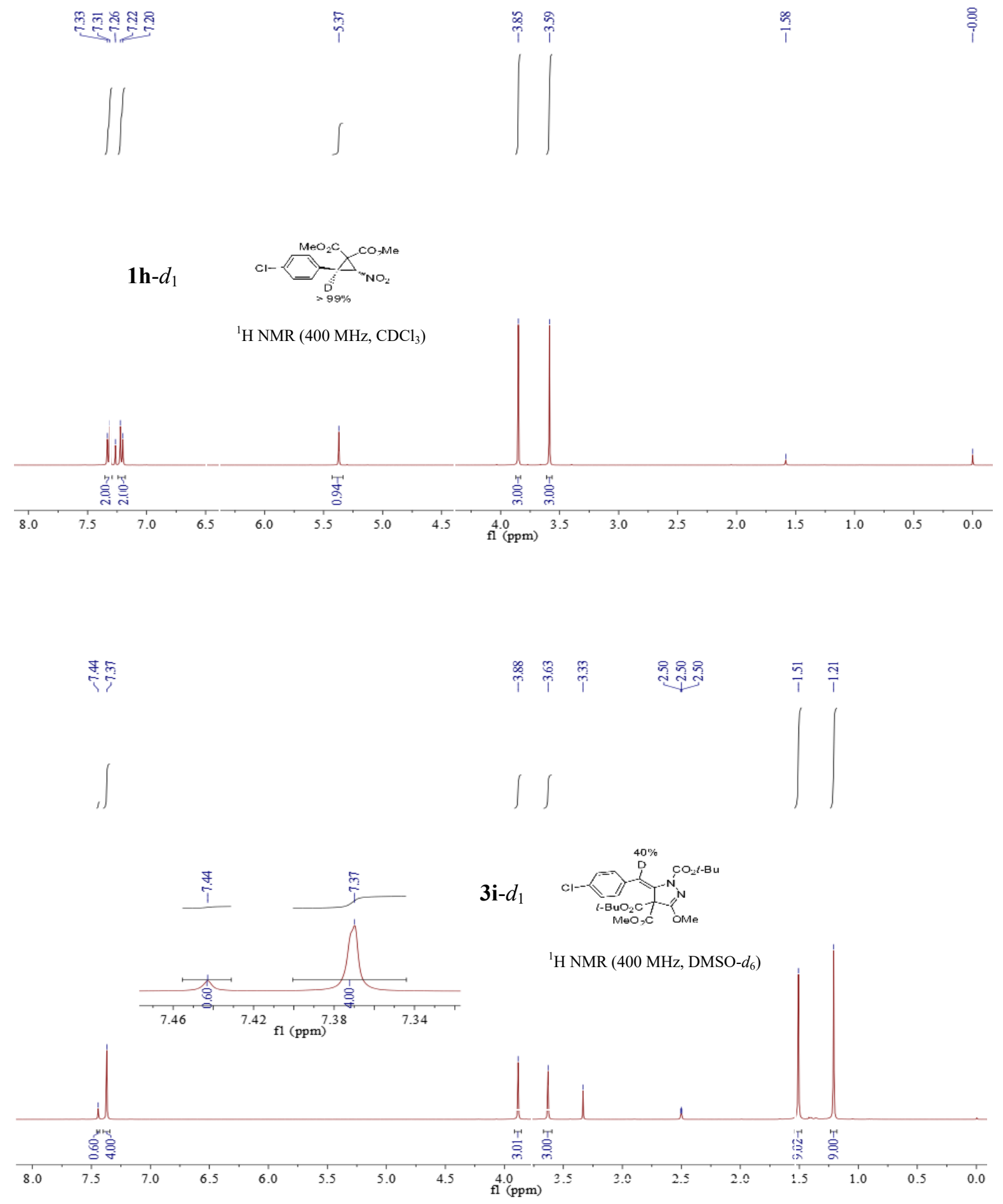




\section{The Reaction of Deuterated Nitrocyclopropane 11- $d_{1}$ with Huisgen Zwitterion}

(a) Preparation of deuterated nitrocyclopropane 11- $d_{1}$<smiles>COC(=O)C1(C(=O)OC)[C@H](c2ccc(Br)cc2)[C@H]1[N+](=O)[O-]</smiles>

To a mixture of $11(358 \mathrm{mg}, 1.0 \mathrm{mmol})$ in $\mathrm{CDCl}_{3}(4.0 \mathrm{~mL})$ and $\mathrm{D}_{2} \mathrm{O}(1.0 \mathrm{~mL})$ was added 1,8-diazabicyclo[5.4.0]undec-7-ene (DBU) $(30 \mu \mathrm{L}, 0.2 \mathrm{mmol})$ by the means of a microsyringe. The resulting mixture was stirred at $\mathrm{rt}$ for $24 \mathrm{~h}$. Then the organic layer was separated and dried over anhydrous $\mathrm{MgSO}_{4}$. After filtration and concentration under reduced pressure, the crude product was purified by column chromatography on silica gel (eluent: petroleum ether/ethyl acetate 30:1) to afford 11- $d_{1}$ as yellow oil (325 mg, yield 91\%). ${ }^{1} \mathrm{H}$ NMR (400 MHz, $\left.\mathrm{CDCl}_{3}\right): \delta$ $7.48(\mathrm{~d}, J=8.4 \mathrm{~Hz}, 2 \mathrm{H}), 7.15(\mathrm{~d}, J=8.4 \mathrm{~Hz}, 2 \mathrm{H}), 5.37$ (d, $J=6.0 \mathrm{~Hz}, 0.18 \mathrm{H}), 4.13$ (br s, 1H), 3.85 (s, 3H), 3.59 (s, 3H) ppm.

(b) The reaction of $11-d_{1}$ with Huisgen zwitterion

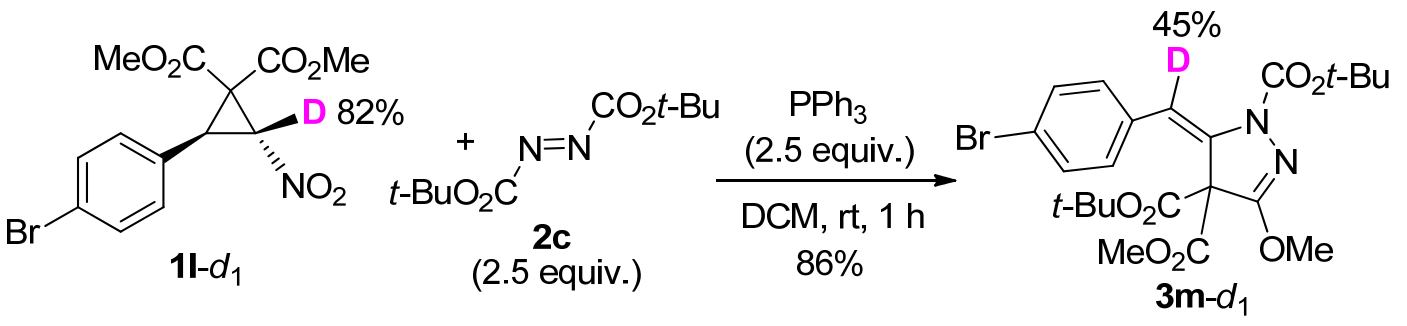

Following the general procedure, the reaction of $11 \mathrm{l}-d_{1}(108 \mathrm{mg}, 0.30 \mathrm{mmol}), \mathbf{2 c}(173 \mathrm{mg}, 0.75$ mmol), and $\mathrm{PPh}_{3}(197 \mathrm{mg}, 0.75 \mathrm{mmol})$ in $\mathrm{CH}_{2} \mathrm{Cl}_{2}(2.0 \mathrm{~mL})$ was performed for $1 \mathrm{~h}$ to afford $\mathbf{3 m}-d_{1}$ as slightly yellow semi-solid, $136 \mathrm{mg}, 86 \%$ yield, $E / Z>20: 1 ;{ }^{1} \mathrm{H}$ NMR (400 MHz, DMSO- $d_{6}$ ): $\delta$ 7.50 (d, $J=8.5 \mathrm{~Hz}, 2 \mathrm{H}), 7.42$ (s, 0.55H), 7.30 (dd, $J=8.5 \mathrm{~Hz}, 2 \mathrm{H}), 3.88$ (s, 3H), 3.63 (s, 3H), 1.51 (s, 9H), $1.21(\mathrm{~s}, 9 \mathrm{H}) \mathrm{ppm}$ 
(c) ${ }^{1} \mathrm{H}$ NMR spectra of the deuterated compounds
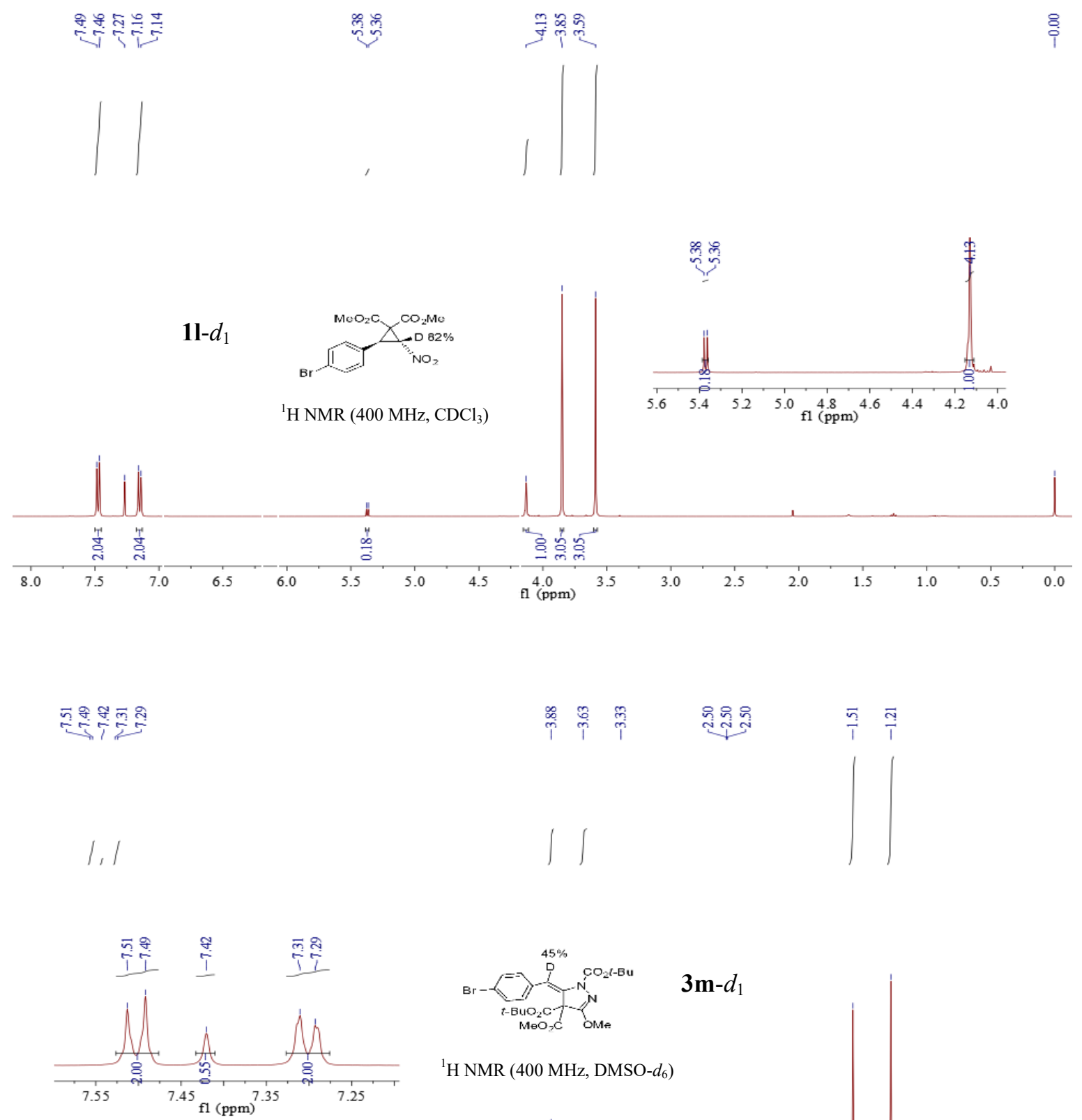

${ }^{1} \mathrm{H}$ NMR (400 MHz, DMSO- $d_{6}$ )

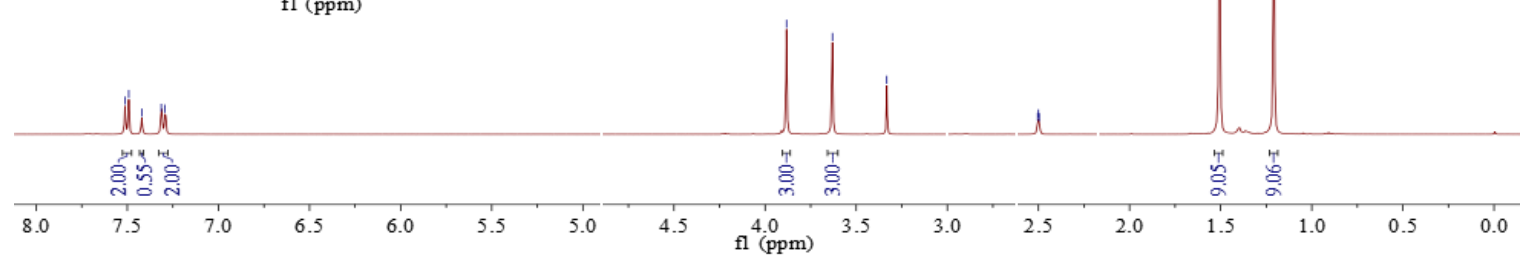

\section{Reference}

5. Allwood, D. M.; Blakemore, D. C.; Ley, S. V. Org. Lett. 2014, 16, 3064-3067. 


\section{Analytical Data for Compounds 3 and 4}<smiles>CCCOC1=NN(C(=O)OCC)C(=Cc2ccccc2)C1(C(=O)OCC)C(=O)OCC</smiles>

Following the general procedure, the reaction of $\mathbf{1 a}(92 \mathrm{mg}, 0.30 \mathrm{mmol}), \mathbf{2 a}(150 \mu \mathrm{L}, 0.75 \mathrm{mmol})$, and $\mathrm{PPh}_{3}(197 \mathrm{mg}, 0.75 \mathrm{mmol})$ in $\mathrm{CH}_{2} \mathrm{Cl}_{2}(2.0 \mathrm{~mL})$ was performed for $48 \mathrm{~h}$ to afford $\mathbf{3 a}, 91 \mathrm{mg}$, $68 \%$ yield, $E / Z>20: 1$; as white solid, mp $91-92{ }^{\circ} \mathrm{C} ;{ }^{1} \mathrm{H}$ NMR (400 MHz, DMSO- $\left.d_{6}\right): \delta 7.54$ (s, 1H), 7.40-7.24 (m, 5H), 5.02-4.94 (m, 1H), 4.85-4.75 (m, 1H), 4.34-4.25 (m, 2H), 4.07-3.99 (m, 2H), 1.29 (d, $J=6.2 \mathrm{~Hz}, 6 \mathrm{H}), 1.23(\mathrm{t}, J=7.1 \mathrm{~Hz}, 3 \mathrm{H}), 1.09$ (d, $J=6.2 \mathrm{~Hz}, 3 \mathrm{H}), 1.02(\mathrm{t}, J=7.1 \mathrm{~Hz}$, $3 \mathrm{H}), 0.93(\mathrm{~d}, J=6.2 \mathrm{~Hz}, 3 \mathrm{H}) \mathrm{ppm} ;{ }^{13} \mathrm{C}$ NMR $\left(100 \mathrm{MHz}\right.$, DMSO- $\left.d_{6}\right): \delta 162.8,162.1,157.4,150.7$, 135.0, 133.7, 128.2, 128.1, 127.5, 116.4, 72.2, 70.7, 69.9, 66.6, 62.7, 21.8, 21.3, 20.9, 20.7, 13.8, 13.4 ppm; HRMS-ESI calcd for $\mathrm{C}_{23} \mathrm{H}_{31} \mathrm{~N}_{2} \mathrm{O}_{7}[\mathrm{M}+\mathrm{H}]^{+} 447.2126$, found 447.2124.<smiles>CCOC(=O)N1N=C(OCC)C(C)(C(=O)OCC)/C1=C\c1ccccc1</smiles>

Following the general procedure, the reaction of $\mathbf{1 a}(92 \mathrm{mg}, 0.30 \mathrm{mmol}), \mathbf{2 b}(119 \mu \mathrm{L}, 0.75 \mathrm{mmol})$, and $\mathrm{PPh}_{3}(197 \mathrm{mg}, 0.75 \mathrm{mmol})$ in $\mathrm{CH}_{2} \mathrm{Cl}_{2}(2.0 \mathrm{~mL})$ was performed for $48 \mathrm{~h}$ to afford $\mathbf{3 b}, 54 \mathrm{mg}$, $43 \%$ yield, $E / Z>20: 1$; as colorless semi-solid; ${ }^{1} \mathrm{H}$ NMR (400 MHz, DMSO- $d_{6}$ ): $\delta 7.57$ (s, $1 \mathrm{H}$ ), 7.34-7.25 (m, 5H), 4.30-4.23 (m, 4H), 4.02 (q, $J=6.8 \mathrm{~Hz}, 4 \mathrm{H}), 1.31-1.24(\mathrm{~m}, 6 \mathrm{H}), 1.01$ (t, $J=$ $6.8 \mathrm{~Hz}, 6 \mathrm{H}) \mathrm{ppm} ;{ }^{13} \mathrm{C}$ NMR (100 MHz, DMSO- $\left.d_{6}\right): \delta 162.6,162.5,157.3,151.0,135.1,133.6$, 128.1, 128.1, 127.5, 116.1, 66.7, 66.6, 62.7, 62.7, 62.0, 14.4, 13.8, 13.4, 13.3 ppm; HRMS-ESI calcd for $\mathrm{C}_{21} \mathrm{H}_{27} \mathrm{~N}_{2} \mathrm{O}_{7}[\mathrm{M}+\mathrm{H}]^{+} 419.1813$, found 419.1811.<smiles>CCOC(=O)N1N=C(OCC)C(C(=O)OCC)(C(=O)OCC)C1=Cc1ccccc1</smiles>

Following the general procedure, the reaction of $1 \mathrm{a}(92 \mathrm{mg}, 0.30 \mathrm{mmol}), \mathbf{2}(173 \mathrm{mg}, 0.75 \mathrm{mmol})$, and $\mathrm{PPh}_{3}(197 \mathrm{mg}, 0.75 \mathrm{mmol})$ in $\mathrm{CH}_{2} \mathrm{Cl}_{2}(2.0 \mathrm{~mL})$ was performed for $12 \mathrm{~h}$ to afford $\mathbf{3 c}, 99 \mathrm{mg}$, $70 \%$ yield, $E / Z>20: 1$; as colorless oil; ${ }^{1} \mathrm{H}$ NMR $\left(400 \mathrm{MHz}\right.$, DMSO- $\left.d_{6}\right): \delta 7.43(\mathrm{~s}, 1 \mathrm{H}), 7.38(\mathrm{~d}, J$ $=7.7 \mathrm{~Hz}, 2 \mathrm{H}), 7.29(\mathrm{t}, J=7.4 \mathrm{~Hz}, 2 \mathrm{H}), 7.25-7.22(\mathrm{~m}, 1 \mathrm{H}), 4.29-4.20(\mathrm{~m}, 2 \mathrm{H}), 4.06-3.96(\mathrm{~m}, 2 \mathrm{H})$, 
$1.50(\mathrm{~s}, 9 \mathrm{H}), 1.27(\mathrm{t}, J=7.0 \mathrm{~Hz}, 3 \mathrm{H}), 1.22(\mathrm{~s}, 9 \mathrm{H}), 1.02(\mathrm{t}, J=7.0 \mathrm{~Hz}, 3 \mathrm{H}) \mathrm{ppm} ;{ }^{13} \mathrm{C}$ NMR $(100$ MHz, DMSO- $\left.d_{6}\right): \delta 163.0,161.4,157.4,150.0,135.3,133.9,128.2,128.1,127.3,116.3,83.7$, 81.6, 67.5, 66.3, 62.5, 27.9, 26.9, 13.8, 13.4 ppm; HRMS-ESI calcd for $\mathrm{C}_{25} \mathrm{H}_{34} \mathrm{~N}_{2} \mathrm{NaO}_{7}[\mathrm{M}+\mathrm{Na}]^{+}$ 497.2258, found 497.2252.

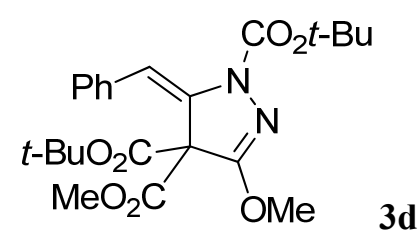

Following the general procedure, the reaction of $\mathbf{1 b}(84 \mathrm{mg}, 0.30 \mathrm{mmol}), \mathbf{2 c}(173 \mathrm{mg}, 0.75 \mathrm{mmol})$, and $\mathrm{PPh}_{3}(197 \mathrm{mg}, 0.75 \mathrm{mmol})$ in $\mathrm{CH}_{2} \mathrm{Cl}_{2}(2.0 \mathrm{~mL})$ was performed for $2 \mathrm{~h}$ to afford $\mathbf{3 d}, 118 \mathrm{mg}$, $88 \%$ yield, $E / Z>20: 1$; as colorless oil; ${ }^{1} \mathrm{H}$ NMR (400 MHz, DMSO- $d_{6}$ ): $\delta 7.47$ (s, $1 \mathrm{H}$ ), 7.35 (d, $J$ $=7.6 \mathrm{~Hz}, 2 \mathrm{H}), 7.30(\mathrm{t}, J=7.1 \mathrm{~Hz}, 2 \mathrm{H}), 7.26-7.22(\mathrm{~m}, 1 \mathrm{H}), 3.88(\mathrm{~s}, 3 \mathrm{H}), 3.59(\mathrm{~s}, 3 \mathrm{H}), 1.51(\mathrm{~s}, 9 \mathrm{H})$, 1.19 (s, 9H) ppm; ${ }^{13} \mathrm{C}$ NMR (100 MHz, DMSO- $\left.d_{6}\right): \delta 163.5,161.2,158.0,149.9,135.5,133.9$, 128.1, 128.1, 127.3, 116.1, 83.9, 81.8, 67.3, 57.6, 53.5, 27.9, 26.9 ppm; HRMS-ESI calcd for $\mathrm{C}_{23} \mathrm{H}_{30} \mathrm{~N}_{2} \mathrm{NaO}_{7}[\mathrm{M}+\mathrm{Na}]^{+}$469.1945, found 469.1951.<smiles>CCCOC(=O)N1N=C(OCC)C(C)(C(=O)OC(C)(C)C)C1=Cc1ccccc1</smiles>

Following the general procedure, the reaction of $1 \mathbf{c}(101 \mathrm{mg}, 0.30 \mathrm{mmol}), \mathbf{2 c}(173 \mathrm{mg}, 0.75 \mathrm{mmol})$, and $\mathrm{PPh}_{3}(197 \mathrm{mg}, 0.75 \mathrm{mmol})$ in $\mathrm{CH}_{2} \mathrm{Cl}_{2}(2.0 \mathrm{~mL})$ was performed for $24 \mathrm{~h}$ to afford $3 \mathrm{e}, 84 \mathrm{mg}$, $56 \%$ yield, $E / Z>20: 1$; as colorless oil; ${ }^{1} \mathrm{H}$ NMR (400 MHz, DMSO- $\left.d_{6}\right): \delta 7.42(\mathrm{~d}, J=7.6 \mathrm{~Hz}, 2 \mathrm{H})$, 7.35 (s, 1H), 7.29 (t, $J=7.5 \mathrm{~Hz}, 2 \mathrm{H}), 7.25-7.20(\mathrm{~m}, 1 \mathrm{H}), 4.93-4.87(\mathrm{~m}, 1 \mathrm{H}), 4.84-4.77(\mathrm{~m}, 1 \mathrm{H})$, 1.49 (s, 9H), 1.29-1.25 (m, 6H), 1.23 (s, 9H), 1.09 (d, $J=6.2 \mathrm{~Hz}, 3 \mathrm{H}), 0.93$ (d, $J=6.2 \mathrm{~Hz}, 3 \mathrm{H})$ ppm; ${ }^{13} \mathrm{C}$ NMR (100 MHz, DMSO- $d_{6}$ ): $\delta$ 162.4, 161.6, 157.0, 150.1, 135.1, 133.9, 128.2, 128.1, 127.3, 116.6, 83.5, 81.4, 73.7, 70.2, 67.5, 27.9, 27.0, 21.0, 20.9, 20.8, 20.7 ppm; HRMS-ESI calcd for $\mathrm{C}_{27} \mathrm{H}_{38} \mathrm{~N}_{2} \mathrm{NaO}_{7}[\mathrm{M}+\mathrm{Na}]^{+}$525.2571, found 525.2574.<smiles>COCC1(C)C(=Cc2ccc(F)cc2)N(C(=O)OC)N=C1OC(=O)OC(C)(C)C</smiles>

Following the general procedure, the reaction of $1 \mathbf{e}(89 \mathrm{mg}, 0.30 \mathrm{mmol}), \mathbf{2 c}(173 \mathrm{mg}, 0.75 \mathrm{mmol})$, 
and $\mathrm{PPh}_{3}(197 \mathrm{mg}, 0.75 \mathrm{mmol})$ in $\mathrm{CH}_{2} \mathrm{Cl}_{2}(2.0 \mathrm{~mL})$ was performed for $1 \mathrm{~h}$ to afford $\mathbf{3 f}, 114 \mathrm{mg}, 82 \%$ yield, $E / Z>20: 1$; as colorless oil; ${ }^{1} \mathrm{H}$ NMR (400 MHz, DMSO- $d_{6}$ ): $\delta 7.44$ (s, 1H), 7.40 (dd, $J=$ 8.5, 5.6 Hz, 2H), 7.14 (t, J=8.9 Hz, 2H), 3.88 (s, 3H), 3.62 (s, 3H), 1.51 (s, 9H), 1.21 (s, 9H) ppm; ${ }^{13} \mathrm{C}$ NMR (100 MHz, DMSO- $\left.d_{6}\right): \delta 163.6,161.4(\mathrm{~d}, J=245.1 \mathrm{~Hz}), 161.2,157.9,149.9,135.5$, $130.3(\mathrm{~d}, J=3.8 \mathrm{~Hz}), 130.2(\mathrm{~d}, J=8.3 \mathrm{~Hz}), 115.1,115.0(\mathrm{~d}, J=21.5 \mathrm{~Hz}), 84.0,81.8,67.3,57.6$, 53.6, 27.8, $26.9 \mathrm{ppm} ;{ }^{19} \mathrm{~F}$ NMR (376 MHz, DMSO- $\left.d_{6}\right): \delta-114.5 \mathrm{ppm}$; HRMS-ESI calcd for $\mathrm{C}_{23} \mathrm{H}_{29} \mathrm{FN}_{2} \mathrm{NaO}_{7}[\mathrm{M}+\mathrm{Na}]^{+}$487.1851, found 487.1849.

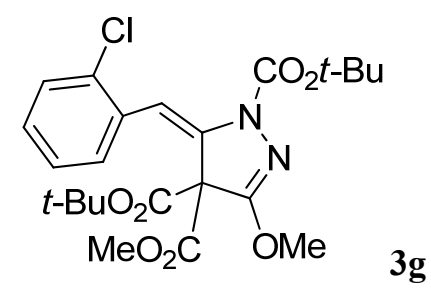

Following the general procedure, the reaction of $\mathbf{1 f}(94 \mathrm{mg}, 0.30 \mathrm{mmol}), \mathbf{2 c}(173 \mathrm{mg}, 0.75 \mathrm{mmol})$, and $\mathrm{PPh}_{3}(197 \mathrm{mg}, 0.75 \mathrm{mmol})$ in $\mathrm{CH}_{2} \mathrm{Cl}_{2}(2.0 \mathrm{~mL})$ was performed for $1 \mathrm{~h}$ to afford $\mathbf{3 g}, 118 \mathrm{mg}$, $82 \%$ yield, $E / Z>20: 1$; as colorless oil; ${ }^{1} \mathrm{H}$ NMR (400 MHz, DMSO- $\left.d_{6}\right): \delta 7.49$ (s, $\left.1 \mathrm{H}\right), 7.49-7.45$ (m, 2H), 7.32-7.24 (m, 2H), $3.88(\mathrm{~s}, 3 \mathrm{H}), 3.61(\mathrm{~s}, 3 \mathrm{H}), 1.52(\mathrm{~s}, 9 \mathrm{H}), 1.21(\mathrm{~s}, 9 \mathrm{H}) \mathrm{ppm} ;{ }^{13} \mathrm{C} \mathrm{NMR}$ (100 MHz, DMSO- $\left.d_{6}\right): \delta 163.3,161.1,157.9,149.8,136.9,132.8,132.5,129.4,129.3,129.0$, 126.9, 112.2, 84.0, 82.1, 67.5, 57.6, 53.6, 27.9, 26.9 ppm; HRMS-ESI calcd for $\mathrm{C}_{23} \mathrm{H}_{29} \mathrm{ClN}_{2} \mathrm{NaO}_{7}$ $[\mathrm{M}+\mathrm{Na}]^{+}$503.1556, found 503.1561.

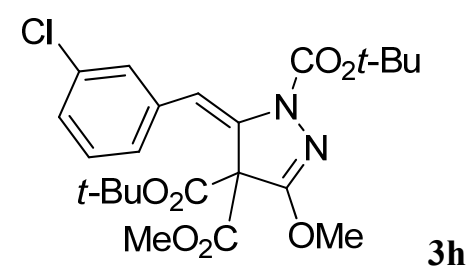

Following the general procedure, the reaction of $1 \mathbf{g}(94 \mathrm{mg}, 0.30 \mathrm{mmol}), \mathbf{2 c}(173 \mathrm{mg}, 0.75 \mathrm{mmol})$, and $\mathrm{PPh}_{3}(197 \mathrm{mg}, 0.75 \mathrm{mmol})$ in $\mathrm{CH}_{2} \mathrm{Cl}_{2}(2.0 \mathrm{~mL})$ was performed for $1 \mathrm{~h}$ to afford $\mathbf{3 h}, 128 \mathrm{mg}$, $89 \%$ yield, $E / Z>20: 1$; as colorless oil; ${ }^{1} \mathrm{H}$ NMR (400 MHz, DMSO- $\left.d_{6}\right): \delta 7.49(\mathrm{~s}, 1 \mathrm{H}), 7.45$ (s, 1H), 7.36-7.26 (m, 3H), 3.89 (s, 3H), 3.62 (s, 3H), 1.51 (s, 9H), 1.23 (s, 9H) ppm; ${ }^{13} \mathrm{C}$ NMR (100 MHz, DMSO- $d_{6}$ ): $\delta 163.5,161.1,157.9,149.8,136.5,136.0,133.0,130.0,127.4,127.1,127.1$, 114.5, 84.2, 82.0, 67.5, 57.6, 53.7, 27.8, 26.9 ppm; HRMS-ESI calcd for $\mathrm{C}_{23} \mathrm{H}_{29} \mathrm{ClN}_{2} \mathrm{NaO}_{7}[\mathrm{M}+$ $\mathrm{Na}]^{+} 503.1556$, found 503.1560 . 


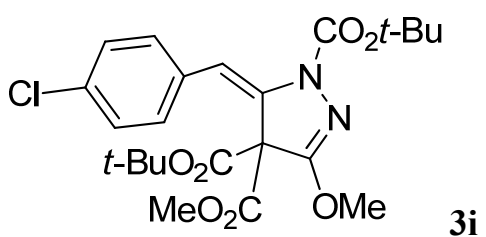

Following the general procedure, the reaction of $\mathbf{1 h}(94 \mathrm{mg}, 0.30 \mathrm{mmol}), \mathbf{2 c}(173 \mathrm{mg}, 0.75 \mathrm{mmol})$, and $\mathrm{PPh}_{3}(197 \mathrm{mg}, 0.75 \mathrm{mmol})$ in $\mathrm{CH}_{2} \mathrm{Cl}_{2}(2.0 \mathrm{~mL})$ was performed for $1 \mathrm{~h}$ to afford $\mathbf{3 i}, 118 \mathrm{mg}, 82 \%$ yield, $E / Z>20: 1$; as colorless oil; ${ }^{1} \mathrm{H}$ NMR (400 MHz, DMSO- $d_{6}$ ): $\delta 7.45$ (s, $1 \mathrm{H}$ ), 7.37 (br s, 4H), $3.88(\mathrm{~s}, 3 \mathrm{H}), 3.63(\mathrm{~s}, 3 \mathrm{H}), 1.51(\mathrm{~s}, 9 \mathrm{H}), 1.21(\mathrm{~s}, 9 \mathrm{H}) \mathrm{ppm} ;{ }^{13} \mathrm{C}$ NMR (100 MHz, DMSO- $\left.d_{6}\right): \delta$ $163.5,161.1,157.9,149.9,136.0,132.8,132.0,129.9,128.2,114.8,84.2,82.0,67.4,57.6,53.7$, 27.8, 26.9 ppm; HRMS-ESI calcd for $\mathrm{C}_{23} \mathrm{H}_{29} \mathrm{ClN}_{2} \mathrm{NaO}_{7}[\mathrm{M}+\mathrm{Na}]^{+}$503.1556, found 503.1555.<smiles>COC(=O)C1(C(=O)OC)C(OC)=NN(C(=O)OC(C)(C)C)/C1=C\c1ccc(Cl)cc1Cl</smiles>

Following the general procedure, the reaction of $1 \mathbf{i}(105 \mathrm{mg}, 0.30 \mathrm{mmol}), \mathbf{2 c}(173 \mathrm{mg}, 0.75 \mathrm{mmol})$, and $\mathrm{PPh}_{3}$ (197 mg, $0.75 \mathrm{mmol}$ ) in $\mathrm{CH}_{2} \mathrm{Cl}_{2}(2.0 \mathrm{~mL})$ was performed for $1 \mathrm{~h}$ to afford $\mathbf{3 j}, 125 \mathrm{mg}, 81 \%$ yield, $E / Z>20: 1$; as colorless oil; ${ }^{1} \mathrm{H}$ NMR (400 MHz, DMSO- $d_{6}$ ): $\delta 7.63(\mathrm{~s}, 1 \mathrm{H}), 7.46(\mathrm{~d}, J=8.4$ Hz, 1H), 7.43 (s, 1H), 7.38 (d, $J=8.4 \mathrm{~Hz}, 1 \mathrm{H}), 3.88$ (s, 3H), 3.65 (s, 3H), 1.51 (s, 9H), $1.24(\mathrm{~s}, 9 \mathrm{H})$ ppm; ${ }^{13} \mathrm{C}$ NMR (100 MHz, DMSO- $\left.d_{6}\right): \delta 163.3,161.1,157.8,149.8,137.4,133.9,133.0,131.7$, 130.6, 128.6, 127.1, 111.0, 84.3, 82.3, 67.6, 57.7, 53.8, 27.8, 26.9 ppm; HRMS-ESI calcd for $\mathrm{C}_{23} \mathrm{H}_{29} \mathrm{Cl}_{2} \mathrm{~N}_{2} \mathrm{O}_{7}[\mathrm{M}+\mathrm{H}]^{+}$515.1346, found 515.1342.

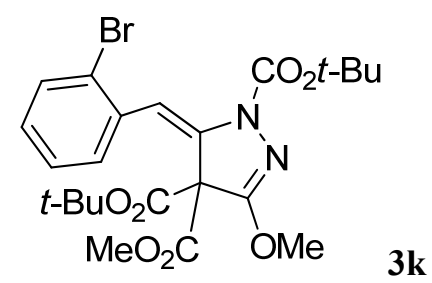

Following the general procedure, the reaction of $\mathbf{1 j}$ (108 $\mathrm{mg}, 0.30 \mathrm{mmol}), \mathbf{2 c}(173 \mathrm{mg}, 0.75 \mathrm{mmol})$, and $\mathrm{PPh}_{3}(197 \mathrm{mg}, 0.75 \mathrm{mmol})$ in $\mathrm{CH}_{2} \mathrm{Cl}_{2}(2.0 \mathrm{~mL})$ was performed for $1 \mathrm{~h}$ to afford $\mathbf{3 k}, 144 \mathrm{mg}$, $91 \%$ yield, $E / Z>20: 1$.

Following the same procedure, a gram-scale synthesis of $\mathbf{3 k}$ was achieved with $\mathbf{1 j}$ (1.07 g, 3.0 mmol), di-tert-butyl azodicarboxylate $2 \mathrm{c}(1.73 \mathrm{~g}, 7.5 \mathrm{mmol})$, and $\mathrm{Ph}_{3} \mathrm{P}(1.97 \mathrm{~g}, 7.5 \mathrm{mmol})$ (reaction time: $1 \mathrm{~h}$ ), $1.37 \mathrm{~g}, 87 \%$ yield, $E / Z>20: 1$. 
Analytical data for 3k: white solid, mp $98-99{ }^{\circ} \mathrm{C} ;{ }^{1} \mathrm{H}$ NMR (400 MHz, DMSO- $\left.d_{6}\right): \delta 7.64$ (d, $J=$ $7.9 \mathrm{~Hz}, 1 \mathrm{H}), 7.48$ (d, $J=7.6 \mathrm{~Hz}, 1 \mathrm{H}), 7.44(\mathrm{~s}, 1 \mathrm{H}), 7.30$ (t, $J=7.6 \mathrm{~Hz}, 1 \mathrm{H}), 7.21$ (t, $J=7.6 \mathrm{~Hz}$, $1 \mathrm{H}), 3.88(\mathrm{~s}, 3 \mathrm{H}), 3.61(\mathrm{~s}, 3 \mathrm{H}), 1.52(\mathrm{~s}, 9 \mathrm{H}), 1.22(\mathrm{~s}, 9 \mathrm{H}) \mathrm{ppm} ;{ }^{13} \mathrm{C}$ NMR $\left(100 \mathrm{MHz}, \mathrm{DMSO}-d_{6}\right): \delta$ 163.3, 161.2, 157.9, 149.8, 136.6, 134.3, 132.2, 129.5, 129.5, 127.5, 123.7, 114.6, 84.0, 82.2, 67.5, 57.6, 53.7, 27.9, 26.9 ppm; HRMS-ESI calcd for $\mathrm{C}_{23} \mathrm{H}_{29} \mathrm{BrN}_{2} \mathrm{NaO}_{7}[\mathrm{M}+\mathrm{Na}]^{+}$547.1050, found 547.1045.

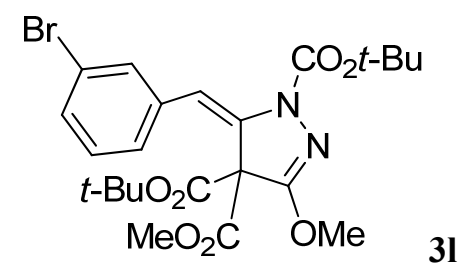

Following the general procedure, the reaction of $\mathbf{1 k}(108 \mathrm{mg}, 0.30 \mathrm{mmol}), \mathbf{2 c}(173 \mathrm{mg}, 0.75$ mmol), and $\mathrm{PPh}_{3}(197 \mathrm{mg}, 0.75 \mathrm{mmol})$ in $\mathrm{CH}_{2} \mathrm{Cl}_{2}(2.0 \mathrm{~mL})$ was performed for $1 \mathrm{~h}$ to afford $3 \mathrm{l}, 150$ $\mathrm{mg}, 95 \%$ yield, $E / Z>20: 1$; as slightly yellow solid, mp 91-92 ${ }^{\circ} \mathrm{C} ;{ }^{1} \mathrm{H}$ NMR $(400 \mathrm{MHz}$, DMSO- $\left.d_{6}\right): \delta 7.62(\mathrm{~s}, 1 \mathrm{H}), 7.46-7.42(\mathrm{~m}, 2 \mathrm{H}), 7.31(\mathrm{~d}, J=7.8 \mathrm{~Hz}, 1 \mathrm{H}), 7.26(\mathrm{t}, J=7.8 \mathrm{~Hz}, 1 \mathrm{H})$, $3.88(\mathrm{~s}, 3 \mathrm{H}), 3.62(\mathrm{~s}, 3 \mathrm{H}), 1.51$ (s, 9H), 1.23 (s, 9H) ppm; ${ }^{13} \mathrm{C}$ NMR (100 MHz, DMSO- $\left.d_{6}\right): \delta$ 163.4, 161.1, 157.9, 149.8, 136.5, 136.3, 130.2, 130.1, 130.0, 127.5, 121.6, 114.5, 84.2, 82.0, 67.5, 57.6, 53.7, 27.8, 26.9 ppm; HRMS-ESI calcd for $\mathrm{C}_{23} \mathrm{H}_{30} \mathrm{BrN}_{2} \mathrm{O}_{7}[\mathrm{M}+\mathrm{H}]^{+}$525.1231, found 525.1237 .

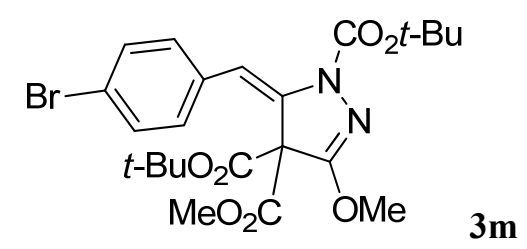

Following the general procedure, the reaction of 11 (108 $\mathrm{mg}, 0.30 \mathrm{mmol}), \mathbf{2}(173 \mathrm{mg}, 0.75 \mathrm{mmol})$, and $\mathrm{PPh}_{3}(197 \mathrm{mg}, 0.75 \mathrm{mmol})$ in $\mathrm{CH}_{2} \mathrm{Cl}_{2}(2.0 \mathrm{~mL})$ was performed for $1 \mathrm{~h}$ to afford $\mathbf{3 m}, 146 \mathrm{mg}$, 93\% yield, $E / Z>20: 1$; as slightly yellow semi-solid; ${ }^{1} \mathrm{H}$ NMR (400 MHz, DMSO- $\left.d_{6}\right): \delta 7.50$ (d, $J$ $=8.5 \mathrm{~Hz}, 2 \mathrm{H}), 7.42(\mathrm{~s}, 1 \mathrm{H}), 7.30(\mathrm{~d}, J=8.5 \mathrm{~Hz}, 2 \mathrm{H}), 3.88(\mathrm{~s}, 3 \mathrm{H}), 3.63(\mathrm{~s}, 3 \mathrm{H}), 1.51(\mathrm{~s}, 9 \mathrm{H}), 1.21$ (s, 9H) ppm; ${ }^{13} \mathrm{C}$ NMR (100 MHz, DMSO- $\left.d_{6}\right): \delta$ 163.4, 161.1, 157.9, 149.9, 136.0, 133.2, 131.1, $130.2,120.6,114.8,84.2,82.0,67.4,57.6,53.7,27.8,26.9$ ppm; HRMS-ESI calcd for $\mathrm{C}_{23} \mathrm{H}_{29} \mathrm{BrN}_{2} \mathrm{NaO}_{7}[\mathrm{M}+\mathrm{Na}]^{+}$547.1050, found 547.1048. 


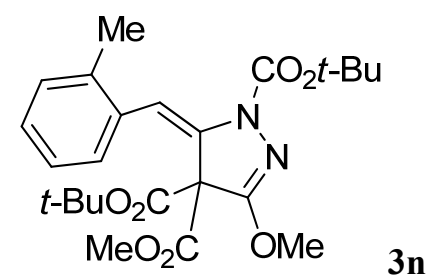

Following the general procedure, the reaction of $1 \mathrm{~m}(88 \mathrm{mg}, 0.30 \mathrm{mmol}), \mathbf{2 c}(173 \mathrm{mg}, 0.75 \mathrm{mmol})$, and $\mathrm{PPh}_{3}(197 \mathrm{mg}, 0.75 \mathrm{mmol})$ in $\mathrm{CH}_{2} \mathrm{Cl}_{2}(2.0 \mathrm{~mL})$ was performed for $2 \mathrm{~h}$ to afford $3 \mathbf{n}, 116 \mathrm{mg}$, 84\% yield, $E / Z>20: 1$; as colorless semi-solid; ${ }^{1} \mathrm{H}$ NMR $\left(400 \mathrm{MHz}, \mathrm{DMSO}-d_{6}\right): \delta 7.38(\mathrm{~s}, 1 \mathrm{H})$, $7.30(\mathrm{~d}, J=7.6 \mathrm{~Hz}, 1 \mathrm{H}), 7.19-7.13(\mathrm{~m}, 2 \mathrm{H}), 7.10-7.06(\mathrm{~m}, 1 \mathrm{H}), 3.86(\mathrm{~s}, 3 \mathrm{H}), 3.51(\mathrm{~s}, 3 \mathrm{H}), 2.16(\mathrm{~s}$, $3 \mathrm{H}), 1.51(\mathrm{~s}, 9 \mathrm{H}), 1.22(\mathrm{~s}, 9 \mathrm{H}) \mathrm{ppm} ;{ }^{13} \mathrm{C}$ NMR (100 MHz, DMSO-d 6 ): $\delta$ 163.5, 161.4, 158.0, $150.0,136.0,135.9,133.3,129.4,127.7,127.6,125.5,114.3,83.6,81.8,67.3,57.5,53.3,27.9$, 26.9, 19.7 ppm; HRMS-ESI calcd for $\mathrm{C}_{24} \mathrm{H}_{32} \mathrm{~N}_{2} \mathrm{NaO}_{7}[\mathrm{M}+\mathrm{Na}]^{+}$483.2102, found 483.2102.

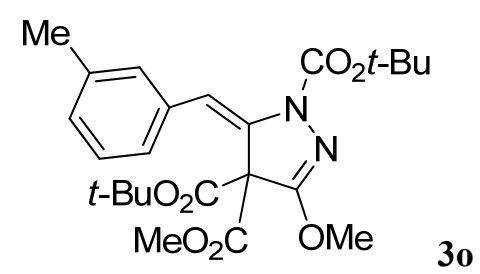

Following the general procedure, the reaction of $1 \mathbf{n}(88 \mathrm{mg}, 0.30 \mathrm{mmol}), \mathbf{2 c}(173 \mathrm{mg}, 0.75 \mathrm{mmol})$, and $\mathrm{PPh}_{3}(197 \mathrm{mg}, 0.75 \mathrm{mmol})$ in $\mathrm{CH}_{2} \mathrm{Cl}_{2}(2.0 \mathrm{~mL})$ was performed for $2 \mathrm{~h}$ to afford $\mathbf{3 o}, 117 \mathrm{mg}$, $85 \%$ yield, $E / Z>20: 1$; as colorless semi-solid; ${ }^{1} \mathrm{H}$ NMR $\left(400 \mathrm{MHz}, \mathrm{DMSO}-d_{6}\right): \delta 7.43(\mathrm{~s}, 1 \mathrm{H})$, $7.20-7.15(\mathrm{~m}, 2 \mathrm{H}), 7.12(\mathrm{~d}, J=7.6 \mathrm{~Hz}, 1 \mathrm{H}), 7.06(\mathrm{~d}, J=7.3 \mathrm{~Hz}, 1 \mathrm{H}), 3.88(\mathrm{~s}, 3 \mathrm{H}), 3.58(\mathrm{~s}, 3 \mathrm{H})$, $2.25(\mathrm{~s}, 3 \mathrm{H}), 1.51(\mathrm{~s}, 9 \mathrm{H}), 1.21(\mathrm{~s}, 9 \mathrm{H}) \mathrm{ppm} ;{ }^{13} \mathrm{C}$ NMR (100 MHz, DMSO-d $): \delta$ 163.5, 161.2, $158.0,149.9,137.1,135.5,133.8,128.6,128.0,127.9,125.3,116.1,83.7,81.7,67.3,57.5,53.4$, 27.9, 26.9, 20.9 ppm; HRMS-ESI calcd for $\mathrm{C}_{24} \mathrm{H}_{33} \mathrm{~N}_{2} \mathrm{O}_{7}[\mathrm{M}+\mathrm{H}]^{+}$461.2282, found 461.2283.

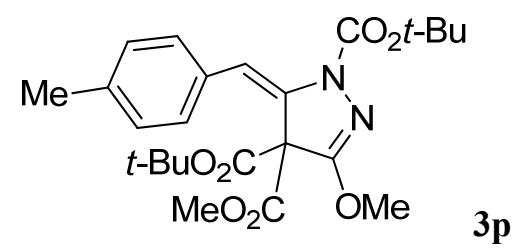

Following the general procedure, the reaction of $10(88 \mathrm{mg}, 0.30 \mathrm{mmol}), \mathbf{2 c}(173 \mathrm{mg}, 0.75 \mathrm{mmol})$, and $\mathrm{PPh}_{3}(197 \mathrm{mg}, 0.75 \mathrm{mmol})$ in $\mathrm{CH}_{2} \mathrm{Cl}_{2}(2.0 \mathrm{~mL})$ was performed for $2 \mathrm{~h}$ to afford $\mathbf{3 p}, 125 \mathrm{mg}$, 91\% yield, $E / Z>20: 1$; as white solid, mp 95-96 ${ }^{\circ} \mathrm{C}$; ${ }^{1} \mathrm{H}$ NMR (400 MHz, DMSO- $d_{6}$ ): $\delta 7.42$ (s, 1H), $7.24(\mathrm{~d}, J=7.9 \mathrm{~Hz}, 2 \mathrm{H}), 7.10$ (d, $J=7.9 \mathrm{~Hz}, 2 \mathrm{H}), 3.88(\mathrm{~s}, 3 \mathrm{H}), 3.59(\mathrm{~s}, 3 \mathrm{H}), 2.27$ (s, 3H), $1.51(\mathrm{~s}, 9 \mathrm{H}), 1.21(\mathrm{~s}, 9 \mathrm{H}) \mathrm{ppm} ;{ }^{13} \mathrm{C} \mathrm{NMR}\left(100 \mathrm{MHz}, \mathrm{DMSO}-d_{6}\right): \delta 163.5,161.3,158.0,150.0$, $136.7,134.9,130.9,128.7,128.1,116.2,83.8,81.7,67.2,57.5,53.5,27.9,26.9,20.7$ ppm; 
HRMS-ESI calcd for $\mathrm{C}_{24} \mathrm{H}_{32} \mathrm{~N}_{2} \mathrm{NaO}_{7}[\mathrm{M}+\mathrm{Na}]^{+}$483.2102, found 483.2105.

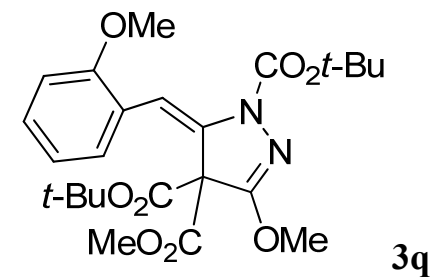

Following the general procedure, the reaction of $1 \mathbf{p}(93 \mathrm{mg}, 0.30 \mathrm{mmol}), \mathbf{2}(173 \mathrm{mg}, 0.75 \mathrm{mmol})$, and $\mathrm{PPh}_{3}(197 \mathrm{mg}, 0.75 \mathrm{mmol})$ in $\mathrm{CH}_{2} \mathrm{Cl}_{2}(2.0 \mathrm{~mL})$ was performed for $2 \mathrm{~h}$ to afford $\mathbf{3 q}, 133 \mathrm{mg}$, 93\% yield, $E / Z>20: 1$; as colorless oil; ${ }^{1} \mathrm{H}$ NMR (400 MHz, DMSO- $\left.d_{6}\right): \delta 7.43(\mathrm{~s}, 1 \mathrm{H}), 7.36(\mathrm{~d}, J$ $=7.4 \mathrm{~Hz}, 1 \mathrm{H}), 7.25(\mathrm{t}, J=7.8 \mathrm{~Hz}, 1 \mathrm{H}), 6.96(\mathrm{~d}, J=8.2 \mathrm{~Hz}, 1 \mathrm{H}), 6.83(\mathrm{t}, J=7.5 \mathrm{~Hz}, 1 \mathrm{H}), 3.87(\mathrm{~s}$, $3 \mathrm{H}), 3.77(\mathrm{~s}, 3 \mathrm{H}), 3.58(\mathrm{~s}, 3 \mathrm{H}), 1.51(\mathrm{~s}, 9 \mathrm{H}), 1.17(\mathrm{~s}, 9 \mathrm{H}) \mathrm{ppm} ;{ }^{13} \mathrm{C}$ NMR $\left(100 \mathrm{MHz}, \mathrm{DMSO}-d_{6}\right): \delta$ 163.7, 161.3, 158.1, 156.5, 149.9, 135.3, 129.1, 128.3, 122.6, 119.8, 112.1, 110.4, 83.6, 81.7, 67.2, 57.5, 55.3, 53.4, 27.9, 26.8 ppm; HRMS-ESI calcd for $\mathrm{C}_{24} \mathrm{H}_{32} \mathrm{~N}_{2} \mathrm{NaO}_{8}[\mathrm{M}+\mathrm{Na}]^{+} 499.2051$, found 499.2058

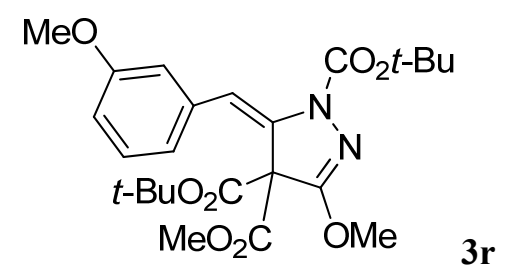

Following the general procedure, the reaction of $1 \mathbf{q}(93 \mathrm{mg}, 0.30 \mathrm{mmol}), \mathbf{2}(173 \mathrm{mg}, 0.75 \mathrm{mmol})$, and $\mathrm{PPh}_{3}(197 \mathrm{mg}, 0.75 \mathrm{mmol})$ in $\mathrm{CH}_{2} \mathrm{Cl}_{2}(2.0 \mathrm{~mL})$ was performed for $2 \mathrm{~h}$ to afford $3 \mathrm{r}, 131 \mathrm{mg}$, 92\% yield, $E / Z>20: 1$; as colorless oil; ${ }^{1} \mathrm{H}$ NMR (400 MHz, DMSO- $\left.d_{6}\right): \delta 7.44(\mathrm{~s}, 1 \mathrm{H}), 7.21(\mathrm{t}, J$ $=7.9 \mathrm{~Hz}, 1 \mathrm{H}), 7.04(\mathrm{br} \mathrm{s}, 1 \mathrm{H}), 6.89(\mathrm{~d}, J=7.7 \mathrm{~Hz}, 1 \mathrm{H}), 6.81(\mathrm{dd}, J=8.2,2.3 \mathrm{~Hz}, 1 \mathrm{H}), 3.88(\mathrm{~s}, 3 \mathrm{H})$, $3.72(\mathrm{~s}, 3 \mathrm{H}), 3.62(\mathrm{~s}, 3 \mathrm{H}), 1.51(\mathrm{~s}, 9 \mathrm{H}), 1.18(\mathrm{~s}, 9 \mathrm{H}) \mathrm{ppm} ;{ }^{13} \mathrm{C}$ NMR (100 MHz, DMSO-d $\left.{ }_{6}\right): \delta$ 163.6, 161.2, 159.1, 158.1, 149.9, 135.6, 135.2, 129.2, 120.8, 116.3, 113.6, 112.6, 83.9, 81.8, 67.3, 57.6, 54.9, 53.6, 27.9, 26.8 ppm; HRMS-ESI calcd for $\mathrm{C}_{24} \mathrm{H}_{33} \mathrm{~N}_{2} \mathrm{O}_{8}[\mathrm{M}+\mathrm{H}]^{+}$477.2231, found 477.2236 .

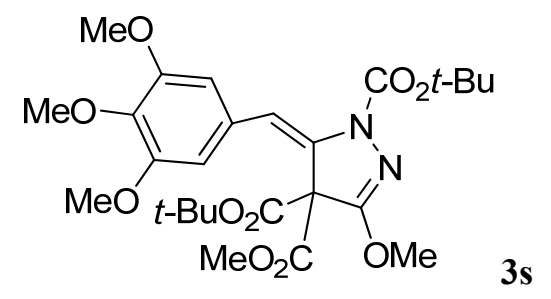

Following the general procedure, the reaction of $1 \mathbf{r}(111 \mathrm{mg}, 0.30 \mathrm{mmol}), 2 \mathbf{c}(173 \mathrm{mg}, 0.75 \mathrm{mmol})$, and $\mathrm{PPh}_{3}(197 \mathrm{mg}, 0.75 \mathrm{mmol})$ in $\mathrm{CH}_{2} \mathrm{Cl}_{2}(2.0 \mathrm{~mL})$ was performed for $2 \mathrm{~h}$ to afford $\mathbf{3 s}, 138 \mathrm{mg}, 86 \%$ 
yield, $E / Z>20: 1$; as slightly yellow oil; ${ }^{1} \mathrm{H}$ NMR (400 MHz, DMSO- $\left.d_{6}\right): \delta 7.40(\mathrm{~s}, 1 \mathrm{H}), 6.78$ (s, $2 \mathrm{H}), 3.89(\mathrm{~s}, 3 \mathrm{H}), 3.74(\mathrm{~s}, 6 \mathrm{H}), 3.67(\mathrm{~s}, 3 \mathrm{H}), 3.62(\mathrm{~s}, 3 \mathrm{H}), 1.51(\mathrm{~s}, 9 \mathrm{H}), 1.15(\mathrm{~s}, 9 \mathrm{H}) \mathrm{ppm} ;{ }^{13} \mathrm{C}$ NMR (100 MHz, DMSO-d $\left.d_{6}\right): \delta 164.0,161.4,158.1,152.6,149.9,136.9,134.8,129.4,117.1$, 105.5, 83.8, 81.8, 67.2, 59.9, 57.6, 56.1, 55.6, 53.8, 27.9, 26.8 ppm; HRMS-ESI calcd for $\mathrm{C}_{26} \mathrm{H}_{40} \mathrm{~N}_{3} \mathrm{O}_{10}\left[\mathrm{M}+\mathrm{NH}_{4}\right]^{+}$554.2708, found 554.2711.

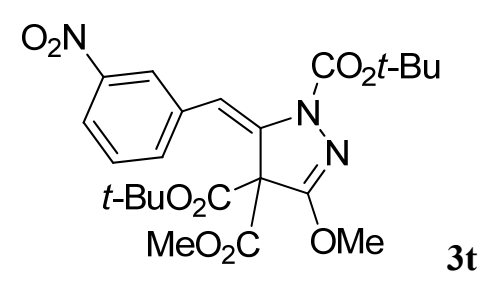

Following the general procedure, the reaction of $1 \mathrm{~s}$ (98 $\mathrm{mg}, 0.30 \mathrm{mmol}), \mathbf{2 c}(173 \mathrm{mg}, 0.75 \mathrm{mmol})$, and $\mathrm{PPh}_{3}(197 \mathrm{mg}, 0.75 \mathrm{mmol})$ in $\mathrm{CH}_{2} \mathrm{Cl}_{2}(2.0 \mathrm{~mL})$ was performed for $1 \mathrm{~h}$ to afford $\mathbf{3 t}, 114 \mathrm{mg}, 78 \%$ yield, $E / Z>20: 1$; as yellow oil; ${ }^{1} \mathrm{H}$ NMR $\left(400 \mathrm{MHz}\right.$, DMSO- $\left.d_{6}\right): \delta 8.29$ (br s, $\left.1 \mathrm{H}\right), 8.12$ (dd, $J=$ 8.1, 1.7 Hz, 1H), $7.76(\mathrm{~d}, J=7.8 \mathrm{~Hz}, 1 \mathrm{H}), 7.62(\mathrm{t}, J=8.0 \mathrm{~Hz}, 1 \mathrm{H}), 7.58(\mathrm{~s}, 1 \mathrm{H}), 3.90(\mathrm{~s}, 3 \mathrm{H}), 3.63$ $(\mathrm{s}, 3 \mathrm{H}), 1.52(\mathrm{~s}, 9 \mathrm{H}), 1.21$ (s, 9H) ppm; ${ }^{13} \mathrm{C}$ NMR (100 MHz, DMSO- $\left.d_{6}\right): \delta$ 163.4, 161.0, 157.8, 149.8, 147.6, 137.4, 135.7, 135.2, 129.7, 122.3, 122.0, 113.5, 84.4, 82.2, 67.7, 57.7, 53.8, 27.8, 26.8 ppm; HRMS-ESI calcd for $\mathrm{C}_{23} \mathrm{H}_{29} \mathrm{~N}_{3} \mathrm{NaO}_{9}[\mathrm{M}+\mathrm{Na}]^{+}$514.1796, found 514.1801.

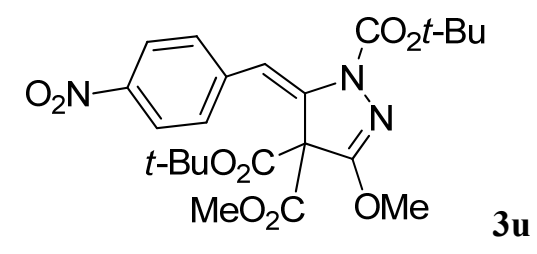

Following the general procedure, the reaction of $\mathbf{1 t}(98 \mathrm{mg}, 0.30 \mathrm{mmol}), \mathbf{2 c}(173 \mathrm{mg}, 0.75 \mathrm{mmol})$, and $\mathrm{PPh}_{3}(197 \mathrm{mg}, 0.75 \mathrm{mmol})$ in $\mathrm{CH}_{2} \mathrm{Cl}_{2}(2.0 \mathrm{~mL})$ was performed for $1 \mathrm{~h}$ to afford $\mathbf{3 u}, 117 \mathrm{mg}$, $80 \%$ yield, $E / Z=5: 1$; as yellow oil; NMR data for the major isomer: ${ }^{1} \mathrm{H}$ NMR $(400 \mathrm{MHz}$, DMSO- $\left.d_{6}\right): \delta 8.17(\mathrm{~d}, J=8.8 \mathrm{~Hz}, 2 \mathrm{H}), 7.62(\mathrm{~s}, 1 \mathrm{H}), 7.61(\mathrm{~d}, J=8.6 \mathrm{~Hz}, 2 \mathrm{H}), 3.90(\mathrm{~s}, 3 \mathrm{H}), 3.65(\mathrm{~s}$, $3 \mathrm{H}), 1.52$ (s, 9H), 1.22 (s, 9H) ppm; ${ }^{13} \mathrm{C}$ NMR (100 MHz, DMSO- $\left.d_{6}\right): \delta 163.2,160.9,158.0$, 155.6, 149.8, 146.1, 138.0, 129.2, 123.3, 113.7, 84.6, 82.4, 67.8, 57.8, 53.9, 27.8, 26.9 ppm; Selected NMR data for the minor isomer: ${ }^{1} \mathrm{H}$ NMR (400 MHz, DMSO- $\left.d_{6}\right): \delta 7.55$ (d, $J=8.8 \mathrm{~Hz}$ ), 3.95 (s), 3.80 (s), 1.41 (s), 1.17 (s) ppm; ${ }^{13} \mathrm{C}$ NMR (100 MHz, DMSO- $\left.d_{6}\right): \delta 162.3,160.7,158.1$, 149.3, 145.5, 141.0, 136.9, 129.6, 123.0, 112.7, 84.3, 82.4, 79.1, 68.9, 57.7, 54.0, 28.1, 27.1 ppm; HRMS-ESI calcd for $\mathrm{C}_{23} \mathrm{H}_{29} \mathrm{~N}_{3} \mathrm{NaO}_{9}[\mathrm{M}+\mathrm{Na}]^{+}$514.1796, found 514.1799. 


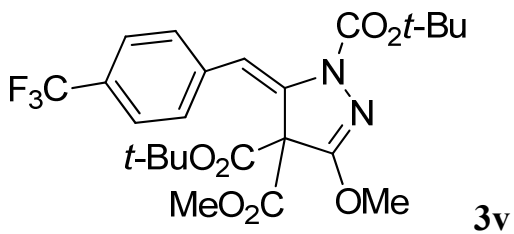

Following the general procedure, the reaction of $\mathbf{1 u}(105 \mathrm{mg}, 0.30 \mathrm{mmol}), \mathbf{2 c}(173 \mathrm{mg}, 0.75$ $\mathrm{mmol}$ ), and $\mathrm{PPh}_{3}(197 \mathrm{mg}, 0.75 \mathrm{mmol})$ in $\mathrm{CH}_{2} \mathrm{Cl}_{2}(2.0 \mathrm{~mL})$ was performed for $1 \mathrm{~h}$ to afford $\mathbf{3 v}$, $128 \mathrm{mg}, 83 \%$ yield, $E / Z>20: 1$; as white solid, mp $79-80{ }^{\circ} \mathrm{C} ;{ }^{1} \mathrm{H}$ NMR (400 MHz, DMSO- $d_{6}$ ): $\delta$ 7.66 (d, $J=8.3 \mathrm{~Hz}, 2 \mathrm{H}), 7.57$ (s, 1H), 7.56 (d, $J=7.8 \mathrm{~Hz}, 2 \mathrm{H}), 3.89$ (s, 3H), 3.64 (s, 3H), 1.52 (s, 9H), 1.18 (s, 9H) ppm; ${ }^{13} \mathrm{C}$ NMR (100 MHz, DMSO- $\left.d_{6}\right): \delta$ 163.4, 161.0, 157.9, 149.8, 138.3, 137.1, 128.8, 127.6 (q, $J=31.8 \mathrm{~Hz}), 125.0$ (q, $J=3.8 \mathrm{~Hz}), 124.2$ (q, $J=271.7 \mathrm{~Hz}), 114.4,84.3$, 82.1, 67.6, 57.7, 53.8, 27.8, 26.8 ppm; ${ }^{19} \mathrm{~F}$ NMR (376 MHz, DMSO- $\left.d_{6}\right): \delta-61.2$ ppm; HRMS-ESI calcd for $\mathrm{C}_{24} \mathrm{H}_{29} \mathrm{~F}_{3} \mathrm{~N}_{2} \mathrm{NaO}_{7}[\mathrm{M}+\mathrm{Na}]^{+}$537.1819, found 537.1822.

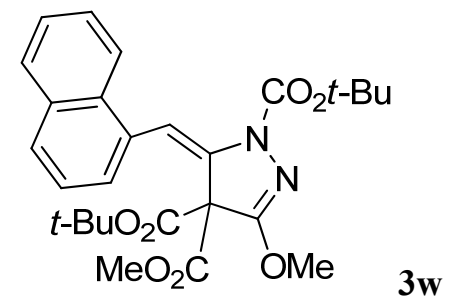

Following the general procedure, the reaction of $1 \mathbf{v}(99 \mathrm{mg}, 0.30 \mathrm{mmol}), \mathbf{2}(173 \mathrm{mg}, 0.75 \mathrm{mmol})$, and $\mathrm{PPh}_{3}(197 \mathrm{mg}, 0.75 \mathrm{mmol})$ in $\mathrm{CH}_{2} \mathrm{Cl}_{2}(2.0 \mathrm{~mL})$ was performed for $1 \mathrm{~h}$ to afford $\mathbf{3 w}, 115 \mathrm{mg}$, $78 \%$ yield, $E / Z>20: 1$; as white solid, mp $156-157{ }^{\circ} \mathrm{C} ;{ }^{1} \mathrm{H}$ NMR (400 MHz, DMSO- $d_{6}$ ): $\delta 7.95-$ $7.91(\mathrm{~m}, 1 \mathrm{H}), 7.87-7.83(\mathrm{~m}, 3 \mathrm{H}), 7.57-7.53(\mathrm{~m}, 2 \mathrm{H}), 7.49$ (d, $J=7.1 \mathrm{~Hz}, 1 \mathrm{H}), 7.43-7.38(\mathrm{~m}, 1 \mathrm{H})$, $3.87(\mathrm{~s}, 3 \mathrm{H}), 3.38(\mathrm{~s}, 3 \mathrm{H}), 1.55$ (s, 9H), $1.00(\mathrm{~s}, 9 \mathrm{H}) \mathrm{ppm} ;{ }^{13} \mathrm{C}$ NMR (100 MHz, DMSO-d $\left.d_{6}\right): \delta$ $163.5,161.3,158.1,150.1,137.2,133.0,131.2,131.2,128.3,127.9,126.3,126.1,125.7,125.3$, 124.4, 112.8, 83.6, 82.0, 67.4, 57.5, 53.2, 27.9, 26.7 ppm; HRMS-ESI calcd for $\mathrm{C}_{27} \mathrm{H}_{32} \mathrm{~N}_{2} \mathrm{NaO}_{7}[\mathrm{M}$ $+\mathrm{Na}]^{+} 519.2102$, found 519.2100 .<smiles>COC(=O)N1N=C(OC(C)(C)C)C(C)(OC(C)(C)C)/C1=C\c1ccco1</smiles>

Following the general procedure, the reaction of $\mathbf{1 w}(81 \mathrm{mg}, 0.30 \mathrm{mmol}), \mathbf{2}(173 \mathrm{mg}, 0.75 \mathrm{mmol})$, and $\mathrm{PPh}_{3}(197 \mathrm{mg}, 0.75 \mathrm{mmol})$ in $\mathrm{CH}_{2} \mathrm{Cl}_{2}(2.0 \mathrm{~mL})$ was performed for $1 \mathrm{~h}$ to afford $\mathbf{3 x}, 85 \mathrm{mg}, 65 \%$ yield, $E / Z>20: 1$; as slightly yellow oil; ${ }^{1} \mathrm{H}$ NMR (400 MHz, DMSO- $\left.d_{6}\right): \delta 7.56$ (br s, $1 \mathrm{H}$ ), 7.43 (s, $1 \mathrm{H}), 6.48-6.45(\mathrm{~m}, 2 \mathrm{H}), 3.89$ (s, 3H), $3.66(\mathrm{~s}, 3 \mathrm{H}), 1.51$ (s, 9H), 1.25 (s, 9H) ppm; ${ }^{13} \mathrm{C}$ NMR (100 
MHz, DMSO- $\left.d_{6}\right): \delta 163.2,161.3,158.0,149.9,149.1,142.5,133.5,111.8,110.7,102.1,83.2$, 82.1, 68.1, 57.7, 53.6, 27.9, 27.0 ppm; HRMS-ESI calcd for $\mathrm{C}_{21} \mathrm{H}_{28} \mathrm{~N}_{2} \mathrm{NaO}_{8}[\mathrm{M}+\mathrm{Na}]^{+} 459.1738$, found 459.1737 .

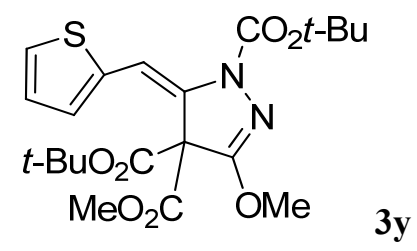

Following the general procedure, the reaction of $1 \mathbf{x}(86 \mathrm{mg}, 0.30 \mathrm{mmol}), \mathbf{2} \mathbf{c}(173 \mathrm{mg}, 0.75 \mathrm{mmol})$, and $\mathrm{PPh}_{3}(197 \mathrm{mg}, 0.75 \mathrm{mmol})$ in $\mathrm{CH}_{2} \mathrm{Cl}_{2}(2.0 \mathrm{~mL})$ was performed for $1 \mathrm{~h}$ to afford $\mathbf{3 y}, 84 \mathrm{mg}, 62 \%$ yield, $E / Z>20: 1$; as slightly red oil; ${ }^{1} \mathrm{H}$ NMR (400 MHz, DMSO- $\left.d_{6}\right): \delta 7.61(\mathrm{~s}, 1 \mathrm{H}), 7.51$ (dd, $J=$ 5.1, $1.1 \mathrm{~Hz}, 1 \mathrm{H}), 7.13(\mathrm{dt}, J=3.7,1.1 \mathrm{~Hz}, 1 \mathrm{H}), 7.02(\mathrm{dd}, J=5.1,3.7 \mathrm{~Hz}, 1 \mathrm{H}), 3.89(\mathrm{~s}, 3 \mathrm{H}), 3.68(\mathrm{~s}$, $3 \mathrm{H}), 1.51(\mathrm{~s}, 9 \mathrm{H}), 1.23$ (s, 9H) ppm; ${ }^{13} \mathrm{C}$ NMR (100 MHz, DMSO-d $\left.d_{6}\right): \delta 163.0,160.7,157.7$, 149.9, 136.4, 134.9, 127.8, 126.8, 126.5, 107.8, 84.0, 82.1, 67.6, 57.7, 53.7, 27.8, 26.9 ppm; HRMS-ESI calcd for $\mathrm{C}_{21} \mathrm{H}_{28} \mathrm{~N}_{2} \mathrm{NaO}_{7} \mathrm{~S}[\mathrm{M}+\mathrm{Na}]^{+}$475.1509, found 475.1512 .

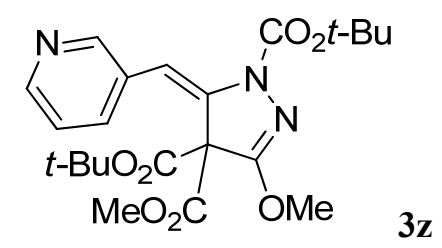

Following the general procedure, the reaction of $1 \mathbf{y}(84 \mathrm{mg}, 0.30 \mathrm{mmol}), \mathbf{2} \mathbf{c}(173 \mathrm{mg}, 0.75 \mathrm{mmol})$, and $\mathrm{PPh}_{3}(197 \mathrm{mg}, 0.75 \mathrm{mmol})$ in $\mathrm{CH}_{2} \mathrm{Cl}_{2}(2.0 \mathrm{~mL})$ was performed for $2 \mathrm{~h}$ to afford $\mathbf{3 z}, 119 \mathrm{mg}, 89 \%$ yield, $E / Z>20: 1$; as colorless oil; ${ }^{1} \mathrm{H}$ NMR $\left(400 \mathrm{MHz}, \mathrm{DMSO}-d_{6}\right): \delta 8.52(\mathrm{~d}, J=1.6 \mathrm{~Hz}, 1 \mathrm{H})$, $8.43(\mathrm{~d}, J=4.8 \mathrm{~Hz}, 1 \mathrm{H}), 7.74(\mathrm{~d}, J=7.9 \mathrm{~Hz}, 1 \mathrm{H}), 7.48(\mathrm{~s}, 1 \mathrm{H}), 7.33(\mathrm{dd}, J=7.9,4.8 \mathrm{~Hz}, 1 \mathrm{H}), 3.89$ $(\mathrm{s}, 3 \mathrm{H}), 3.63(\mathrm{~s}, 3 \mathrm{H}), 1.52(\mathrm{~s}, 9 \mathrm{H}), 1.21(\mathrm{~s}, 9 \mathrm{H}) \mathrm{ppm} ;{ }^{13} \mathrm{C}$ NMR $\left(100 \mathrm{MHz}, \mathrm{DMSO}-d_{6}\right): \delta 163.5$, 161.1, 157.9, 149.9, 149.2, 148.1, 137.1, 135.0, 129.9, 123.1, 112.2, 84.4, 82.2, 67.6, 57.7, 53.8, 27.8, 26.9 ppm; HRMS-ESI calcd for $\mathrm{C}_{22} \mathrm{H}_{30} \mathrm{~N}_{3} \mathrm{O}_{7}[\mathrm{M}+\mathrm{H}]^{+}$448.2078, found 448.2082.<smiles>C=CC=C1N(C(=O)C=Cc2ccccc2)N=C(OC(C)(C)C)C1(C(=O)OC)C(=O)OC</smiles>

Following the general procedure, the reaction of $\mathbf{1 z}(92 \mathrm{mg}, 0.30 \mathrm{mmol}), \mathbf{2 c}(173 \mathrm{mg}, 0.75 \mathrm{mmol})$, and $\mathrm{PPh}_{3}(197 \mathrm{mg}, 0.75 \mathrm{mmol})$ in $\mathrm{CH}_{2} \mathrm{Cl}_{2}(2.0 \mathrm{~mL})$ was performed for $2 \mathrm{~h}$ to afford $\mathbf{3 A}, 119 \mathrm{mg}$, $84 \%$ yield, $E / Z>20: 1$; as yellow oil; ${ }^{1} \mathrm{H}$ NMR $\left(400 \mathrm{MHz}\right.$, DMSO- $\left.d_{6}\right): \delta 7.36-7.32(\mathrm{~m}, 4 \mathrm{H}), 7.25-$ 
$7.20(\mathrm{~m}, 2 \mathrm{H}), 6.90$ (dd, $J=15.3,11.8 \mathrm{~Hz}, 1 \mathrm{H}), 6.65$ (d, $J=15.3 \mathrm{~Hz}, 1 \mathrm{H}), 3.91$ (s, 3H), 3.78 (s, $3 \mathrm{H}), 1.52(\mathrm{~s}, 9 \mathrm{H}), 1.37(\mathrm{~s}, 9 \mathrm{H}) \mathrm{ppm} ;{ }^{13} \mathrm{C}$ NMR (100 MHz, DMSO- $\left.d_{6}\right): \delta 163.9,162.2,158.0$, 149.5, 137.0, 135.0, 132.8, 128.8, 127.7, 125.9, 124.0, 113.8, 84.5, 82.2, 68.1, 57.6, 54.0, 27.8, 27.1 ppm; HRMS-ESI calcd for $\mathrm{C}_{25} \mathrm{H}_{33} \mathrm{~N}_{2} \mathrm{O}_{7}[\mathrm{M}+\mathrm{H}]^{+}$473.2282, found 473.2285.<smiles>CCOC(=O)c1c(OCC)n[nH]c1Cc1ccccc1</smiles>

Following the general procedure, $3 \mathbf{c}(95 \mathrm{mg}, 0.2 \mathrm{mmol})$ was employed to afford $4 \mathbf{a}, 30 \mathrm{mg}, 55 \%$ yield. 4a is a known compound, ${ }^{6}$ white solid, mp $92-93{ }^{\circ} \mathrm{C}$ (ref. $\left.103{ }^{\circ} \mathrm{C}\right) ;{ }^{1} \mathrm{H}$ NMR $(400 \mathrm{MHz}$, DMSO- $d_{6}$ ): $\delta 12.50$ (br s, $\left.1 \mathrm{H}\right), 7.28(\mathrm{t}, J=7.4 \mathrm{~Hz}, 2 \mathrm{H}), 7.24-7.17(\mathrm{~m}, 3 \mathrm{H}), 4.20-4.08(\mathrm{~m}, 6 \mathrm{H})$, 1.29 (t, $J=7.1 \mathrm{~Hz}, 3 \mathrm{H}), 1.16(\mathrm{t}, J=7.1 \mathrm{~Hz}, 3 \mathrm{H}) \mathrm{ppm} ;{ }^{13} \mathrm{C}$ NMR $\left(100 \mathrm{MHz}, \mathrm{DMSO}-d_{6}\right): \delta 162.5$, 162.1, 146.8, 138.2, 128.4, 128.2, 126.4, 95.3, 63.8, 58.8, 31.1, 14.7, 14.2 ppm; HRMS-ESI calcd for $\mathrm{C}_{15} \mathrm{H}_{19} \mathrm{~N}_{2} \mathrm{O}_{3}[\mathrm{M}+\mathrm{H}]^{+}$275.1390, found 275.1396.<smiles>COC(=O)c1c(OC)n[nH]c1Cc1ccccc1</smiles>

Following the general procedure, $\mathbf{3 d}$ ( $90 \mathrm{mg}, 0.2 \mathrm{mmol}$ ) was used to afford $\mathbf{4 b}, 38 \mathrm{mg}$, $78 \%$ yield, white solid, mp $144-145{ }^{\circ} \mathrm{C} ;{ }^{1} \mathrm{H}$ NMR (400 MHz, DMSO- $d_{6}$ ): $\delta 12.58$ (br s, $1 \mathrm{H}$ ), 7.30-7.17 (m, $5 \mathrm{H}), 4.14(\mathrm{~s}, 2 \mathrm{H}), 3.81(\mathrm{~s}, 3 \mathrm{H}), 3.65(\mathrm{~s}, 3 \mathrm{H}) \mathrm{ppm} ;{ }^{13} \mathrm{C}$ NMR (100 MHz, DMSO- $\left.d_{6}\right) \delta 163.1,162.4$, 147.6, 138.1, 128.5, 128.3, 126.4, 94.8, 55.5, 50.5, 31.0 ppm; HRMS-ESI calcd for $\mathrm{C}_{13} \mathrm{H}_{15} \mathrm{~N}_{2} \mathrm{O}_{3}$ $[\mathrm{M}+\mathrm{H}]^{+} 247.1077$, found 247.1081 .

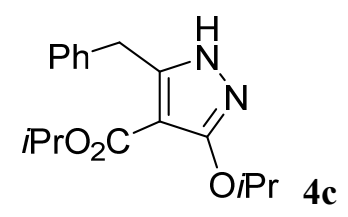

Following the general procedure, $3 \mathbf{e}$ (101 mg, $0.2 \mathrm{mmol}$ ) was employed to afford $4 \mathbf{c}, 41 \mathrm{mg}, 68 \%$ yield, colorless semi-solid; ${ }^{1} \mathrm{H}$ NMR (400 MHz, DMSO- $\left.d_{6}\right)$ : $\delta 12.44$ (br s, $\left.1 \mathrm{H}\right), 7.30-7.26(\mathrm{~m}, 2 \mathrm{H})$, 7.21-7.17 (m, 3H), 4.99-4.89 (m, 1H), 4.84-4.75 (m, 1H), 4.13 (s, 2H), 1.27 (d, $J=6.2 \mathrm{~Hz}, 6 \mathrm{H})$, $1.14(\mathrm{~d}, J=6.2 \mathrm{~Hz}, 6 \mathrm{H}) \mathrm{ppm} ;{ }^{13} \mathrm{C}$ NMR $\left(100 \mathrm{MHz}, \mathrm{DMSO}-d_{6}\right): \delta 161.9,161.7,146.1,138.3$, 128.3, 128.1, 126.3, 96.2, 70.9, 65.9, 31.2, 22.0 (2C), 21.7 (2C) ppm; HRMS-ESI calcd for $\mathrm{C}_{17} \mathrm{H}_{23} \mathrm{~N}_{2} \mathrm{O}_{3}[\mathrm{M}+\mathrm{H}]^{+}$303.1703, found 303.1709. 


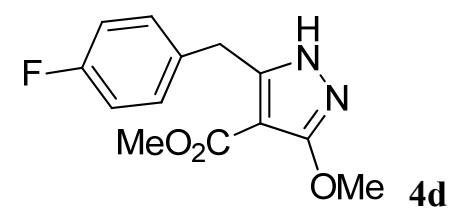

Following the general procedure, $\mathbf{3 f}(93 \mathrm{mg}, 0.2 \mathrm{mmol})$ was employed to afford $\mathbf{4 d}, 38 \mathrm{mg}, 72 \%$ yield, white solid, mp $137-139{ }^{\circ} \mathrm{C} ;{ }^{1} \mathrm{H}$ NMR (400 MHz, DMSO- $d_{6}$ ): $\delta 12.56$ (br s, $\left.1 \mathrm{H}\right), 7.26$ (dd, $J$ $=8.2,5.7 \mathrm{~Hz}, 2 \mathrm{H}), 7.11(\mathrm{t}, J=8.8 \mathrm{~Hz}, 2 \mathrm{H}), 4.12(\mathrm{~s}, 2 \mathrm{H}), 3.81(\mathrm{~s}, 3 \mathrm{H}), 3.66(\mathrm{~s}, 3 \mathrm{H}) \mathrm{ppm} ;{ }^{13} \mathrm{C}$ NMR (100 MHz, DMSO-d $\left.)_{6}\right): \delta 163.0,160.9$ (d, $\left.J=242.0 \mathrm{~Hz}\right), 159.7,147.5,134.2(\mathrm{~d}, J=2.3 \mathrm{~Hz}), 130.2$ $(\mathrm{d}, J=8.2 \mathrm{~Hz}), 115.2(\mathrm{~d}, J=21.3 \mathrm{~Hz}), 94.7,55.5,50.5,30.2 \mathrm{ppm} ;{ }^{19} \mathrm{~F}$ NMR $(376 \mathrm{MHz}$, DMSO-d $)_{6}$ : $\delta$-116.6 ppm; HRMS-ESI calcd for $\mathrm{C}_{13} \mathrm{H}_{14} \mathrm{FN}_{2} \mathrm{O}_{3}[\mathrm{M}+\mathrm{H}]^{+}$265.0983, found 265.0985 .

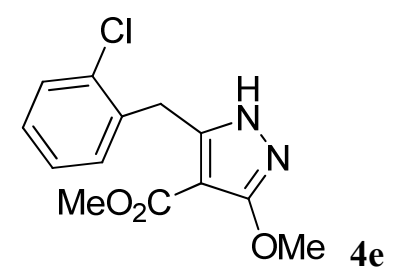

Following the general procedure, $\mathbf{3 g}$ (96 $\mathrm{mg}, 0.2 \mathrm{mmol}$ ) was employed to afford $4 \mathrm{e}, 36 \mathrm{mg}, 65 \%$ yield, white solid, mp $163-164{ }^{\circ} \mathrm{C} ;{ }^{1} \mathrm{H}$ NMR (400 MHz, DMSO- $d_{6}$ ): $\delta 12.47$ (br s, $1 \mathrm{H}$ ), 7.47-7.45 (m, 1H), 7.29-7.24 (m, 2H), 6.98-6.95 (m, 1H), $4.25(\mathrm{~s}, 2 \mathrm{H}), 3.84(\mathrm{~s}, 3 \mathrm{H}), 3.60(\mathrm{~s}, 3 \mathrm{H}) \mathrm{ppm} ;{ }^{13} \mathrm{C}$ NMR (100 MHz, DMSO- $\left.d_{6}\right): \delta 162.9,162.4,145.6,135.2,132.8,129.6,129.2,128.4,127.4,95.6$, 55.6, 50.5, 29.1 ppm; HRMS-ESI calcd for $\mathrm{C}_{13} \mathrm{H}_{14} \mathrm{ClN}_{2} \mathrm{O}_{3}[\mathrm{M}+\mathrm{H}]^{+}$281.0687, found 281.0686.

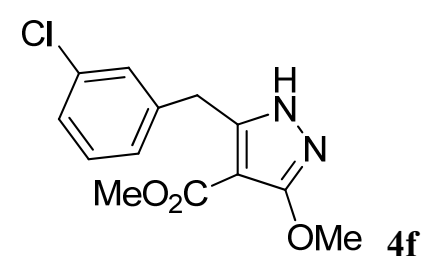

Following the general procedure, $\mathbf{3 h}$ (96 mg, $0.2 \mathrm{mmol}$ ) was employed to afford $\mathbf{4 f}, 37 \mathrm{mg}, 66 \%$ yield, colorless semi-solid; ${ }^{1} \mathrm{H}$ NMR (400 MHz, DMSO- $\left.d_{6}\right)$ : $\delta 12.59$ (br s, $\left.1 \mathrm{H}\right), 7.34-7.26(\mathrm{~m}, 3 \mathrm{H})$, $7.19(\mathrm{~d}, J=7.4 \mathrm{~Hz}, 1 \mathrm{H}), 4.15$ (s, 2H), 3.82 (s, 3H), 3.66 (s, 3H) ppm; ${ }^{13} \mathrm{C}$ NMR (100 MHz, DMSO- $\left.d_{6}\right): \delta 163.0,162.3,146.9,140.5,133.0,130.3,128.2,127.1,126.5,94.9,55.6,50.6,30.6$ ppm; HRMS-ESI calcd for $\mathrm{C}_{13} \mathrm{H}_{14} \mathrm{ClN}_{2} \mathrm{O}_{3}[\mathrm{M}+\mathrm{H}]^{+}$281.0687, found 281.0689.<smiles>COC(=O)c1c(OC)n[nH]c1Cc1ccc(Cl)cc1</smiles> 
Following the general procedure, $\mathbf{3 i}$ (96 $\mathrm{mg}, 0.2 \mathrm{mmol}$ ) was employed to afford $\mathbf{4 g}, 40 \mathrm{mg}, 71 \%$ yield, white solid, mp $147-149{ }^{\circ} \mathrm{C} ;{ }^{1} \mathrm{H}$ NMR (400 MHz, DMSO- $d_{6}$ ): $\delta 12.59$ (br s, $1 \mathrm{H}$ ), 7.35 (d, $J$ $=8.4 \mathrm{~Hz}, 2 \mathrm{H}), 7.24(\mathrm{~d}, J=8.4 \mathrm{~Hz}, 2 \mathrm{H}), 4.12(\mathrm{~s}, 2 \mathrm{H}), 3.81(\mathrm{~s}, 3 \mathrm{H}), 3.65(\mathrm{~s}, 3 \mathrm{H}) \mathrm{ppm} ;{ }^{13} \mathrm{C} \mathrm{NMR}$ (100 MHz, DMSO- $\left.d_{6}\right): \delta 163.0,162.4,147.1,137.0,131.1,130.2,128.4,94.8,55.6,50.5,30.4$ ppm; HRMS-ESI calcd for $\mathrm{C}_{13} \mathrm{H}_{14} \mathrm{ClN}_{2} \mathrm{O}_{3}[\mathrm{M}+\mathrm{H}]^{+}$281.0687, found 281.0692.<smiles>COc1n[nH]c(Cc2ccccc2Br)c1C(C)=O</smiles>

Following the general procedure, 3k (105 mg, $0.2 \mathrm{mmol}$ ) was employed to afford $\mathbf{4 h}, 37 \mathrm{mg}, 57 \%$ yield, white solid, mp $177-178{ }^{\circ} \mathrm{C} ;{ }^{1} \mathrm{H}$ NMR (400 MHz, DMSO- $d_{6}$ ): $\delta 12.46$ (br s, $1 \mathrm{H}$ ), 7.64 (d, $J$ $=7.9 \mathrm{~Hz}, 1 \mathrm{H}), 7.30(\mathrm{t}, J=7.5 \mathrm{~Hz}, 1 \mathrm{H}), 7.19(\mathrm{t}, J=7.6 \mathrm{~Hz}, 1 \mathrm{H}), 6.91(\mathrm{~d}, J=7.6 \mathrm{~Hz}, 1 \mathrm{H}), 4.24(\mathrm{~s}$, $2 \mathrm{H}), 3.85(\mathrm{~s}, 3 \mathrm{H}), 3.61(\mathrm{~s}, 3 \mathrm{H}) \mathrm{ppm} ;{ }^{13} \mathrm{C}$ NMR (100 MHz, DMSO- $\left.d_{6}\right): \delta 162.9,162.4,145.7$, 136.8, 132.5, 129.5, 128.7, 128.0, 123.6, 95.7, 55.6, 50.7, 31.9 ppm; HRMS-ESI calcd for $\mathrm{C}_{13} \mathrm{H}_{14} \mathrm{BrN}_{2} \mathrm{O}_{3}[\mathrm{M}+\mathrm{H}]^{+}$325.0182, found 325.0183.

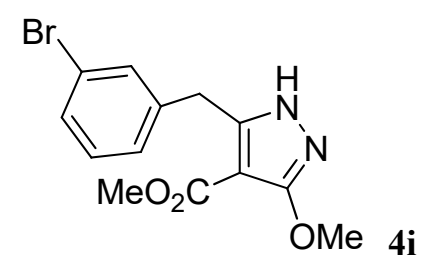

Following the general procedure, 31 (105 mg, $0.2 \mathrm{mmol}$ ) was employed to afford $4 \mathbf{i}, 42 \mathrm{mg}, 65 \%$ yield, colorless semi-solid; ${ }^{1} \mathrm{H}$ NMR (400 MHz, DMSO- $d_{6}$ ): $\delta 12.60$ (br s, $\left.1 \mathrm{H}\right), 7.44$ (s, 1H), 7.41 (d, $J=7.2,1 \mathrm{H}), 7.27-7.22$ (m, 2H), 4.14 (s, 2H), 3.81 (s, 3H), 3.66 (s, 3H) ppm; ${ }^{13} \mathrm{C}$ NMR (100 MHz, DMSO- $\left.d_{6}\right): \delta 163.0,162.4,146.9,140.8,131.1,130.7,129.4,127.5,121.7,94.9,55.6,50.6$, 30.6 ppm; HRMS-ESI calcd for $\mathrm{C}_{13} \mathrm{H}_{14} \mathrm{BrN}_{2} \mathrm{O}_{3}[\mathrm{M}+\mathrm{H}]^{+}$325.0182, found 325.0182.

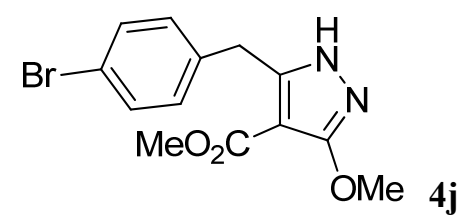

Following the general procedure, $\mathbf{3 m}$ (105 mg, $0.2 \mathrm{mmol}$ ) was employed to afford $\mathbf{4 j}$, $43 \mathrm{mg}, 66 \%$ yield, white solid, mp $150-151{ }^{\circ} \mathrm{C} ;{ }^{1} \mathrm{H}$ NMR (400 MHz, DMSO- $d_{6}$ ): $\delta 12.57$ (br s, $1 \mathrm{H}$ ), 7.48 (d, $J$ $=8.3 \mathrm{~Hz}, 2 \mathrm{H}), 7.18(\mathrm{~d}, J=8.3 \mathrm{~Hz}, 2 \mathrm{H}), 4.11(\mathrm{~s}, 2 \mathrm{H}), 3.82(\mathrm{~s}, 3 \mathrm{H}), 3.65(\mathrm{~s}, 3 \mathrm{H}) \mathrm{ppm} ;{ }^{13} \mathrm{C} \mathrm{NMR}$ (100 MHz, DMSO- $\left.d_{6}\right): \delta 163.0,162.4,147.0,137.4,131.3,130.5,119.6,94.9,55.5,50.5,30.5$ 
ppm; HRMS-ESI calcd for $\mathrm{C}_{13} \mathrm{H}_{14} \mathrm{BrN}_{2} \mathrm{O}_{3}[\mathrm{M}+\mathrm{H}]^{+}$325.0182, found 325.0185.

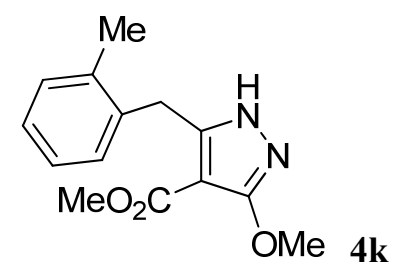

Following the general procedure, $\mathbf{3 n}$ ( $92 \mathrm{mg}, 0.2 \mathrm{mmol}$ ) was employed to afford $\mathbf{4 k}, 30 \mathrm{mg}, 58 \%$ yield, white solid, mp $169-170{ }^{\circ} \mathrm{C} ;{ }^{1} \mathrm{H}$ NMR (400 MHz, DMSO- $d_{6}$ ): $\delta 12.39$ (br s, $1 \mathrm{H}$ ), 7.17 (d, $J$ $=8.5 \mathrm{~Hz}, 1 \mathrm{H}), 7.14-7.07(\mathrm{~m}, 2 \mathrm{H}), 6.82(\mathrm{~d}, J=8.5 \mathrm{~Hz}, 1 \mathrm{H}), 4.13(\mathrm{~s}, 2 \mathrm{H}), 3.83(\mathrm{~s}, 3 \mathrm{H}), 3.61(\mathrm{~s}, 3 \mathrm{H})$, $2.28(\mathrm{~s}, 3 \mathrm{H}) \mathrm{ppm} ;{ }^{13} \mathrm{C}$ NMR (100 MHz, DMSO- $\left.d_{6}\right): \delta 163.1,162.3,147.0,136.1,135.7,129.9$, 127.8, 126.4, 126.0, 95.3, 55.5, 50.5, 28.5, $19.2 \mathrm{ppm}$; HRMS-ESI calcd for $\mathrm{C}_{14} \mathrm{H}_{17} \mathrm{~N}_{2} \mathrm{O}_{3}[\mathrm{M}+\mathrm{H}]^{+}$ 261.1234, found 261.1232.

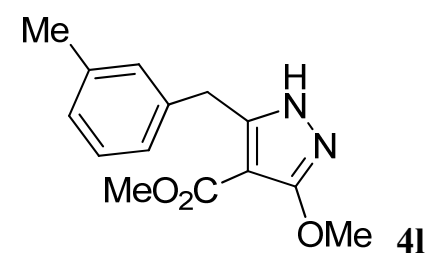

Following the general procedure, 30 (92 $\mathrm{mg}, 0.2 \mathrm{mmol}$ ) was employed to afford 4l, $33 \mathrm{mg}, 64 \%$ yield, colorless semi-solid; ${ }^{1} \mathrm{H}$ NMR (400 MHz, DMSO- $d_{6}$ ): $\delta 12.54$ (br s, $1 \mathrm{H}$ ), 7.16 (t, $J=7.5 \mathrm{~Hz}$, 1H), 7.04-6.99 (m, 3H), 4.10 (s, 2H), $3.80(\mathrm{~s}, 3 \mathrm{H}), 3.65$ (s, 3H), $3.36(\mathrm{~s}, 3 \mathrm{H}) \mathrm{ppm} ;{ }^{13} \mathrm{C}$ NMR (100 MHz, DMSO- $\left.d_{6}\right): \delta 163.1,162.3,147.6,138.0,137.5,128.9,128.4,127.1,125.4,94.8,55.5,50.5$, 30.9, 21.0 ppm; HRMS-ESI calcd for $\mathrm{C}_{14} \mathrm{H}_{17} \mathrm{~N}_{2} \mathrm{O}_{3}[\mathrm{M}+\mathrm{H}]^{+}$261.1234, found 261.1236.<smiles>COC(=O)c1c(OC)n[nH]c1Cc1ccc(C)cc1</smiles>

Following the general procedure, $\mathbf{3 p}$ ( $92 \mathrm{mg}, 0.2 \mathrm{mmol}$ ) was employed to afford $\mathbf{4 m}, 35 \mathrm{mg}, 68 \%$ yield, white solid, mp $132-133{ }^{\circ} \mathrm{C} ;{ }^{1} \mathrm{H}$ NMR (400 MHz, DMSO- $\left.d_{6}\right): \delta 12.52$ (br s, $1 \mathrm{H}$ ), 7.11 (d, $J$ $=8.3 \mathrm{~Hz}, 2 \mathrm{H}), 7.08(\mathrm{~d}, J=8.3 \mathrm{~Hz}, 2 \mathrm{H}), 4.08(\mathrm{~s}, 2 \mathrm{H}), 3.80(\mathrm{~s}, 3 \mathrm{H}), 3.65(\mathrm{~s}, 3 \mathrm{H}), 2.24(\mathrm{~s}, 3 \mathrm{H}) \mathrm{ppm}$; ${ }^{13} \mathrm{C}$ NMR (100 MHz, DMSO- $\left.d_{6}\right): \delta 163.0,162.3,147.8,135.4,135.0,129.0,128.2,94.7,55.5$, 50.4, 30.6, 20.6 ppm; HRMS-ESI calcd for $\mathrm{C}_{14} \mathrm{H}_{17} \mathrm{~N}_{2} \mathrm{O}_{3}[\mathrm{M}+\mathrm{H}]^{+}$261.1234, found 261.1238. 


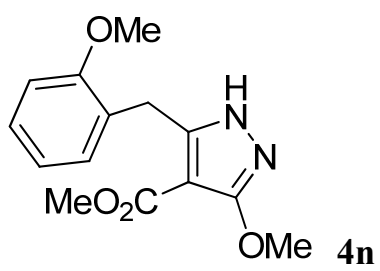

Following the general procedure, 3q (96 mg, $0.2 \mathrm{mmol}$ ) was employed to afford $\mathbf{4 n}, 32 \mathrm{mg}, 58 \%$ yield, white solid, mp $154-156{ }^{\circ} \mathrm{C} ;{ }^{1} \mathrm{H}$ NMR (400 MHz, DMSO- $d_{6}$ ): $\delta 12.33$ (br s, $1 \mathrm{H}$ ), 7.20 (t, $J$ $=7.0 \mathrm{~Hz}, 1 \mathrm{H}), 6.96(\mathrm{~d}, J=8.2 \mathrm{~Hz}, 1 \mathrm{H}), 6.90-6.82(\mathrm{~m}, 2 \mathrm{H}), 4.11(\mathrm{~s}, 2 \mathrm{H}), 3.83(\mathrm{~s}, 3 \mathrm{H}), 3.80(\mathrm{~s}, 3 \mathrm{H})$, $3.63(\mathrm{~s}, 3 \mathrm{H}) \mathrm{ppm} ;{ }^{13} \mathrm{C}$ NMR (100 MHz, DMSO- $\left.d_{6}\right): \delta 163.1,162.3,156.8,146.8,128.8,127.9$, 125.6, 120.3, 110.5, 95.3, 55.5, 55.3, 50.4, 25.7 ppm; HRMS-ESI calcd for $\mathrm{C}_{14} \mathrm{H}_{17} \mathrm{~N}_{2} \mathrm{O}_{4}[\mathrm{M}+\mathrm{H}]^{+}$ 277.1183, found 277.1188.<smiles>COc1cccc(Cc2[nH]nc(OC)c2C(C)=O)c1</smiles>

Following the general procedure, $3 \mathbf{r}(96 \mathrm{mg}, 0.2 \mathrm{mmol}$ ) was employed to afford $4 \mathbf{4 0}, 33 \mathrm{mg}, 60 \%$ yield, colorless semi-solid; ${ }^{1} \mathrm{H}$ NMR (400 MHz, DMSO- $\left.d_{6}\right): \delta 12.55$ (br s, $\left.1 \mathrm{H}\right), 7.19$ (t, $J=7.9 \mathrm{~Hz}$, $1 \mathrm{H}), 6.83-6.76(\mathrm{~m}, 3 \mathrm{H}), 4.11(\mathrm{~s}, 2 \mathrm{H}), 3.81(\mathrm{~s}, 3 \mathrm{H}), 3.71(\mathrm{~s}, 3 \mathrm{H}), 3.67$ (s, 3H) ppm; ${ }^{13} \mathrm{C}$ NMR $(100$ MHz, DMSO- $\left.d_{6}\right): \delta 163.1,162.4,159.3,147.5,139.6,129.5,120.5,114.3,111.7,94.8,55.5,54.9$, 50.5, 31.0 ppm; HRMS-ESI calcd for $\mathrm{C}_{14} \mathrm{H}_{17} \mathrm{~N}_{2} \mathrm{O}_{4}[\mathrm{M}+\mathrm{H}]^{+}$277.1183, found 277.1186.

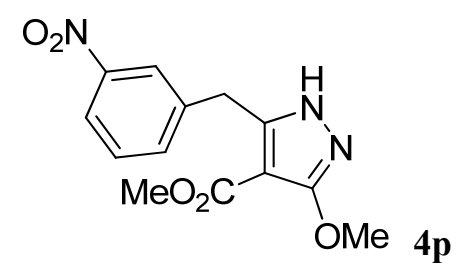

Following the general procedure, $3 \mathbf{t}$ ( $99 \mathrm{mg}, 0.2 \mathrm{mmol}$ ) was employed to afford $\mathbf{4 p}, 30 \mathrm{mg}, 52 \%$ yield, white solid, mp $176-177{ }^{\circ} \mathrm{C} ;{ }^{1} \mathrm{H}$ NMR (400 MHz, DMSO- $d_{6}$ ): $\delta 12.69$ (br s, 1H), 8.12 (s, 1H), 8.09 (d, $J=8.1 \mathrm{~Hz}, 1 \mathrm{H}), 7.70$ (d, $J=7.8 \mathrm{~Hz}, 1 \mathrm{H}), 7.61$ (t, $J=7.9 \mathrm{~Hz}, 1 \mathrm{H}), 4.28$ (s, 2H), 3.82 (s, 3H), 3.66 (s, 3H) ppm; ${ }^{13} \mathrm{C}$ NMR (100 MHz, DMSO- $\left.d_{6}\right): \delta$ 163.0, 162.4, 147.8, 146.5, 140.2, 135.3, 130.1, 123.1, 121.7, 95.0, 55.6, 50.7, 30.7 ppm; HRMS-ESI calcd for $\mathrm{C}_{13} \mathrm{H}_{14} \mathrm{~N}_{3} \mathrm{O}_{5}[\mathrm{M}+\mathrm{H}]^{+}$ 292.0928, found 292.0932 .<smiles>COC(=O)c1c(OC)n[nH]c1Cc1ccc([N+](=O)[O-])cc1</smiles> 
Following the general procedure, $3 \mathbf{u}(99 \mathrm{mg}, 0.2 \mathrm{mmol})$ was employed to afford $\mathbf{4 q}, 31 \mathrm{mg}, 54 \%$ yield, white solid, mp $196-197{ }^{\circ} \mathrm{C} ;{ }^{1} \mathrm{H}$ NMR (400 MHz, DMSO- $d_{6}$ ): $\delta 12.67$ (br s, $1 \mathrm{H}$ ), 8.16 (d, $J$ $=8.0 \mathrm{~Hz}, 2 \mathrm{H}), 7.47(\mathrm{~d}, J=8.0 \mathrm{~Hz}, 2 \mathrm{H}), 4.28(\mathrm{~s}, 2 \mathrm{H}), 3.82(\mathrm{~s}, 3 \mathrm{H}), 3.64(\mathrm{~s}, 3 \mathrm{H}) \mathrm{ppm} ;{ }^{13} \mathrm{C} \mathrm{NMR}$ (100 MHz, DMSO- $\left.d_{6}\right): \delta 162.9,162.4,146.3,146.1,145.9,129.5,123.7,95.2,55.6,50.6,31.0$ ppm; HRMS-ESI calcd for $\mathrm{C}_{13} \mathrm{H}_{14} \mathrm{~N}_{3} \mathrm{O}_{5}[\mathrm{M}+\mathrm{H}]^{+} 292.0928$, found 292.0933 .<smiles>COC(=O)c1c(OC)n[nH]c1Cc1ccc(C(F)(F)F)cc1</smiles>

Following the general procedure, $3 \mathbf{v}$ (103 mg, $0.2 \mathrm{mmol}$ ) was employed to afford $4 \mathbf{r}, 40 \mathrm{mg}, 64 \%$ yield, white solid, mp $183-184{ }^{\circ} \mathrm{C} ;{ }^{1} \mathrm{H}$ NMR (400 MHz, DMSO- $\left.d_{6}\right): \delta 12.64$ (br s, $1 \mathrm{H}$ ), 7.66 (d, $J=$ $8.0 \mathrm{~Hz}, 2 \mathrm{H}), 7.43(\mathrm{~d}, J=8.0 \mathrm{~Hz}, 2 \mathrm{H}), 4.24(\mathrm{~s}, 2 \mathrm{H}), 3.82(\mathrm{~s}, 3 \mathrm{H}), 3.65$ (s, 3H) ppm; ${ }^{13} \mathrm{C} \mathrm{NMR}(100 \mathrm{MHz}$, DMSO- $\left.d_{6}\right): \delta$ 163.0, 162.4, 146.6, 142.8, 129.1, 127.2 (q, $\left.J=31.6 \mathrm{~Hz}\right), 125.3$ (q, $\left.J=3.6 \mathrm{~Hz}\right), 124.3(\mathrm{q}$, $J=271.7 \mathrm{~Hz}), 95.0,55.6,50.5,30.9 \mathrm{ppm} ;{ }^{19} \mathrm{~F}$ NMR (376 MHz, DMSO- $\left.d_{6}\right): \delta-60.9 \mathrm{ppm}$; HRMS-ESI calcd for $\mathrm{C}_{14} \mathrm{H}_{14} \mathrm{~F}_{3} \mathrm{~N}_{2} \mathrm{O}_{3}[\mathrm{M}+\mathrm{H}]^{+}$315.0951, found 315.0955.<smiles>COC(=O)c1c(OC)n[nH]c1Cc1ccc(Cl)cc1Cl</smiles>

Following the general procedure, $\mathbf{3 j}$ (103 $\mathrm{mg}, 0.2 \mathrm{mmol}$ ) was employed to afford $\mathbf{4 s}, 38 \mathrm{mg}, 61 \%$ yield, white solid, mp $149-151{ }^{\circ} \mathrm{C} ;{ }^{1} \mathrm{H}$ NMR (400 MHz, DMSO- $\left.d_{6}\right): \delta 12.47$ (br s, $1 \mathrm{H}$ ), 7.63 (d, $J$ $=2.1 \mathrm{~Hz}, 1 \mathrm{H}), 7.36(\mathrm{dd}, J=8.4,2.1 \mathrm{~Hz}, 1 \mathrm{H}), 7.02(\mathrm{~d}, J=8.4 \mathrm{~Hz}, 1 \mathrm{H}), 4.22(\mathrm{~s}, 2 \mathrm{H}), 3.84(\mathrm{~s}, 3 \mathrm{H})$, $3.61(\mathrm{~s}, 3 \mathrm{H}) \mathrm{ppm} ;{ }^{13} \mathrm{C}$ NMR (100 MHz, DMSO-d $\left.{ }_{6}\right): \delta 162.8,162.4,145.3,134.2,133.8,132.1$, 131.2, 128.7, 127.6, 95.6, 55.6, 50.6, 28.9 ppm; HRMS-ESI calcd for $\mathrm{C}_{13} \mathrm{H}_{13} \mathrm{Cl}_{2} \mathrm{~N}_{2} \mathrm{O}_{3}[\mathrm{M}+\mathrm{H}]^{+}$ 315.0298 , found 315.0299 .

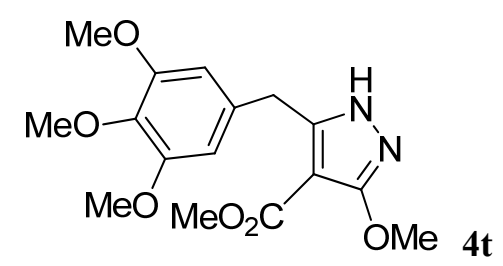

Following the general procedure, $3 \mathrm{~s}$ (108 $\mathrm{mg}, 0.2 \mathrm{mmol}$ ) was employed to afford $4 \mathbf{t}, 38 \mathrm{mg}, 57 \%$ yield, white solid, mp $193-195{ }^{\circ} \mathrm{C} ;{ }^{1} \mathrm{H}$ NMR (400 MHz, DMSO- $d_{6}$ ): $\delta 12.49$ (br s, $1 \mathrm{H}$ ), 6.62 (s, 2H), 4.07 (s, 2H), $3.81(\mathrm{~s}, 3 \mathrm{H}), 3.73$ (s, 6H), $3.71(\mathrm{~s}, 3 \mathrm{H}), 3.61$ (s, 3H) ppm; ${ }^{13} \mathrm{C}$ NMR (100 MHz, 
DMSO- $\left.d_{6}\right): \delta 163.2,162.4,152.8,147.6,136.2,133.6,105.8,94.6,59.9,55.8,55.5,50.5,31.3$ ppm; HRMS-ESI calcd for $\mathrm{C}_{16} \mathrm{H}_{21} \mathrm{~N}_{2} \mathrm{O}_{6}[\mathrm{M}+\mathrm{H}]^{+}$337.1394, found 337.1396.

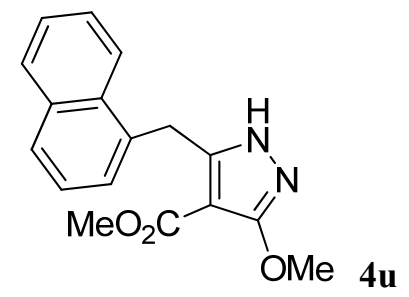

Following the general procedure, $\mathbf{3 w}$ (100 mg, $0.2 \mathrm{mmol}$ ) was employed to afford $\mathbf{4 u}, 37 \mathrm{mg}, 63 \%$ yield, white solid, mp 206-207 ${ }^{\circ} \mathrm{C} ;{ }^{1} \mathrm{H}$ NMR (400 MHz, DMSO- $d_{6}$ ): $\delta 12.42$ (br s, $1 \mathrm{H}$ ), 8.12 (d, $J$ $=7.7 \mathrm{~Hz}, 1 \mathrm{H}), 7.95(\mathrm{~d}, J=7.4 \mathrm{~Hz}, 1 \mathrm{H}), 7.82(\mathrm{~d}, J=8.1 \mathrm{~Hz}, 1 \mathrm{H}), 7.60-7.50(\mathrm{~m}, 2 \mathrm{H}), 7.43(\mathrm{t}, J=$ $7.6 \mathrm{~Hz}, 1 \mathrm{H}), 7.12(\mathrm{~d}, J=6.8 \mathrm{~Hz}, 1 \mathrm{H}), 4.65(\mathrm{~s}, 2 \mathrm{H}), 3.85(\mathrm{~s}, 3 \mathrm{H}), 3.63(\mathrm{~s}, 3 \mathrm{H}) \mathrm{ppm} ;{ }^{13} \mathrm{C}$ NMR $(100$ MHz, DMSO- $\left.d_{6}\right): \delta 163.3,162.4,147.2,133.8,133.4,131.3,128.6,127.2,126.4,125.9,125.8$, 125.7, 123.5, 95.5, 55.6, 50.6, 28.5 ppm; HRMS-ESI calcd for $\mathrm{C}_{17} \mathrm{H}_{17} \mathrm{~N}_{2} \mathrm{O}_{3}[\mathrm{M}+\mathrm{H}]^{+}$297.1234, found 297.1239 .

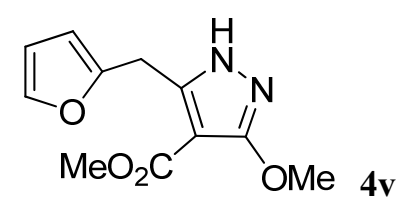

Following the general procedure, $\mathbf{3} \mathbf{x}(88 \mathrm{mg}, 0.2 \mathrm{mmol}$ ) was employed to afford $\mathbf{4 v}, 23 \mathrm{mg}, 49 \%$ yield, white solid, mp $185-186{ }^{\circ} \mathrm{C} ;{ }^{1} \mathrm{H}$ NMR (400 MHz, DMSO- $d_{6}$ ): $\delta 12.60$ (br s, $1 \mathrm{H}$ ), 7.52 (br s, 1H), 6.35 (br s, 1H), $6.08(\mathrm{~d}, J=2.6 \mathrm{~Hz}, 1 \mathrm{H}), 4.19$ (s, 2H), $3.82(\mathrm{~s}, 3 \mathrm{H}), 3.66(\mathrm{~s}, 3 \mathrm{H}) \mathrm{ppm} ;{ }^{13} \mathrm{C}$ NMR (100 MHz, DMSO- $\left.d_{6}\right): \delta 162.9,162.3,150.7,144.6,142.0,110.7,106.5,95.0,55.6,50.5$, 24.5 ppm; HRMS-ESI calcd for $\mathrm{C}_{11} \mathrm{H}_{13} \mathrm{~N}_{2} \mathrm{O}_{4}[\mathrm{M}+\mathrm{H}]^{+}$237.0870, found 237.0872.

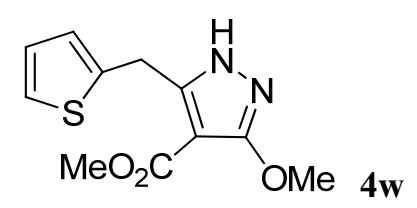

Following the general procedure, $\mathbf{3 y}(91 \mathrm{mg}, 0.2 \mathrm{mmol}$ ) was employed to afford $\mathbf{4 w}, 29 \mathrm{mg}, 58 \%$ yield, white solid, mp $134-135{ }^{\circ} \mathrm{C} ;{ }^{1} \mathrm{H}$ NMR (400 MHz, DMSO- $d_{6}$ ): $\delta 12.62$ (br s, $1 \mathrm{H}$ ), 7.32 (d, $J$ $=4.8 \mathrm{~Hz}, 1 \mathrm{H}), 6.94-6.90(\mathrm{~m}, 2 \mathrm{H}), 4.33(\mathrm{~s}, 2 \mathrm{H}), 3.82(\mathrm{~s}, 3 \mathrm{H}), 3.69(\mathrm{~s}, 3 \mathrm{H}) \mathrm{ppm} ;{ }^{13} \mathrm{C}$ NMR $(100$ MHz, DMSO- $\left.d_{6}\right): \delta 162.9,162.3,147.0,140.0,126.9,125.7,124.8,94.6,55.6,50.5,25.6$ ppm; HRMS-ESI calcd for $\mathrm{C}_{11} \mathrm{H}_{13} \mathrm{~N}_{2} \mathrm{O}_{3} \mathrm{~S}[\mathrm{M}+\mathrm{H}]^{+}$253.0641, found 253.0639. 


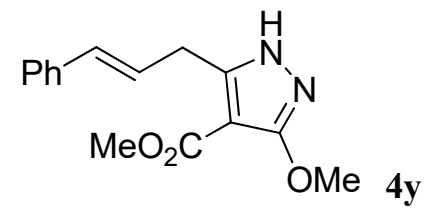

Following the general procedure, $\mathbf{3 A}$ (95 mg, $0.2 \mathrm{mmol}$ ) was employed to afford $\mathbf{4 y}, 28 \mathrm{mg}, 51 \%$ yield, white solid, mp $112-113{ }^{\circ} \mathrm{C} ;{ }^{1} \mathrm{H}$ NMR (400 MHz, DMSO- $d_{6}$ ): $\delta 12.48$ (br s, $1 \mathrm{H}$ ), 7.39 (d, $J$ $=7.4 \mathrm{~Hz}, 2 \mathrm{H}), 7.31(\mathrm{t}, J=7.5 \mathrm{~Hz}, 2 \mathrm{H}), 7.22(\mathrm{t}, J=7.2 \mathrm{~Hz}, 1 \mathrm{H}), 6.47$ (d, $J=15.9 \mathrm{~Hz}, 1 \mathrm{H}), 6.41-$ $6.32(\mathrm{~m}, 1 \mathrm{H}), 3.83(\mathrm{~s}, 3 \mathrm{H}), 3.73(\mathrm{~d}, J=6.3 \mathrm{~Hz}, 2 \mathrm{H}), 3.69$ (s, 3H) ppm; ${ }^{13} \mathrm{C}$ NMR (100 MHz, DMSO- $d_{6}$ ): $163.0,162.4,147.0,136.7,131.7,128.6,127.4,126.1,125.3,94.7,55.5,50.5,29.2$ ppm; HRMS-ESI calcd for $\mathrm{C}_{15} \mathrm{H}_{17} \mathrm{~N}_{2} \mathrm{O}_{3}[\mathrm{M}+\mathrm{H}]^{+}$273.1234, found 273.1239.

\section{Reference}

6. Guillou, S.; Janin, Y. L. Chem. Eur. J. 2010, 16, 4669-4677. 


\section{ORTEP Drawings for Compounds 3a and $4 \mathrm{~h}$}

Figure S1. ORTEP Drawing for 3a with 50\% ellipsoid probability

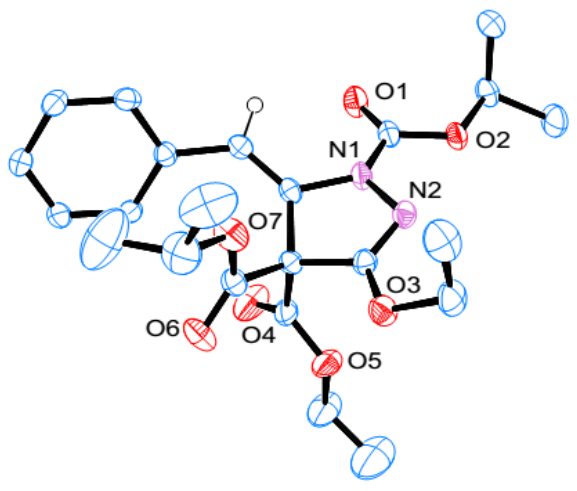

Table S1. Crystal data and structure refinement for $\mathbf{3 a}$

\begin{tabular}{|c|c|}
\hline Identification code & $3 \mathbf{a}$ \\
\hline Empirical formula & $\mathrm{C}_{23} \mathrm{H}_{30} \mathrm{~N}_{2} \mathrm{O}_{7}$ \\
\hline Formula weight & 446.49 \\
\hline Temperature & $113(2) \mathrm{K}$ \\
\hline Wavelength & $0.71073 \AA$ \\
\hline Crystal system, Space group & Monoclinic, $\mathrm{P} 2(1) / \mathrm{n}$ \\
\hline & $\mathrm{a}=9.0774(18) \AA, \alpha=90^{\circ}$ \\
\hline Unit cell dimensions & $\mathrm{b}=15.150(3) \AA, \beta=93.62(3)^{\circ}$ \\
\hline & $\mathrm{c}=16.882(3) \AA, \gamma=90^{\circ}$ \\
\hline Volume,Z & $2317.0(8) \AA^{3}$ \\
\hline $\mathrm{Z}$, Calculated density & $4,1.280 \mathrm{Mg} / \mathrm{m}^{3}$ \\
\hline Absorption coefficient & $0.095 \mathrm{~mm}^{-1}$ \\
\hline $\mathrm{F}(000)$ & 952 \\
\hline Crystal size & $0.20 \times 0.18 \times 0.12 \mathrm{~mm}^{3}$ \\
\hline$\theta$ range for data collection & 1.81 to $25.01^{\circ}$ \\
\hline Limiting indices & $-10<=\mathrm{h}<=10,-18<=\mathrm{k}<=17,-20<=\mathrm{l}<=19$ \\
\hline Reflections collected / unique & $15767 / 4065[\mathrm{R}(\mathrm{int})=0.0555]$ \\
\hline Completeness to $\theta=27.88^{\circ}$ & $99.8 \%$ \\
\hline Absorption correction & Semi-empirical from equivalents \\
\hline Max. and min. transmission & 0.9887 and 0.9813 \\
\hline Refinement method & Full-matrix least-squares on $\mathrm{F}^{2}$ \\
\hline Data / restraints / parameters & $4065 / 0 / 296$ \\
\hline Goodness-of-fit on $\mathrm{F}^{2}$ & 1.040 \\
\hline Final $R$ indices $[I>2 \sigma(I)]$ & $\mathrm{R}_{1}=0.0661, \mathrm{wR}_{2}=0.1930$ \\
\hline $\mathrm{R}$ indices (all data) & $\mathrm{R}_{1}=0.0824, \mathrm{wR}_{2}=0.2099$ \\
\hline Extinction coefficient & $0.039(5)$ \\
\hline Largest diff. peak and hole & 0.222 and -0.252 e. $\AA^{-3}$ \\
\hline
\end{tabular}


Figure S2. ORTEP Drawing for $\mathbf{4 h}$ with 50\% ellipsoid probability

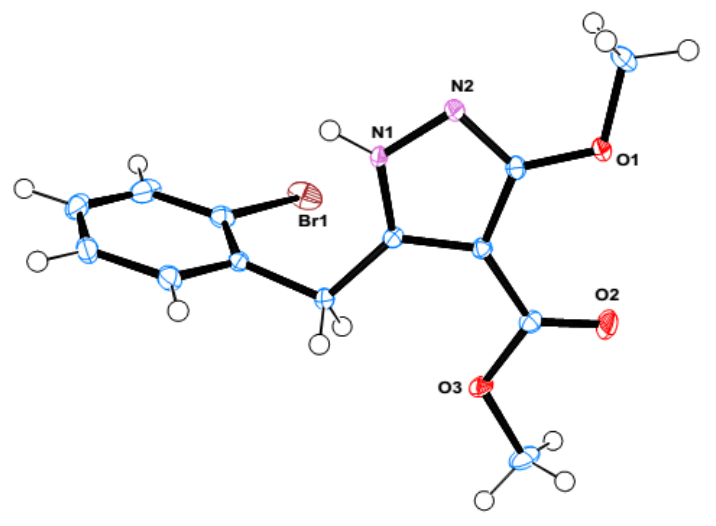

Table S2. Crystal data and structure refinement for $\mathbf{4 h}$

\section{Identification code \\ Empirical formula \\ Formula weight \\ Temperature \\ Wavelength}

Crystal system, Space group

Unit cell dimensions

Volume,Z

$Z$, Calculated density

Absorption coefficient

$\mathrm{F}(000)$

Crystal size

$\theta$ range for data collection

Limiting indices

Reflections collected / unique

Completeness to $\theta=27.88^{\circ}$

Absorption correction

Max. and min. transmission

Refinement method

Data / restraints / parameters

Goodness-of-fit on $\mathrm{F}^{2}$

Final $R$ indices $[\mathrm{I}>2 \sigma(\mathrm{I})]$

$\mathrm{R}$ indices (all data)

Largest diff. peak and hole
$4 h$

$$
\mathrm{C}_{13} \mathrm{H}_{13} \mathrm{BrN}_{2} \mathrm{O}_{3}
$$

325.16

113(2) K

$0.71073 \AA$

Triclinic, $\mathrm{P}-1$

$$
\begin{aligned}
& \mathrm{a}=8.296(3) \AA, \alpha=100.061(2)^{\circ} \\
& \mathrm{b}=8.793(3) \AA, \beta=106.015(4)^{\circ} \\
& \mathrm{c}=10.130(3) \AA, \gamma=108.472(2)^{\circ}
\end{aligned}
$$$$
\text { 645.2(3) } \AA^{3}
$$

$2,1.674 \mathrm{Mg} / \mathrm{m}^{3}$

$3.191 \mathrm{~mm}^{-1}$

328

$0.20 \times 0.18 \times 0.12 \mathrm{~mm}^{3}$

3.52 to $27.54 \mathrm{deg}$.

$$
\begin{gathered}
-10<=\mathrm{h}<=10,-11<=\mathrm{k}<=10,-13<=\mathrm{l}<=13 \\
7002 / 2920[\mathrm{R}(\text { int })=0.0223] \\
98.3 \%
\end{gathered}
$$

Semi-empirical from equivalents

0.7007 and 0.5678

Full-matrix least-squares on $\mathrm{F}^{2}$

$2920 / 0 / 178$

$$
1.078
$$

$\mathrm{R}_{1}=0.0227, \mathrm{wR}_{2}=0.0611$

$\mathrm{R}_{1}=0.0245, \mathrm{wR}_{2}=0.0614$

0.676 and -0.679 e. $\AA^{-3}$ 
IX. NMR Spectra for New Compounds
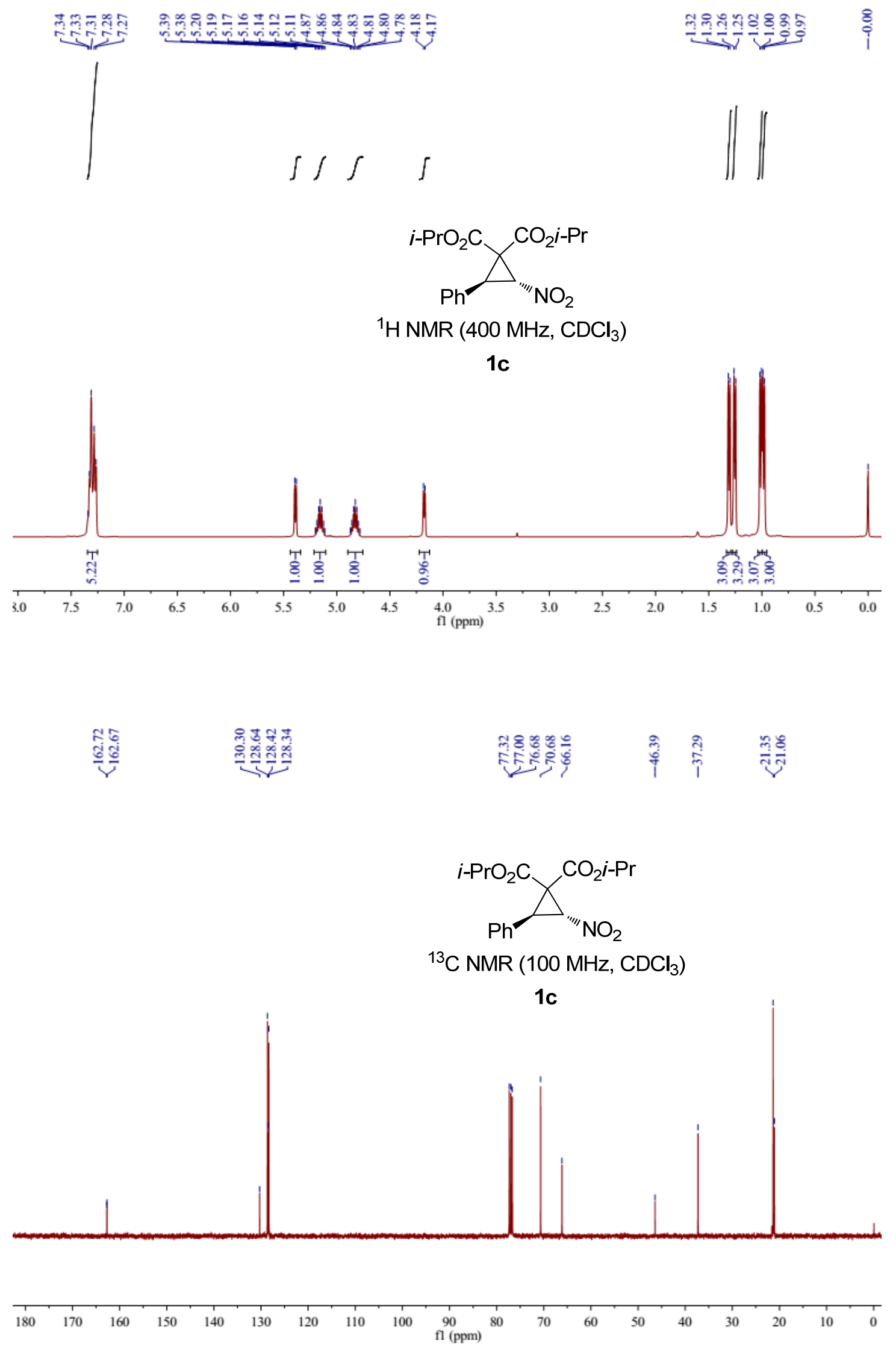


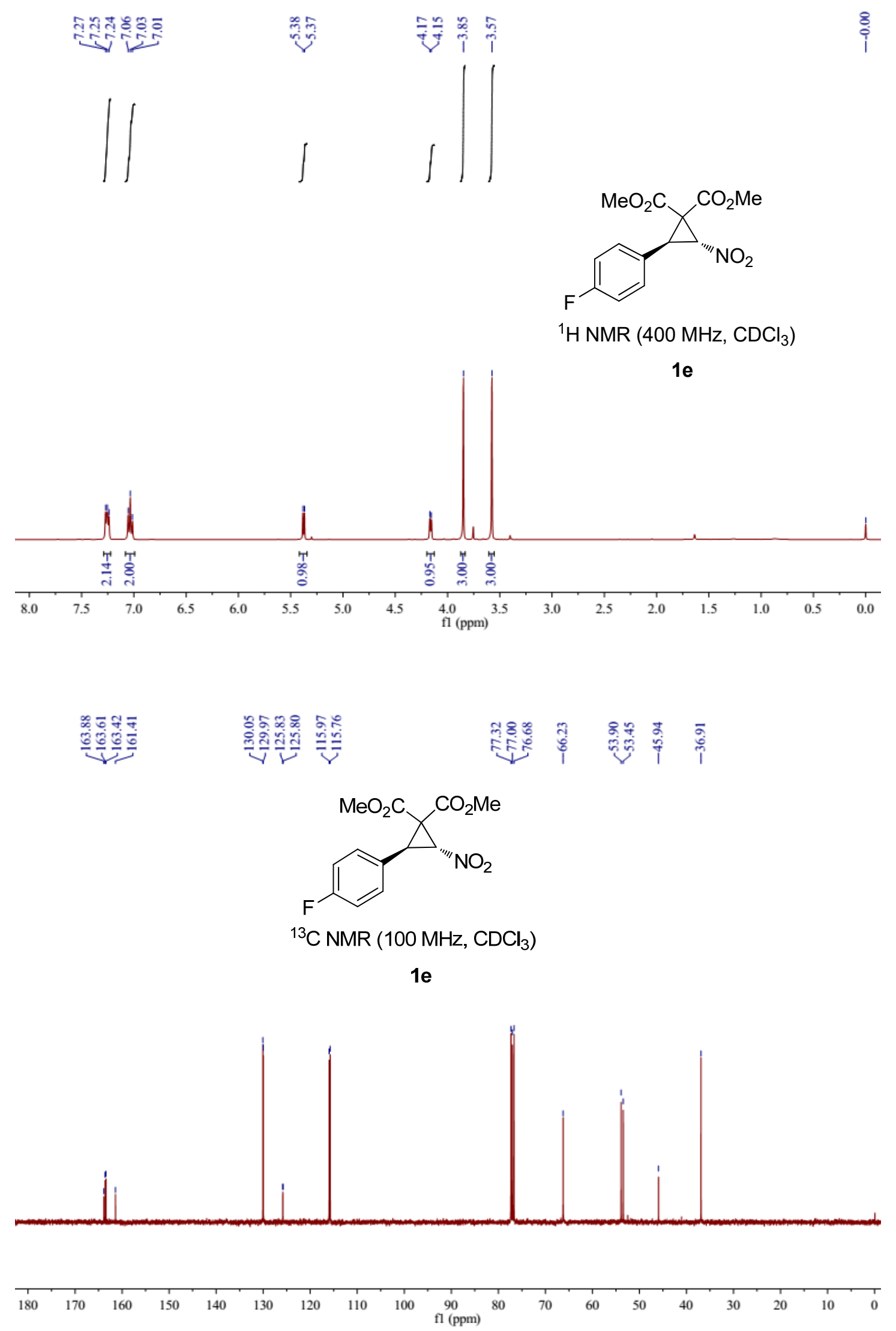




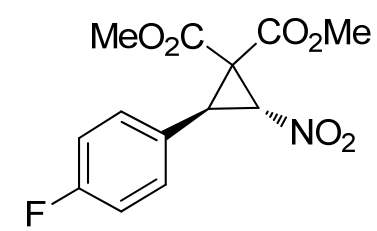

${ }^{19} \mathrm{~F} \mathrm{NMR}\left(376 \mathrm{MHz}, \mathrm{CDCl}_{3}\right.$ )

$1 e$
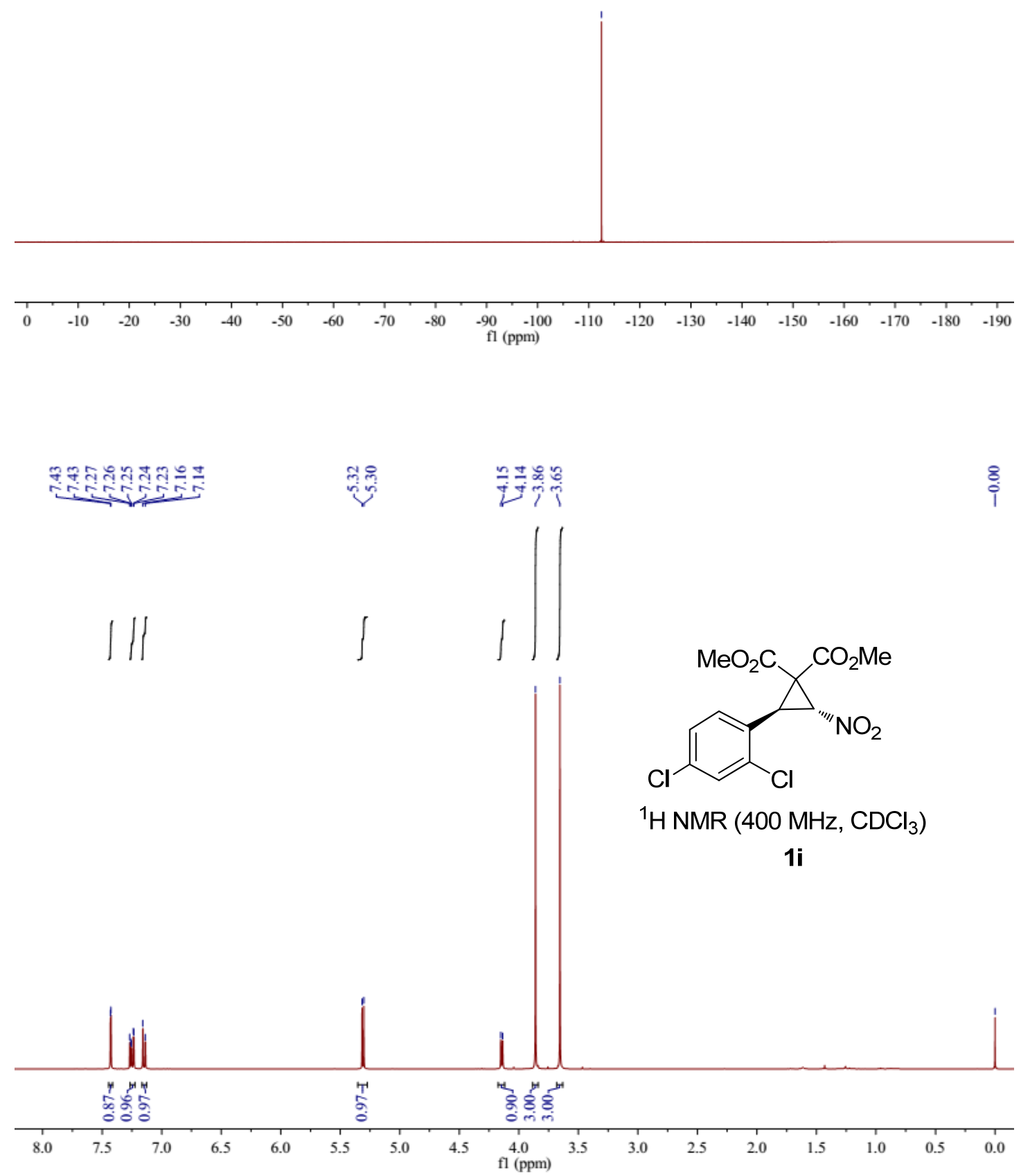


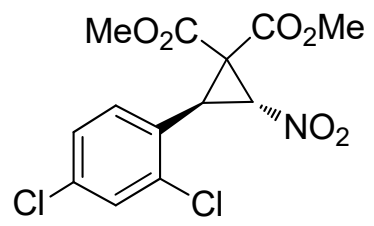

${ }^{13} \mathrm{C}$ NMR $\left(100 \mathrm{MHz}, \mathrm{CDCl}_{3}\right)$

$1 \mathbf{i}$

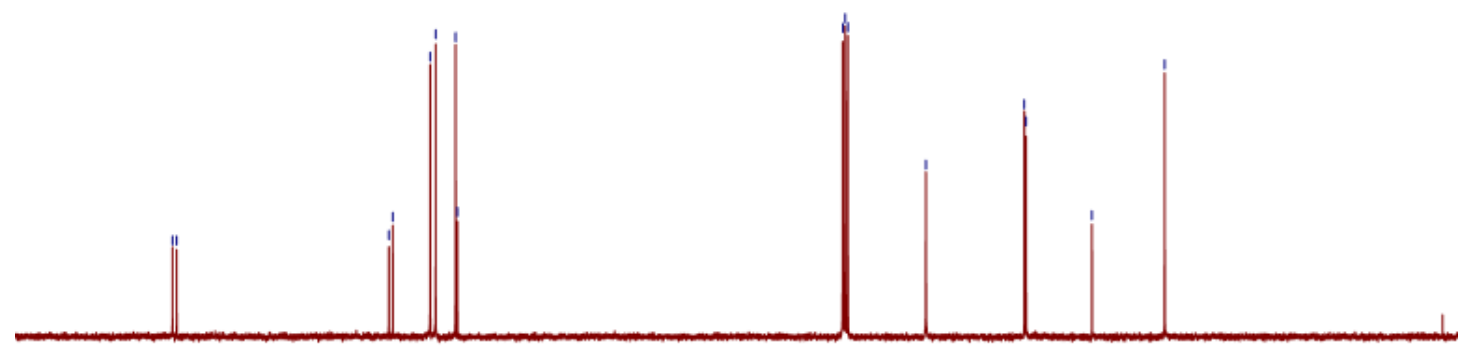

$\begin{array}{rllllllllllllllllll}180 & 170 & 160 & 150 & 140 & 130 & 120 & 110 & 100 & \begin{array}{c}90 \\ \mathrm{fl}(\mathrm{ppm})\end{array} & 80 & 70 & 60 & 50 & 40 & 30 & 20 & 10 & 0\end{array}$

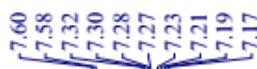

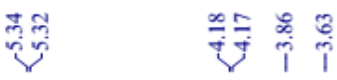

$\int \mid \|$

$\iint$

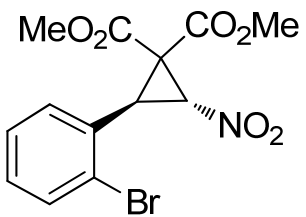

${ }^{1} \mathrm{H}$ NMR (400 MHz, $\mathrm{CDCl}_{3}$ )

1j

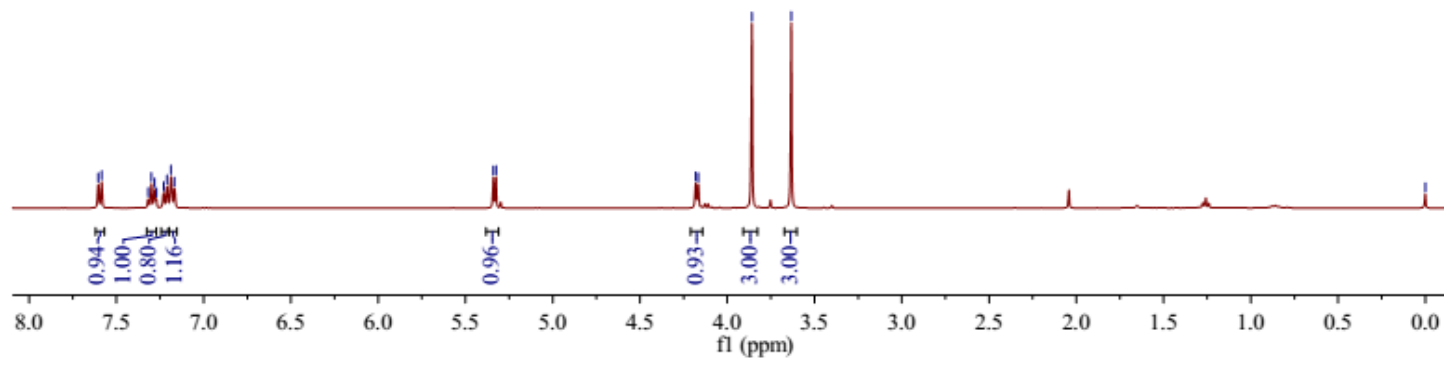



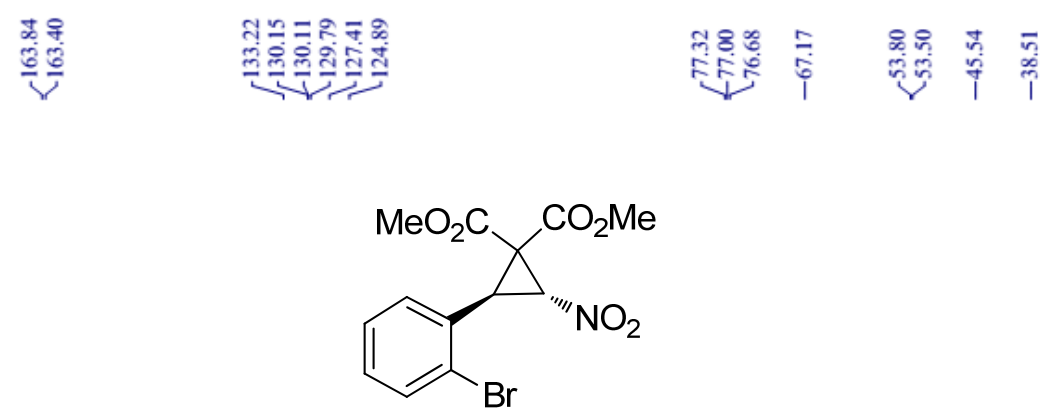

${ }^{13} \mathrm{C}$ NMR (100 MHz, $\mathrm{CDCl}_{3}$ )

1j
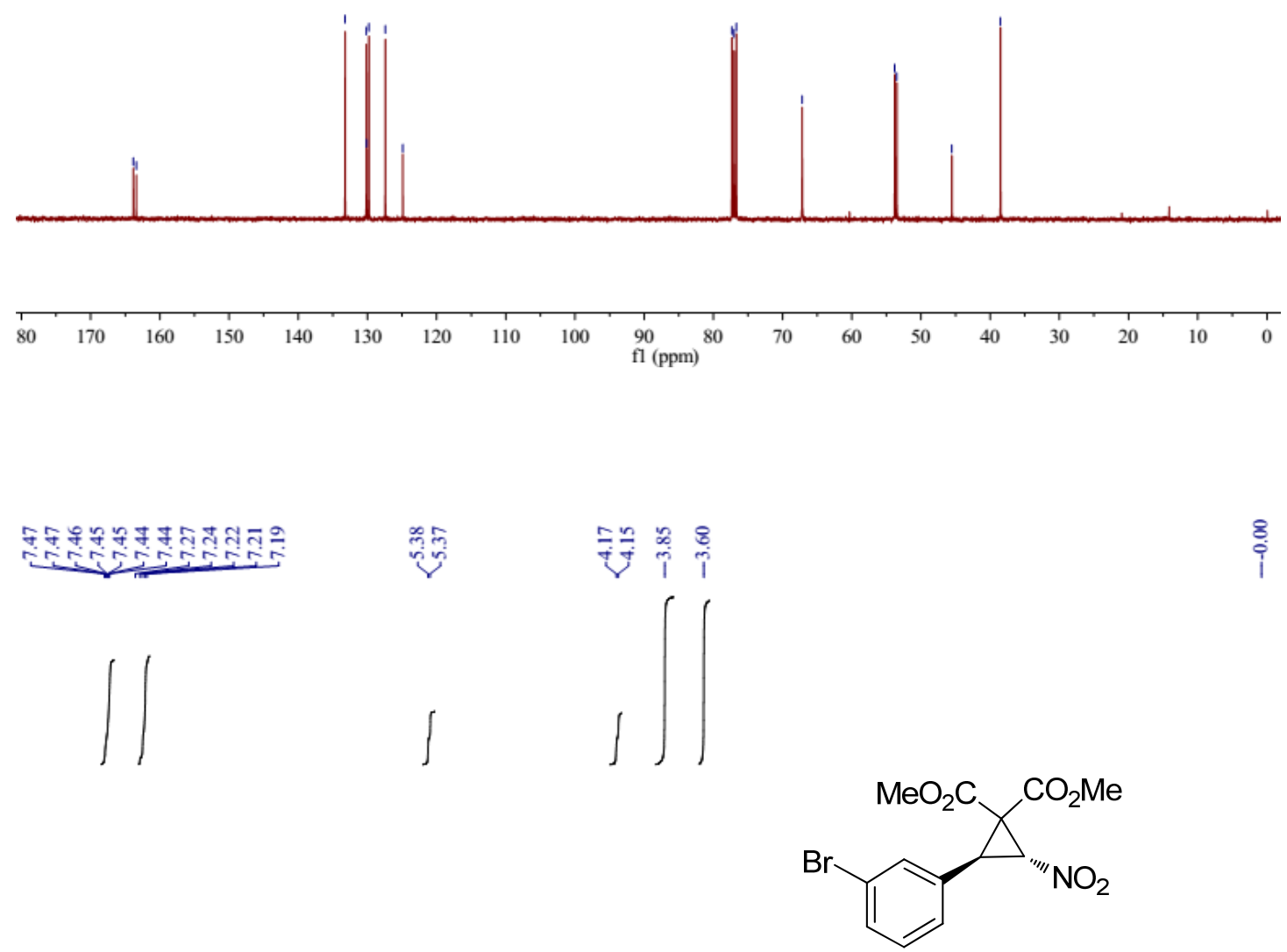

${ }^{1} \mathrm{H} \mathrm{NMR}\left(400 \mathrm{MHz}, \mathrm{CDCl}_{3}\right)$

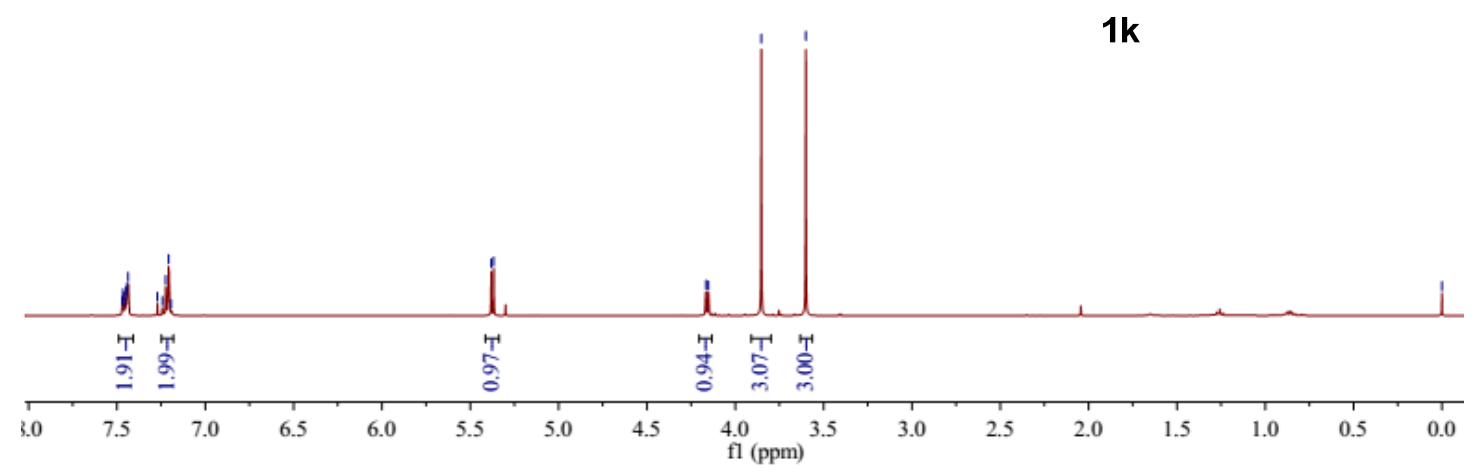




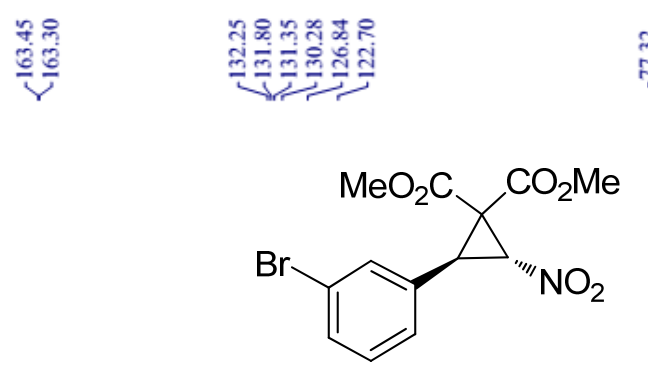

${ }^{13} \mathrm{C}$ NMR $\left(100 \mathrm{MHz}, \mathrm{CDCl}_{3}\right)$

$1 \mathrm{k}$

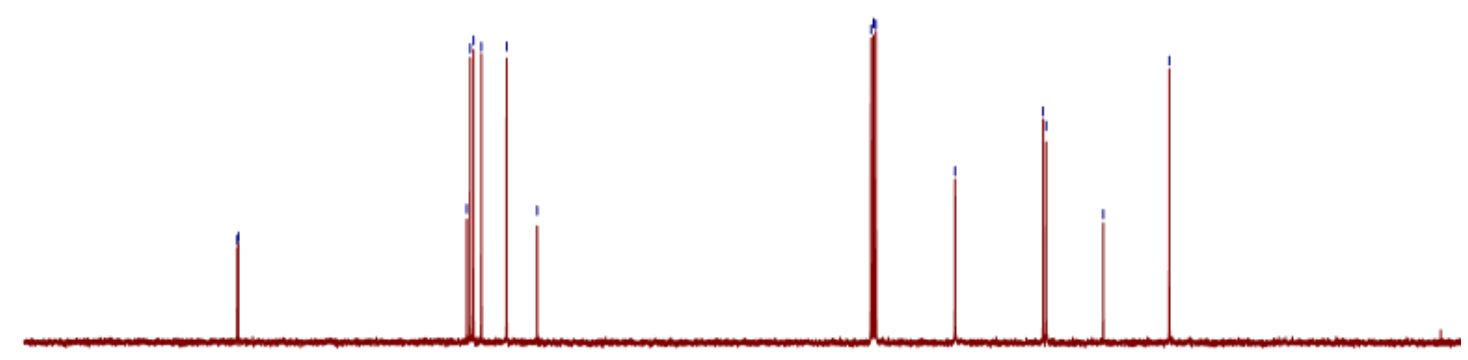

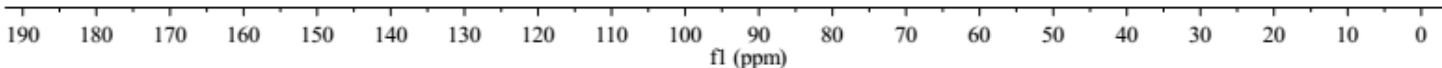

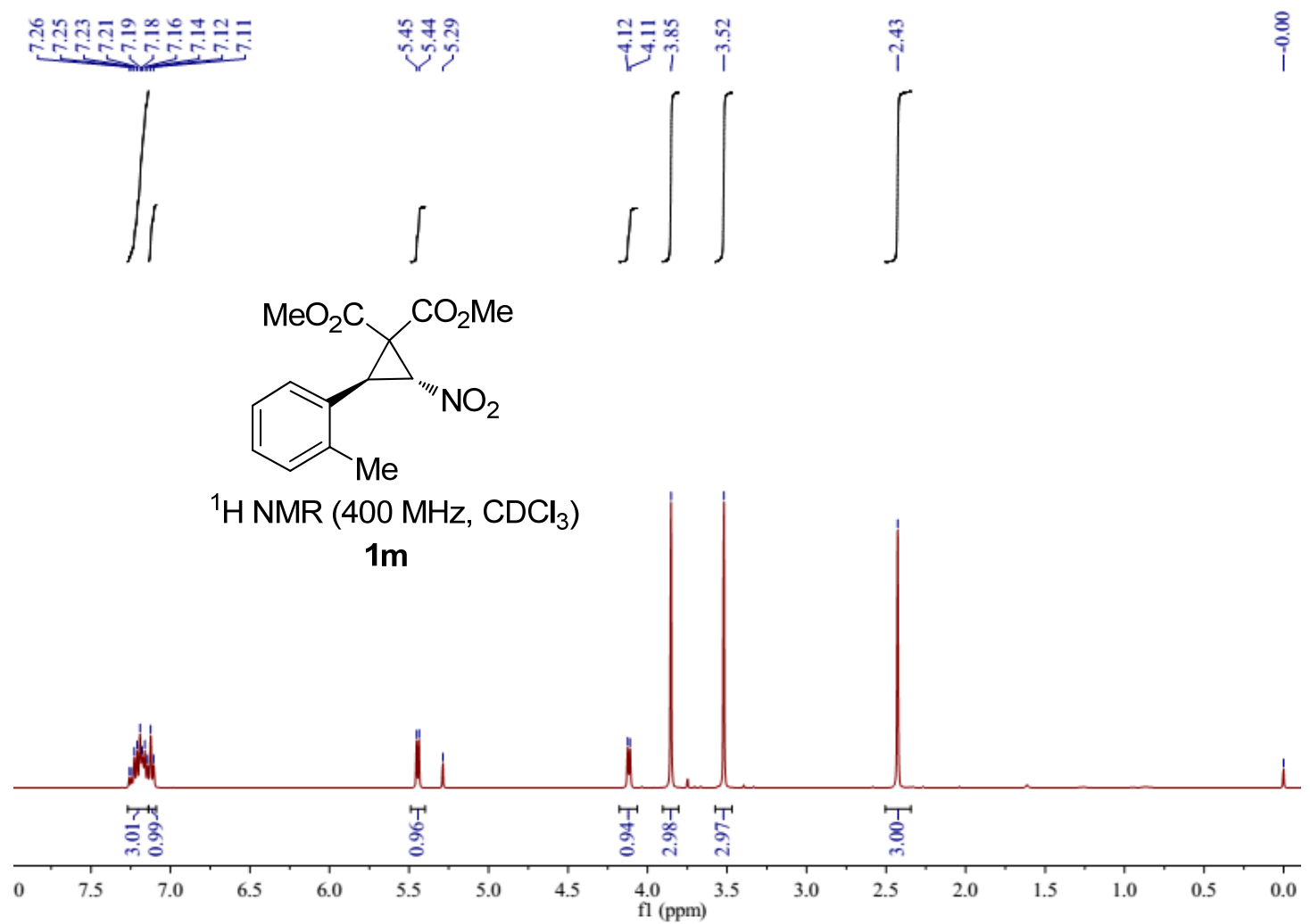




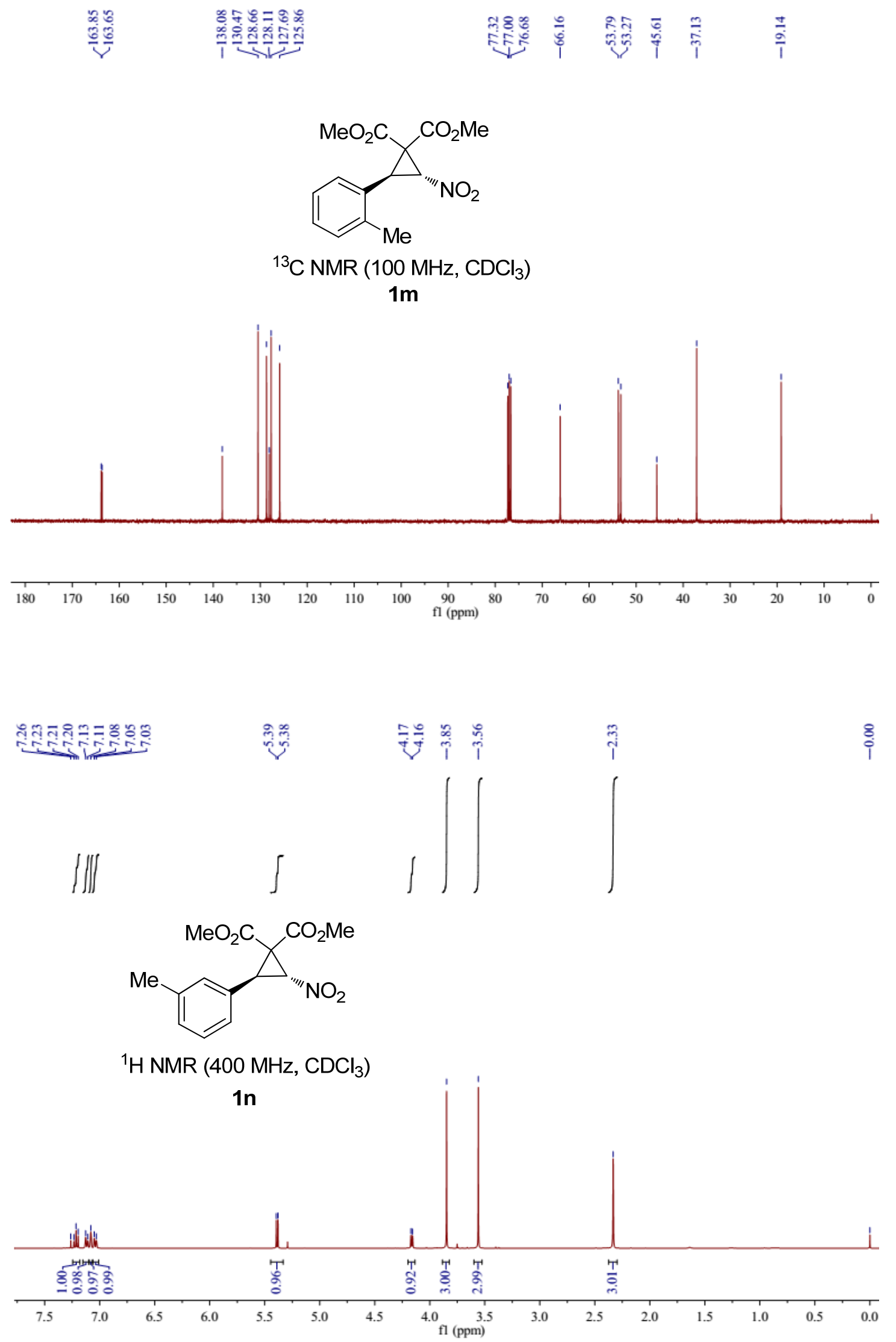



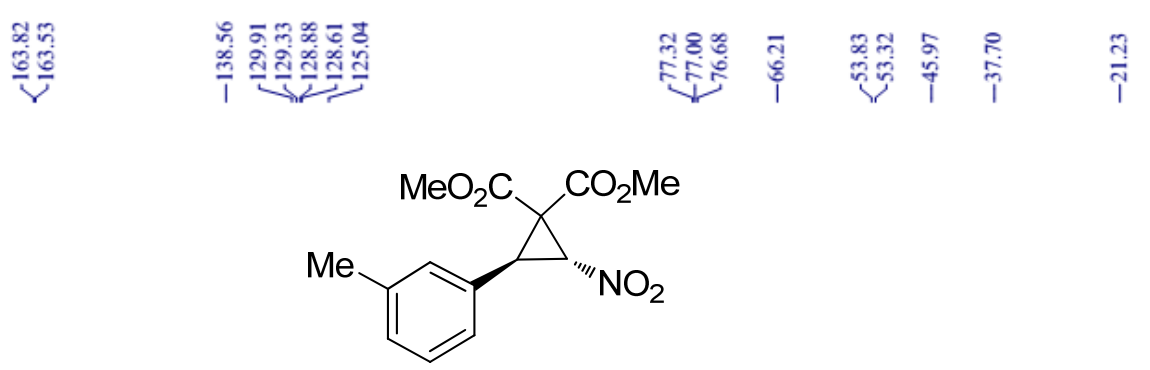

${ }^{13} \mathrm{C}$ NMR $\left(100 \mathrm{MHz}, \mathrm{CDCl}_{3}\right)$

1n
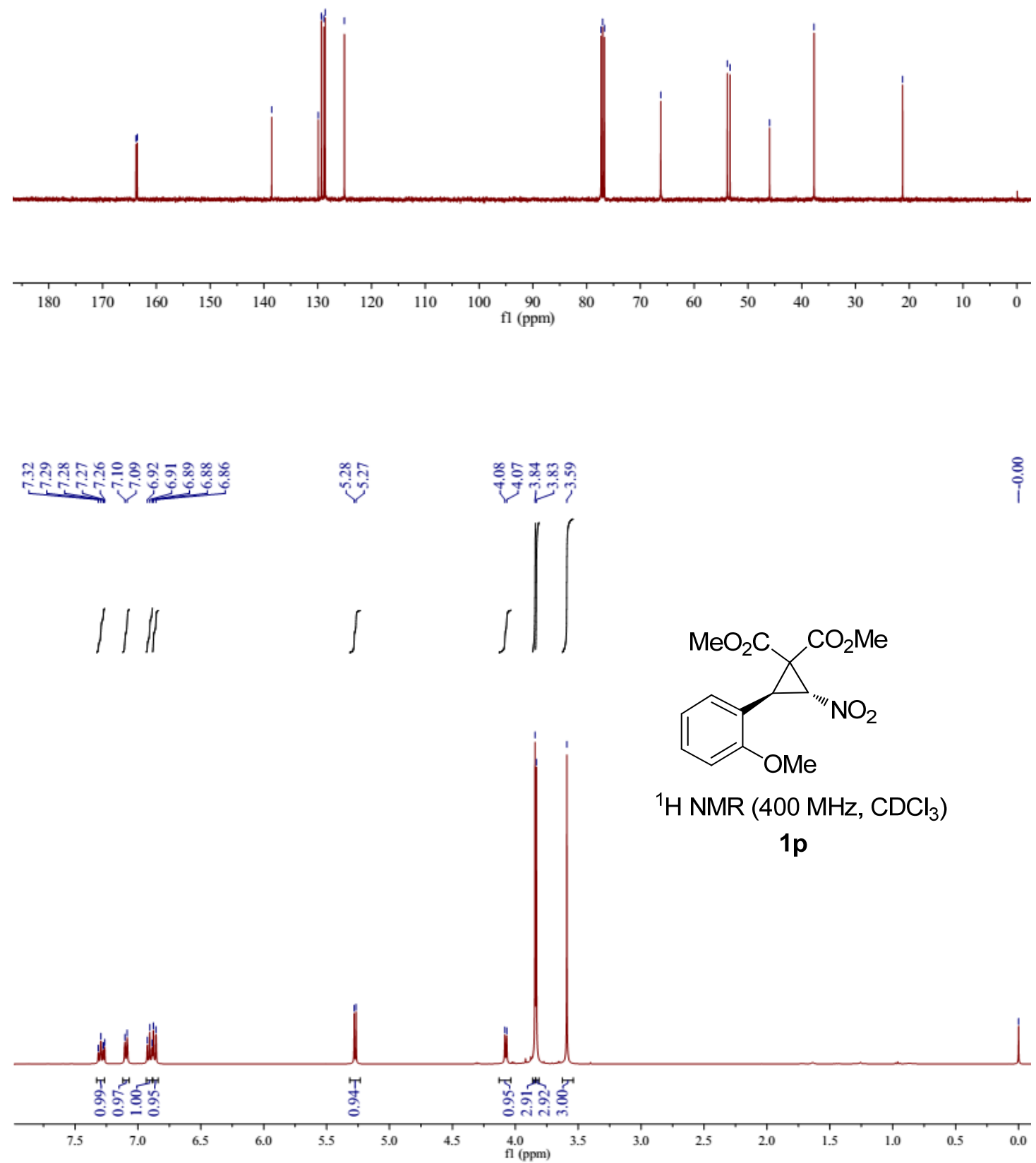


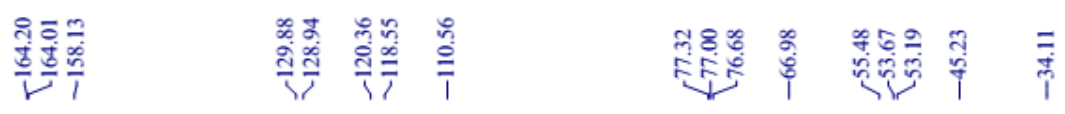

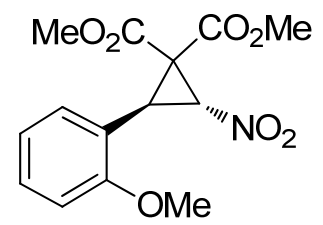

${ }^{13} \mathrm{C}$ NMR (100 MHz, $\left.\mathrm{CDCl}_{3}\right)$

$1 p$
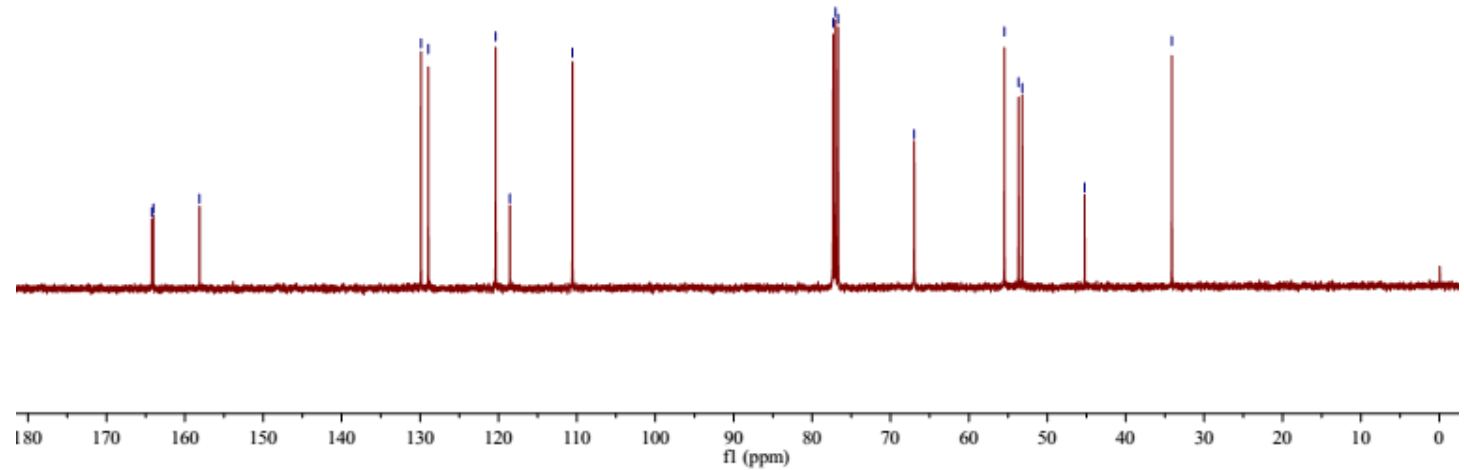

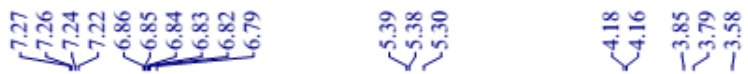

$1 \int$

\|\|

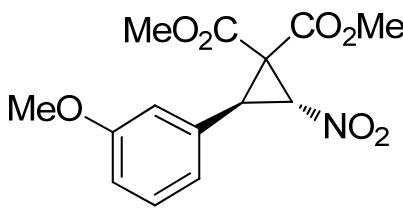

${ }^{1} \mathrm{H}$ NMR (400 MHz, $\left.\mathrm{CDCl}_{3}\right)$

$1 q$

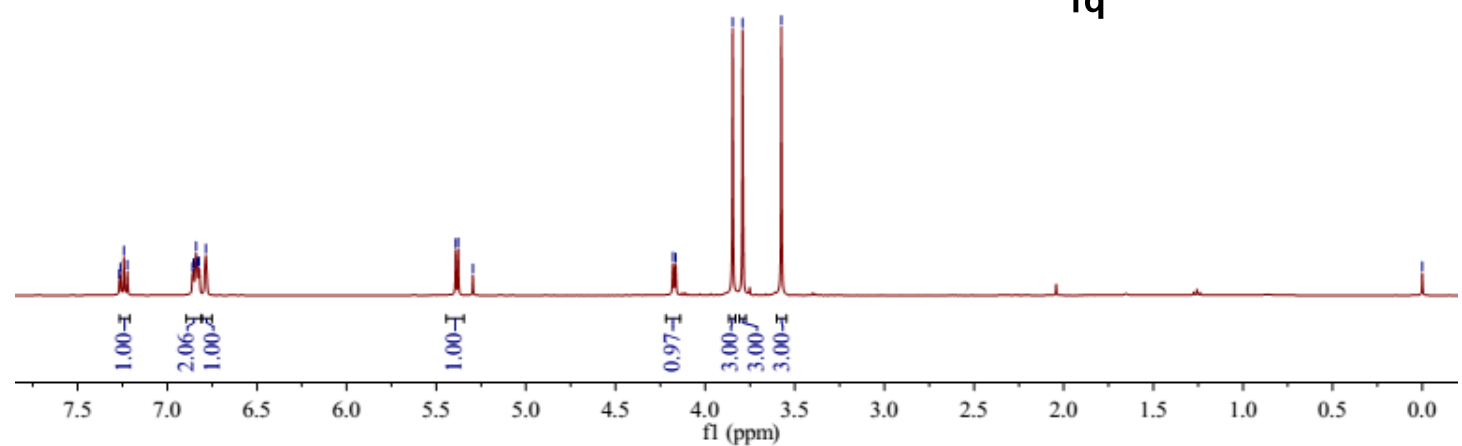




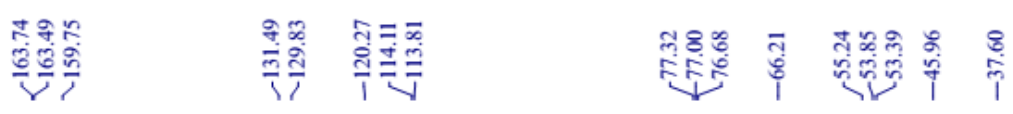

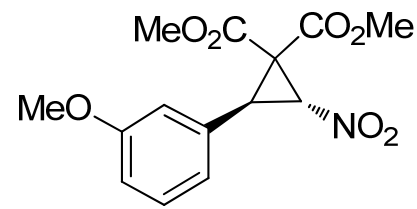

${ }^{13} \mathrm{C}$ NMR (100 MHz, $\left.\mathrm{CDCl}_{3}\right)$

$1 q$
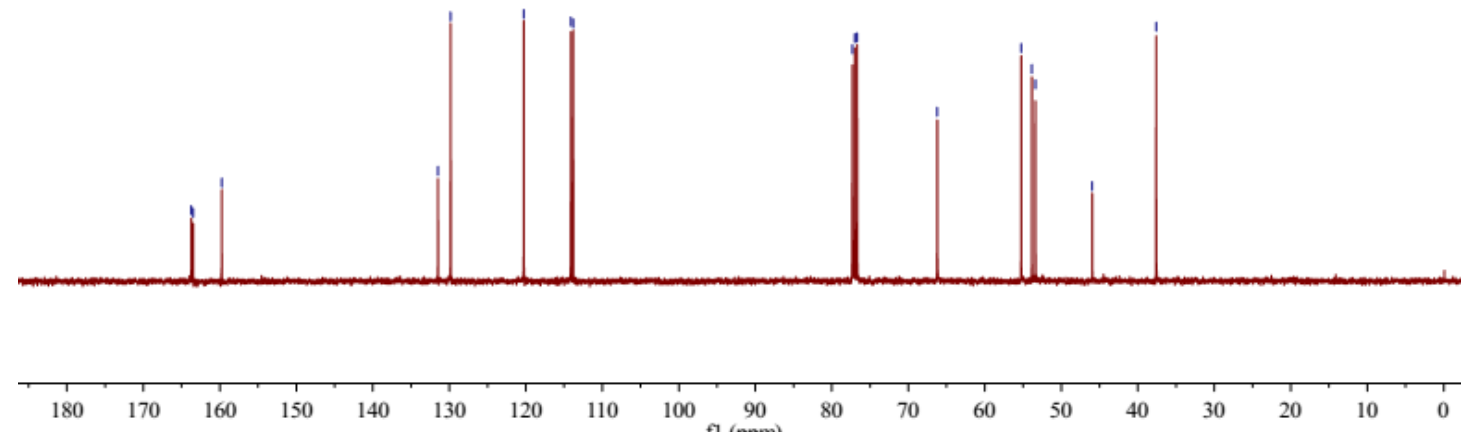

तै ।

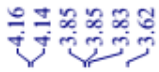

i

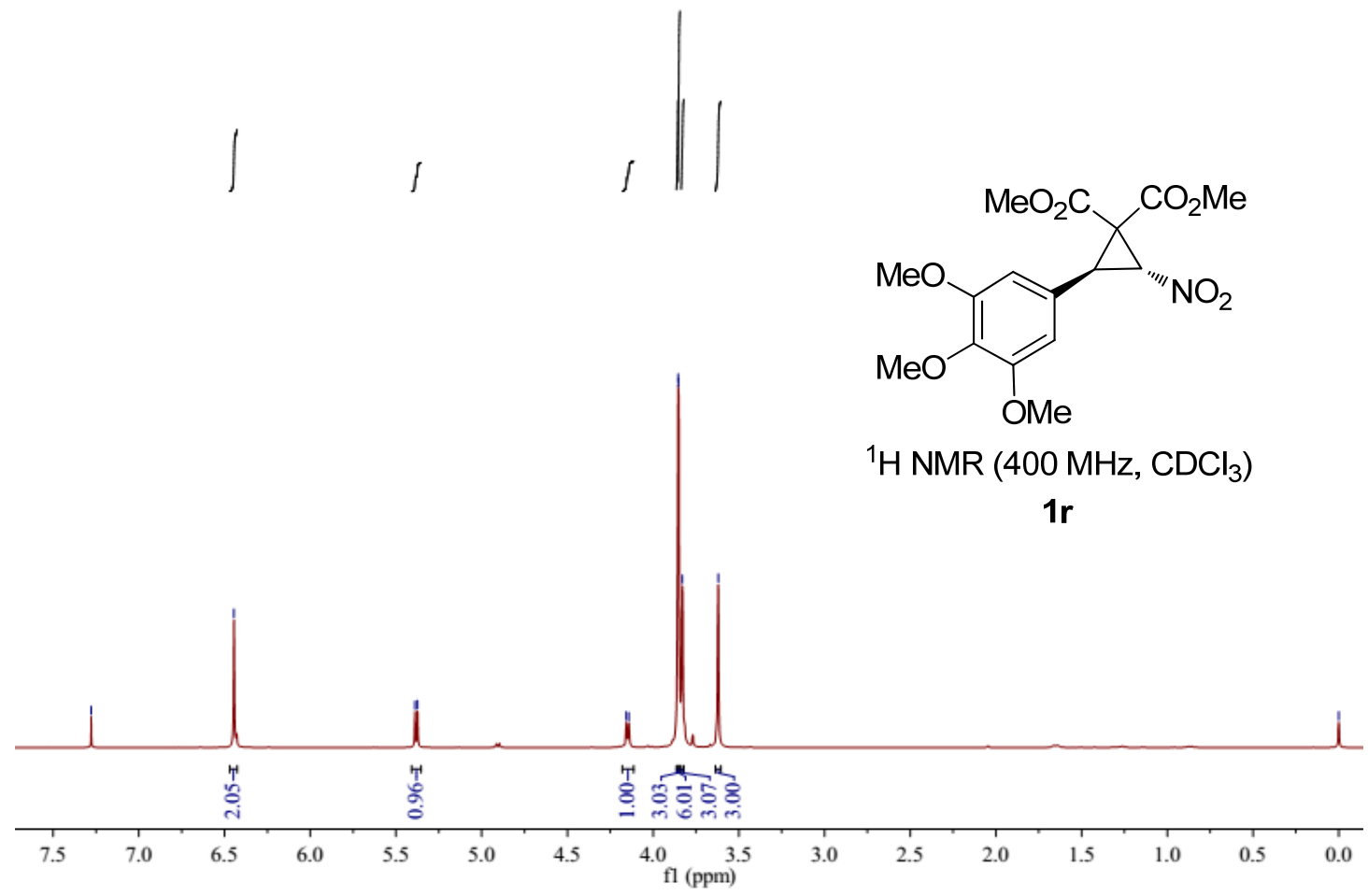




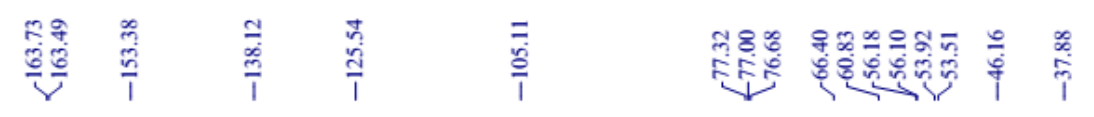

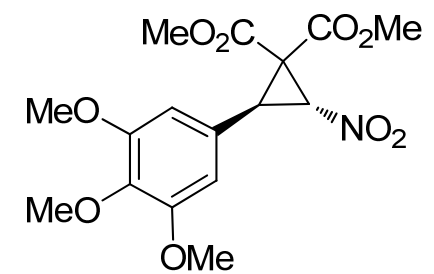

${ }^{13} \mathrm{C}$ NMR $\left(100 \mathrm{MHz}, \mathrm{CDCl}_{3}\right)$

$1 \mathbf{r}$
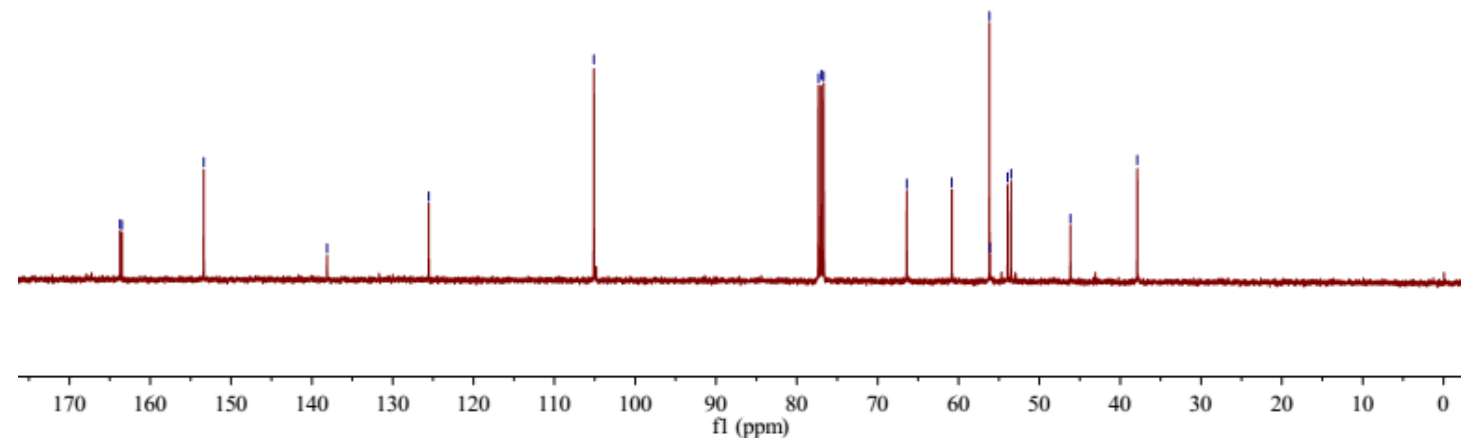

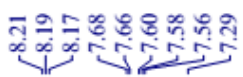

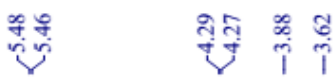

$\stackrel{8}{\circ}$

$\| \iint$
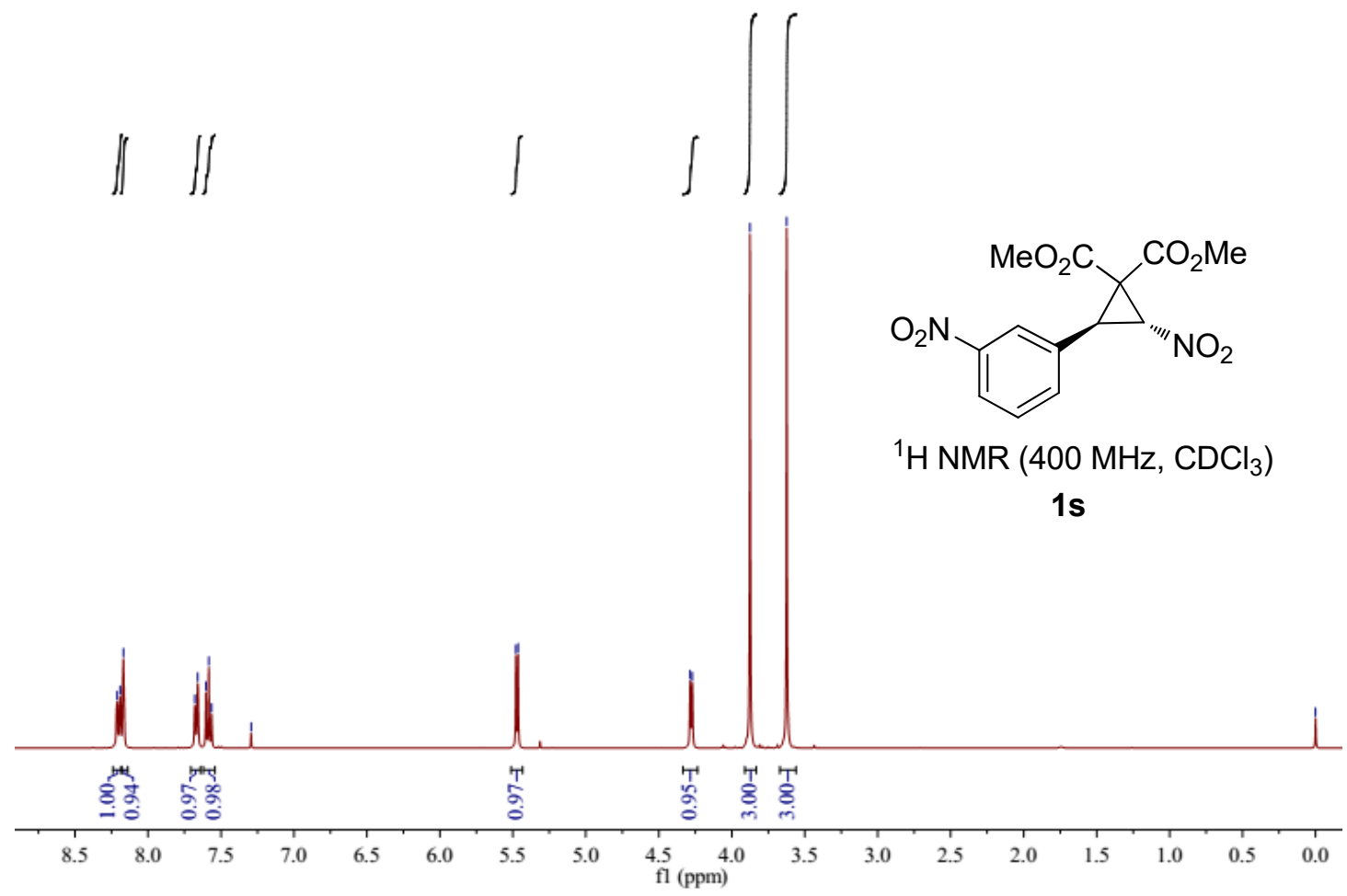


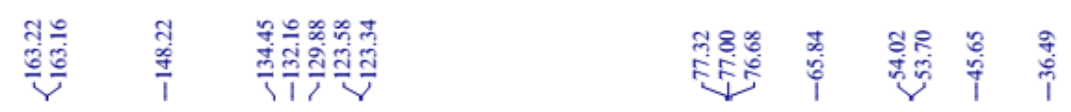

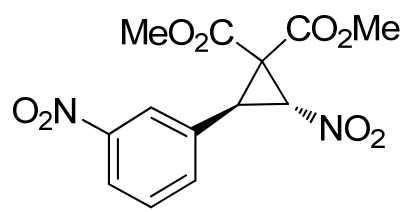

${ }^{13} \mathrm{C}$ NMR (100 MHz, $\mathrm{CDCl}_{3}$ )

$1 \mathrm{~s}$
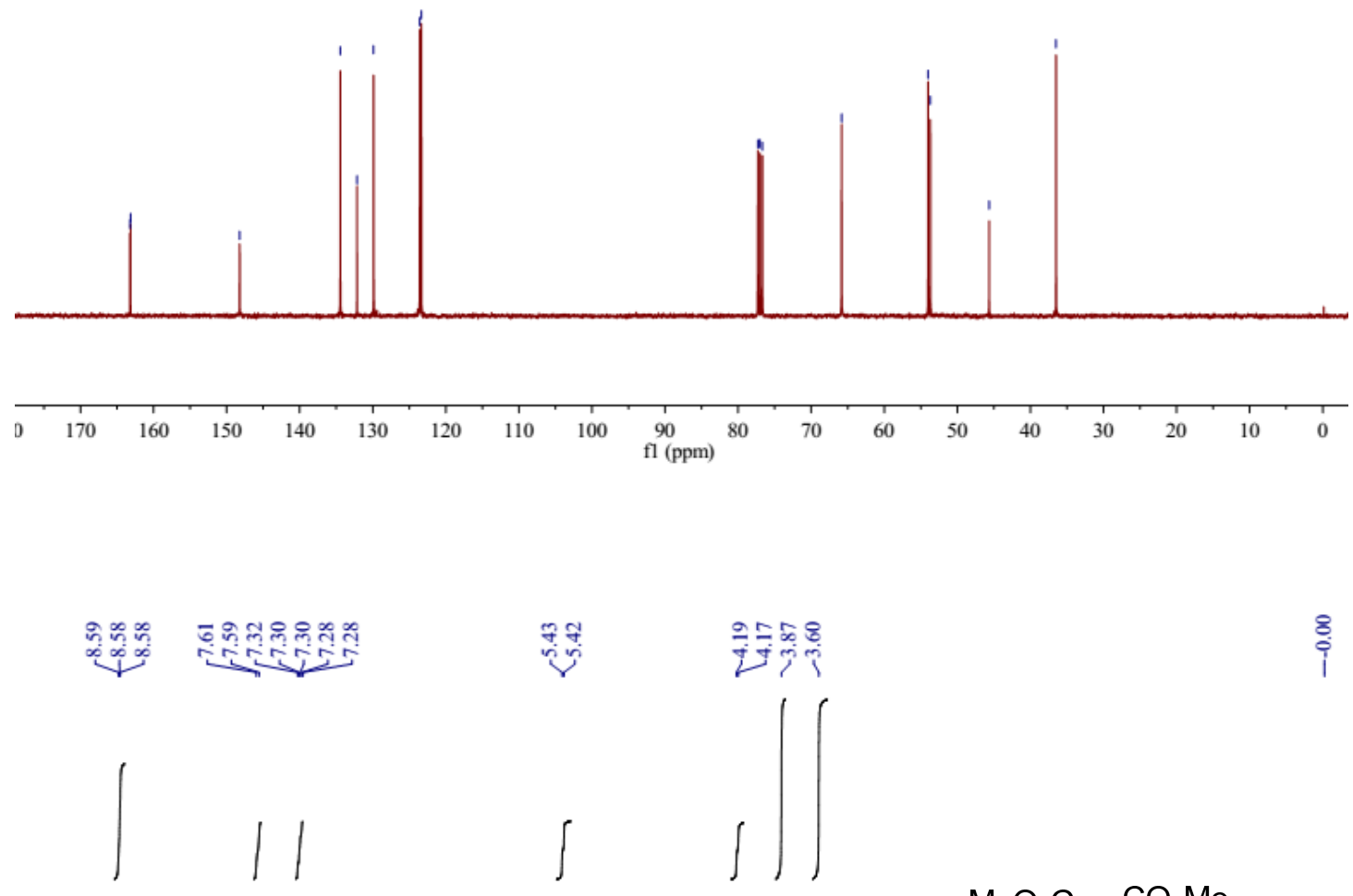

$\stackrel{8}{9}$

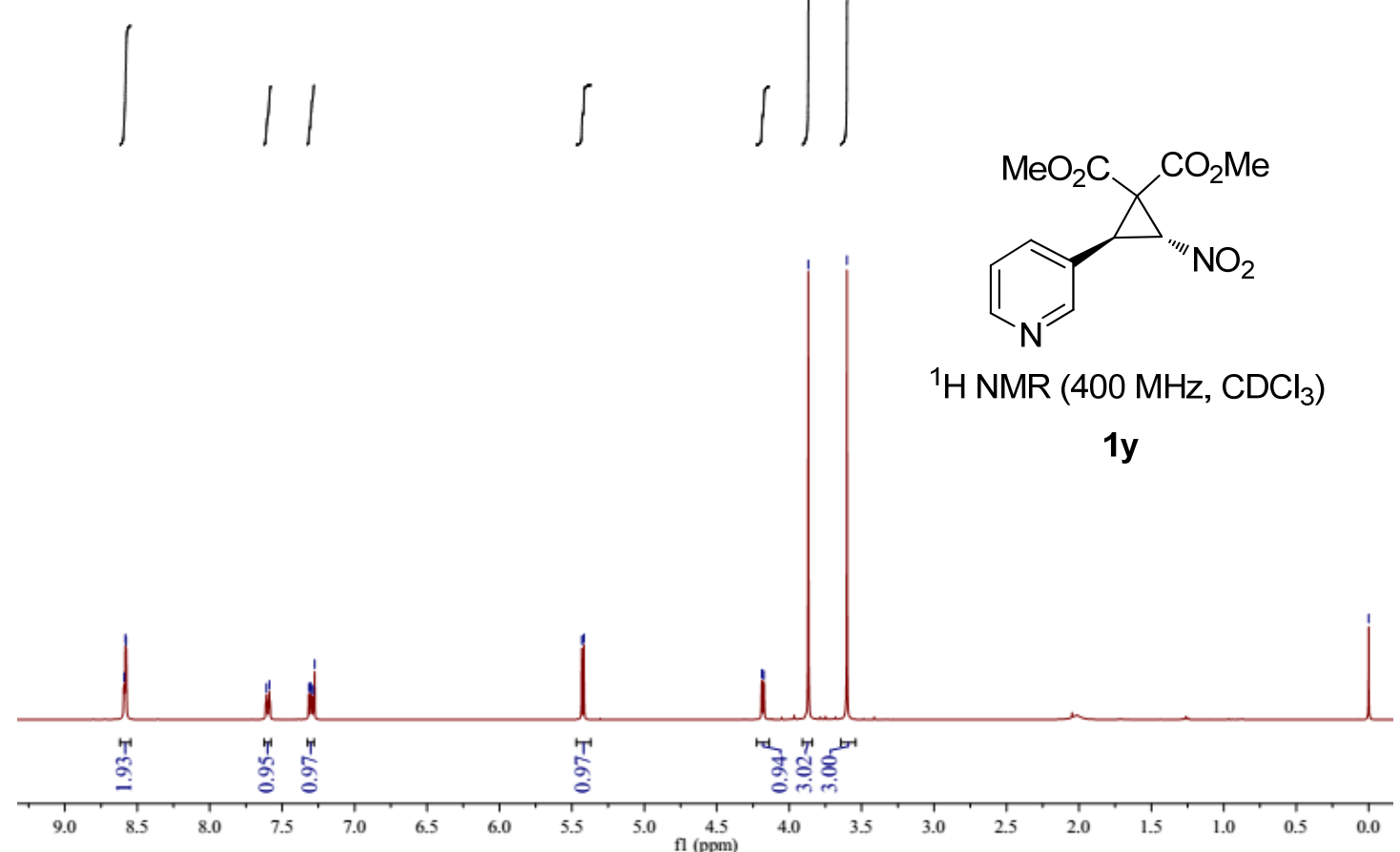




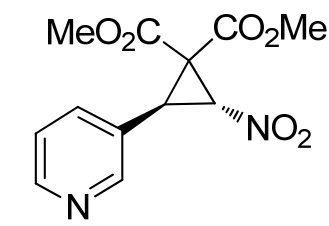

${ }^{13} \mathrm{C}$ NMR (100 MHz, $\left.\mathrm{CDCl}_{3}\right)$

$1 y$
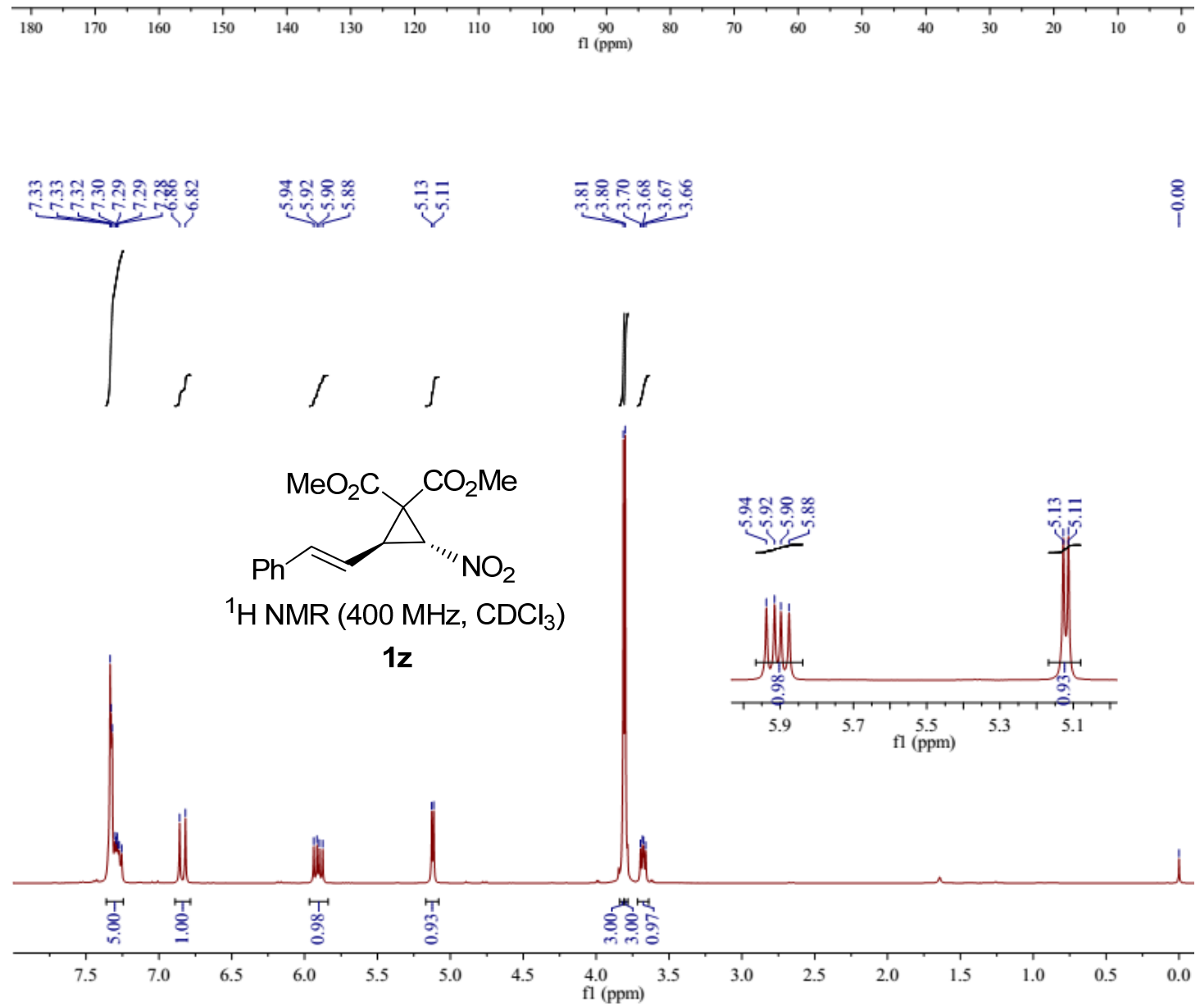

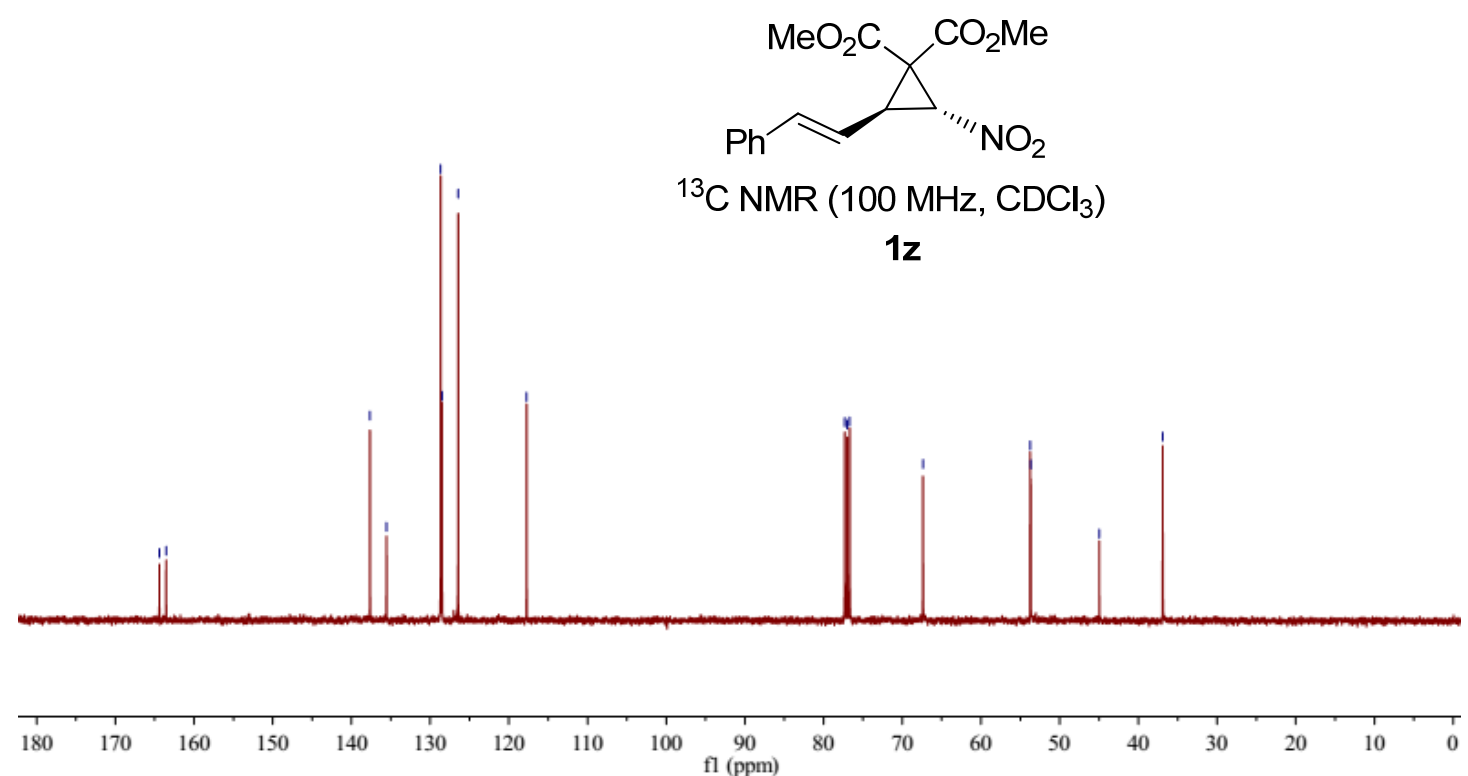

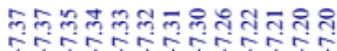

| 1

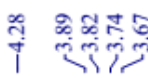

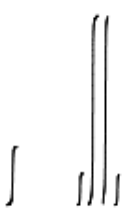

ชุ.

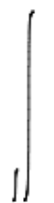

$\stackrel{8}{i}$<smiles>COC(=O)C1(C(C)=O)C(c2ccccc2)C1[N+](=O)[O-]</smiles>

${ }^{1} \mathrm{H}$ NMR $\left(400 \mathrm{MHz}, \mathrm{CDCl}_{3}\right)$

$1 \mathrm{C}$

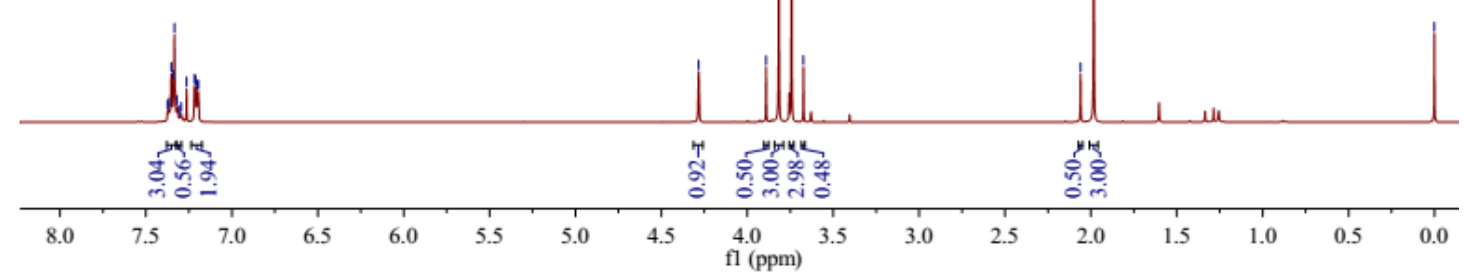




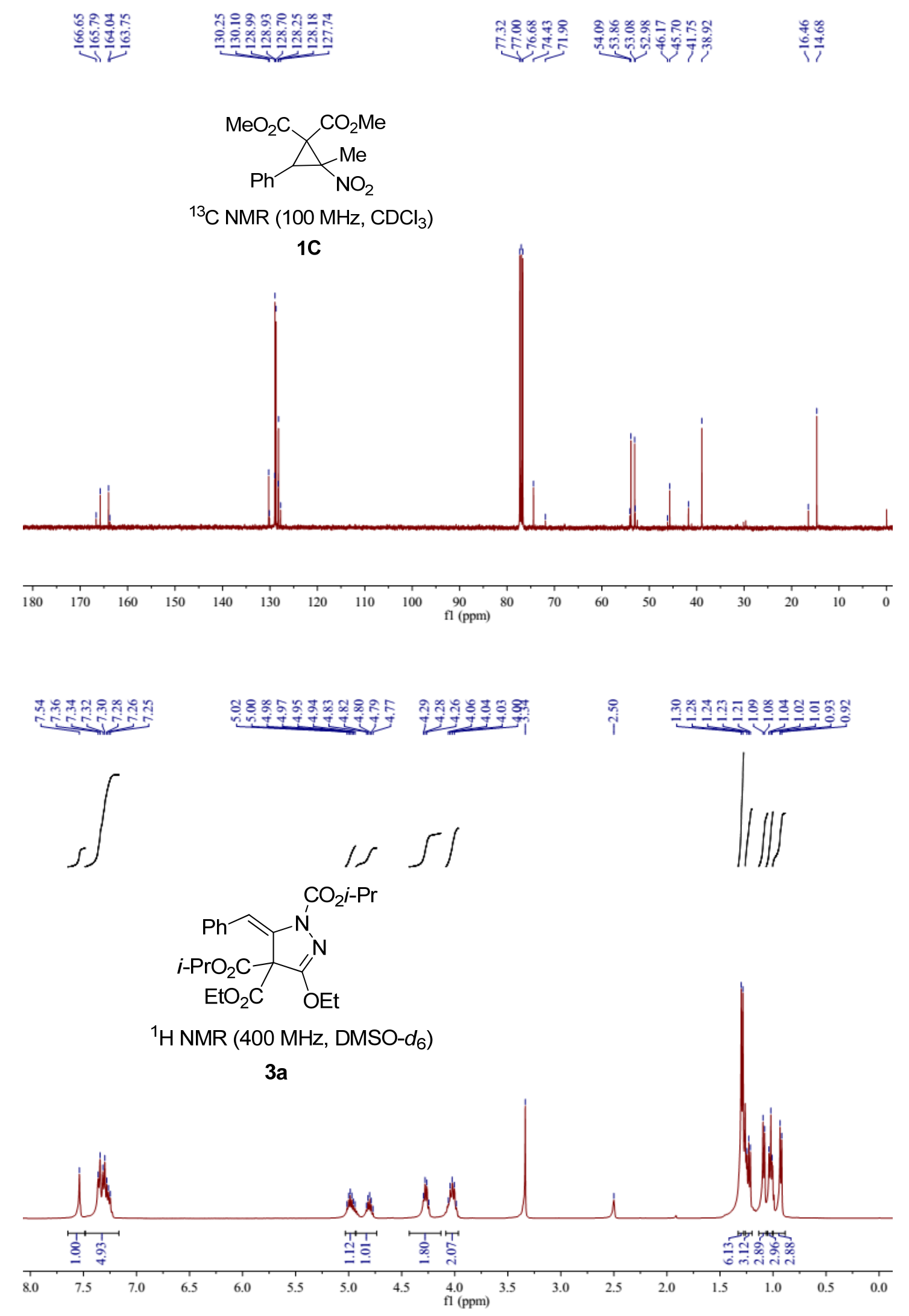




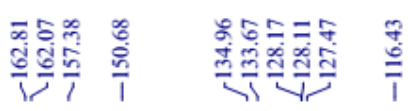

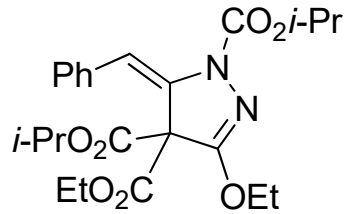

${ }^{13} \mathrm{C}$ NMR (100 MHz, DMSO- $d_{6}$ )

$3 a$
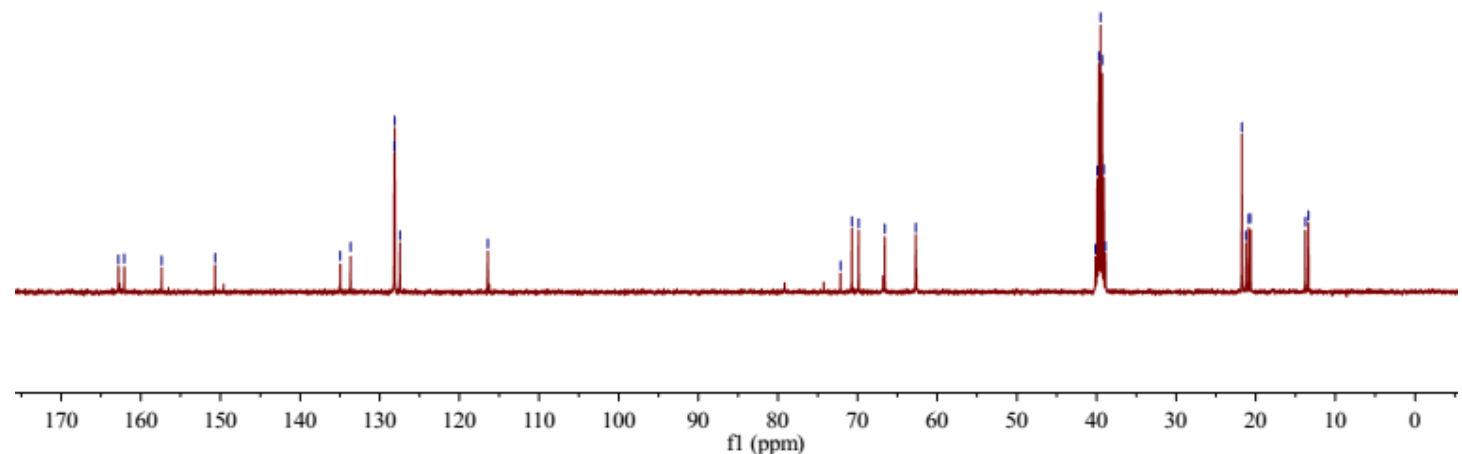

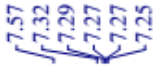

$\int$

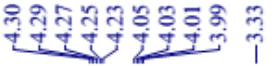

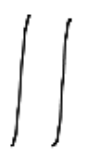

ํํำ

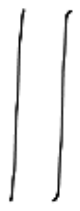<smiles>CCOC(=O)N1N=C(OCC)C(C)(C(=O)OCC)C1=Cc1ccccc1</smiles>

${ }^{1} \mathrm{H}$ NMR $\left(400 \mathrm{MHz}\right.$, DMSO- $\left.d_{6}\right)$

$3 b$

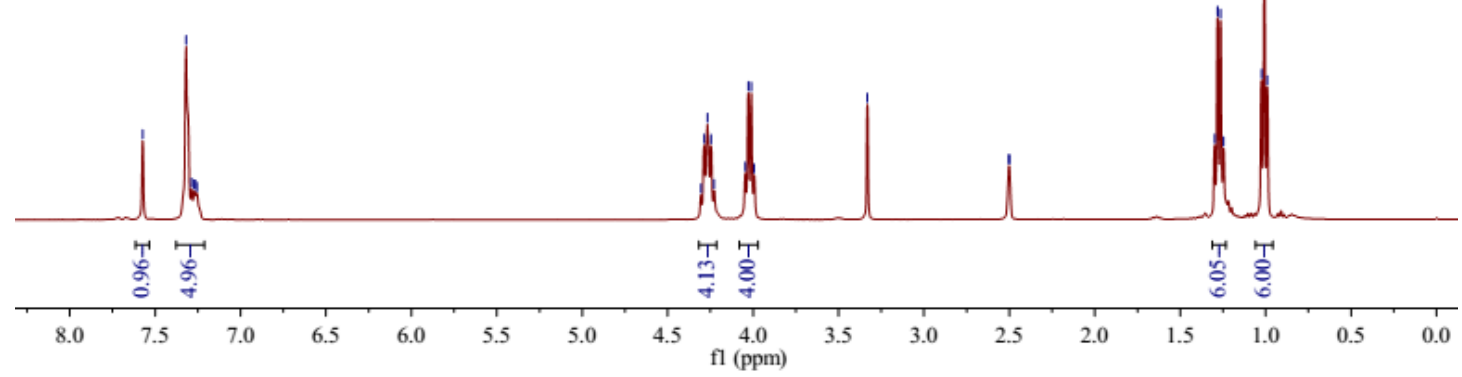




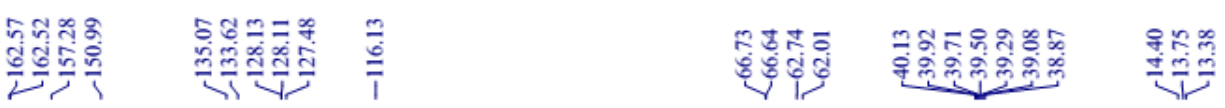

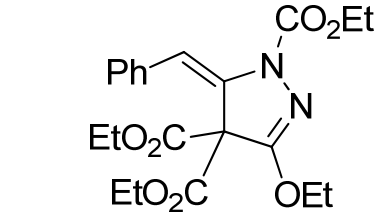

${ }^{13} \mathrm{C}$ NMR (100 MHz, DMSO- $d_{6}$ )

3b
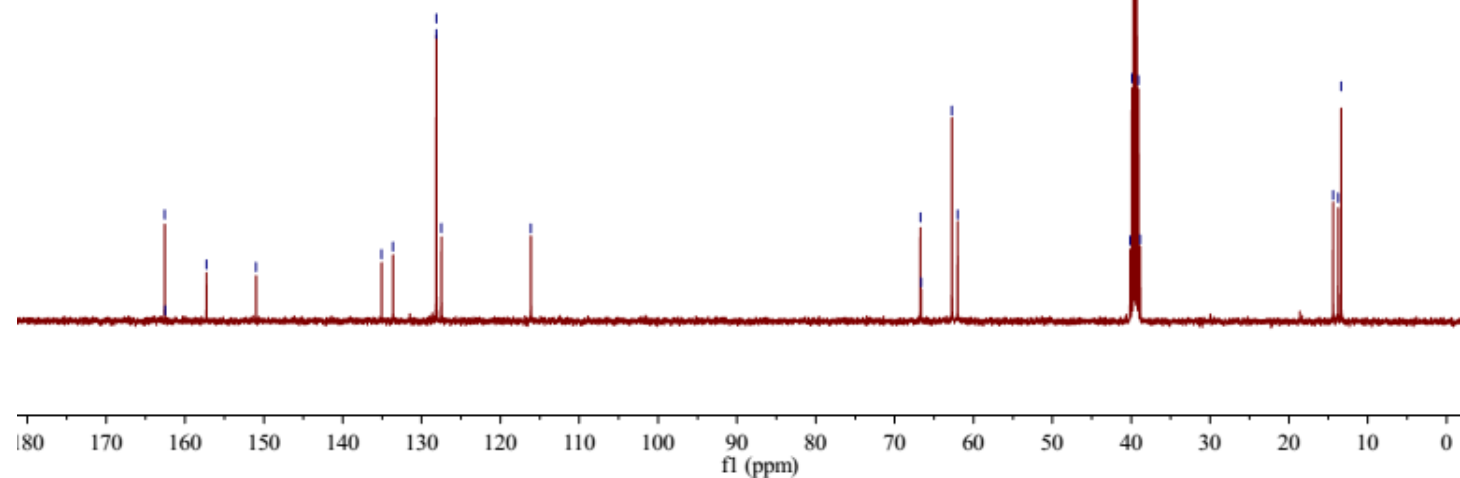

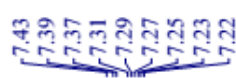

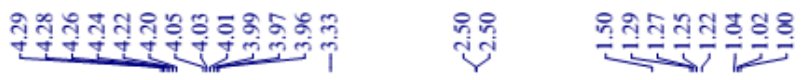

dlf

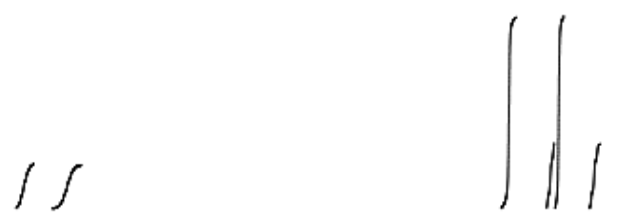<smiles>CCOC(=O)N1N=C(OCC)C(C)(C(=O)OCC)/C1=C\c1ccccc1</smiles>

${ }^{1} \mathrm{H}$ NMR (400 MHz, DMSO- $d_{6}$ )

3c

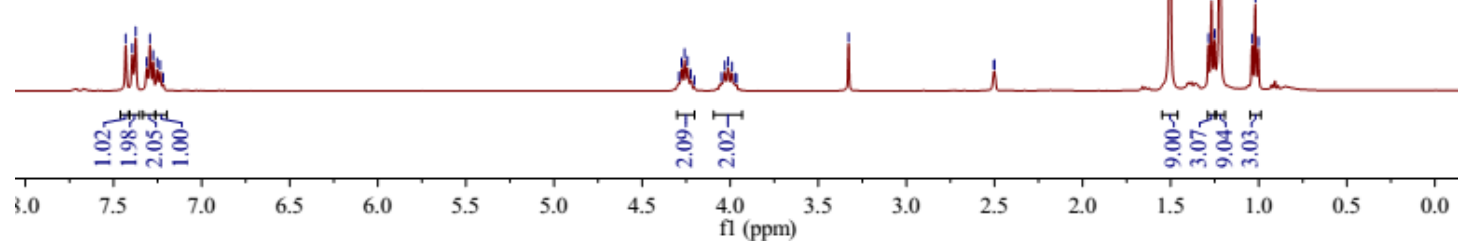




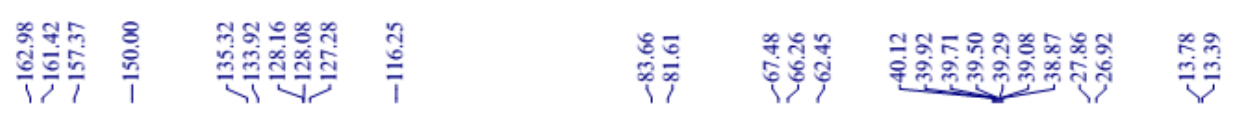

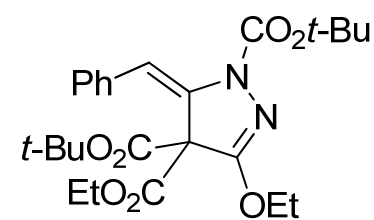

${ }^{13} \mathrm{C}$ NMR (100 MHz, DMSO- $d_{6}$ )

3c
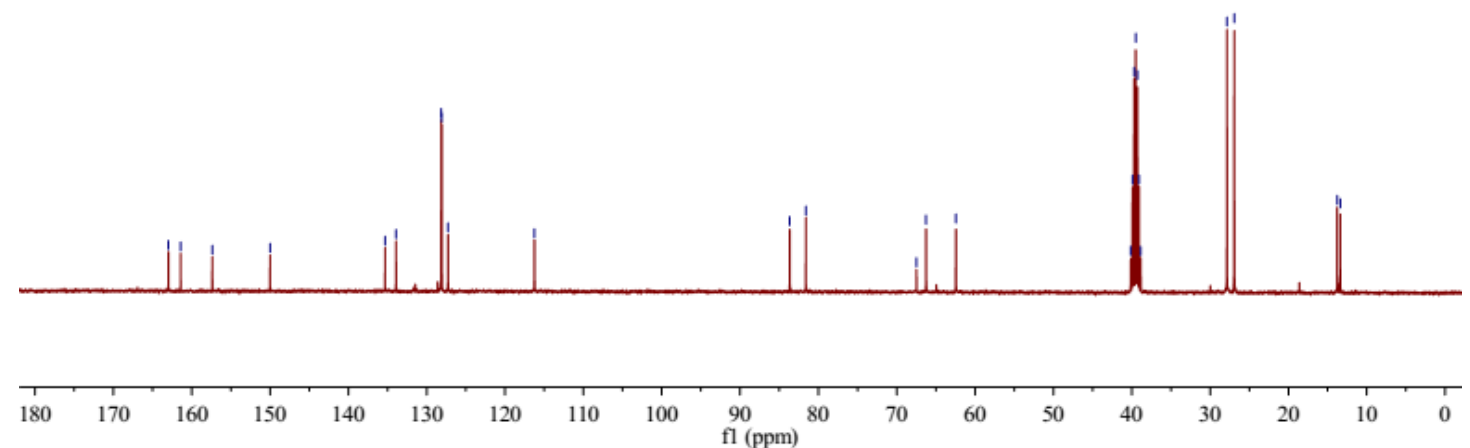

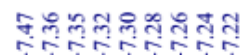

i

slls

$\iint$
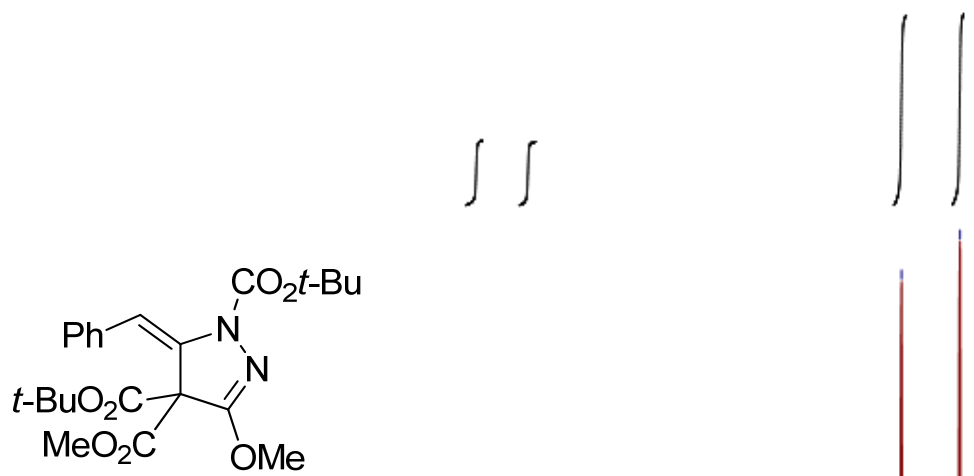

${ }^{1} \mathrm{H}$ NMR (400 MHz, DMSO- $\left.d_{6}\right)$

3d

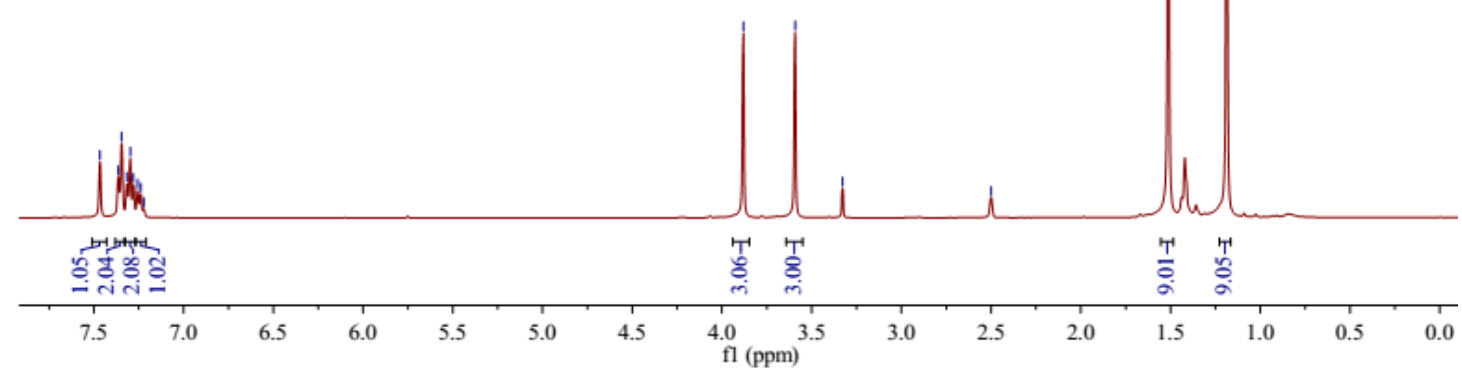




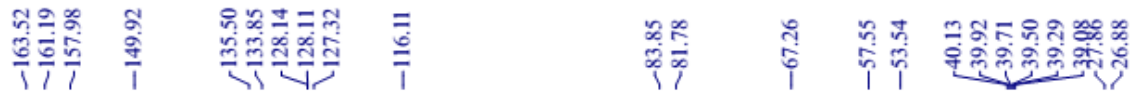

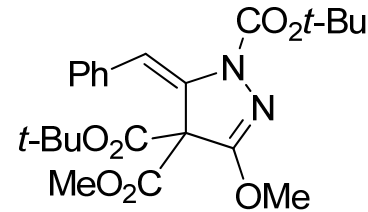

${ }^{13} \mathrm{C}$ NMR (100 MHz, DMSO- $d_{6}$ )

3d

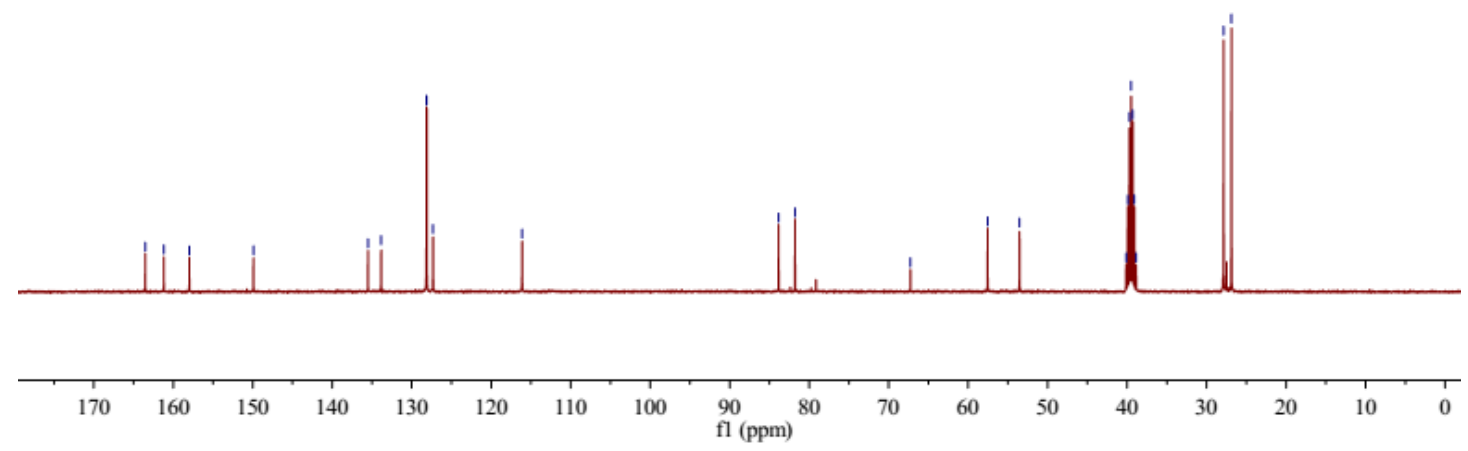

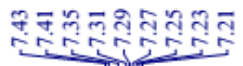

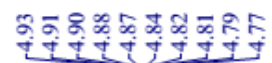

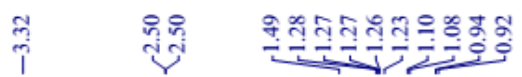

Ists

II<smiles>CCCOC(=O)N/N=C(/C=C\c1ccccc1)C(C)(C(=O)OCC)C(=O)OC(C)(C)C</smiles>

${ }^{1} \mathrm{H}$ NMR (400 MHz, DMSO- $d_{6}$ )

$3 e$

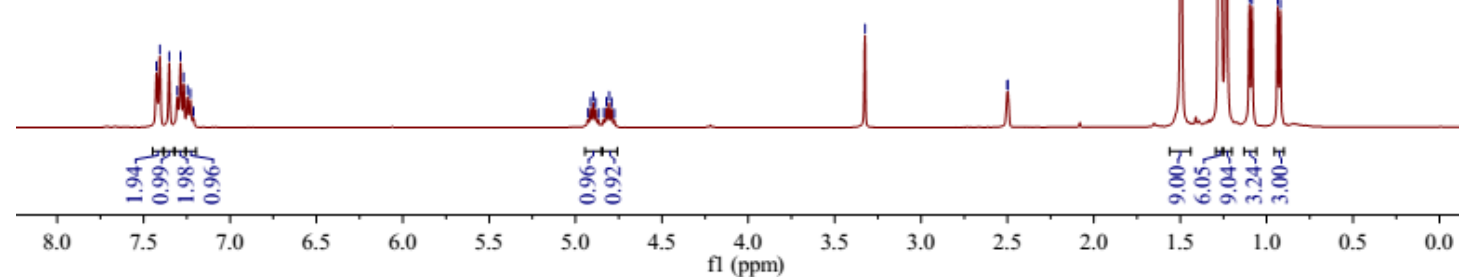




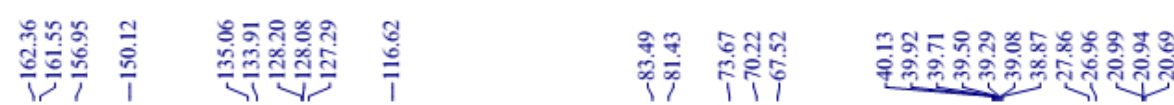

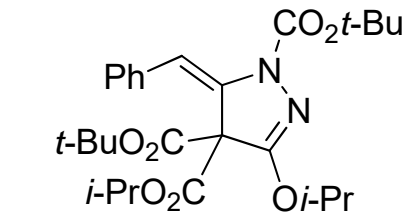

${ }^{13} \mathrm{C}$ NMR $\left(100 \mathrm{MHz}\right.$, DMSO- $\left.d_{6}\right)$

3 e
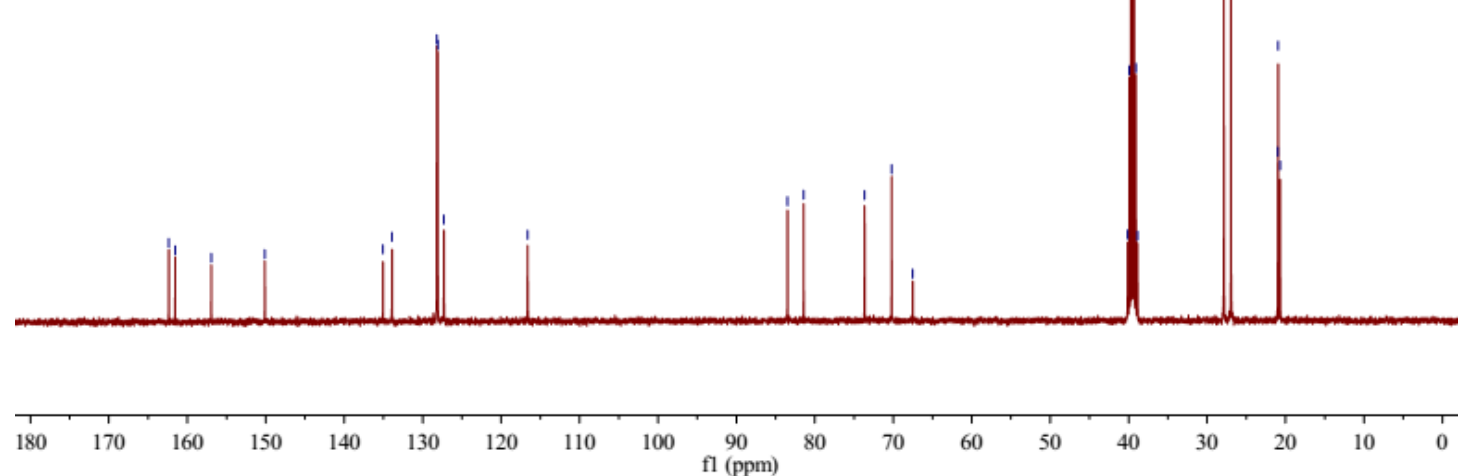

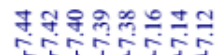

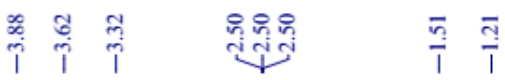

d1

$\iint$

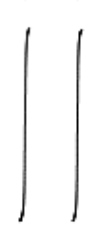<smiles>COC(=O)C1(C)C(OC)=NN(C(=O)OC(C)(C)C)/C1=C/c1ccc(F)c([18O])c1</smiles>

${ }^{1} \mathrm{H}$ NMR (400 MHz, DMSO- $d_{6}$ )

$3 f$

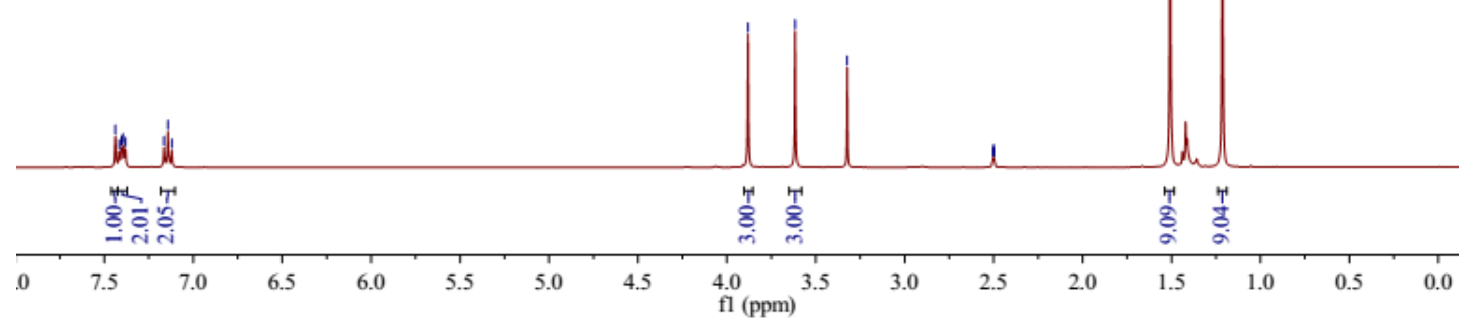




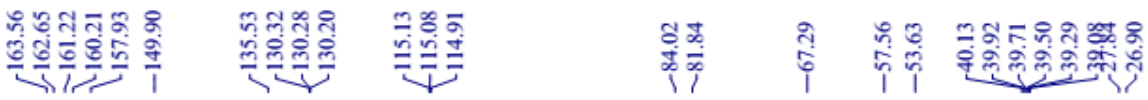

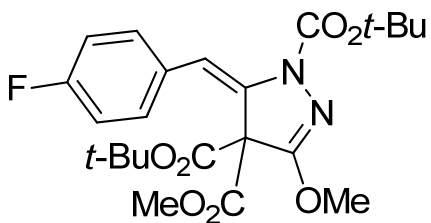

${ }^{13} \mathrm{C}$ NMR (100 MHz, DMSO- $d_{6}$ )

$3 f$
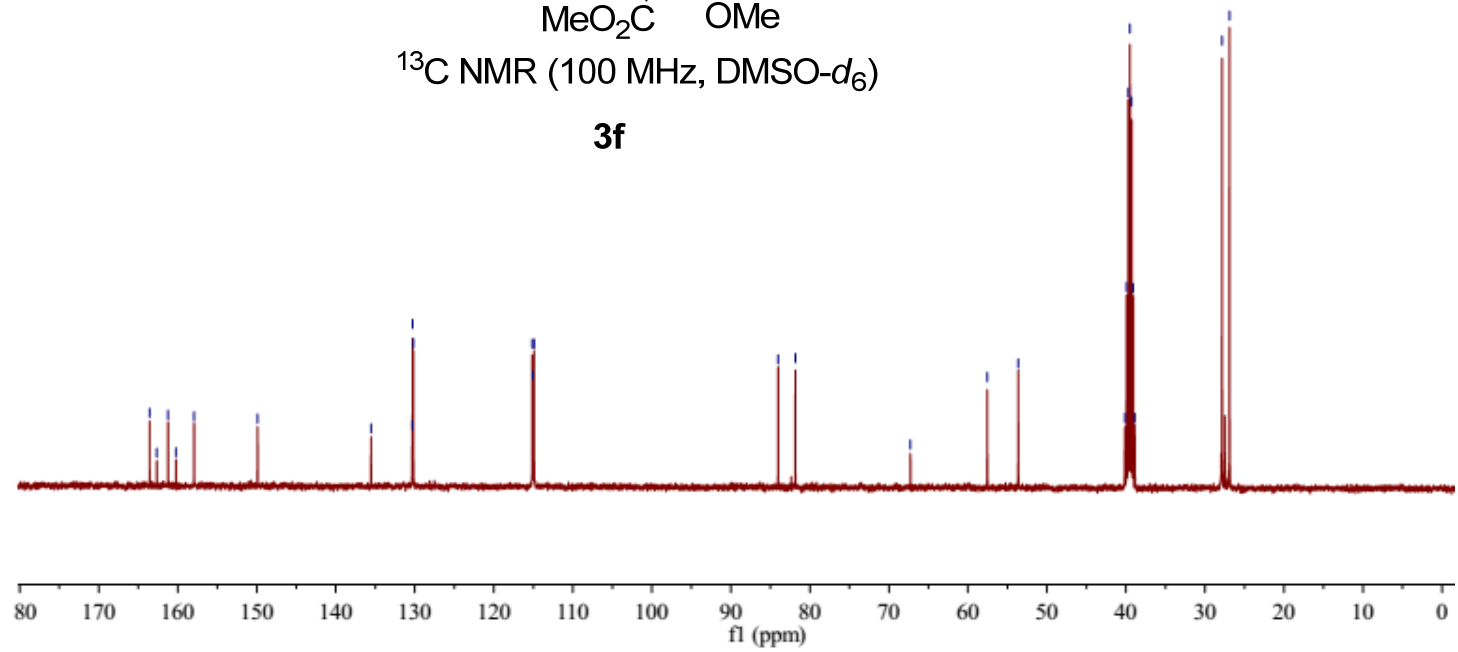

$\stackrel{\text { ก }}{\stackrel{\text { i }}{i}}$

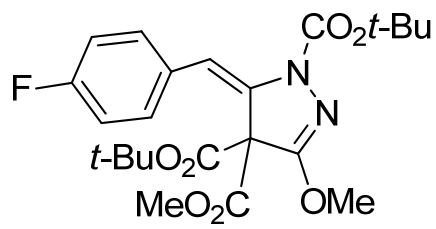

${ }^{19}$ F NMR (376 MHz, DMSO-d ${ }_{6}$ )

$3 f$

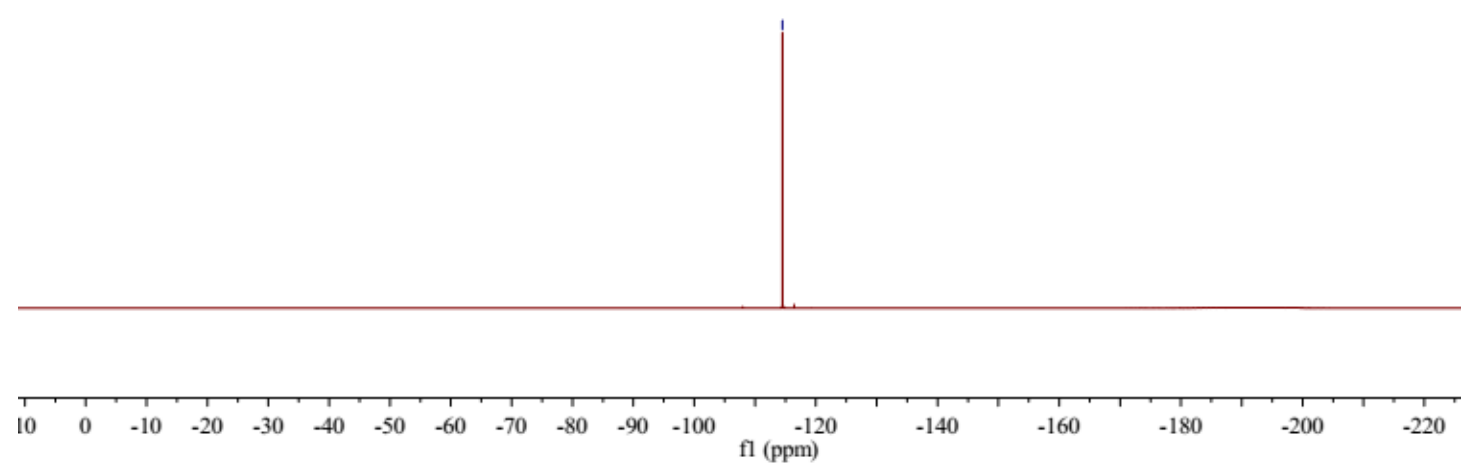


11<smiles>COC(=O)C1(C(=O)OC)C(=Cc2ccccc2Cl)N(C(=O)OC(C)(C)C)N=C1OC(C)(C)C</smiles>

${ }^{1} \mathrm{H}$ NMR (400 MHz, DMSO- $d_{6}$ )

$3 g$

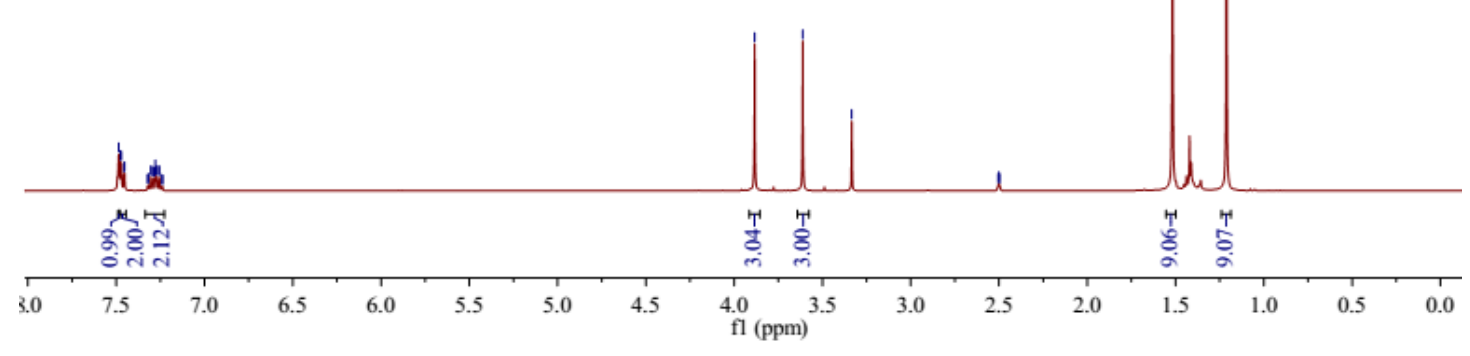

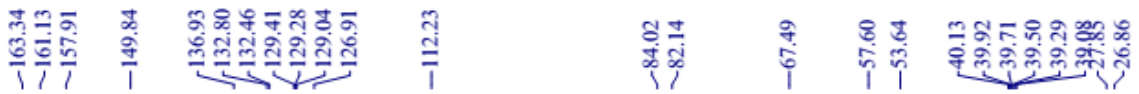

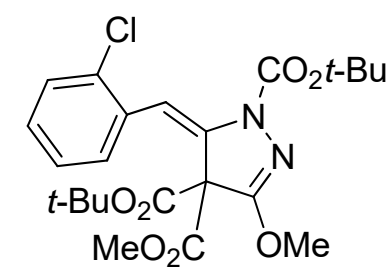

${ }^{13} \mathrm{C}$ NMR (100 MHz, DMSO- $d_{6}$ )

3g
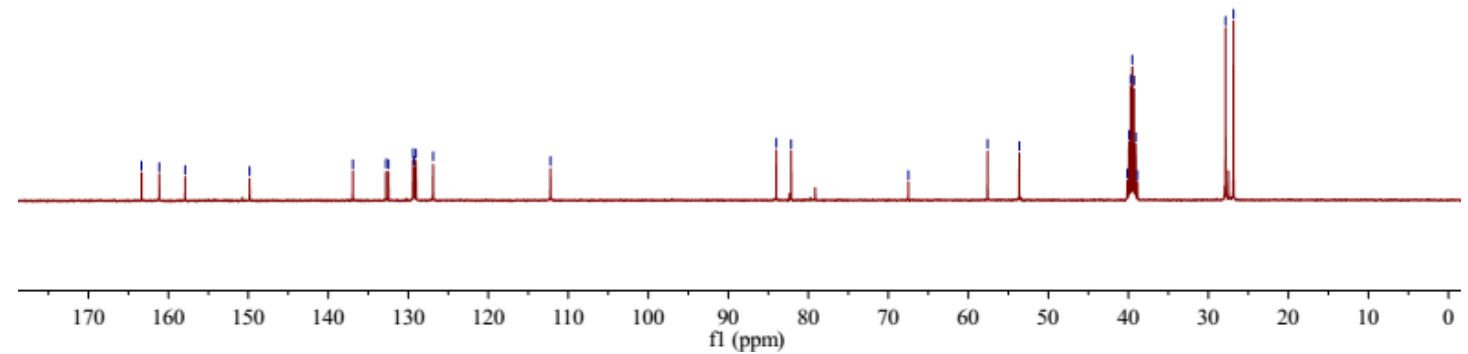
II

$\iint$

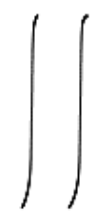<smiles>COC(=O)C1(C(=O)OC)C(=Cc2ccccc2)N(C(=O)OC(C)(C)C)C1=Cc1cc([13CH3])cc(Cl)c1</smiles>

${ }^{1} \mathrm{H}$ NMR $\left(400 \mathrm{MHz}\right.$, DMSO- $\left.d_{6}\right)$

3h

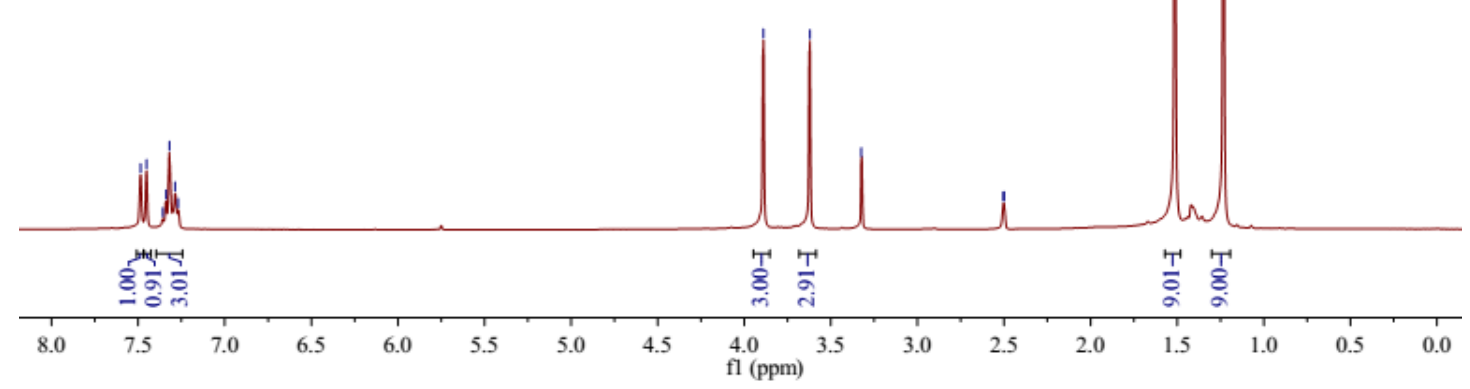

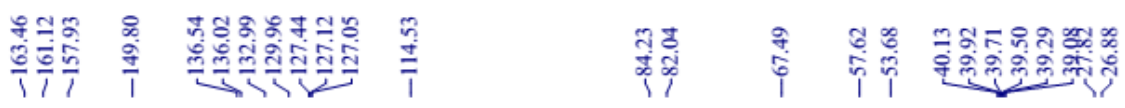

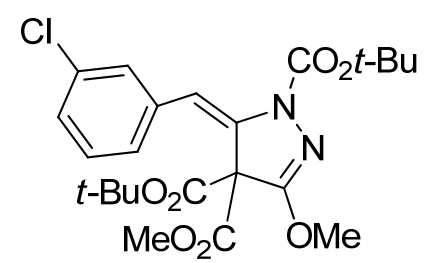

${ }^{13} \mathrm{C}$ NMR (100 MHz, DMSO- $\left.d_{6}\right)$

$3 \mathbf{h}$

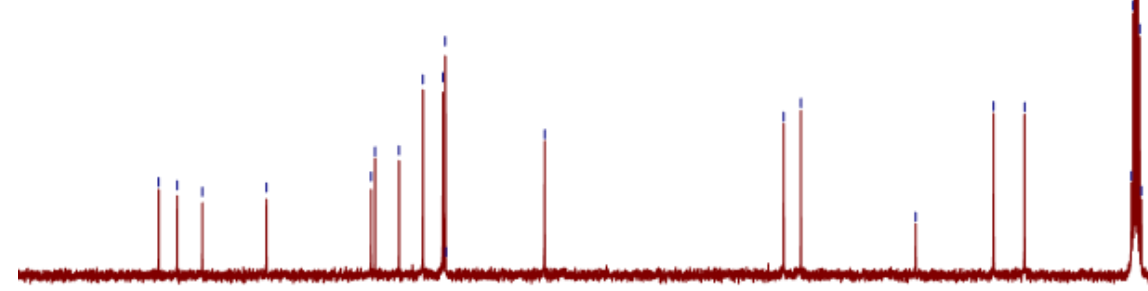

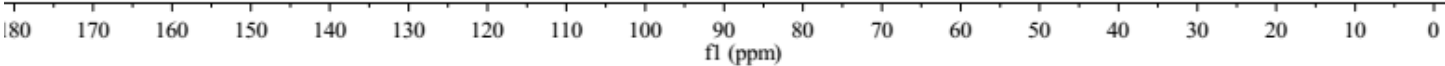



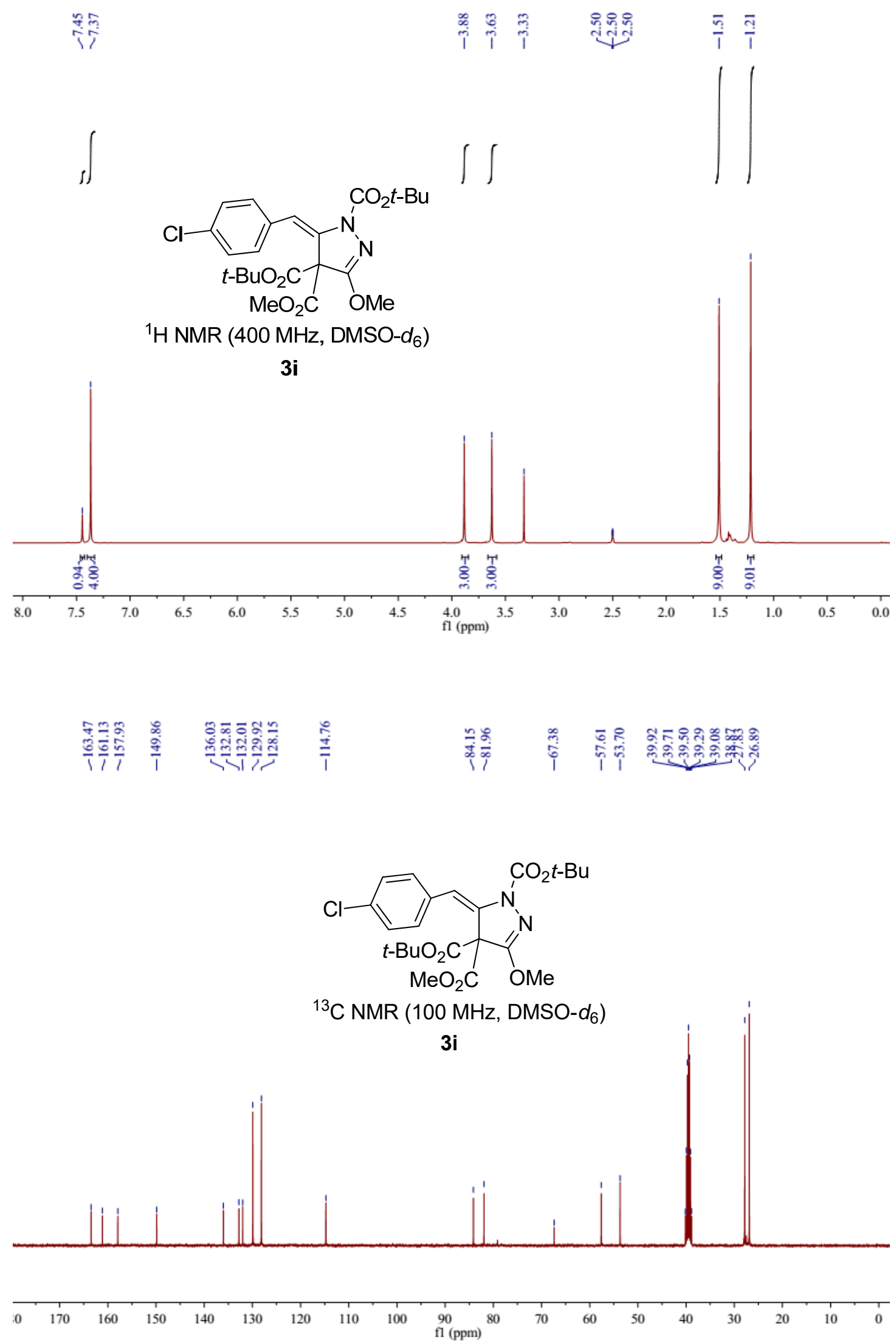


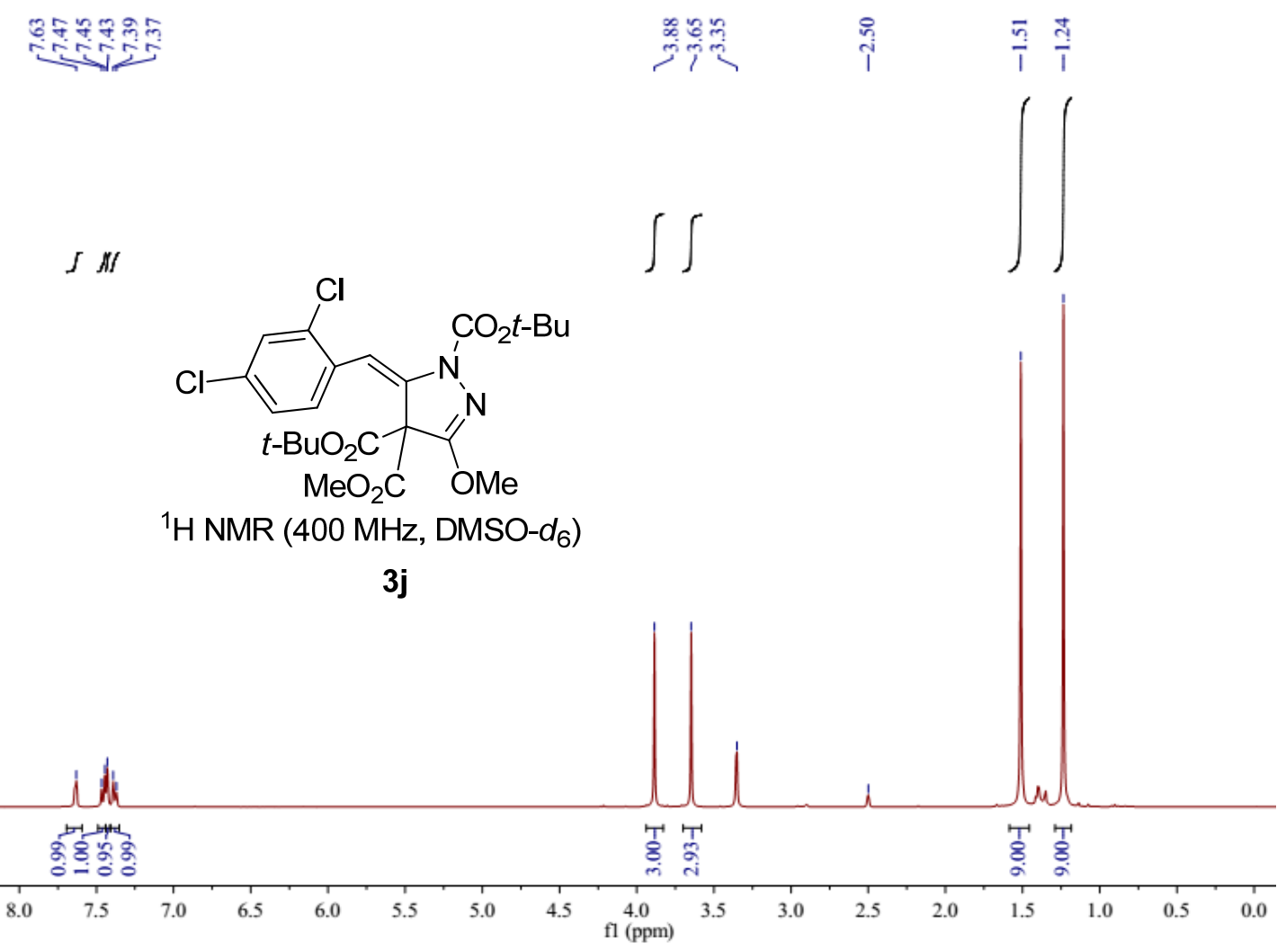

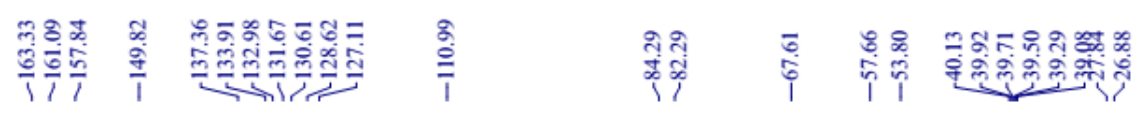

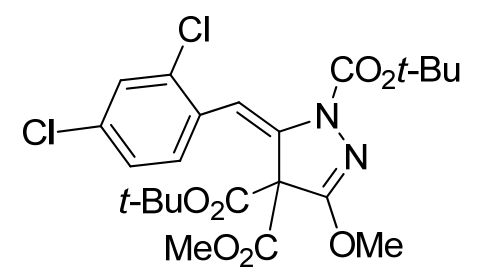

${ }^{13} \mathrm{C}$ NMR $\left(100 \mathrm{MHz}\right.$, DMSO- $\left.d_{6}\right)$

3j

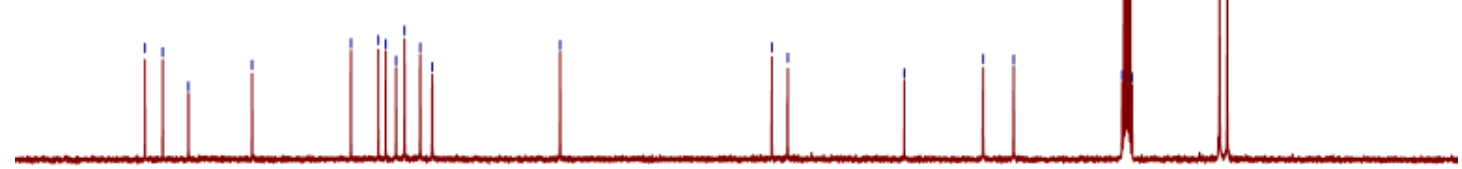

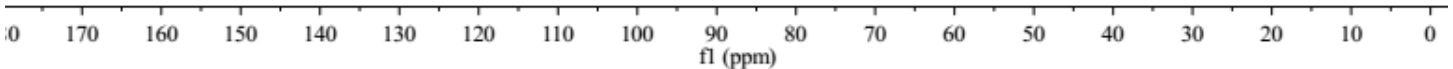


$I / / /$

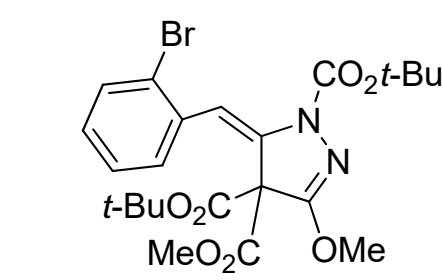

${ }^{1} \mathrm{H}$ NMR (400 MHz, DMSO- $d_{6}$ )

3k

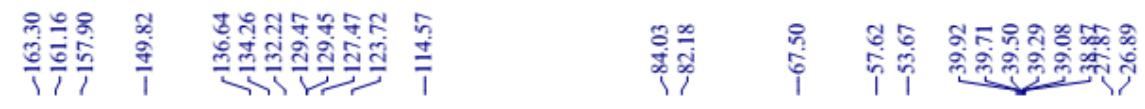

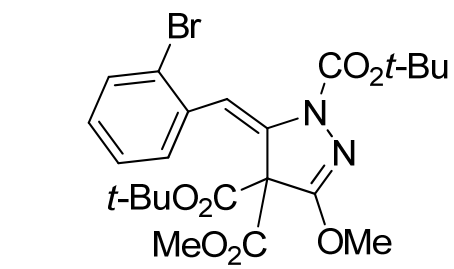

${ }^{13} \mathrm{C}$ NMR (100 MHz, DMSO-d $)$

3k

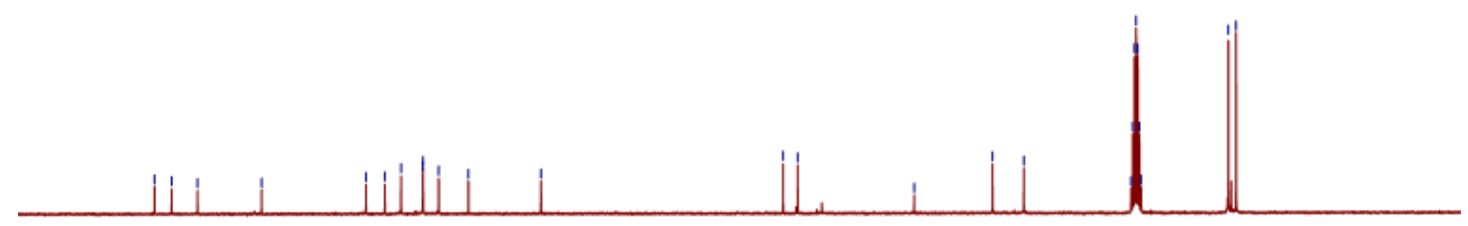

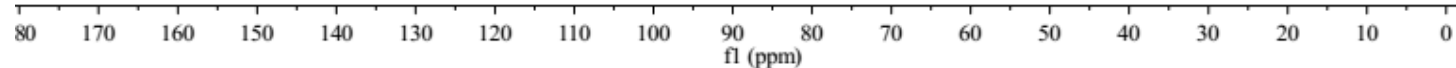


I $H$<smiles>COC(=O)OC(=O)N1N=C(OC)C(C(=O)OC)(C(=O)OC)C1=Cc1ccc(Br)cc1</smiles>

$\iint$

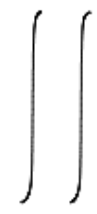

${ }^{1} \mathrm{H}$ NMR (400 MHz, DMSO- $d_{6}$ )

3I

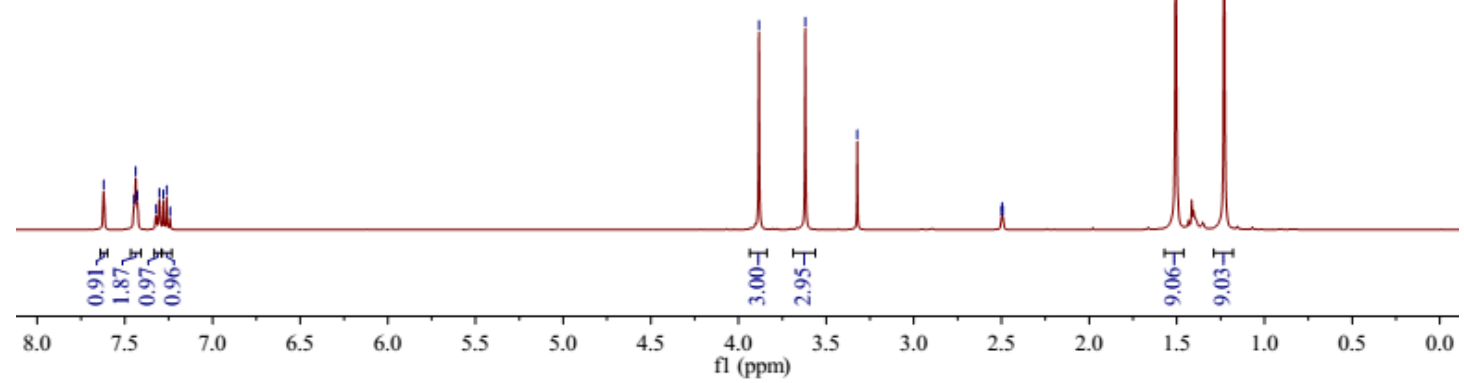

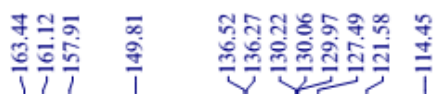

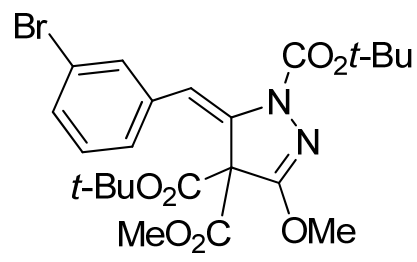

${ }^{13} \mathrm{C}$ NMR (100 MHz, DMSO- $d_{6}$ )

3I
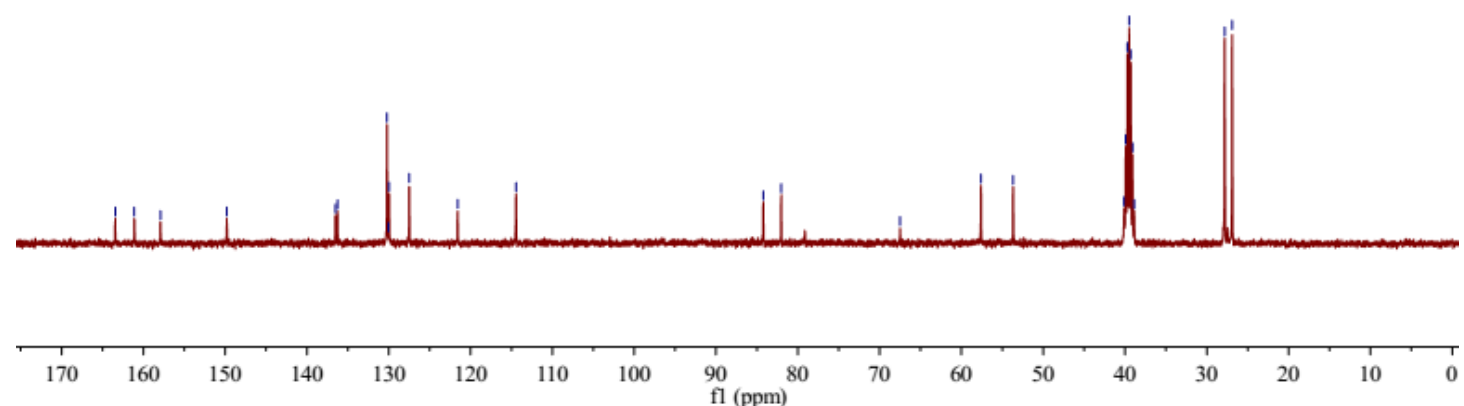


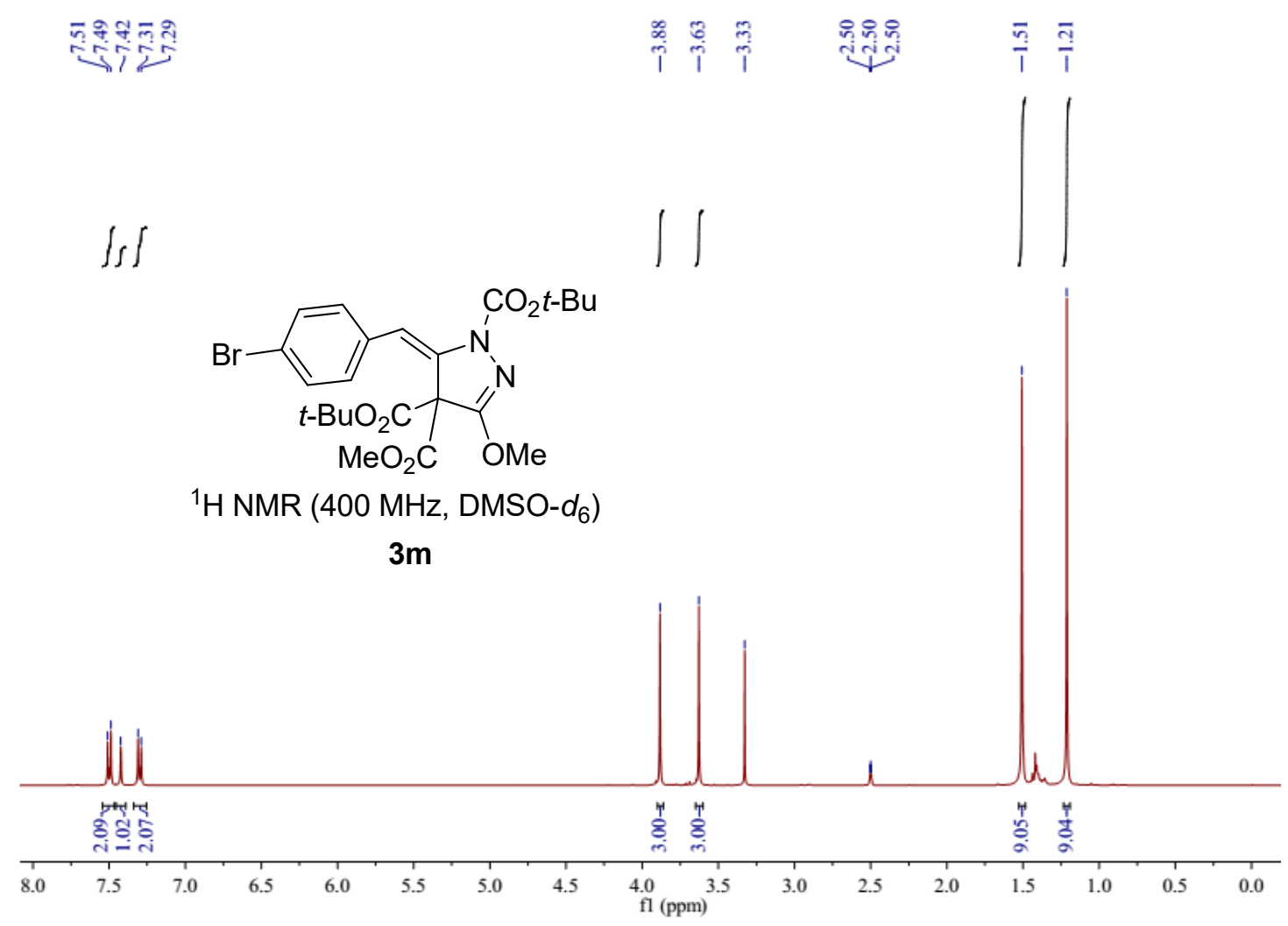

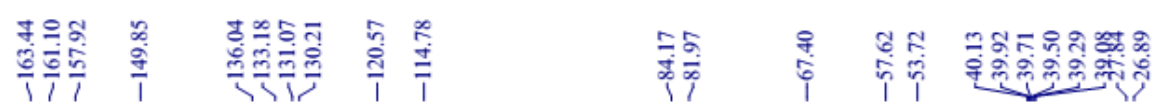

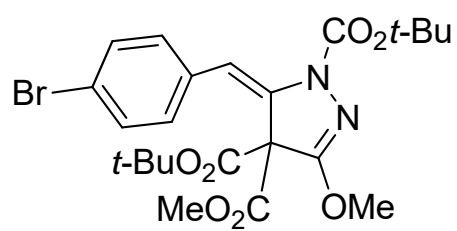

${ }^{13} \mathrm{C}$ NMR (100 MHz, DMSO-d $)$

$3 \mathrm{~m}$

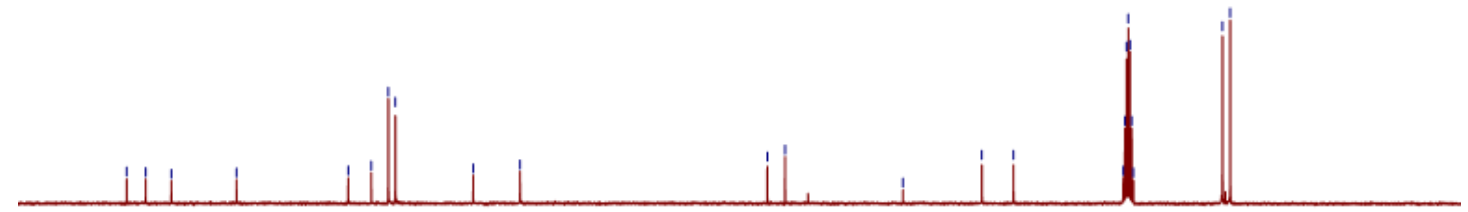

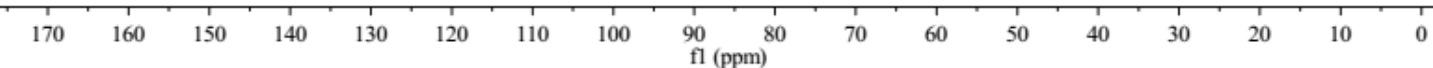


$\iint /$<smiles>COC(=O)C1(C(=O)OC)C(=Cc2ccccc2C)N(C(=O)OC(C)(C)C)N=C1OC</smiles><smiles>CCCC</smiles>

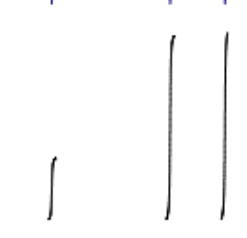

${ }^{1} \mathrm{H}$ NMR (400 MHz, DMSO- $\left.d_{6}\right)$

3n
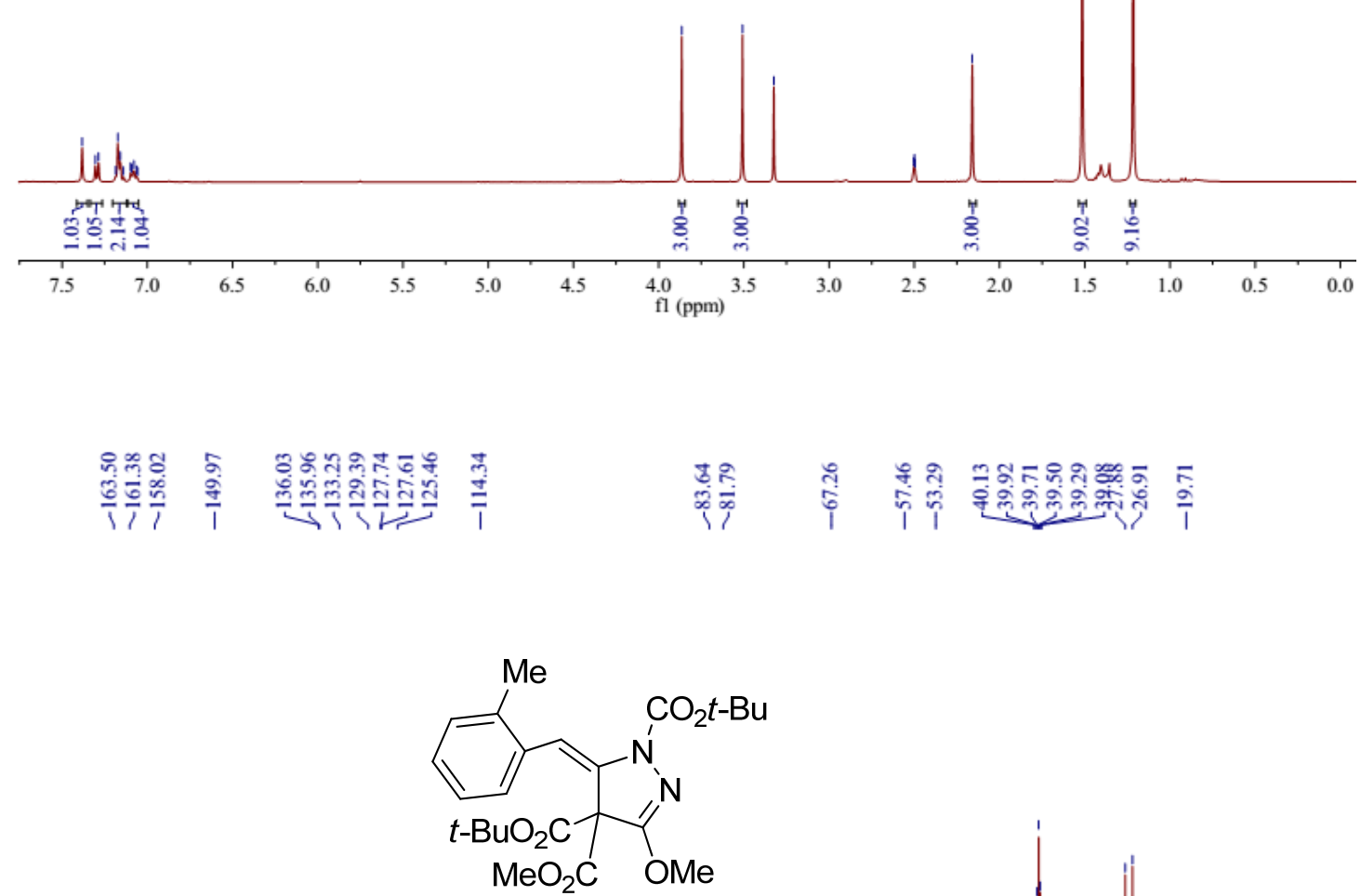

${ }^{13} \mathrm{C}$ NMR (100 MHz, DMSO- $\left.d_{6}\right)$

$3 n$

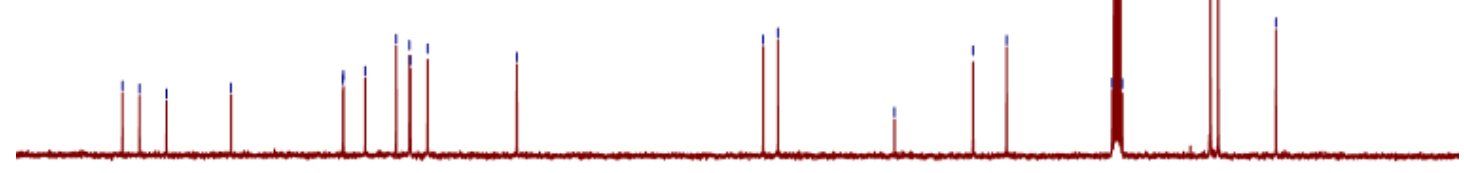

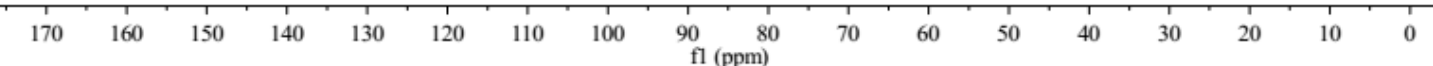




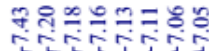

$s$ IfI

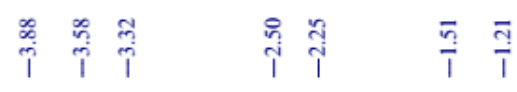

$\iint$

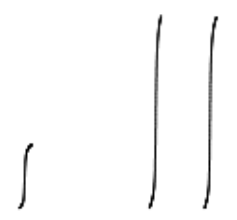

${ }^{1} \mathrm{H}$ NMR (400 MHz, DMSO- $\left.d_{6}\right)$

30

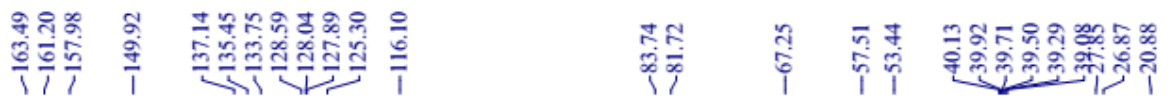

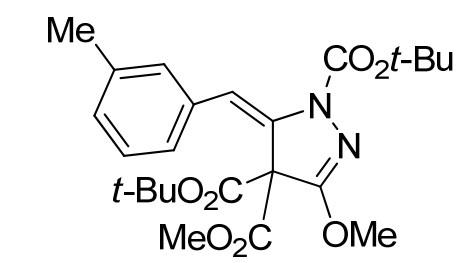

${ }^{13} \mathrm{C}$ NMR (100 MHz, DMSO- $\left.d_{6}\right)$

30

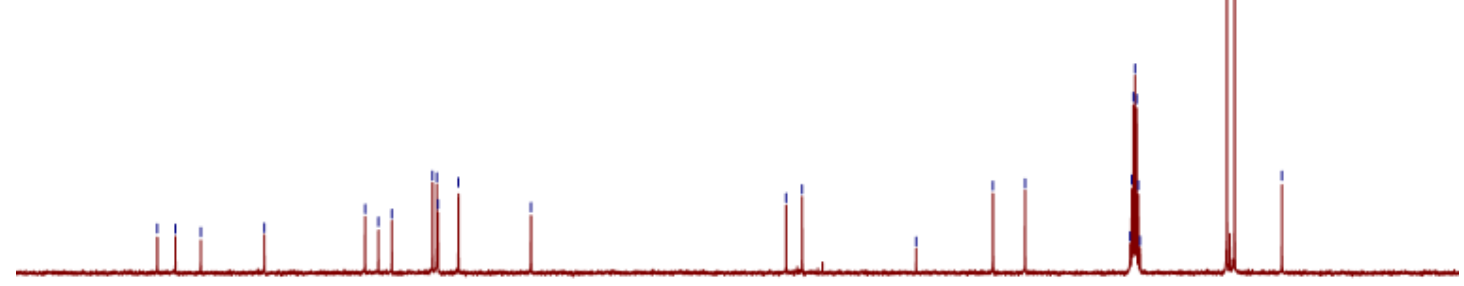

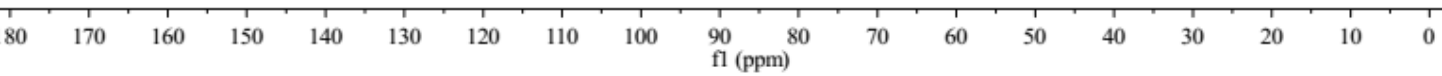




$$
\begin{aligned}
& \text { จำำ }
\end{aligned}
$$

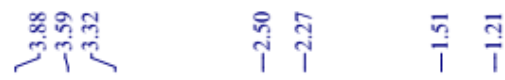

$$
\begin{aligned}
& \iint \\
& \iint
\end{aligned}
$$

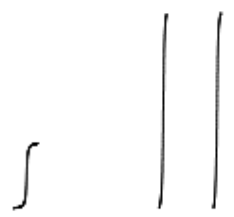

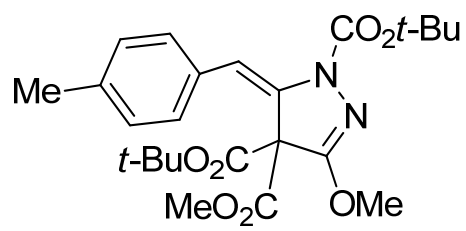

${ }^{1} \mathrm{H}$ NMR (400 MHz, DMSO- $d_{6}$ )

$3 p$

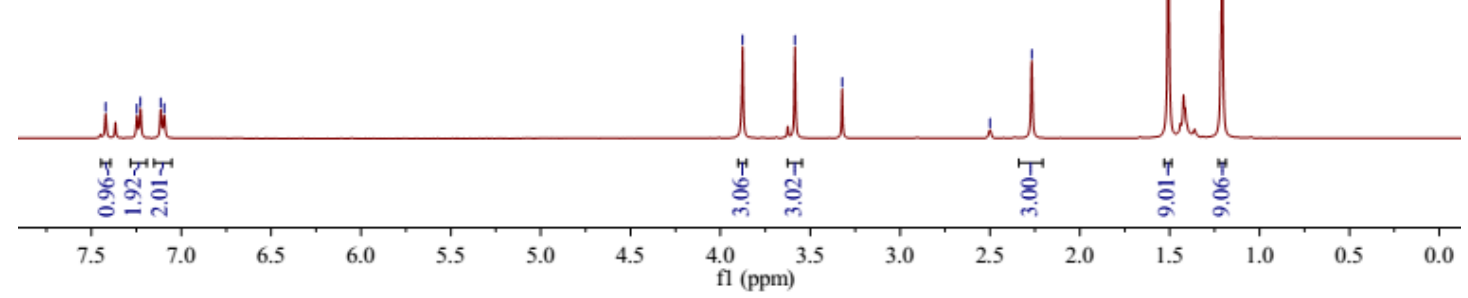

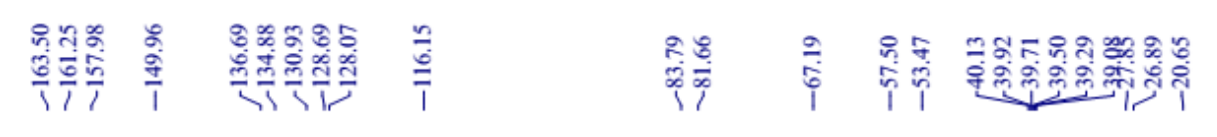

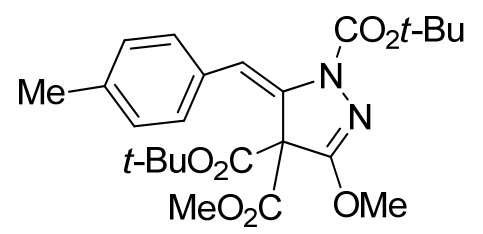

${ }^{13} \mathrm{C}$ NMR $\left(100 \mathrm{MHz}\right.$, DMSO- $\left.d_{6}\right)$

$3 p$

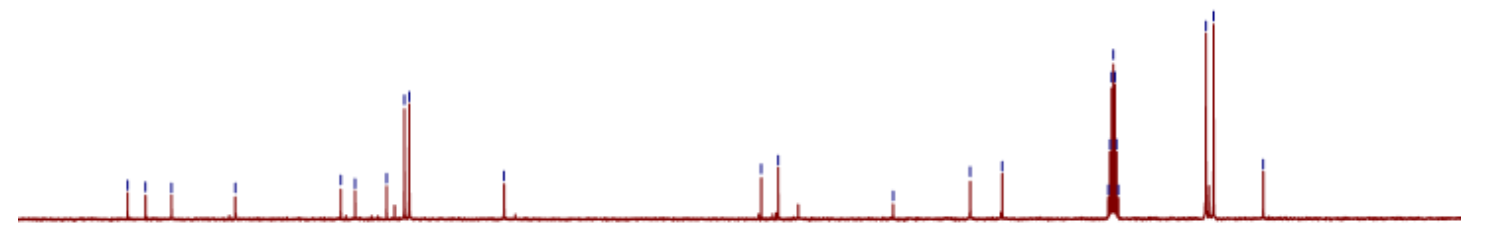

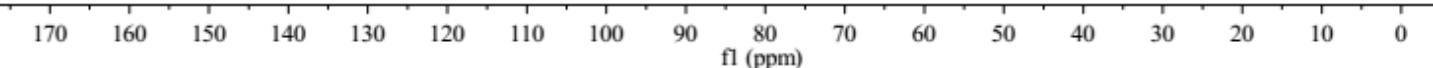




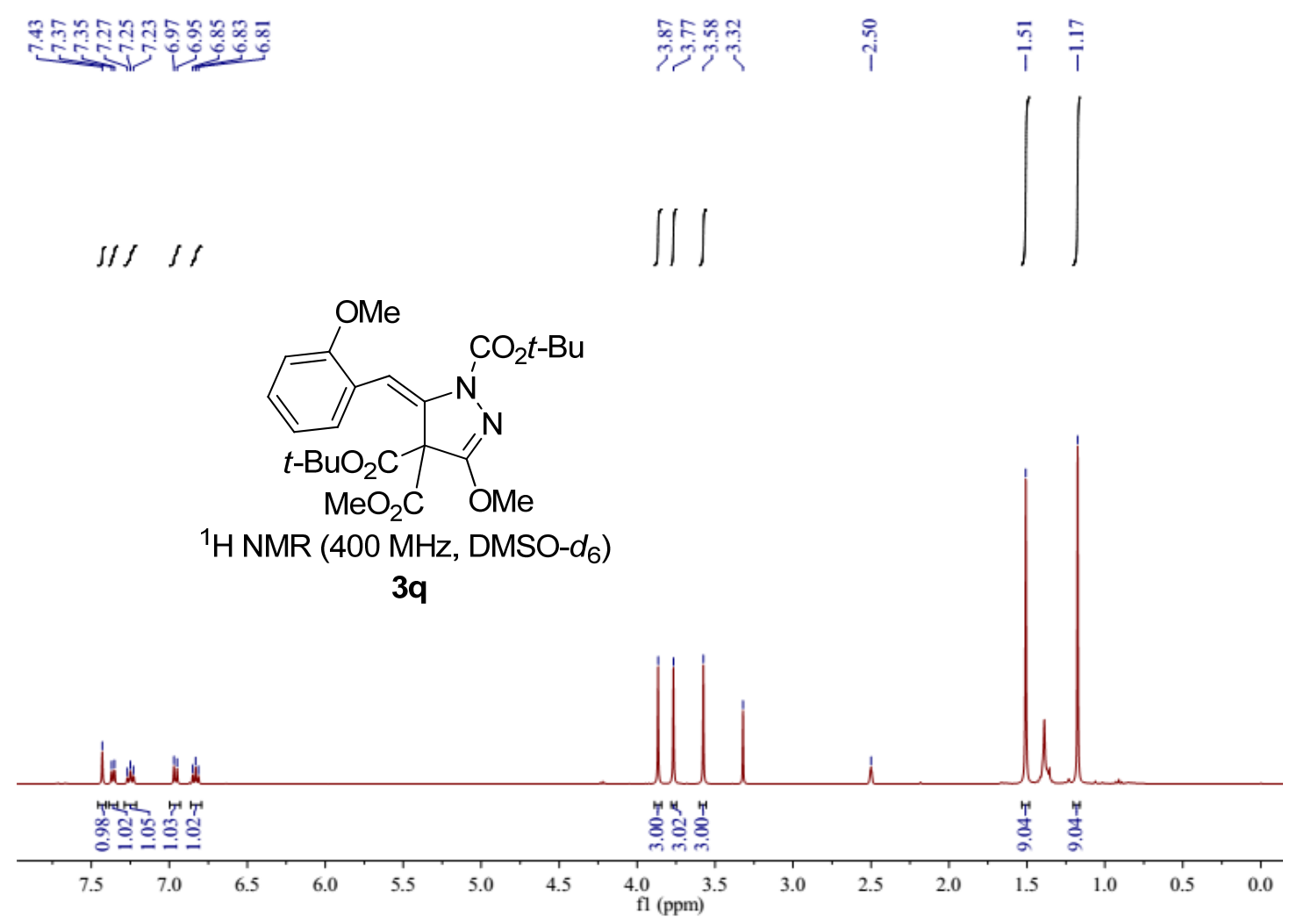

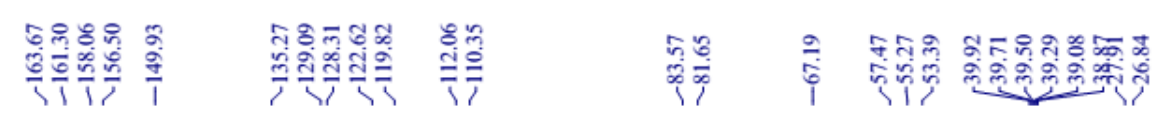

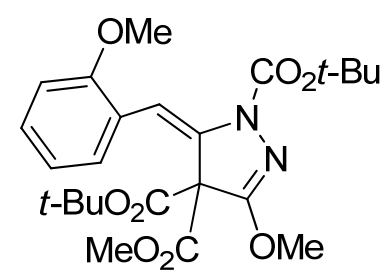

${ }^{13} \mathrm{C}$ NMR (100 MHz, DMSO- $\left.d_{6}\right)$

$3 q$

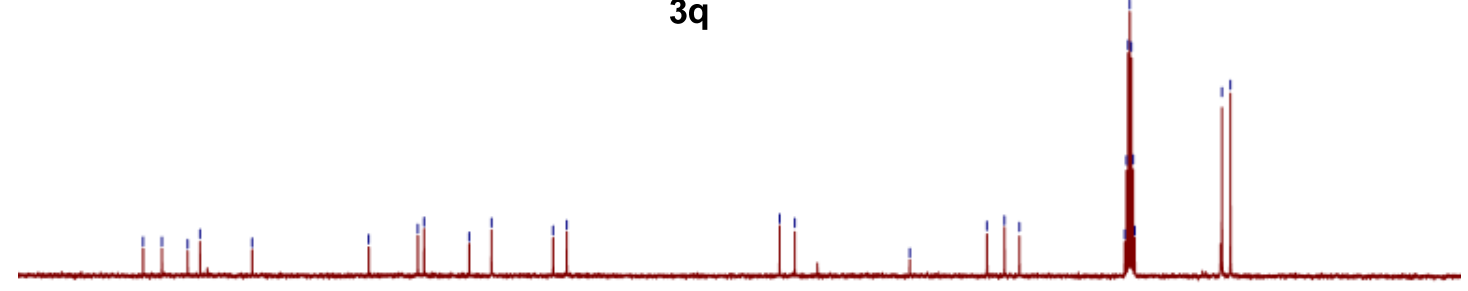

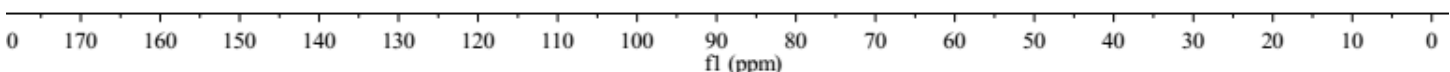




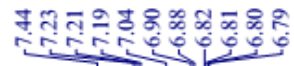

$2 \pi 2 \pi$

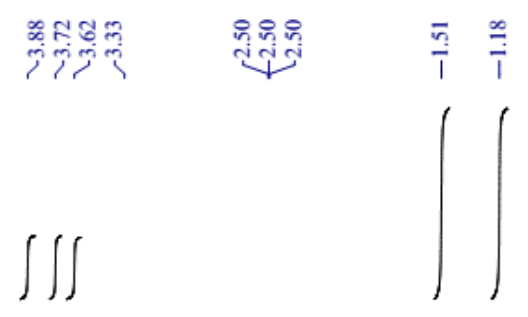

$\mathrm{MeO}$<smiles>COC(=O)C1(C)C(OC)=NN(C(=O)OC(C)(C)C)/C1=C\c1cccc(F)c1</smiles>

${ }^{1} \mathrm{H}$ NMR $\left(400 \mathrm{MHz}\right.$, DMSO- $\left.d_{6}\right)$

$3 r$

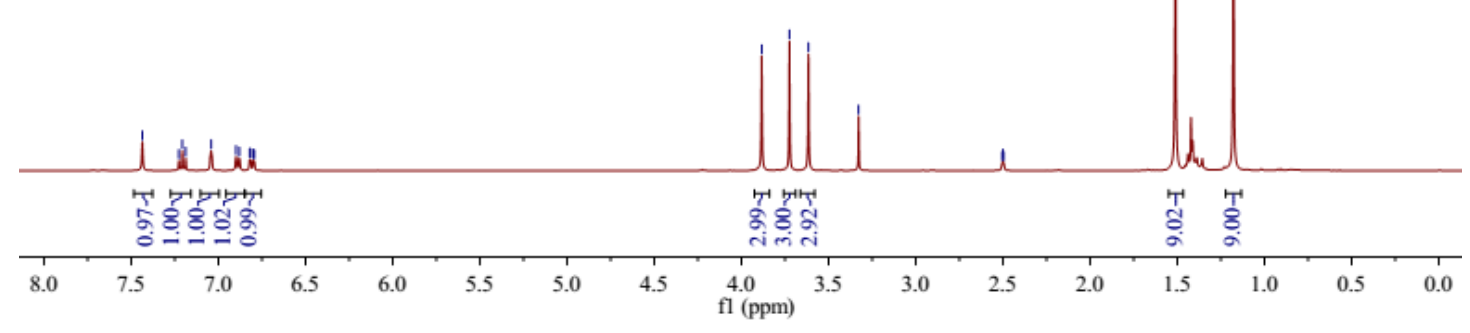

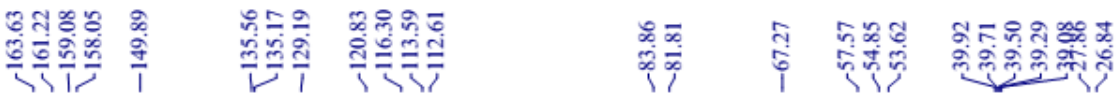

$\mathrm{MeO}$

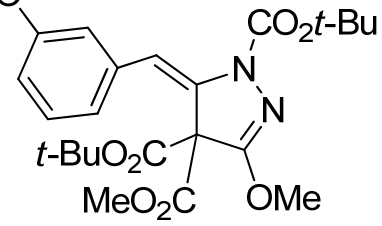

${ }^{13} \mathrm{C}$ NMR $\left(100 \mathrm{MHz}\right.$, DMSO- $\left.d_{6}\right)$

$3 r$

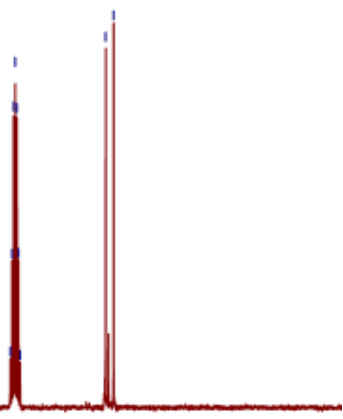

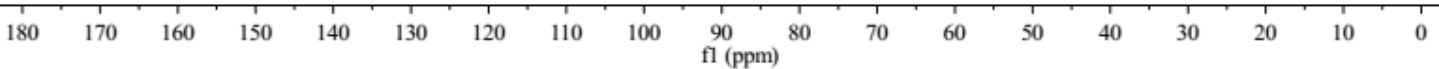



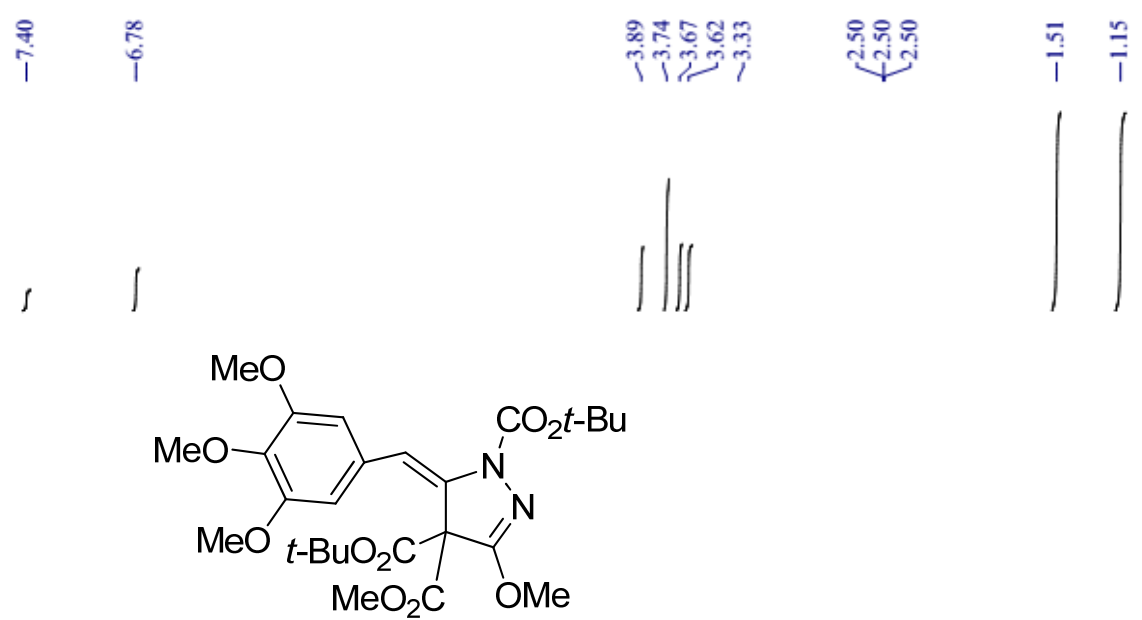

${ }^{1} \mathrm{H}$ NMR (400 MHz, DMSO- $d_{6}$ )

3s

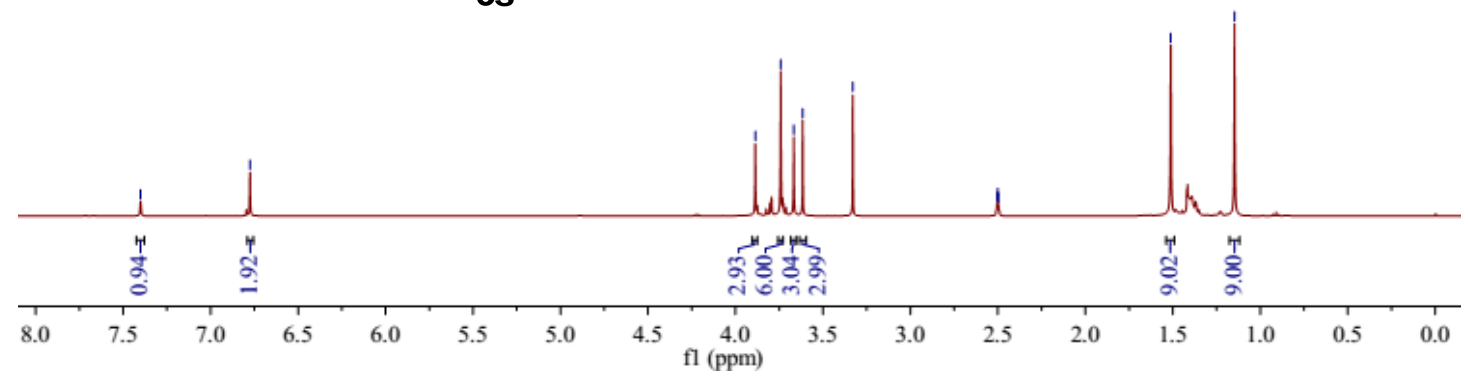

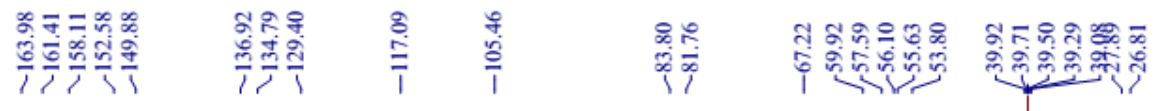<smiles>COCC1(C)C(=Cc2cc(OC)c(OC)c(OC)c2)N(C(=O)OC(=O)OC)N=C1OC</smiles>

${ }^{13} \mathrm{C}$ NMR (100 MHz, DMSO- $d_{6}$ )

3s

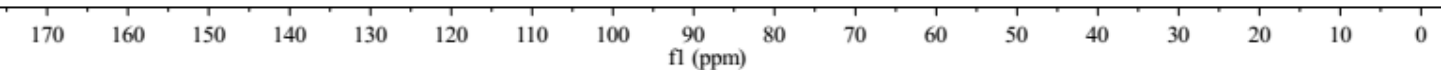


$1 / 1 / 1$

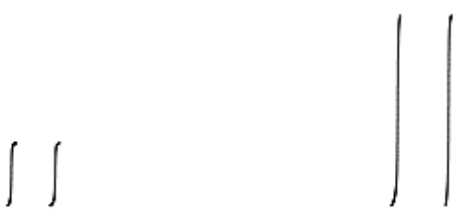<smiles>COC(=O)C1(C(=O)OC)C(=Cc2cccc([N+](=O)[O-])c2)N(C(=O)OC(C)(C)C)N=C1OC</smiles>

${ }^{1} \mathrm{H}$ NMR $\left(400 \mathrm{MHz}\right.$, DMSO- $\left.d_{6}\right)$

$3 \mathbf{t}$
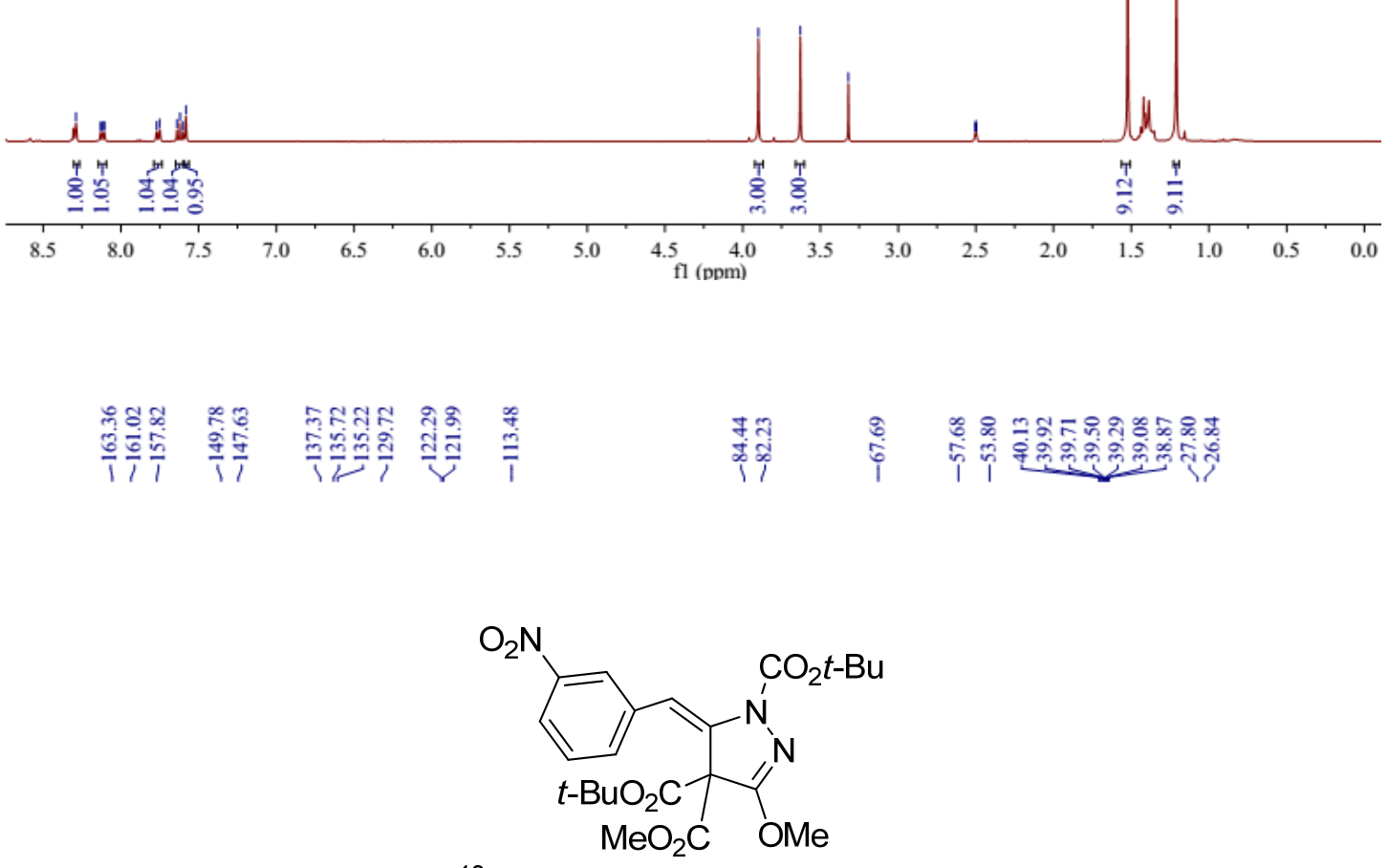

${ }^{13} \mathrm{C}$ NMR (100 MHz, DMSO- $d_{6}$ )

3t
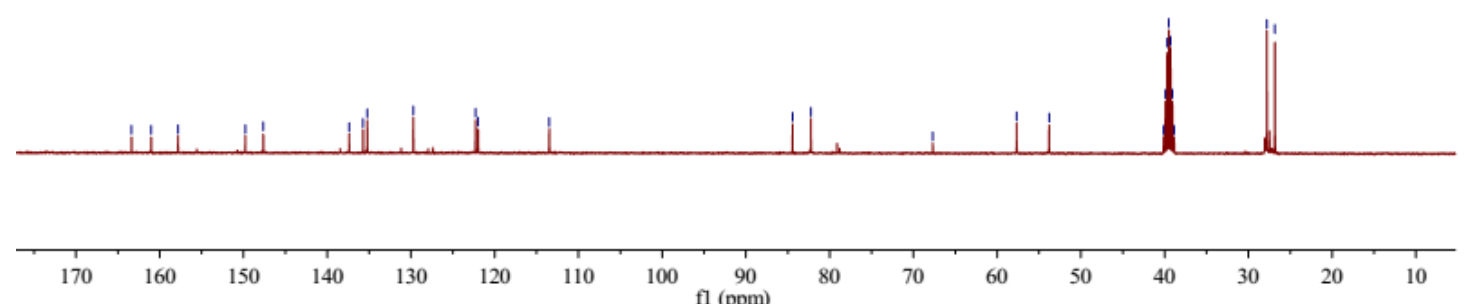


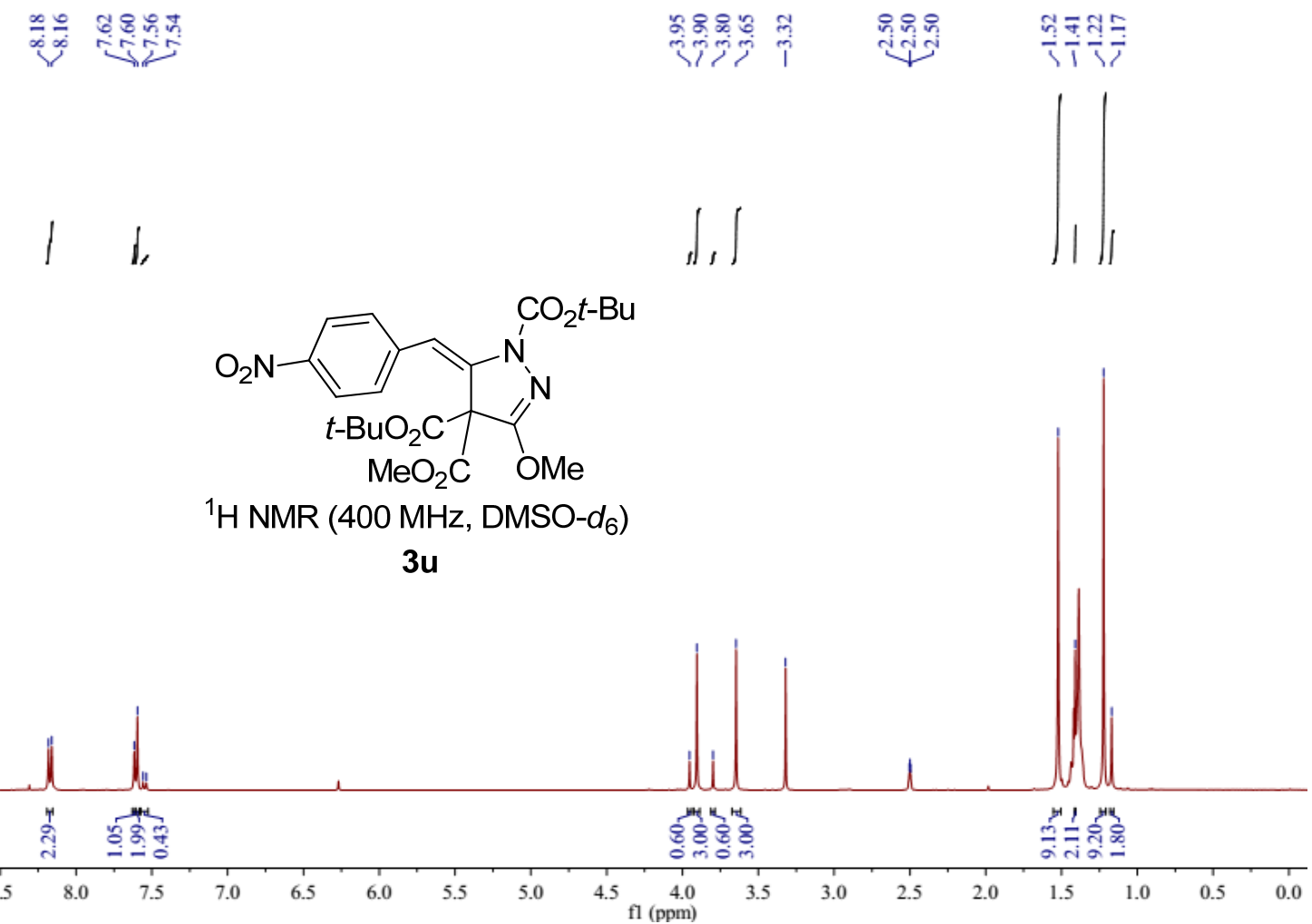

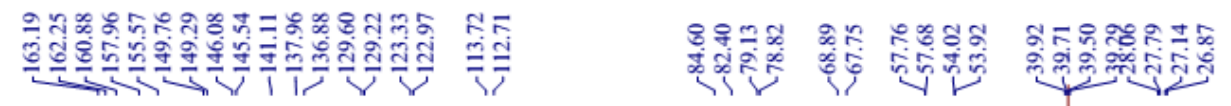

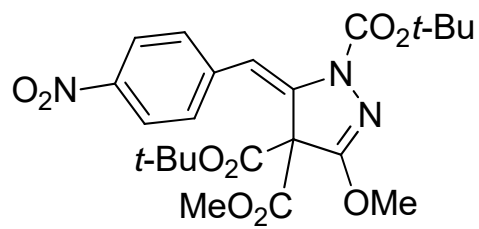

${ }^{13} \mathrm{C}$ NMR $\left(100 \mathrm{MHz}\right.$, DMSO- $\left.d_{6}\right)$

$3 u$

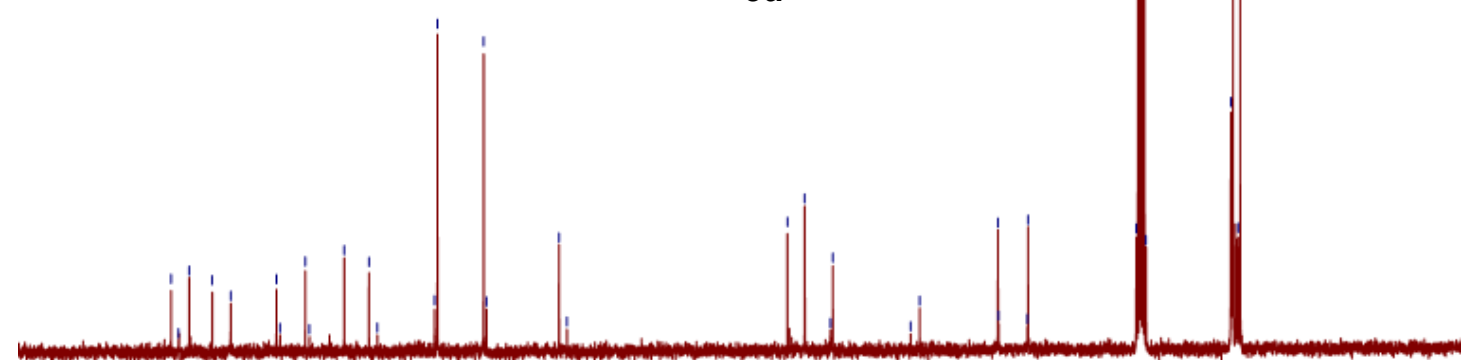

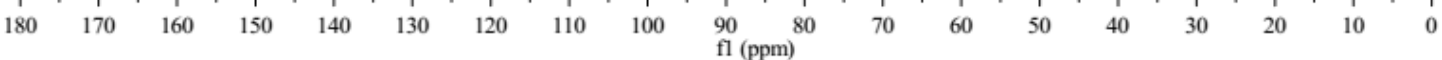



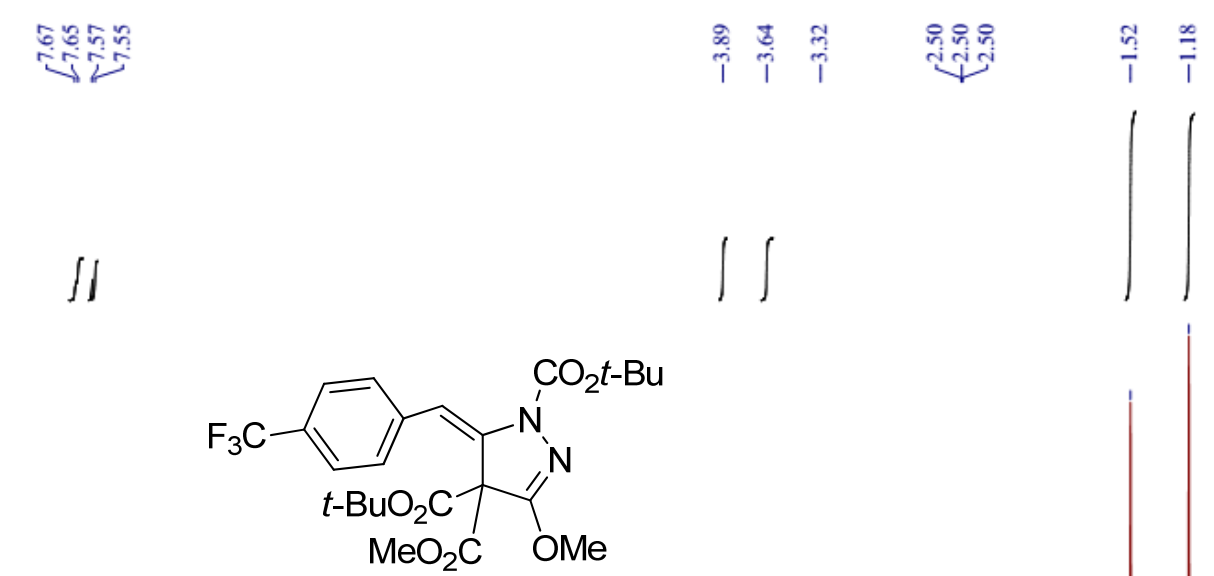

${ }^{1} \mathrm{H}$ NMR $\left(400 \mathrm{MHz}, \mathrm{DMSO}-d_{6}\right)$

3v

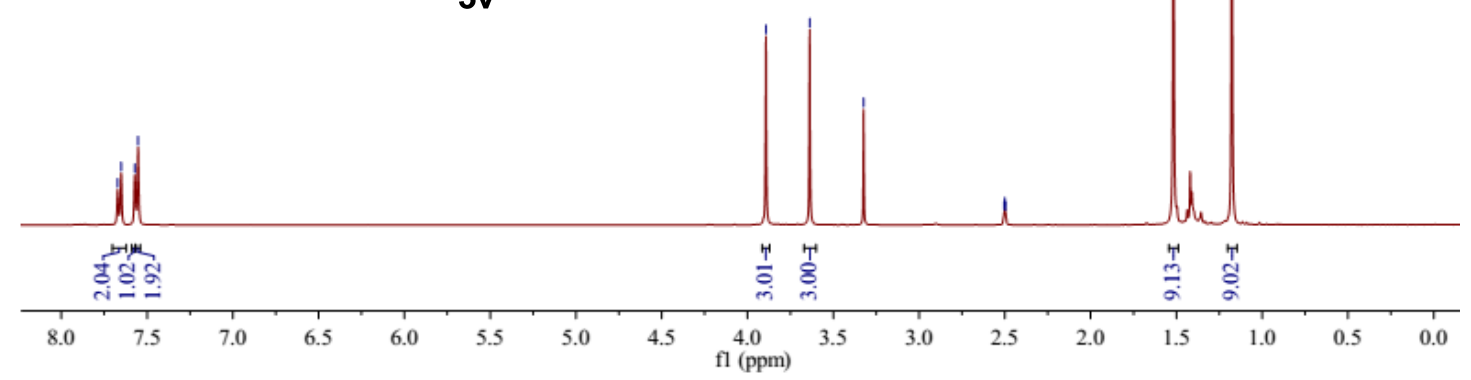

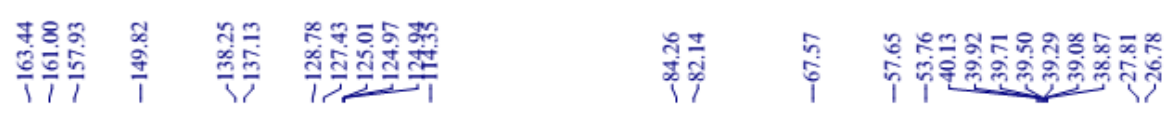

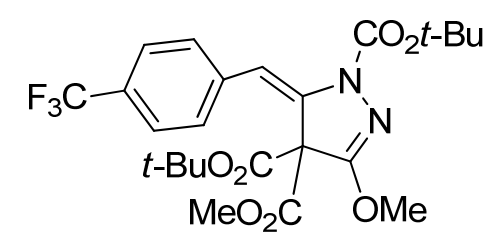

${ }^{13} \mathrm{C}$ NMR (100 MHz, DMSO- $d_{6}$ )

$3 v$

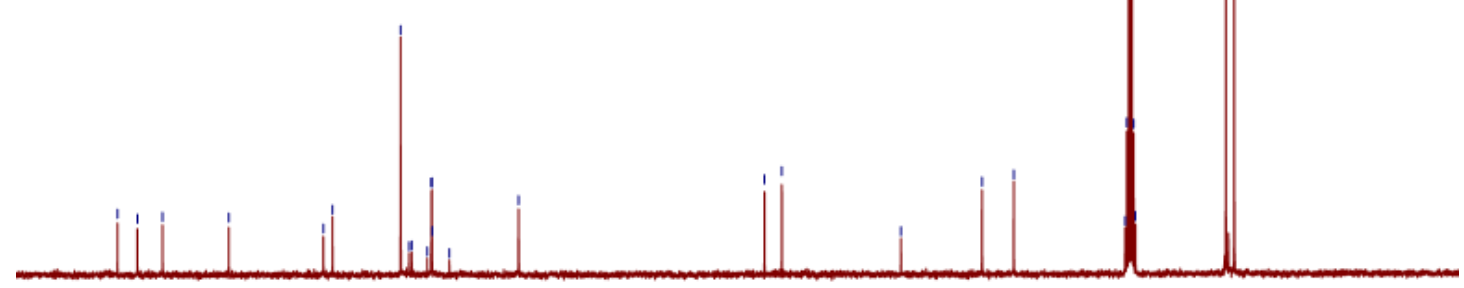

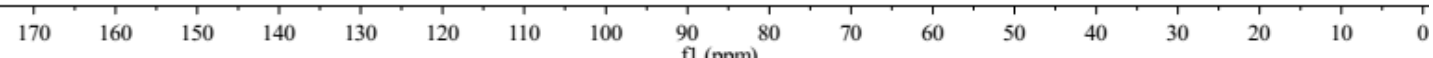




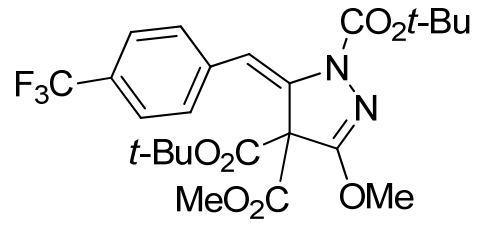

${ }^{19} \mathrm{~F}$ NMR (376 MHz, DMSO-d 6 )

$3 v$

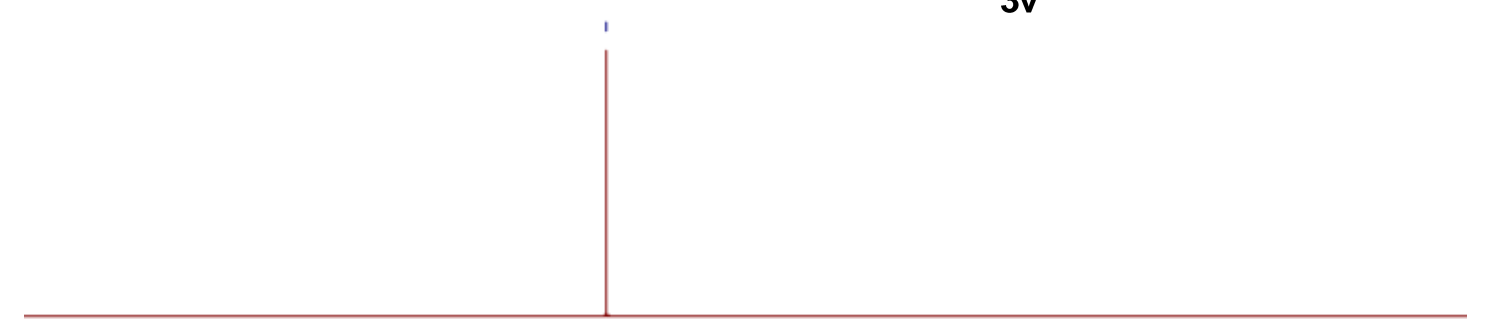

\begin{tabular}{llllllllllllllllllllllllll}
\hline 0 & 40 & 30 & 20 & 10 & 0 & -10 & -20 & -30 & -40 & -50 & -60 & -70 & -80 & -90 & -100 & -120 & -140 & -160 & -180 & -200 & -220
\end{tabular}

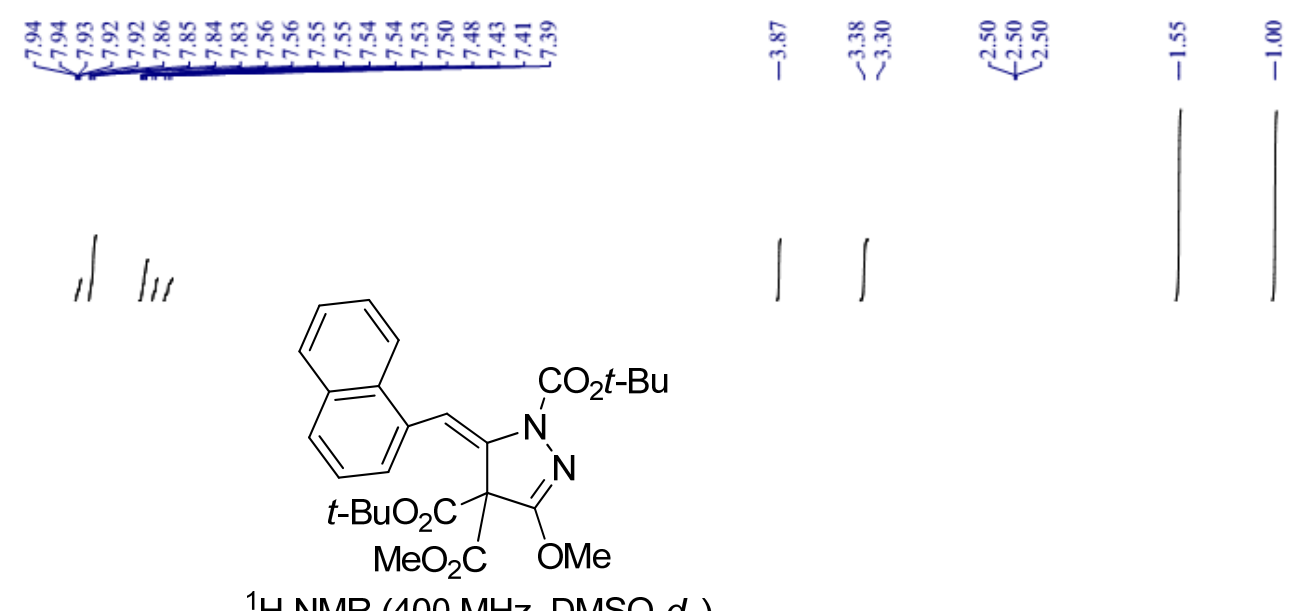

${ }^{1} \mathrm{H}$ NMR (400 MHz, DMSO-d $)$

$3 \mathbf{w}$

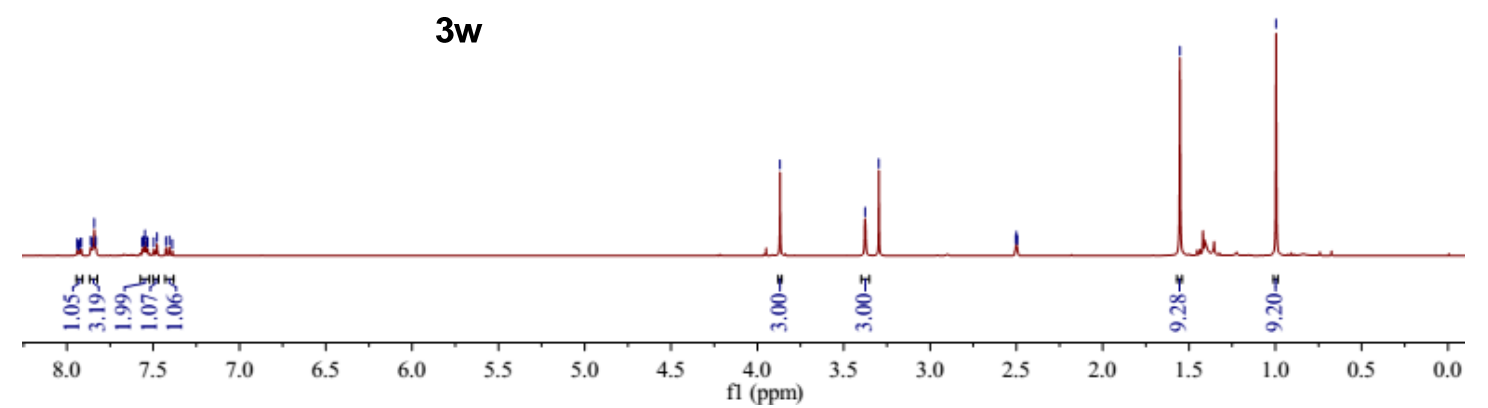




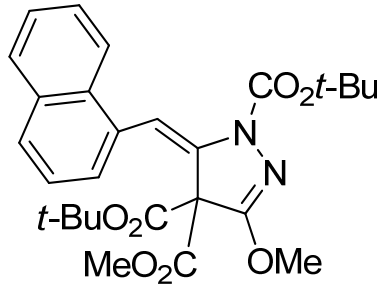

${ }^{13} \mathrm{C}$ NMR (100 MHz, DMSO- $\left.d_{6}\right)$

$3 w$

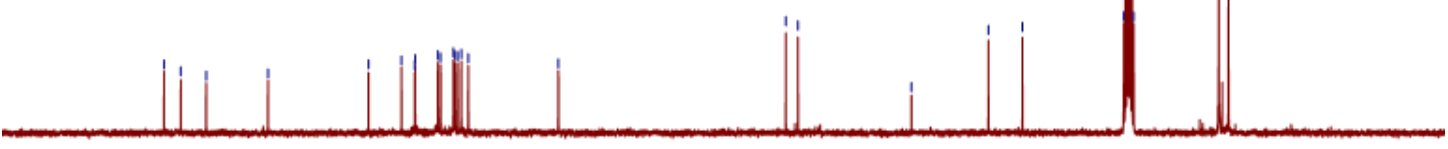

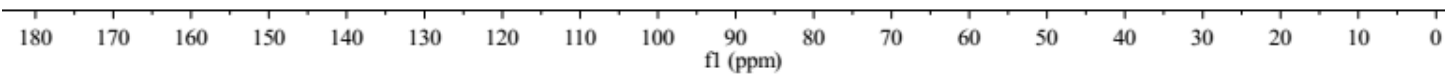

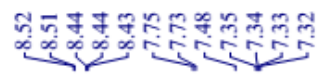

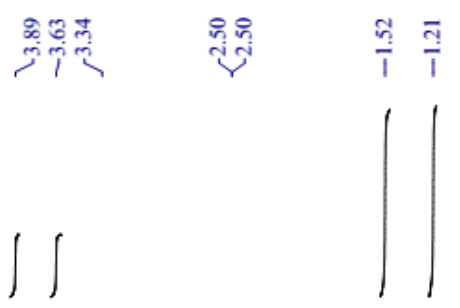

$2 \pi \quad f 2 \pi$<smiles>COC(=O)C1(C(=O)OC)C(OC)=NN(C(=O)OC(C)(C)C)/C1=C\c1ccco1</smiles>

${ }^{1} \mathrm{H}$ NMR $\left(400 \mathrm{MHz}, \mathrm{DMSO}-d_{6}\right)$

$3 \mathbf{x}$

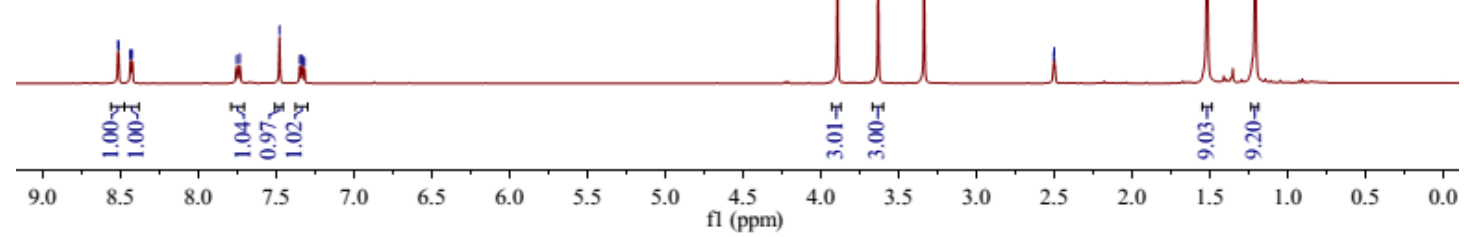




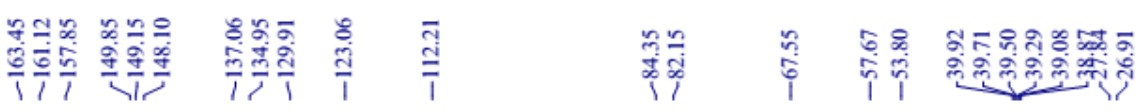

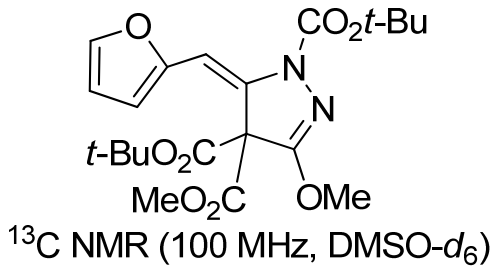

$3 x$
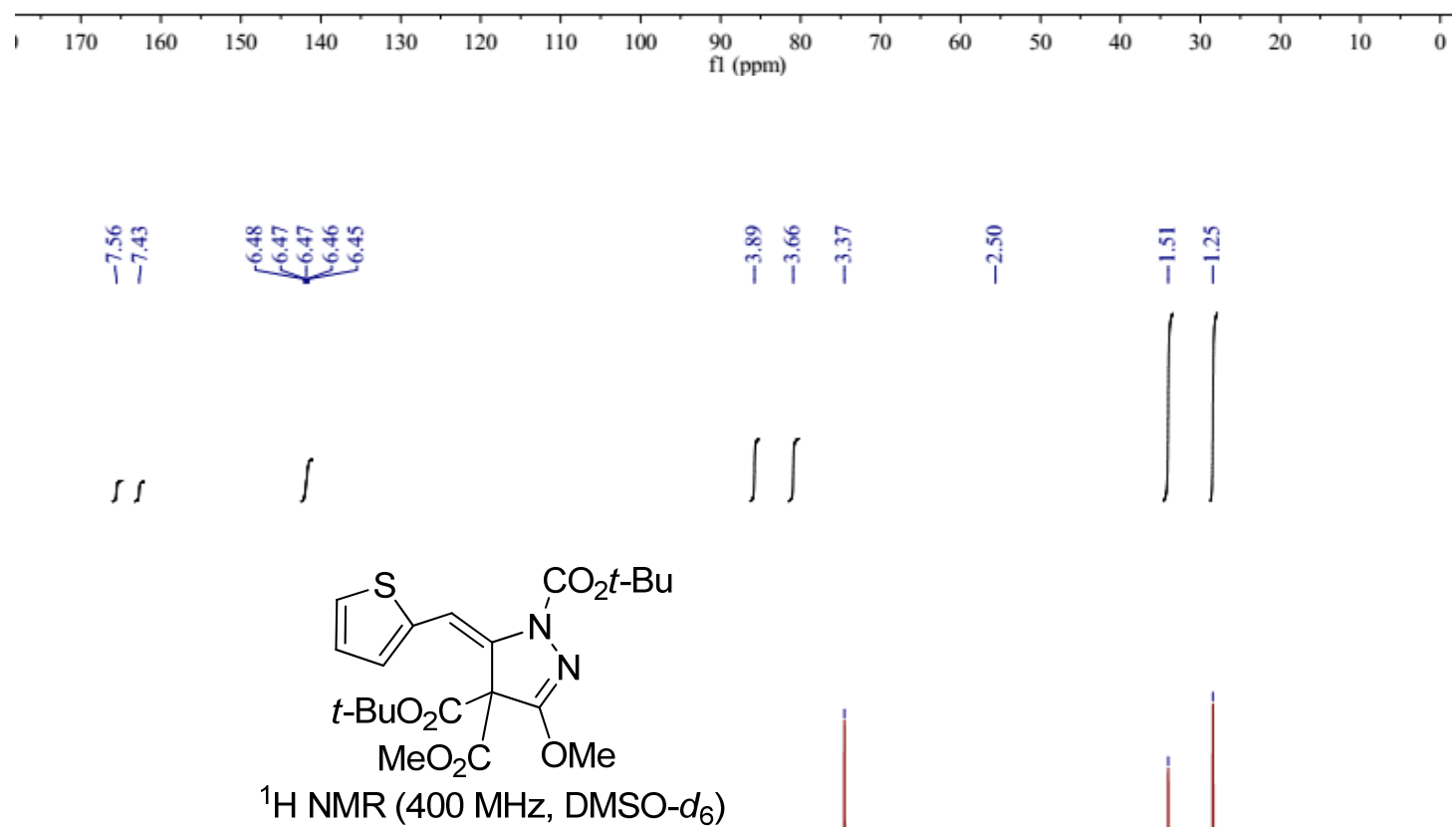

$3 y$

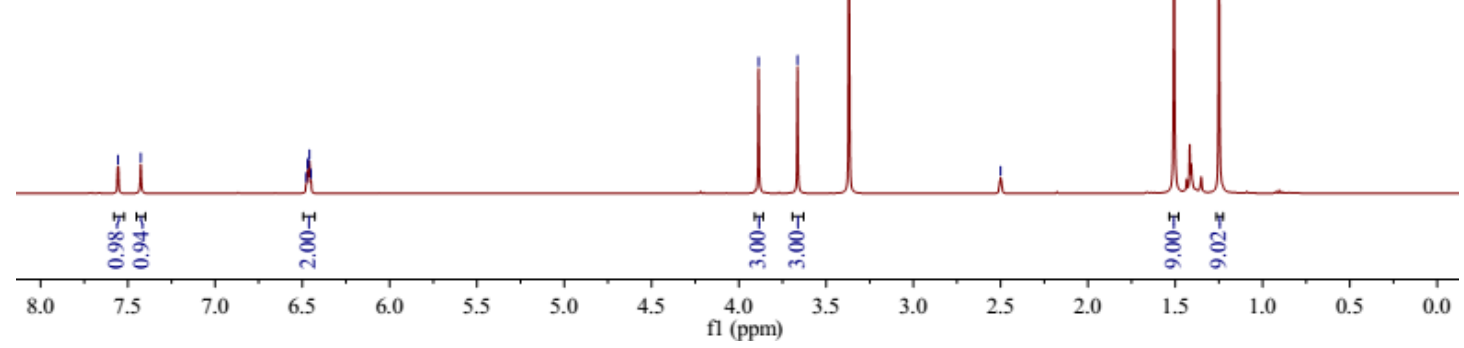




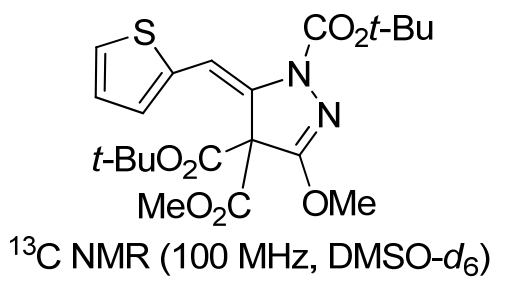

3y
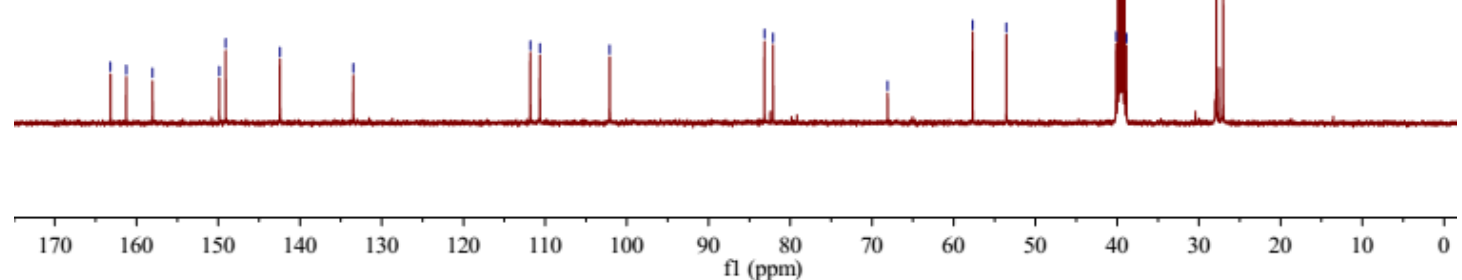

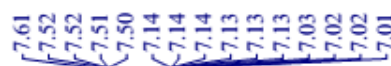

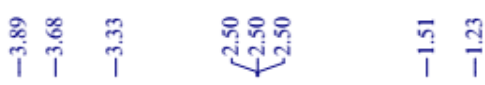

$\int 1 /$

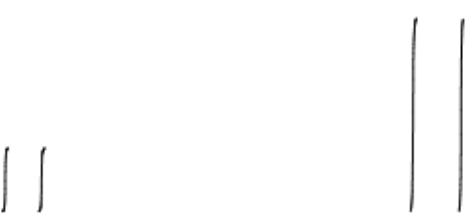<smiles>COC(=O)N1N=C(OC(C)(C)C)C(C)(OC(C)(C)C)C1=Cc1cccnc1</smiles>

${ }^{1} \mathrm{H}$ NMR (400 MHz, DMSO- $d_{6}$ )

$3 z$

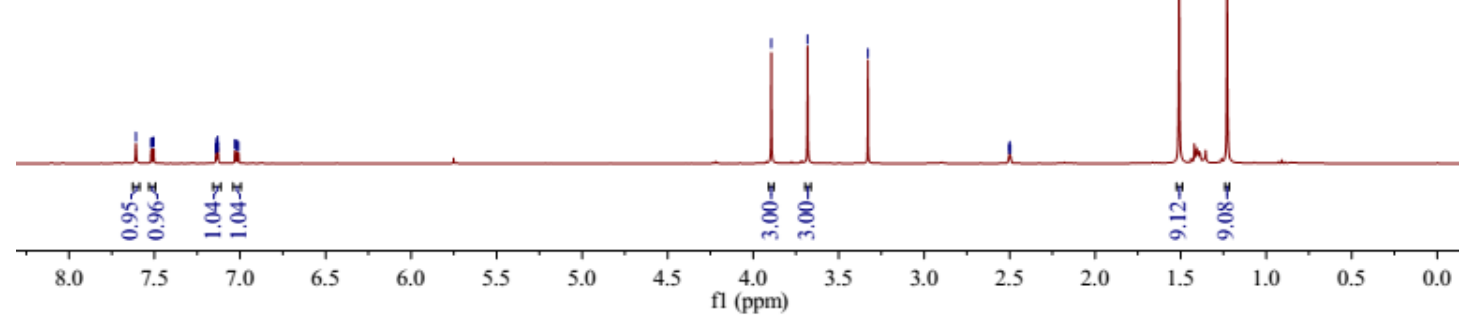




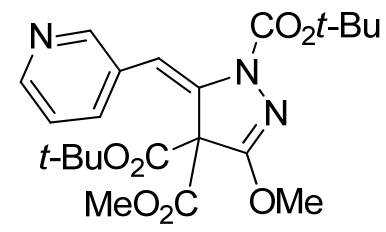

${ }^{13} \mathrm{C}$ NMR (100 MHz, DMSO- $d_{6}$ )

$3 z$

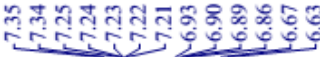

$\iint s$<smiles>COC(=O)C1(C(=O)OC)C(=CC=Cc2ccccc2)N(C(=O)OC(C)(C)C)N=C1C(C)(C)C</smiles>

${ }^{1} \mathrm{H}$ NMR (400 MHz, DMSO- $d_{6}$ )

3A

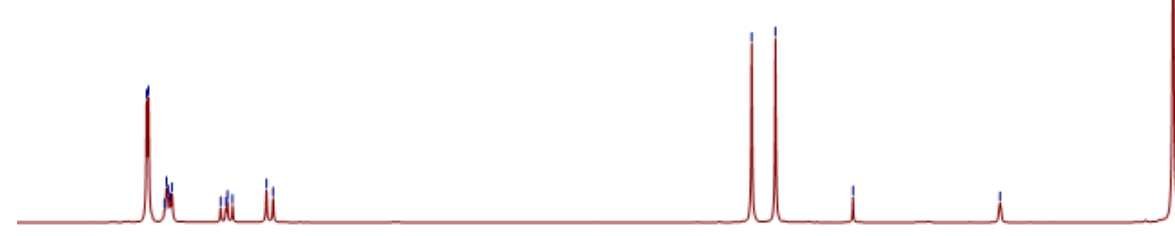

\begin{tabular}{|c|c|c|c|c|c|c|c|c|c|c|c|c|c|c|c|c|}
\hline & 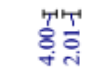 & $\begin{array}{l}\text { TT' } \\
8 \\
\end{array}$ & $\underset{8}{\top}$ & & & & & 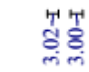 & & & & & $\frac{T}{T} \frac{T}{2}$ & & & \\
\hline 8.0 & 7.5 & 7.0 & 6.5 & 6.0 & 5.5 & 5.0 & 4.5 & $\begin{array}{c}4.0 \\
\mathrm{fl}(\mathrm{ppm})\end{array}$ & 3.5 & 3.0 & 2.5 & 2.0 & 1.5 & 1.0 & 0.5 & 0.0 \\
\hline
\end{tabular}




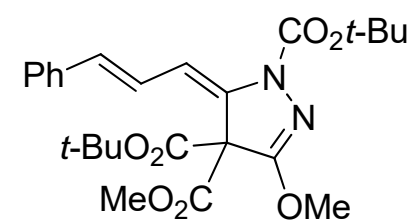

${ }^{13} \mathrm{C}$ NMR (100 MHz, DMSO- $d_{6}$ )

$3 A$
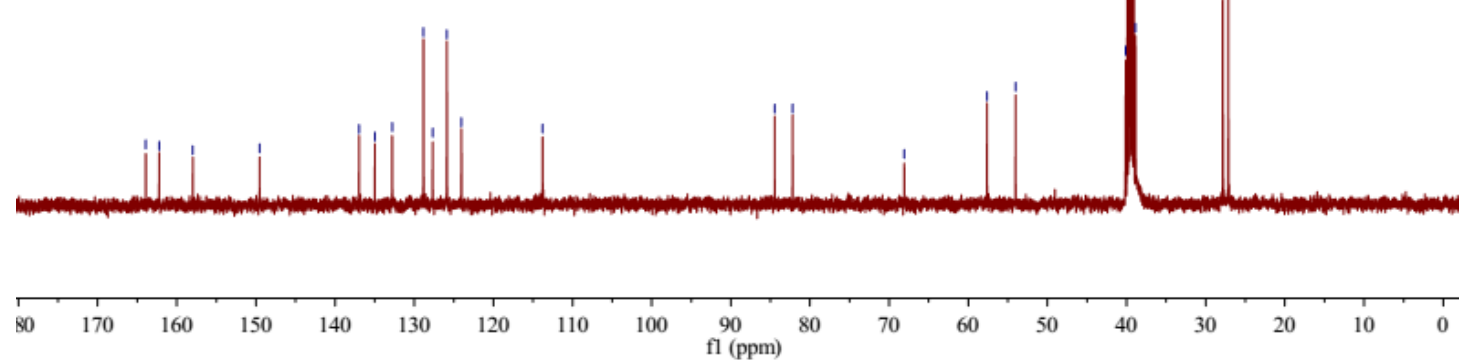

$\stackrel{8}{1}$
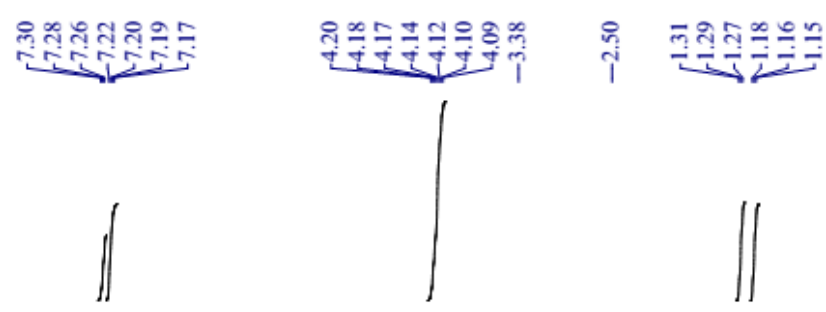<smiles>CCOc1n[nH]c(Cc2ccccc2)c1OCC</smiles>

${ }^{1} \mathrm{H}$ NMR (400 MHz, DMSO- $d_{6}$ )

4a

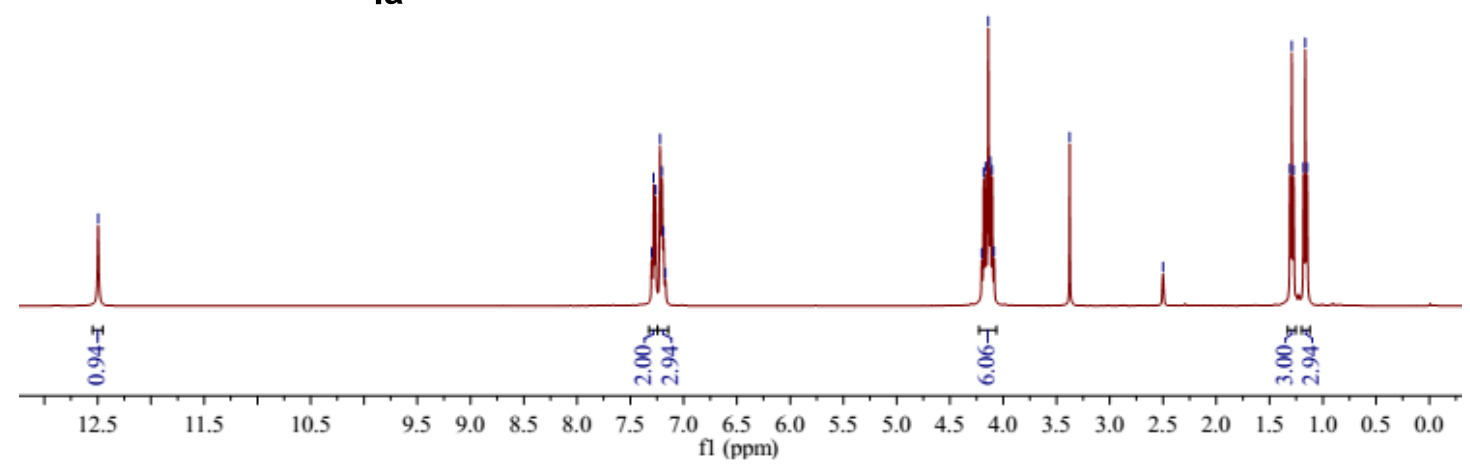




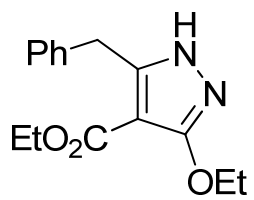

${ }^{13} \mathrm{C}$ NMR (100 MHz, DMSO- $\left.d_{6}\right)$

$4 a$
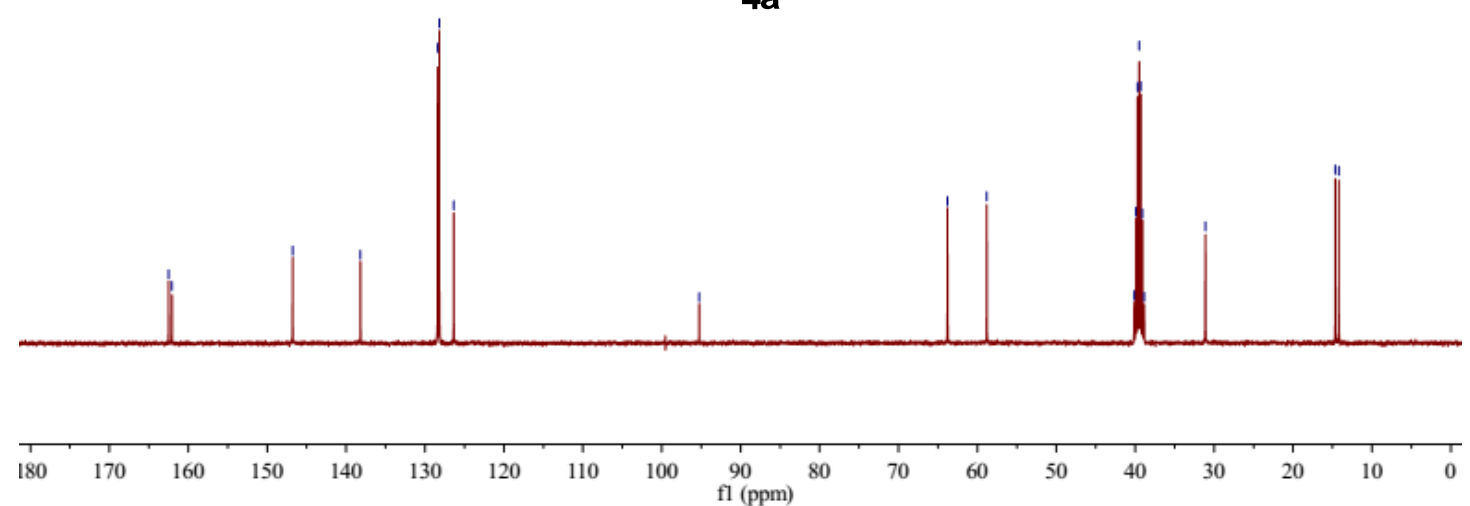

$\stackrel{\infty}{\frac{\infty}{3}}$

ㅇำ

गंक्षि क्ष

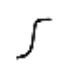
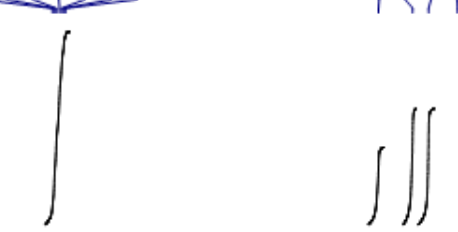<smiles>COC(=O)c1c(OC)n[nH]c1Cc1ccccc1</smiles>

${ }^{1} \mathrm{H}$ NMR (400 MHz, DMSO- $d_{6}$ )

4b

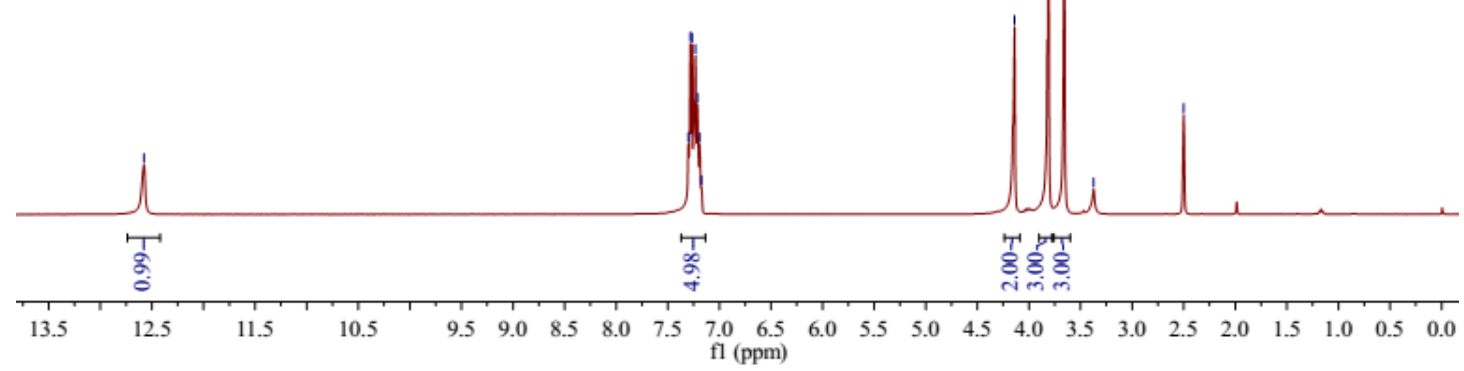



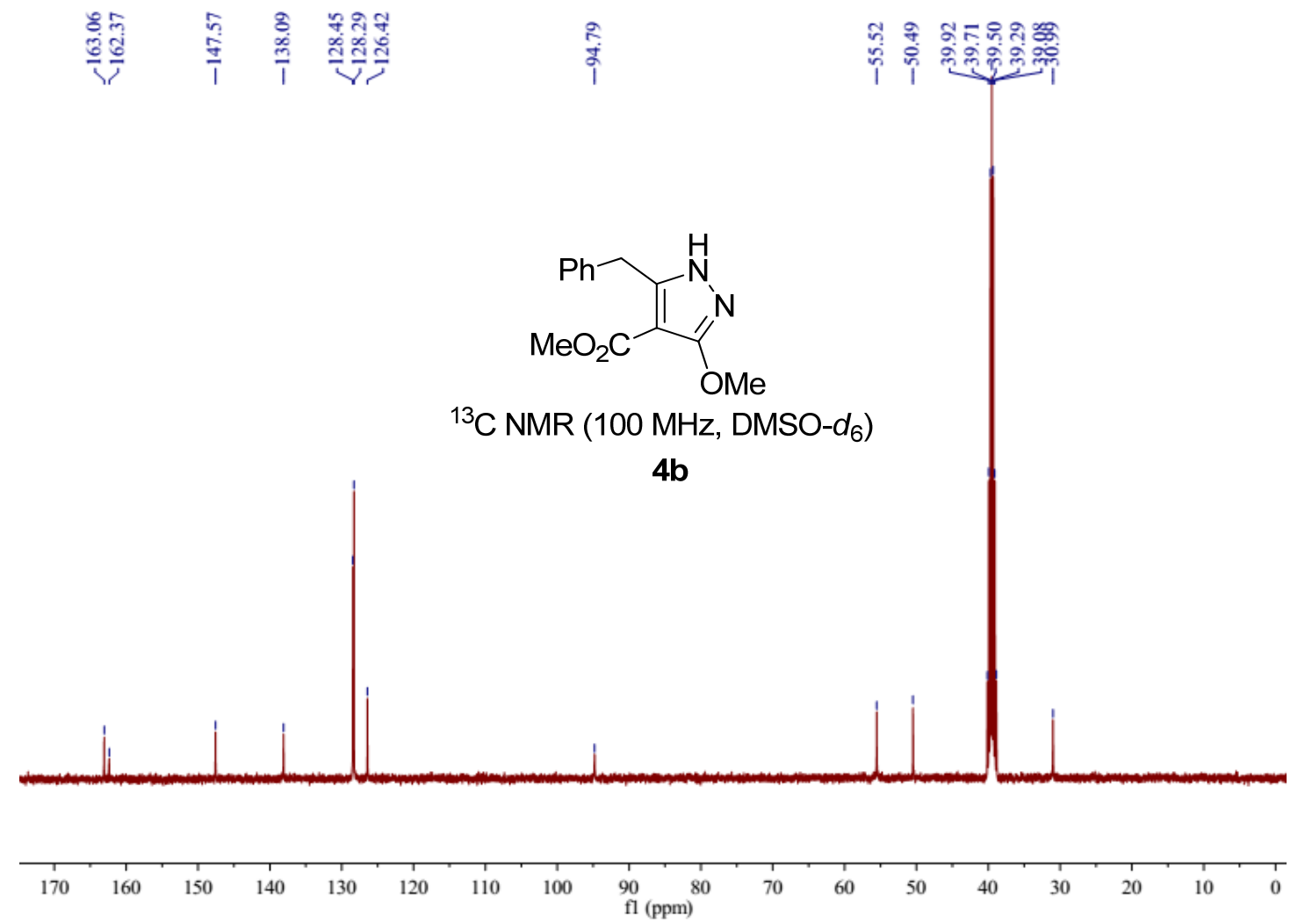

i

j
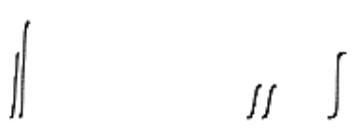<smiles>CCCOc1n[nH]c(Cc2ccccc2)c1C(=O)O[In]</smiles>

${ }^{1} \mathrm{H}$ NMR (400 MHz, DMSO- $d_{6}$ )

4c

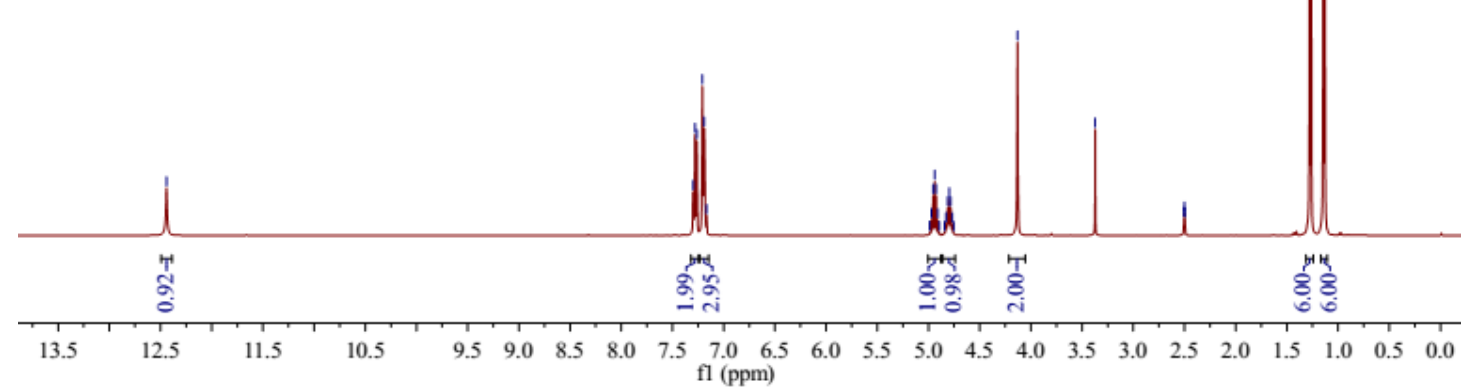




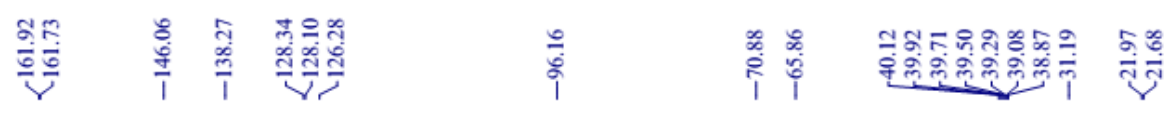
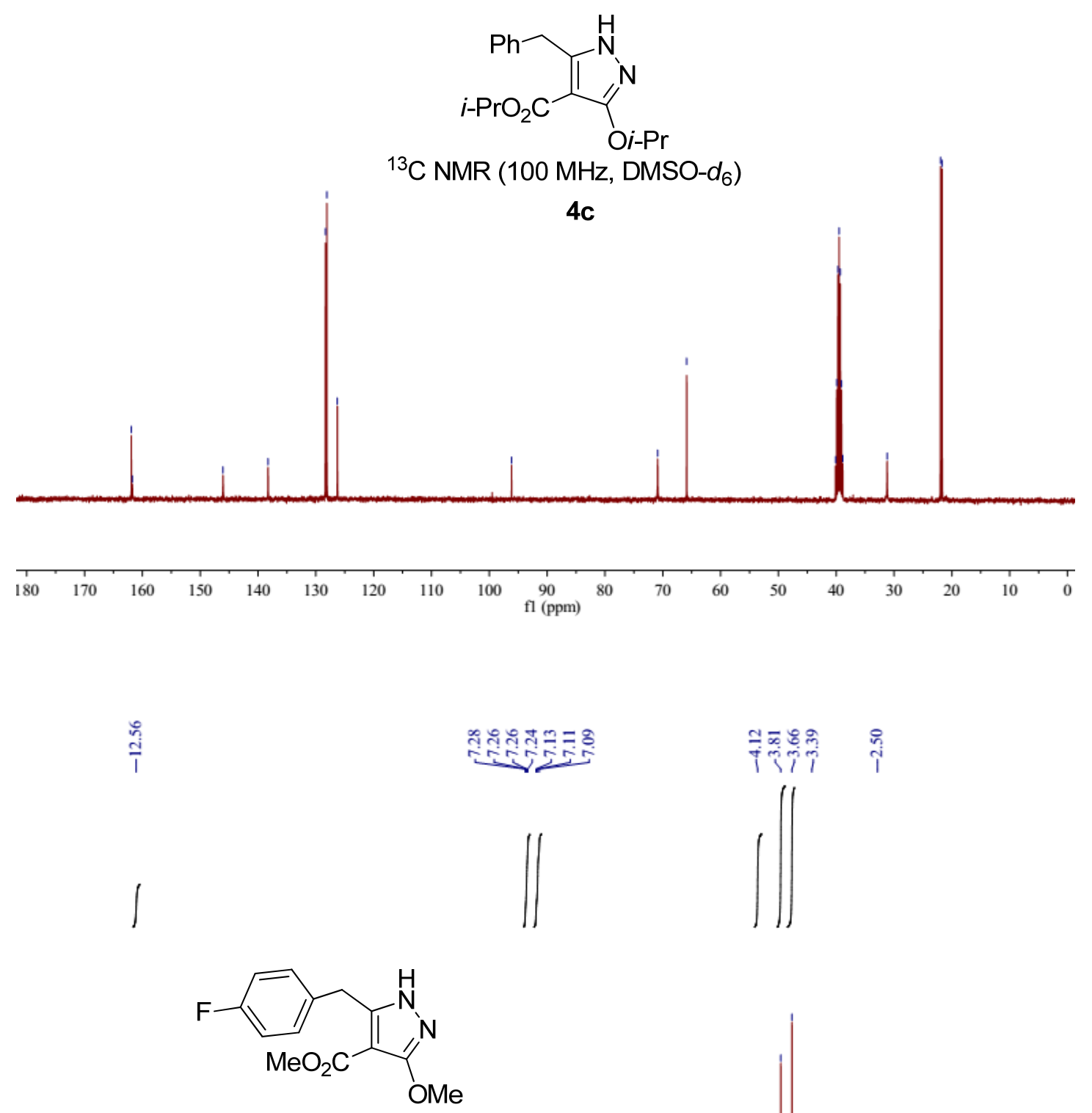

${ }^{1} \mathrm{H}$ NMR $\left(400 \mathrm{MHz}\right.$, DMSO- $\left.d_{6}\right)$

$4 d$

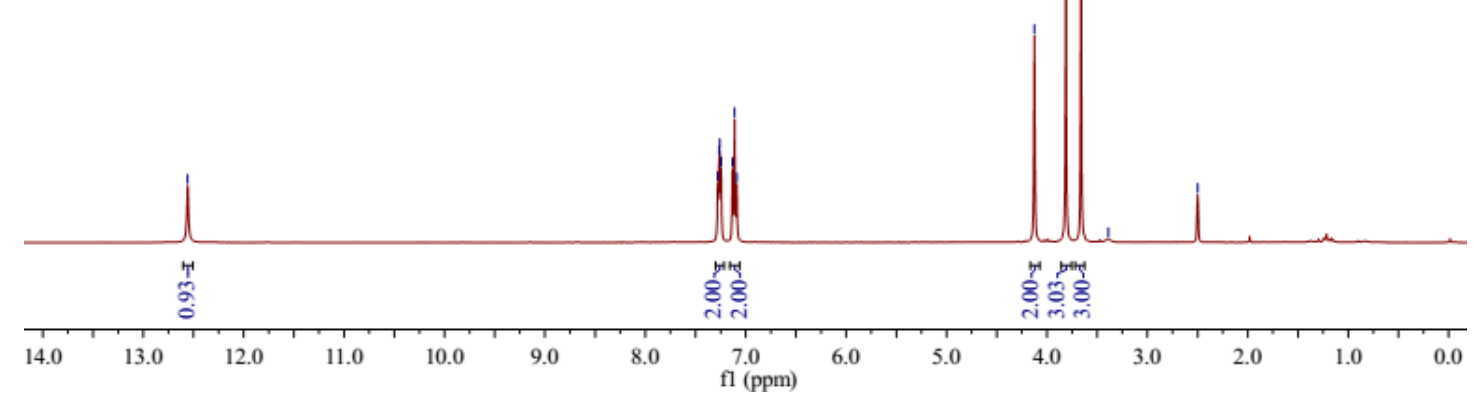




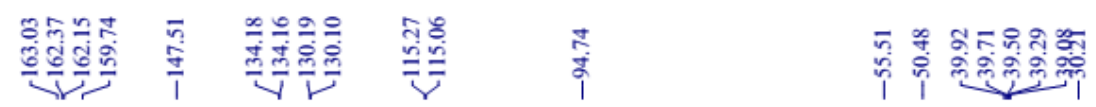

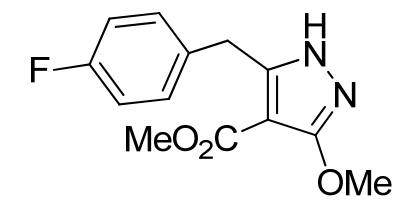

${ }^{13} \mathrm{C}$ NMR (100 MHz, DMSO- $d_{6}$ )

4d
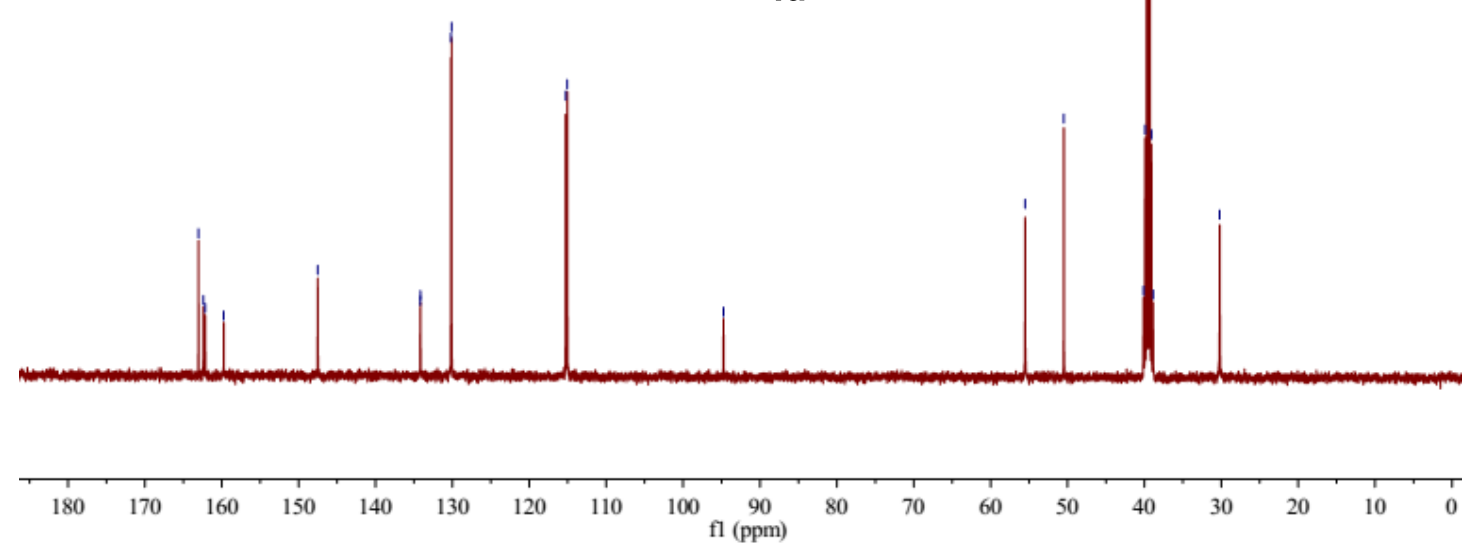

$\stackrel{\bar{\oplus}}{\bar{i}}$<smiles>COC(=O)c1c(OC)n[nH]c1Cc1ccc(F)cc1</smiles>

${ }^{19} \mathrm{~F}$ NMR (376 MHz, DMSO- $d_{6}$ )

4d

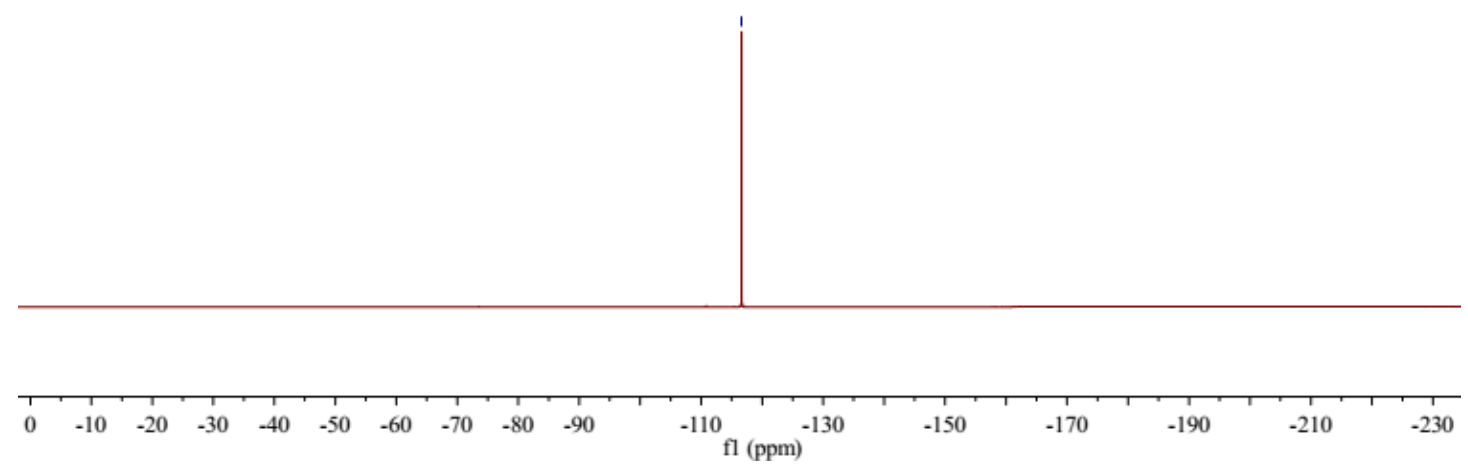




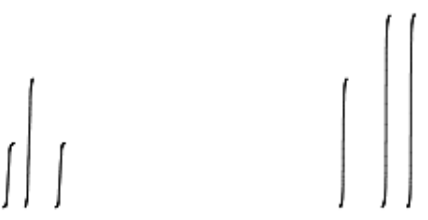<smiles>COC(=O)c1c(OC)n[nH]c1Cc1ccccc1Cl</smiles>

${ }^{1} \mathrm{H}$ NMR (400 MHz, DMSO- $\left.d_{6}\right)$

4 e

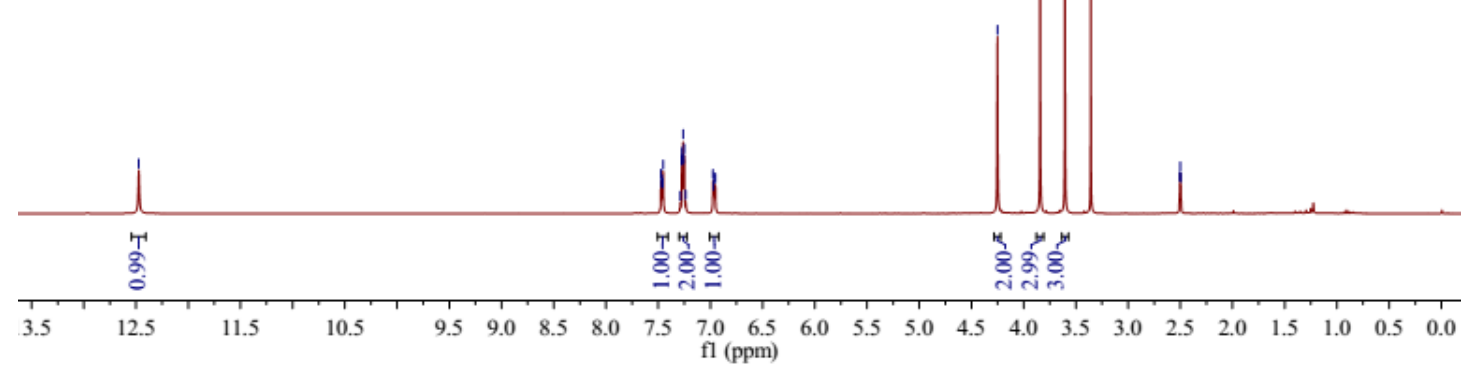

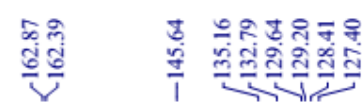

i

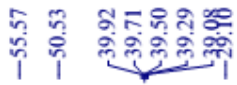

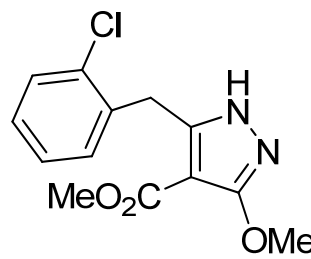

${ }^{13} \mathrm{C}$ NMR (100 MHz, DMSO-d $\left.{ }_{6}\right)$

$4 e$

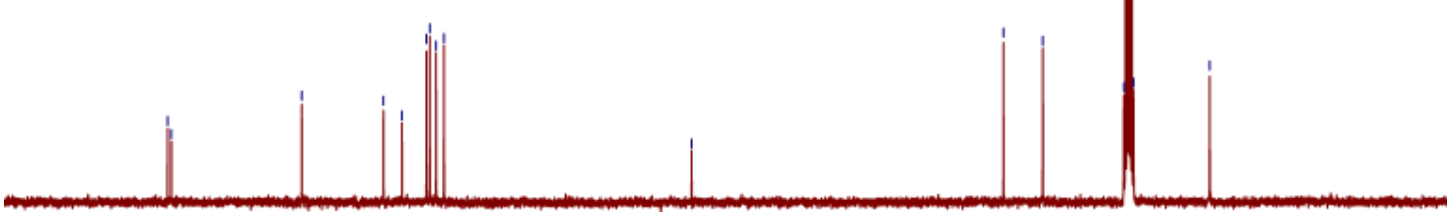

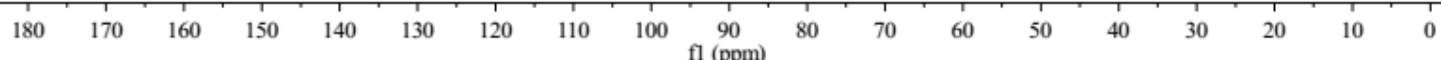



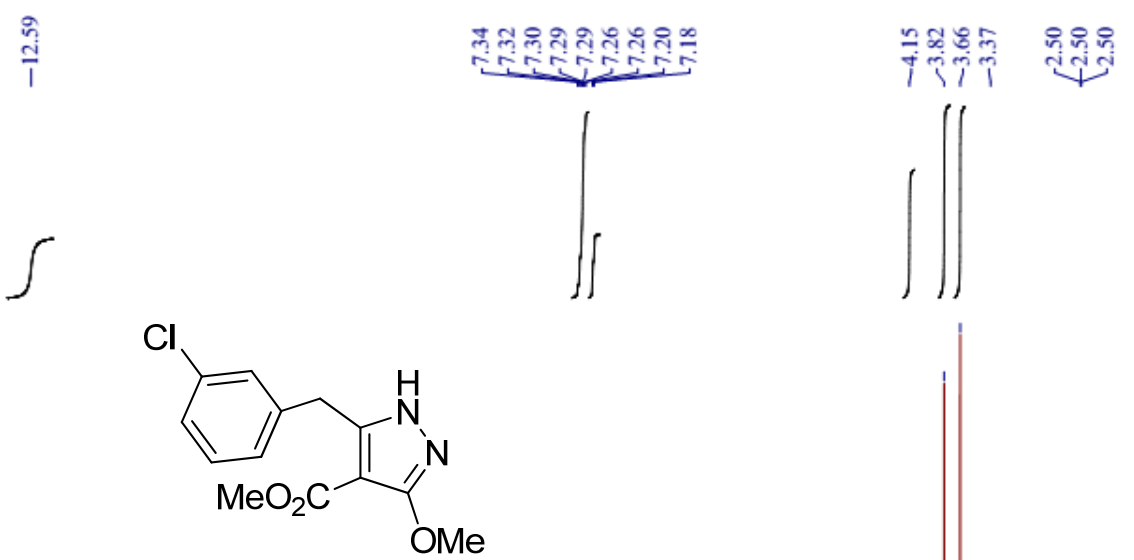

${ }^{1} \mathrm{H}$ NMR $\left(400 \mathrm{MHz}, \mathrm{DMSO}-d_{6}\right)$

$4 f$

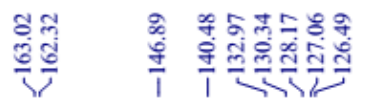

$\stackrel{\infty}{i}$

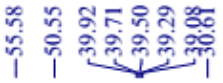

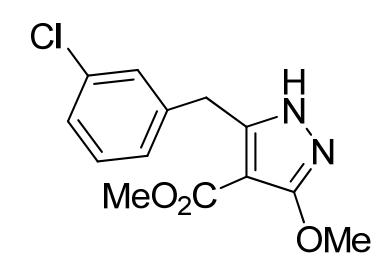

${ }^{13} \mathrm{C}$ NMR (100 MHz, DMSO- $d_{6}$ )

$4 f$

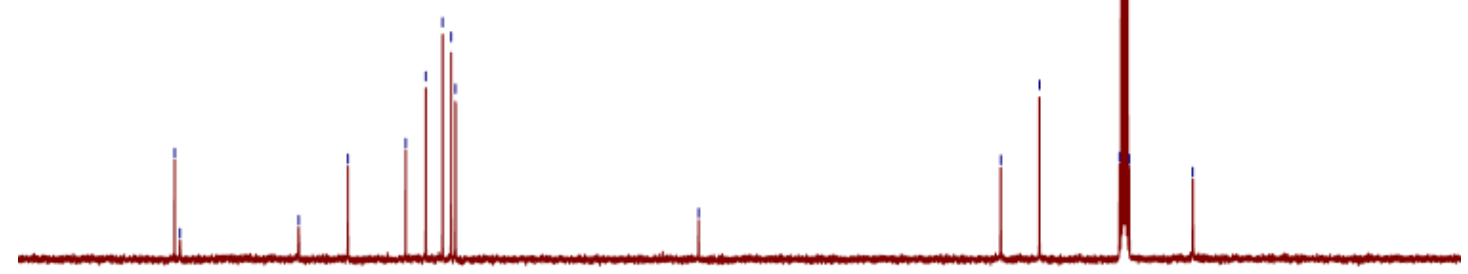

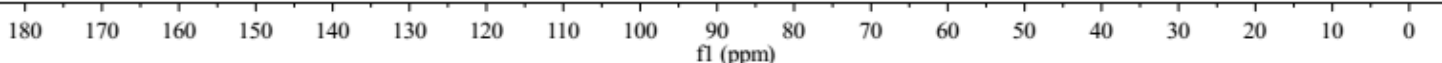


กิ่

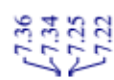

वक्ष

$\int$
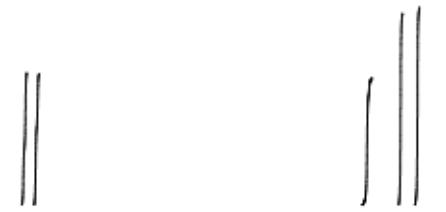<smiles>COC(=O)c1c(OC)n[nH]c1Cc1ccc(Cl)cc1</smiles>

${ }^{1} \mathrm{H}$ NMR (400 MHz, DMSO-d ${ }_{6}$ )

$\mathbf{4 g}$

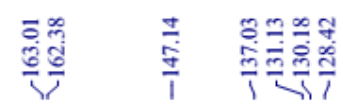

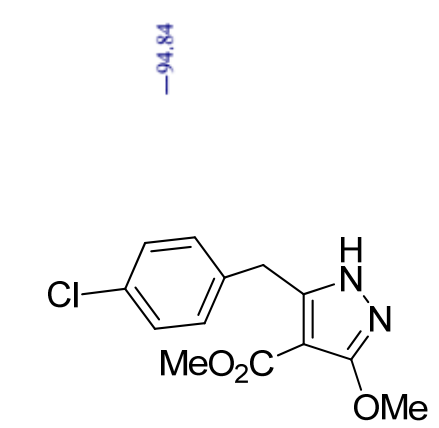

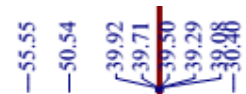

${ }^{13} \mathrm{C}$ NMR (100 MHz, DMSO- $\left.d_{6}\right)$

$4 \mathrm{~g}$

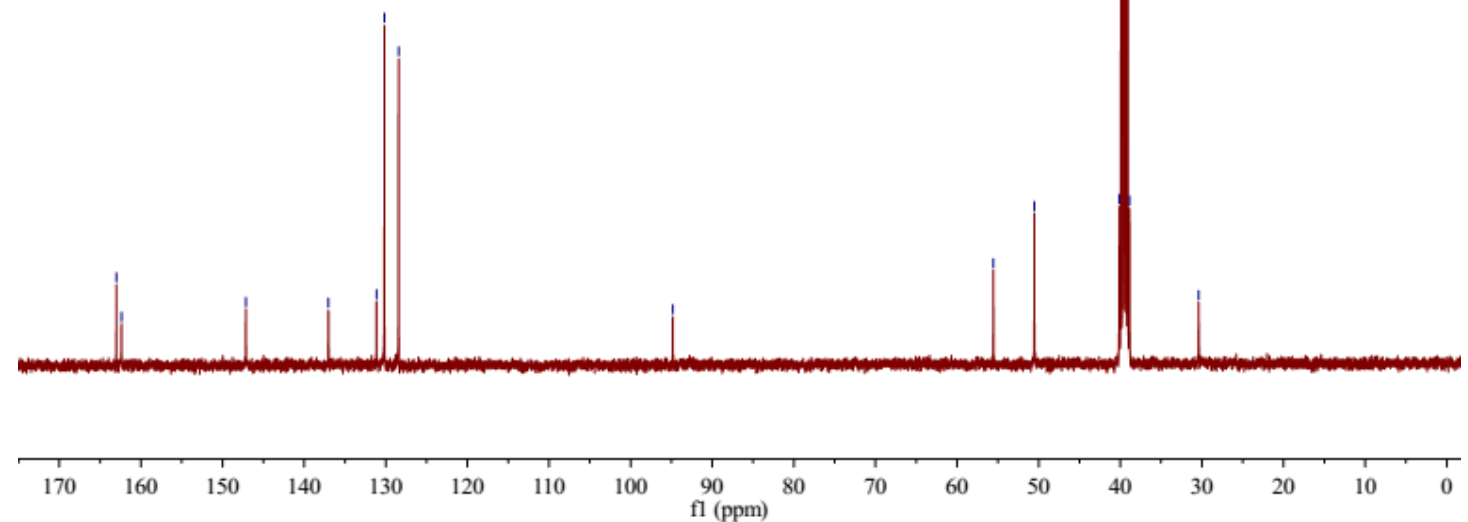



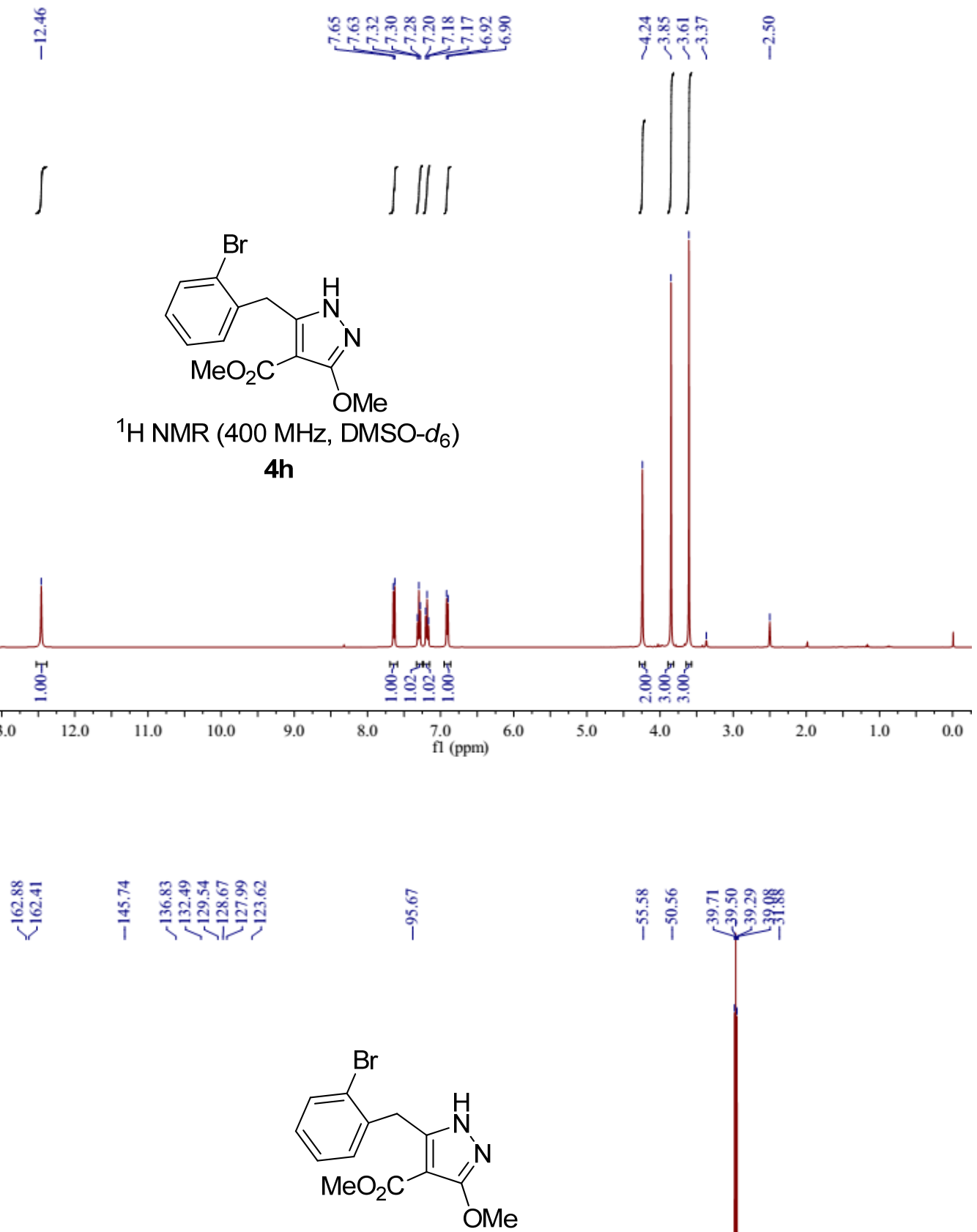

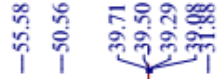

${ }^{13} \mathrm{C}$ NMR $\left(100 \mathrm{MHz}\right.$, DMSO- $\left.d_{6}\right)$

$4 \mathrm{~h}$

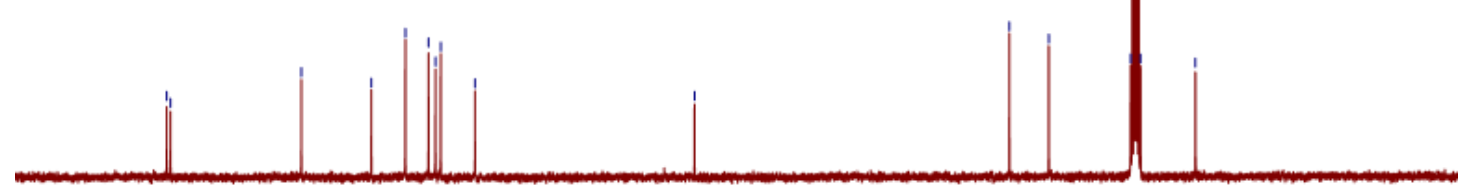

$180 \quad 17$

$\begin{array}{llll}160 & 150 & 140 & 130\end{array}$

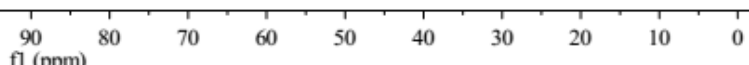



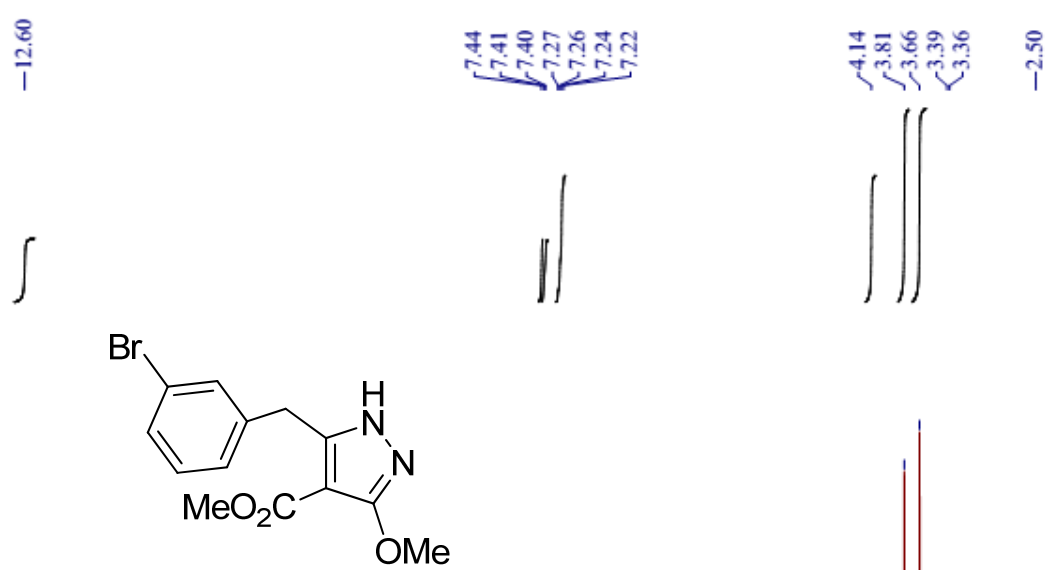

${ }^{1} \mathrm{H}$ NMR (400 MHz, DMSO-d $\left.d_{6}\right)$

4i
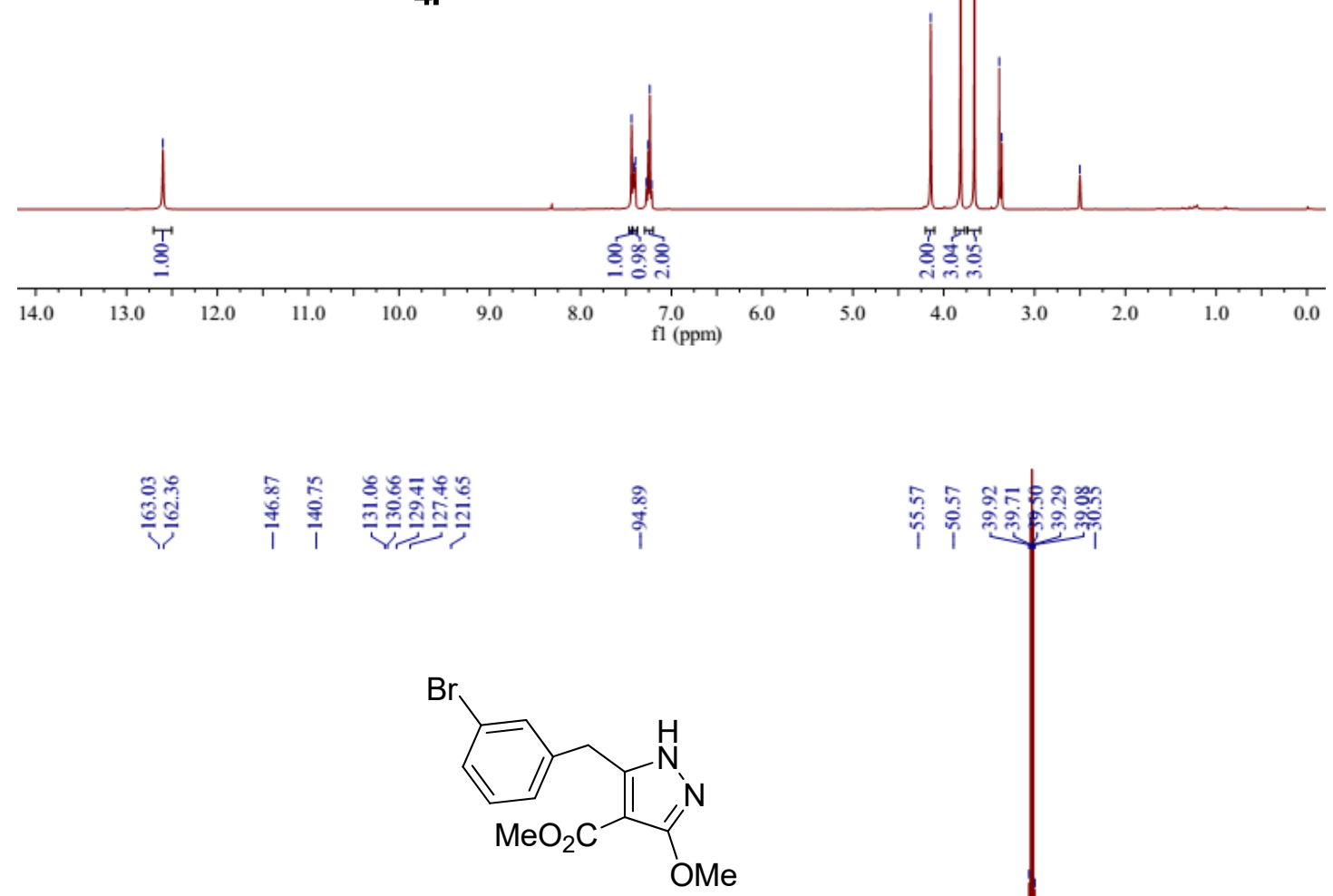

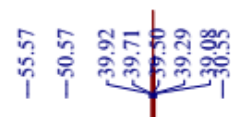

${ }^{13} \mathrm{C}$ NMR $\left(100 \mathrm{MHz}\right.$, DMSO- $\left.d_{6}\right)$

$4 \mathbf{i}$

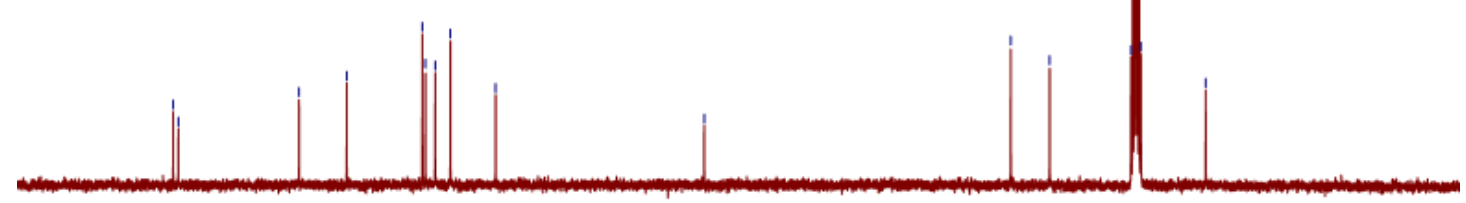

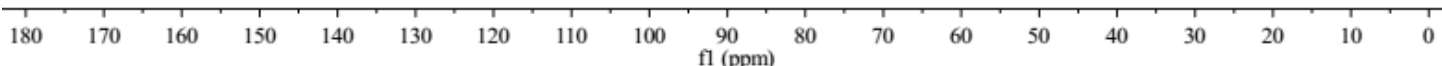




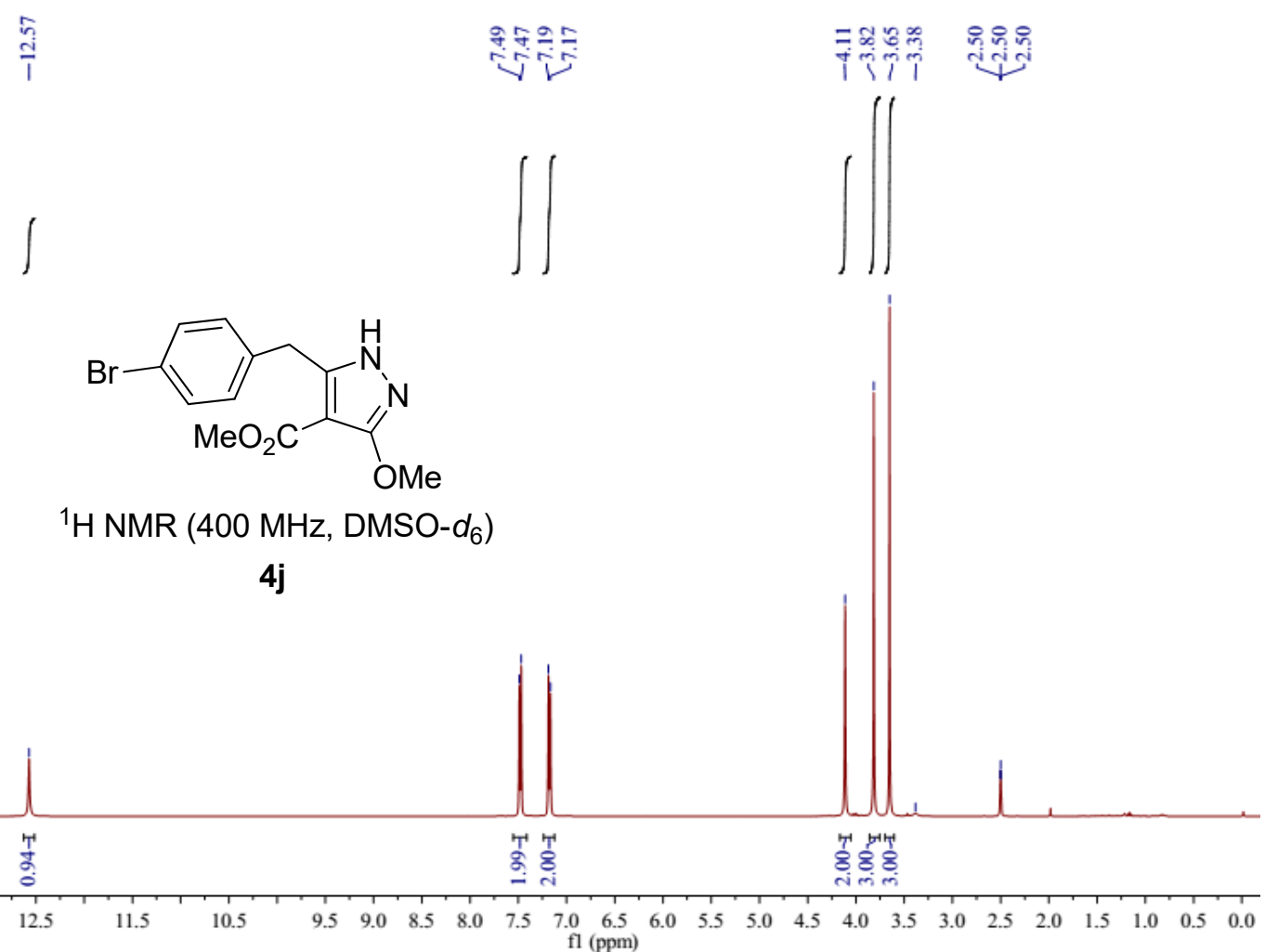

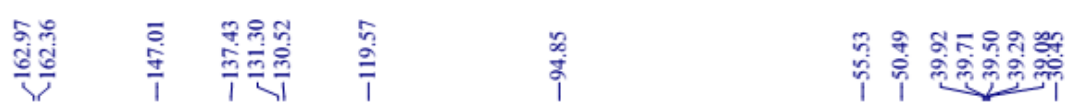
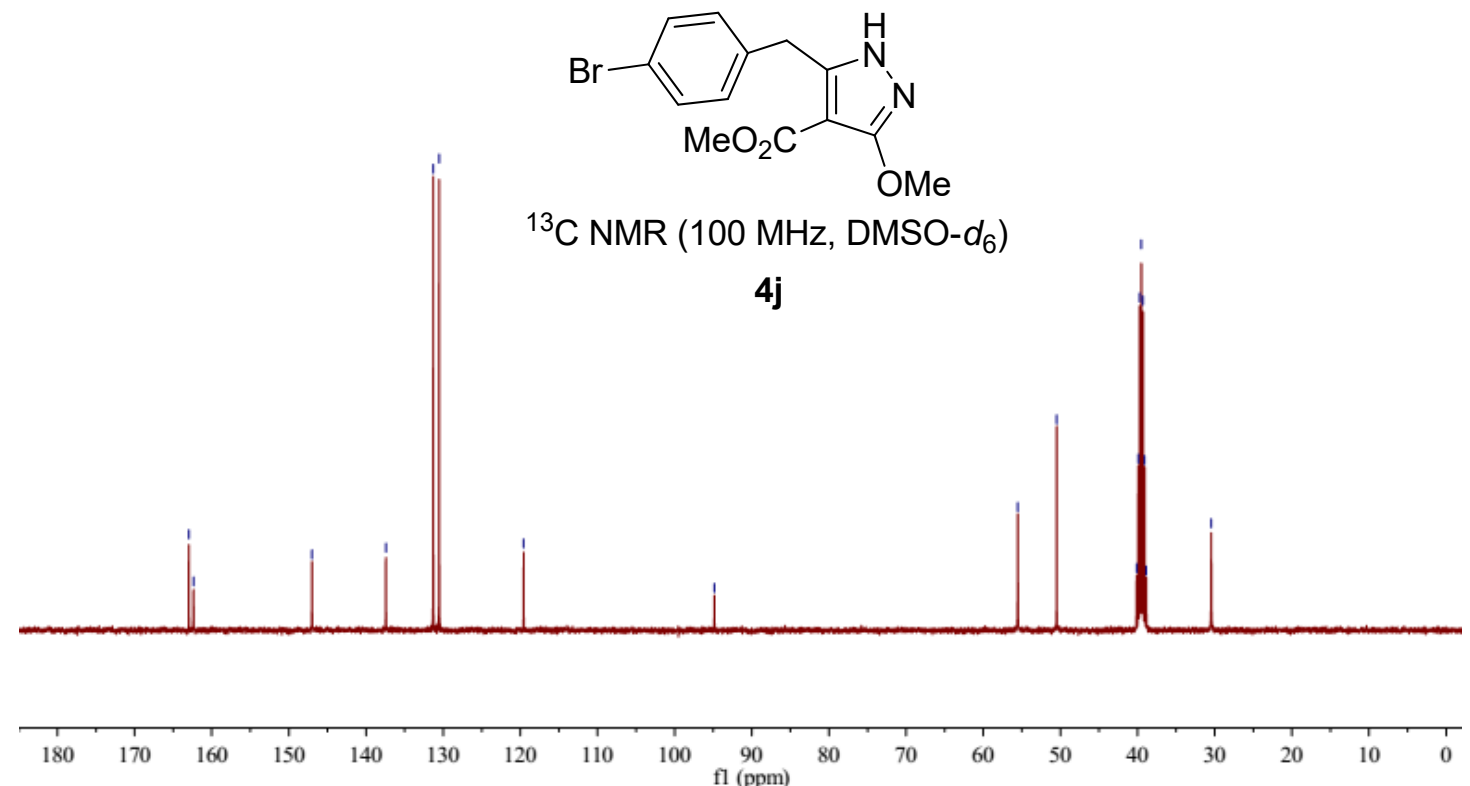

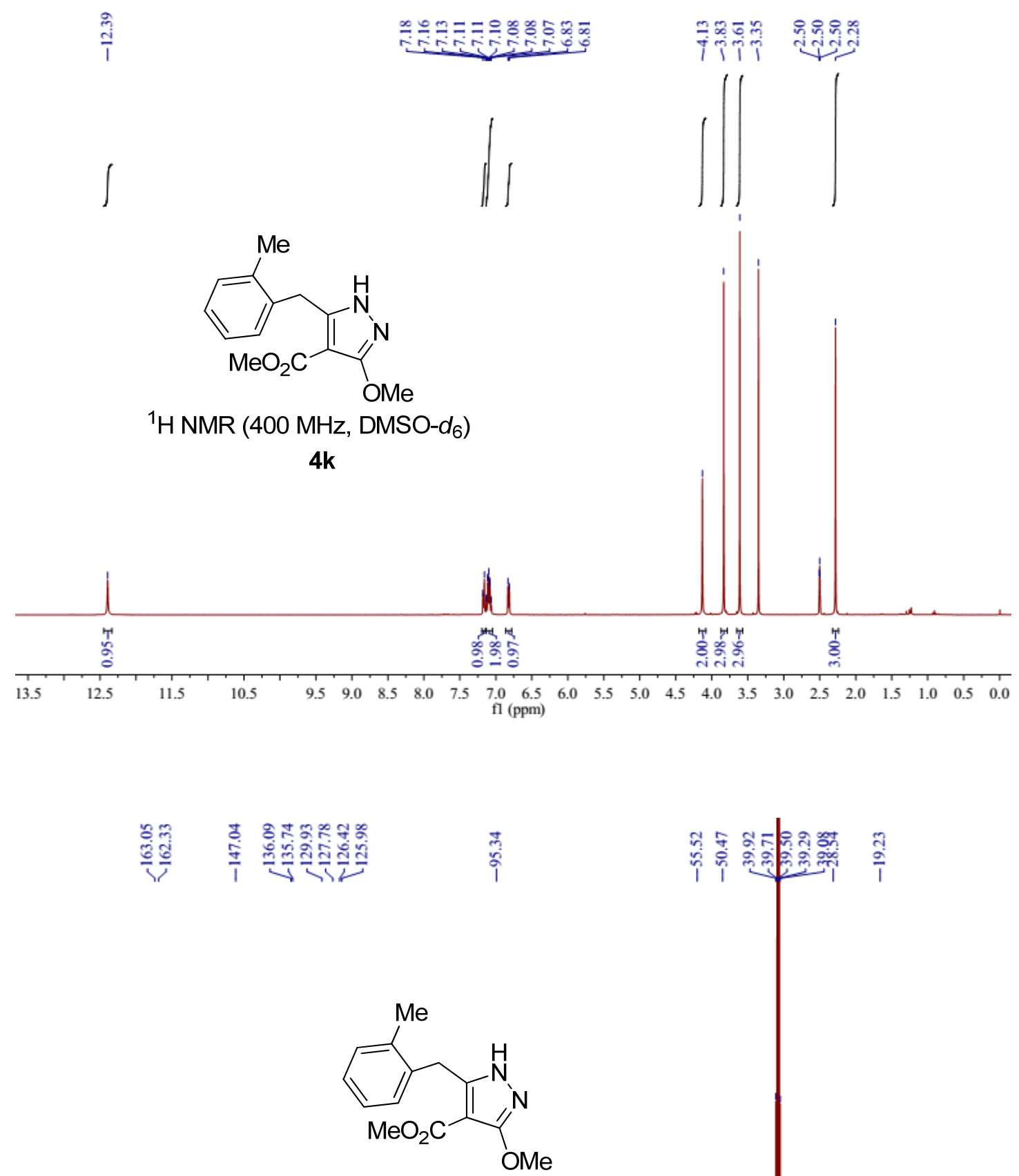

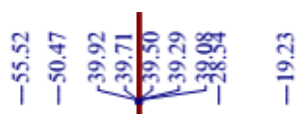

${ }^{13} \mathrm{C}$ NMR $\left(100 \mathrm{MHz}\right.$, DMSO- $\left.d_{6}\right)$

4k

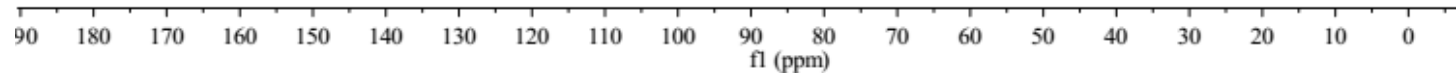



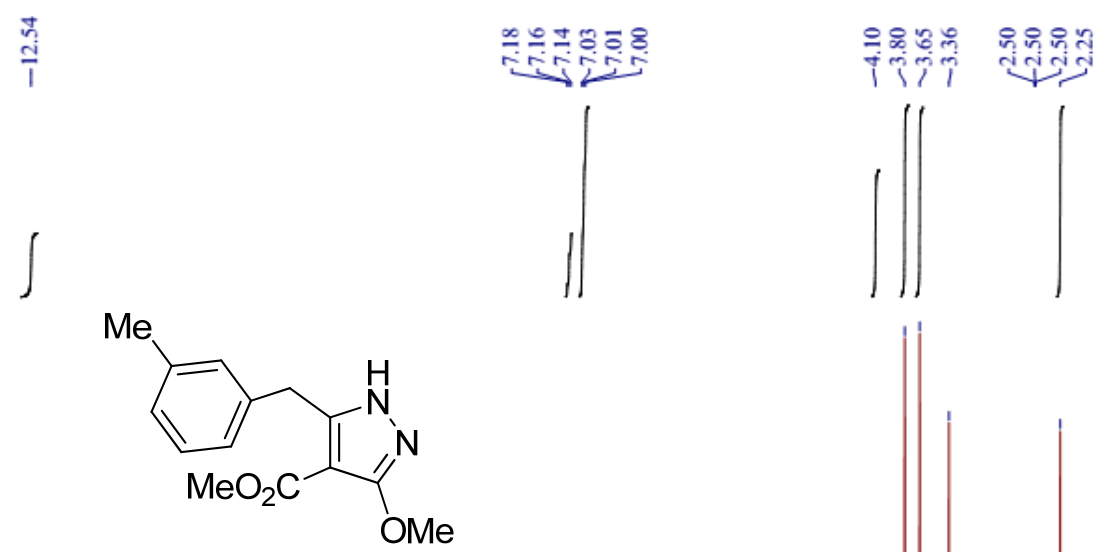

${ }^{1} \mathrm{H}$ NMR (400 MHz, DMSO- $\left.d_{6}\right)$

41
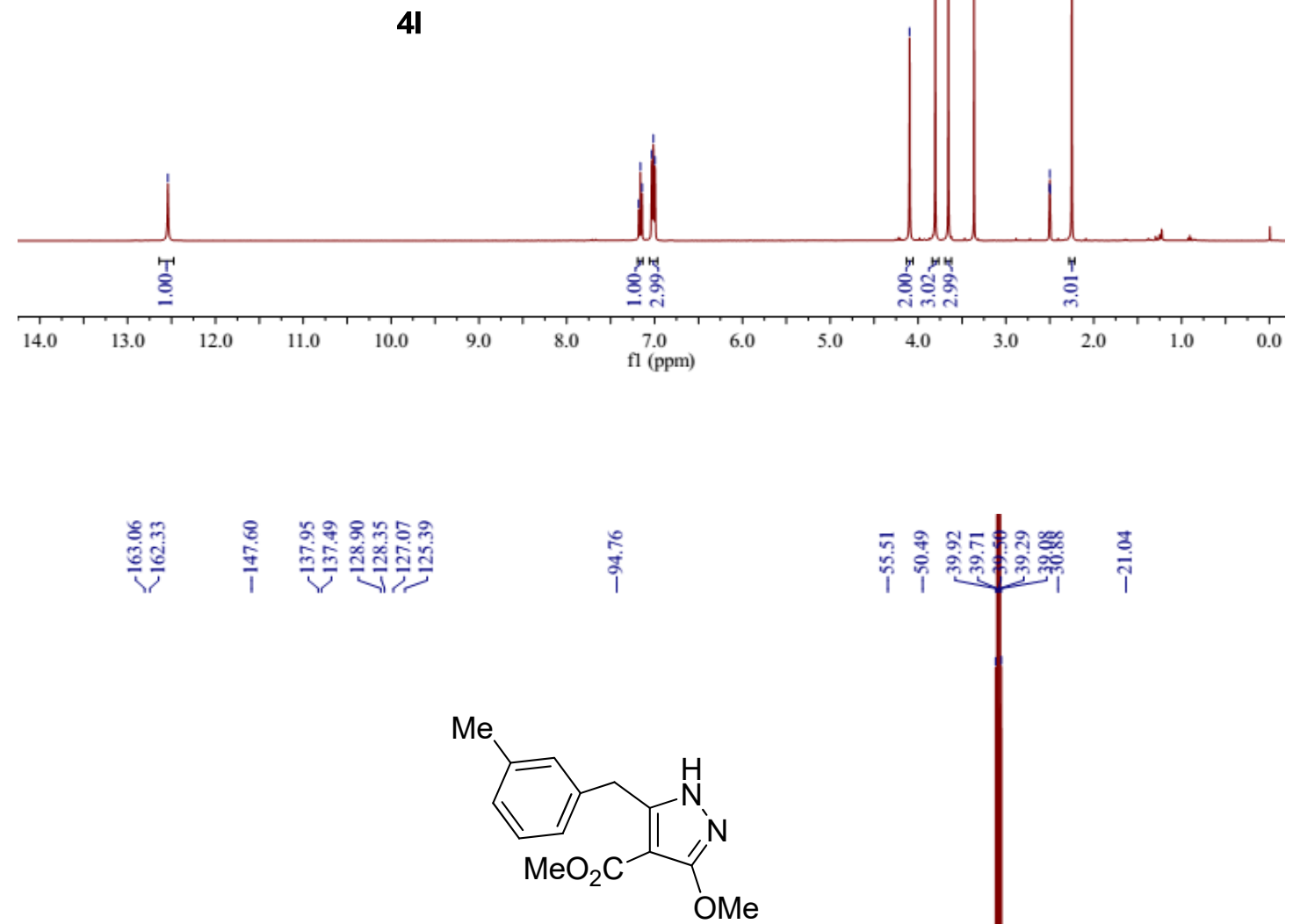

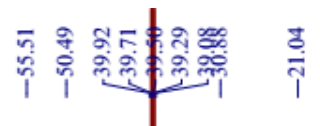

${ }^{13} \mathrm{C}$ NMR $\left(100 \mathrm{MHz}\right.$, DMSO- $\left.d_{6}\right)$

4I

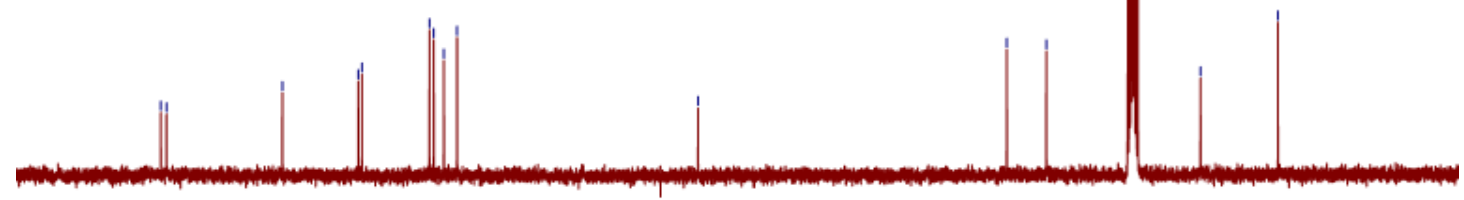

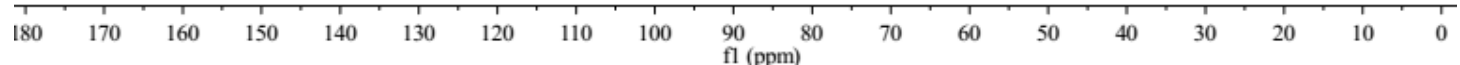



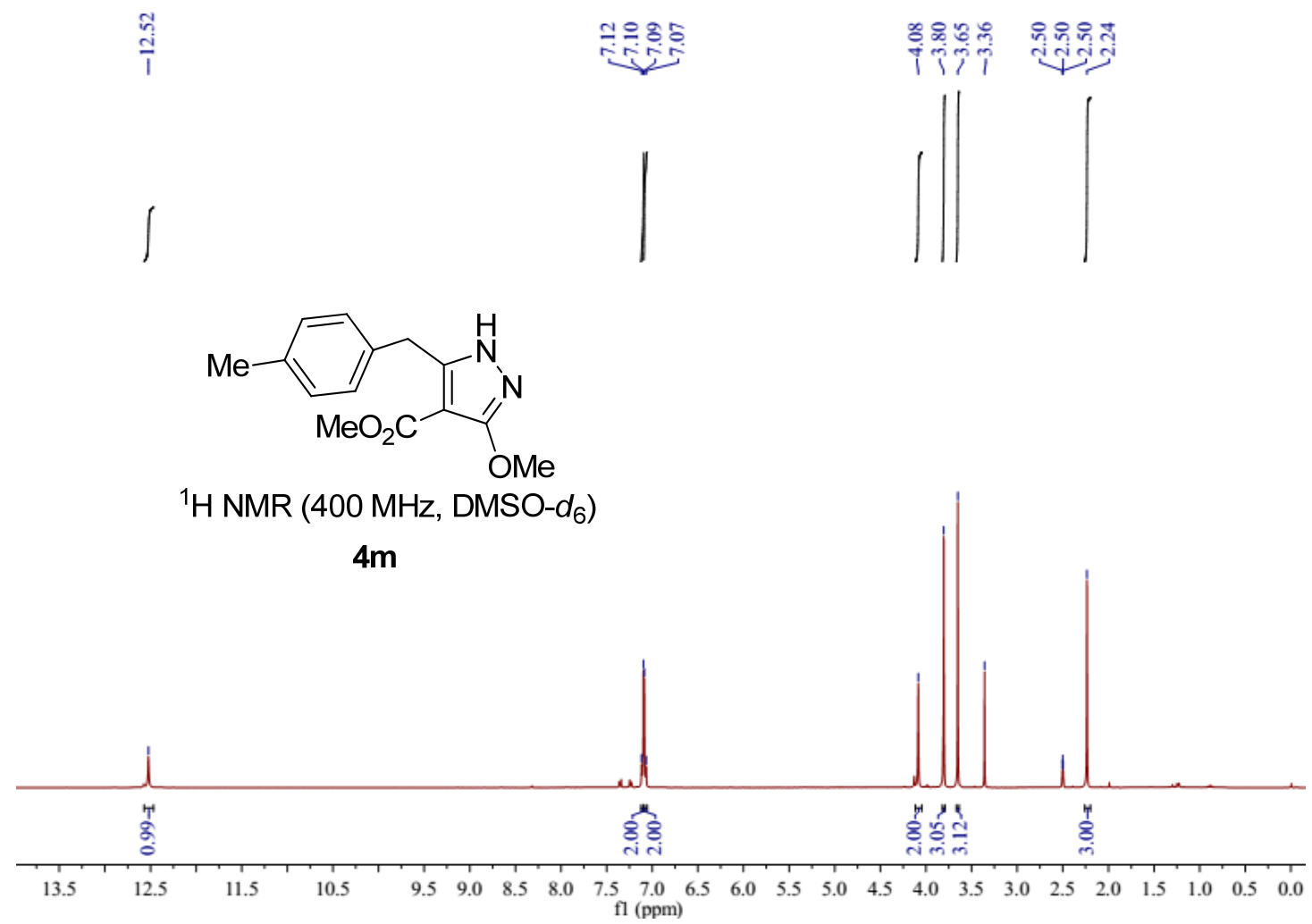

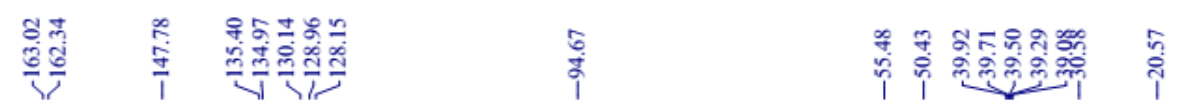

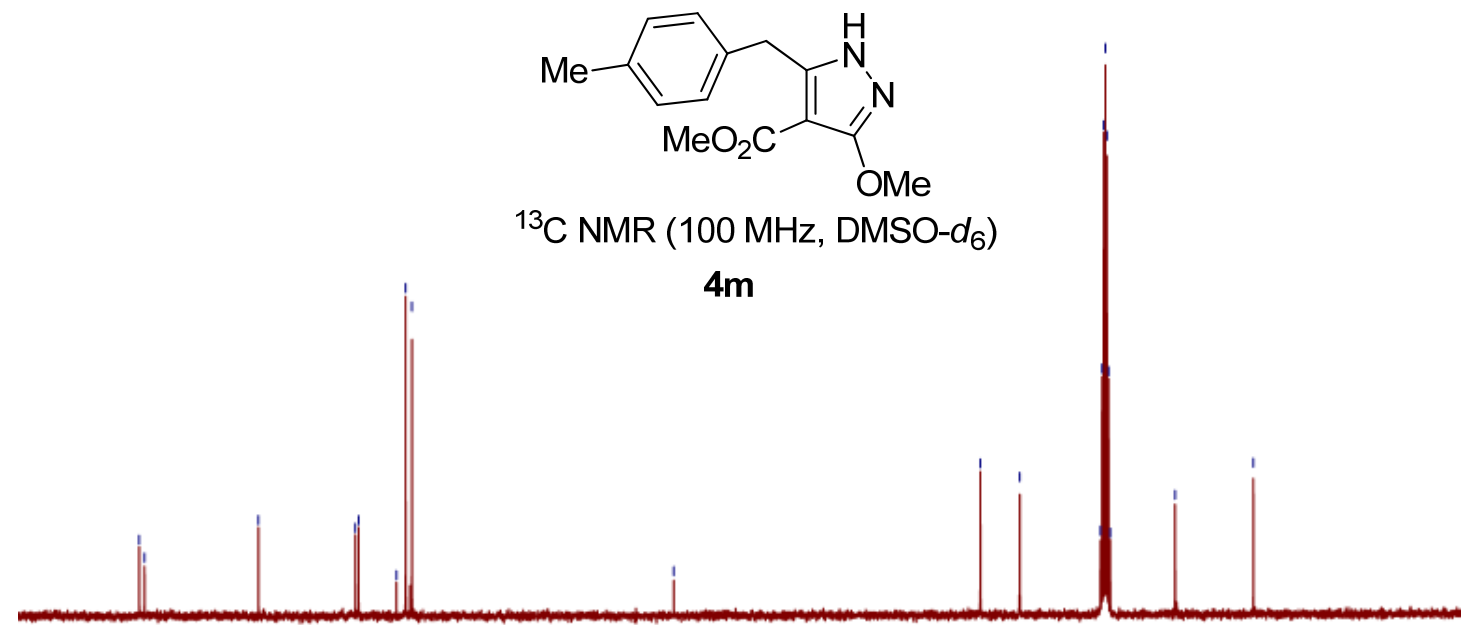

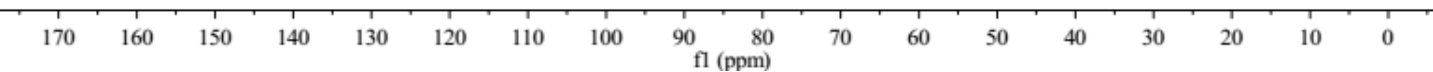


<smiles>CC#CC</smiles>
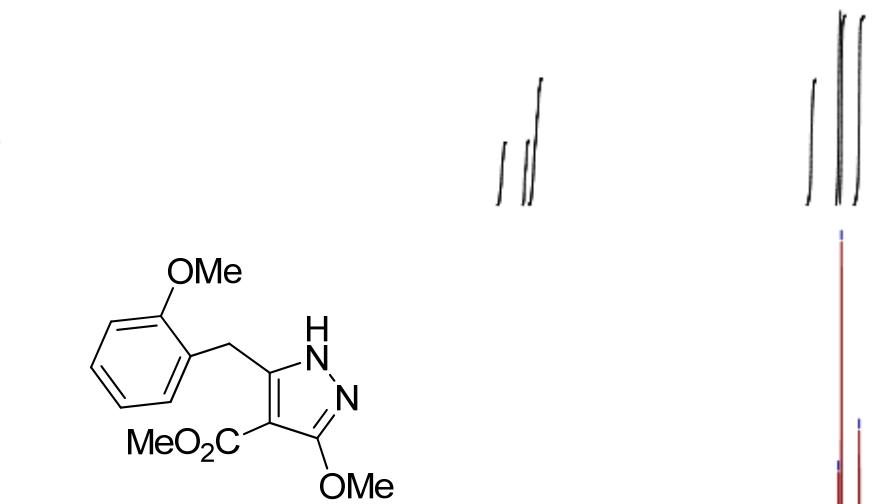

${ }^{1} \mathrm{H}$ NMR (400 MHz, DMSO- $d_{6}$ )

$4 n$

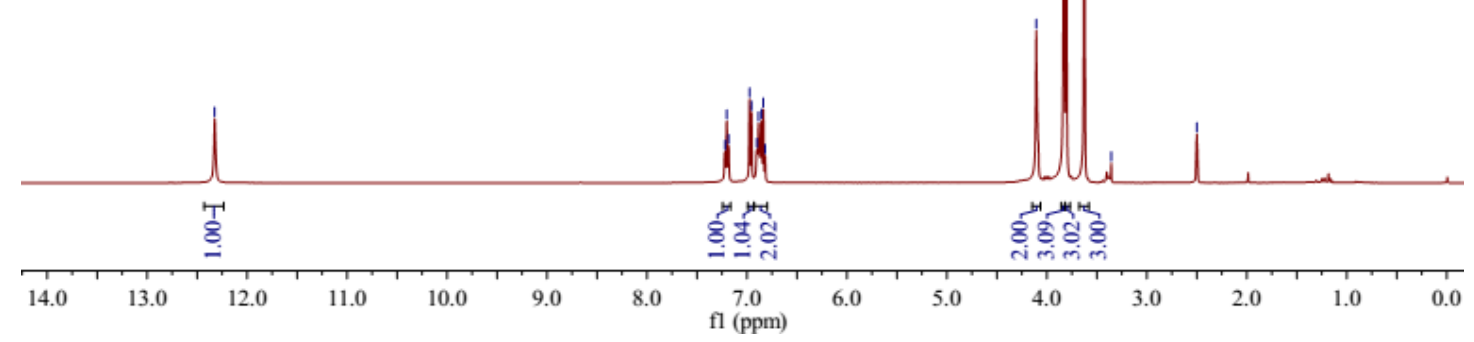

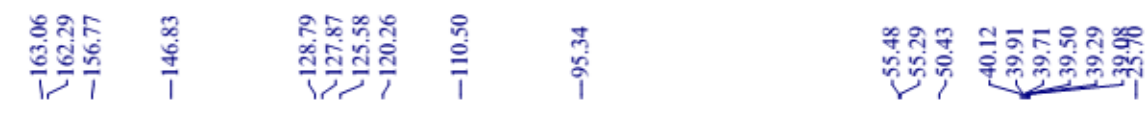

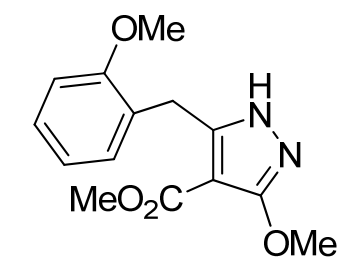

${ }^{13} \mathrm{C}$ NMR (100 MHz, DMSO-d $\left.{ }_{6}\right)$

$4 n$
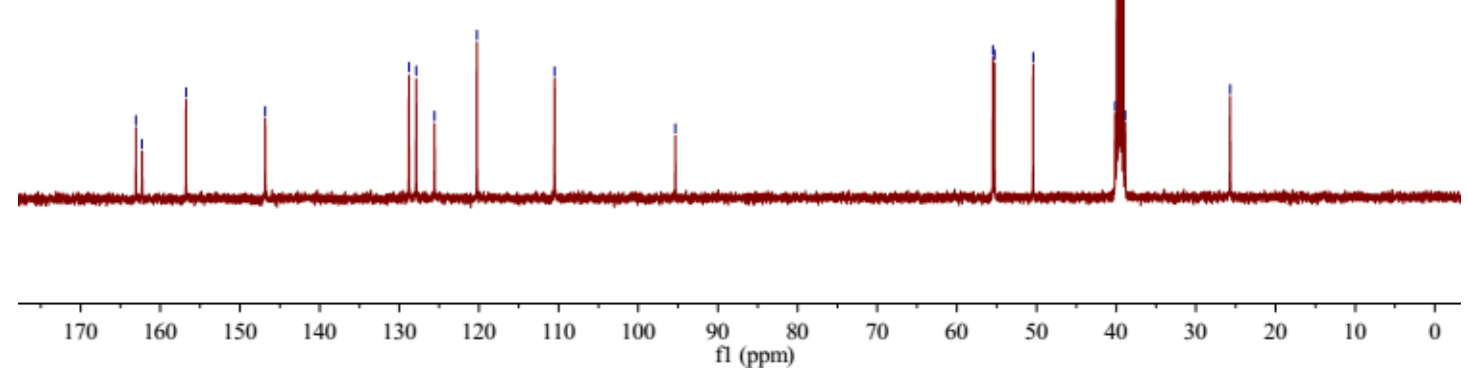

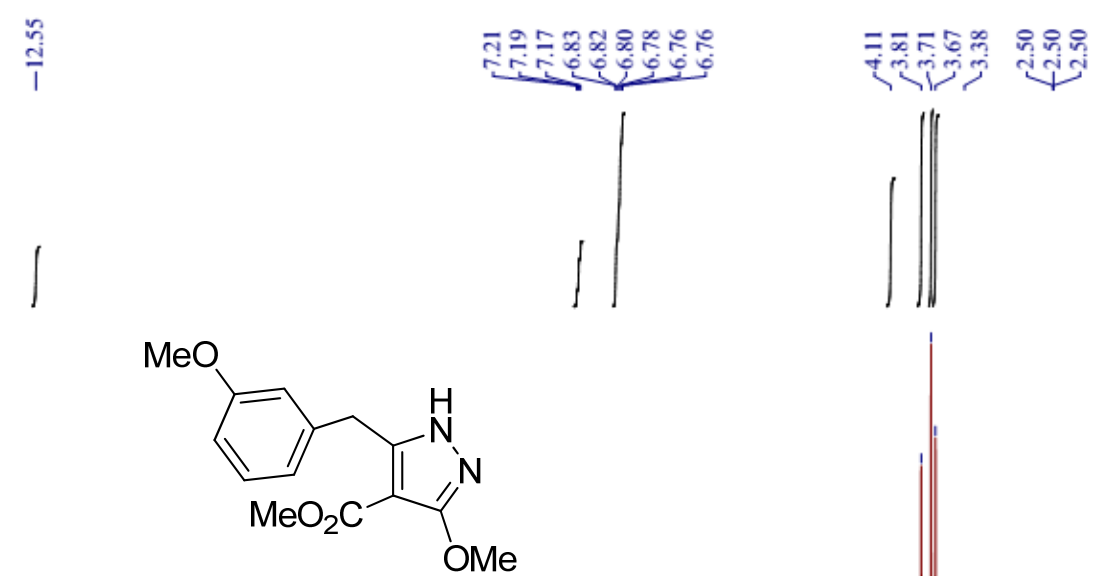

${ }^{1} \mathrm{H}$ NMR $\left(400 \mathrm{MHz}\right.$, DMSO- $\left.d_{6}\right)$

40
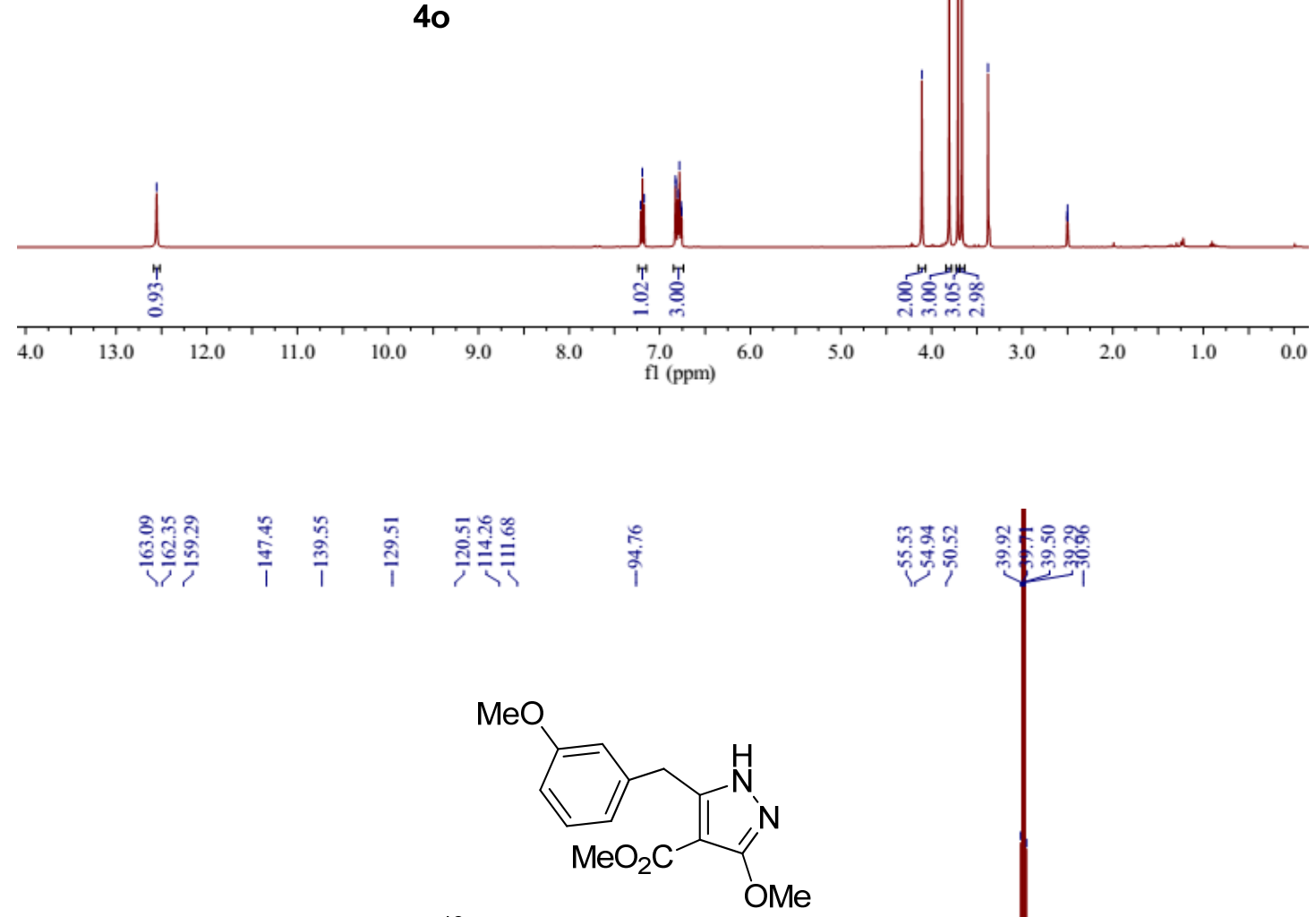

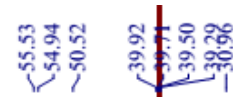

${ }^{13} \mathrm{C}$ NMR (100 MHz, DMSO- $\left.d_{6}\right)$

4o

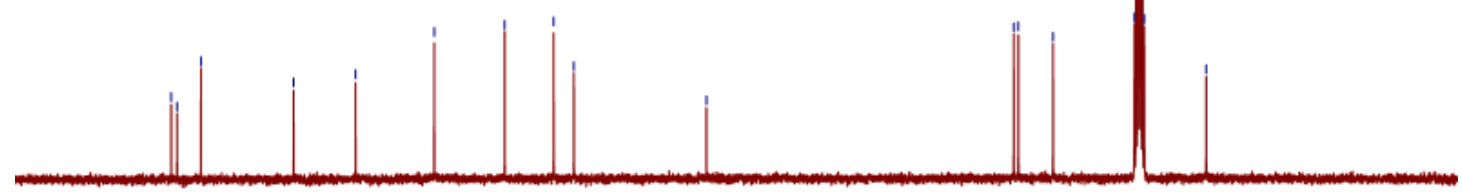

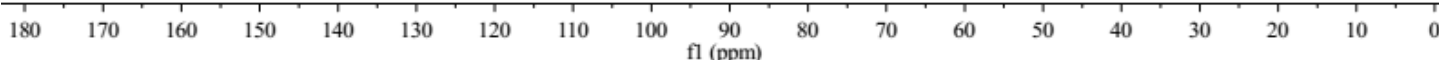



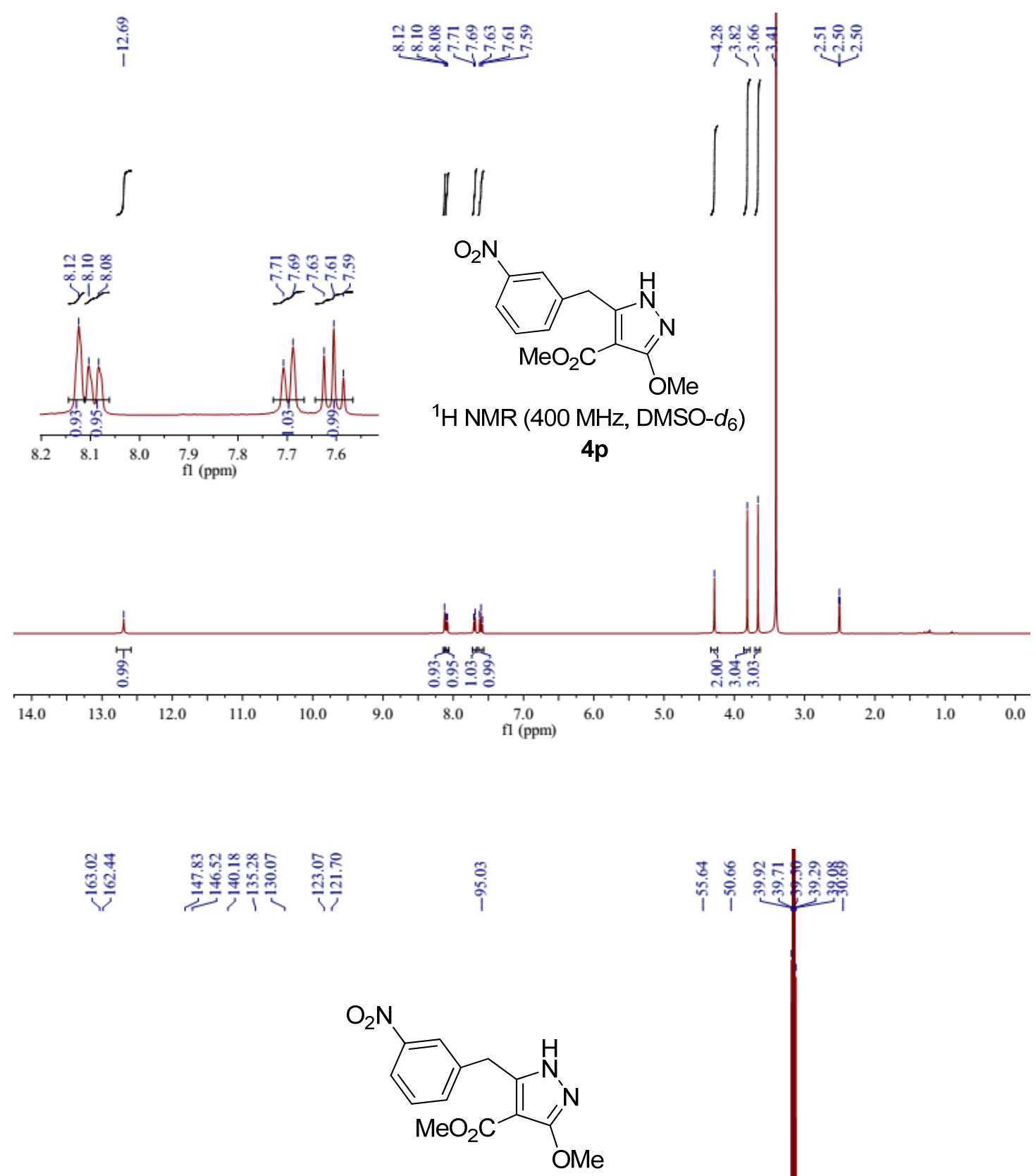

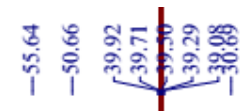

${ }^{13} \mathrm{C}$ NMR (100 MHz, DMSO- $d_{6}$ )

$4 p$

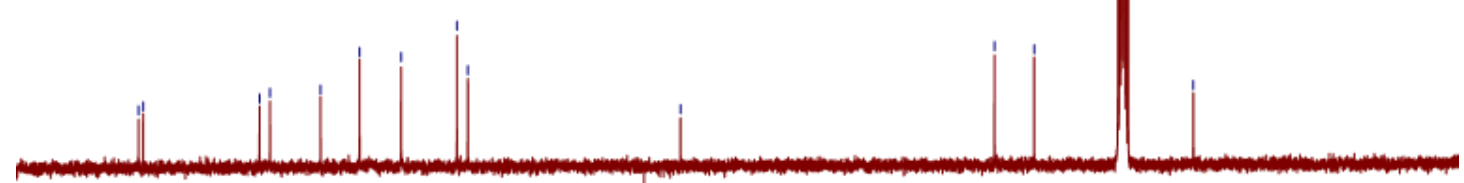

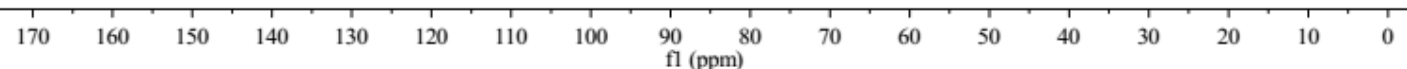



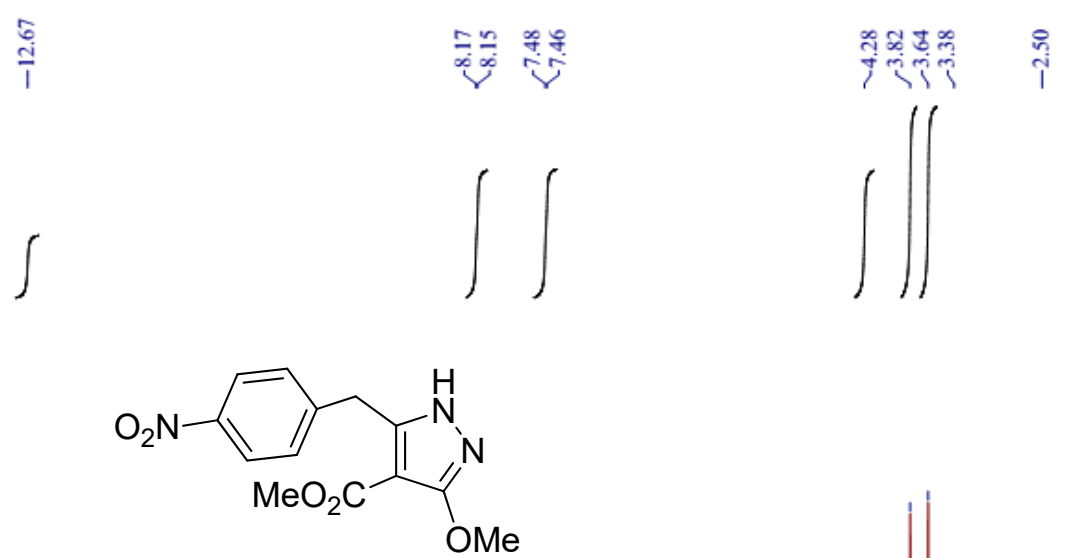

${ }^{1} \mathrm{H}$ NMR (400 MHz, DMSO- $d_{6}$ )

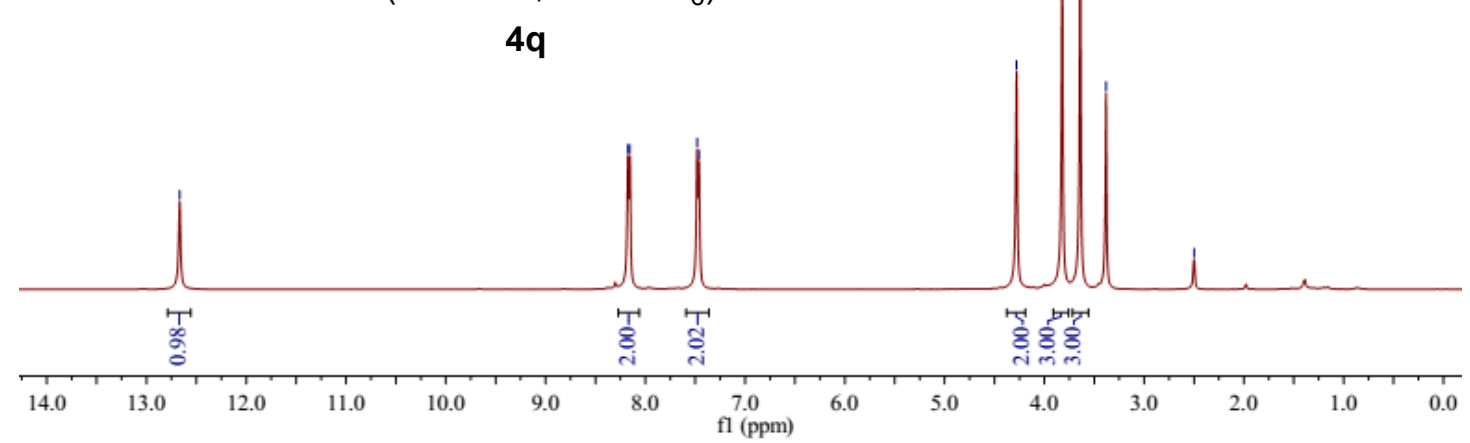

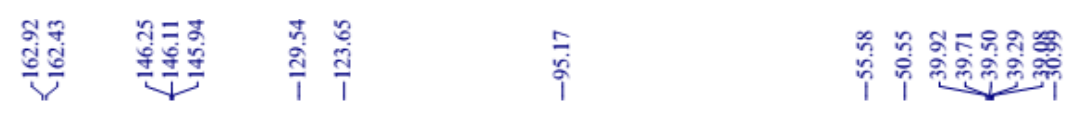
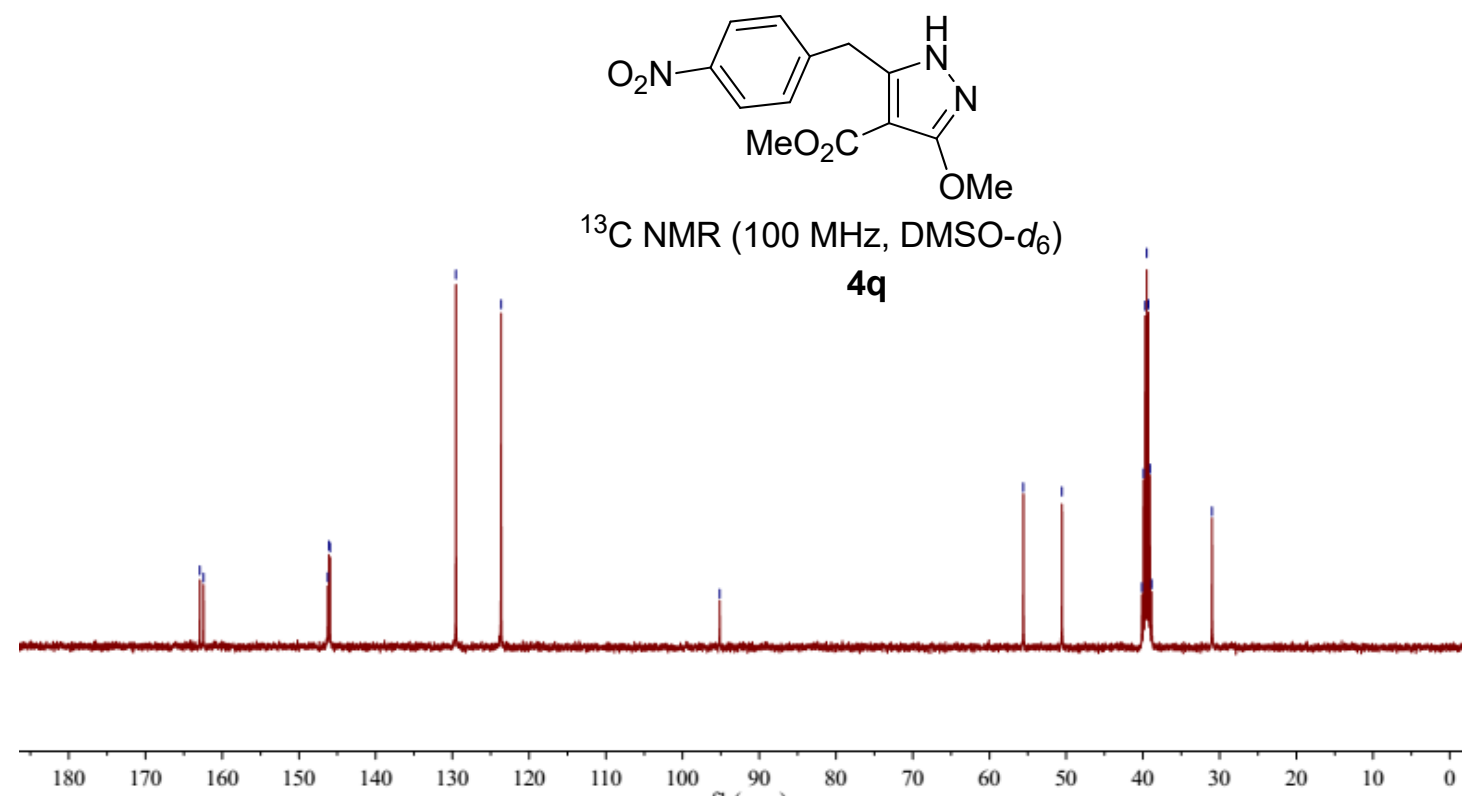


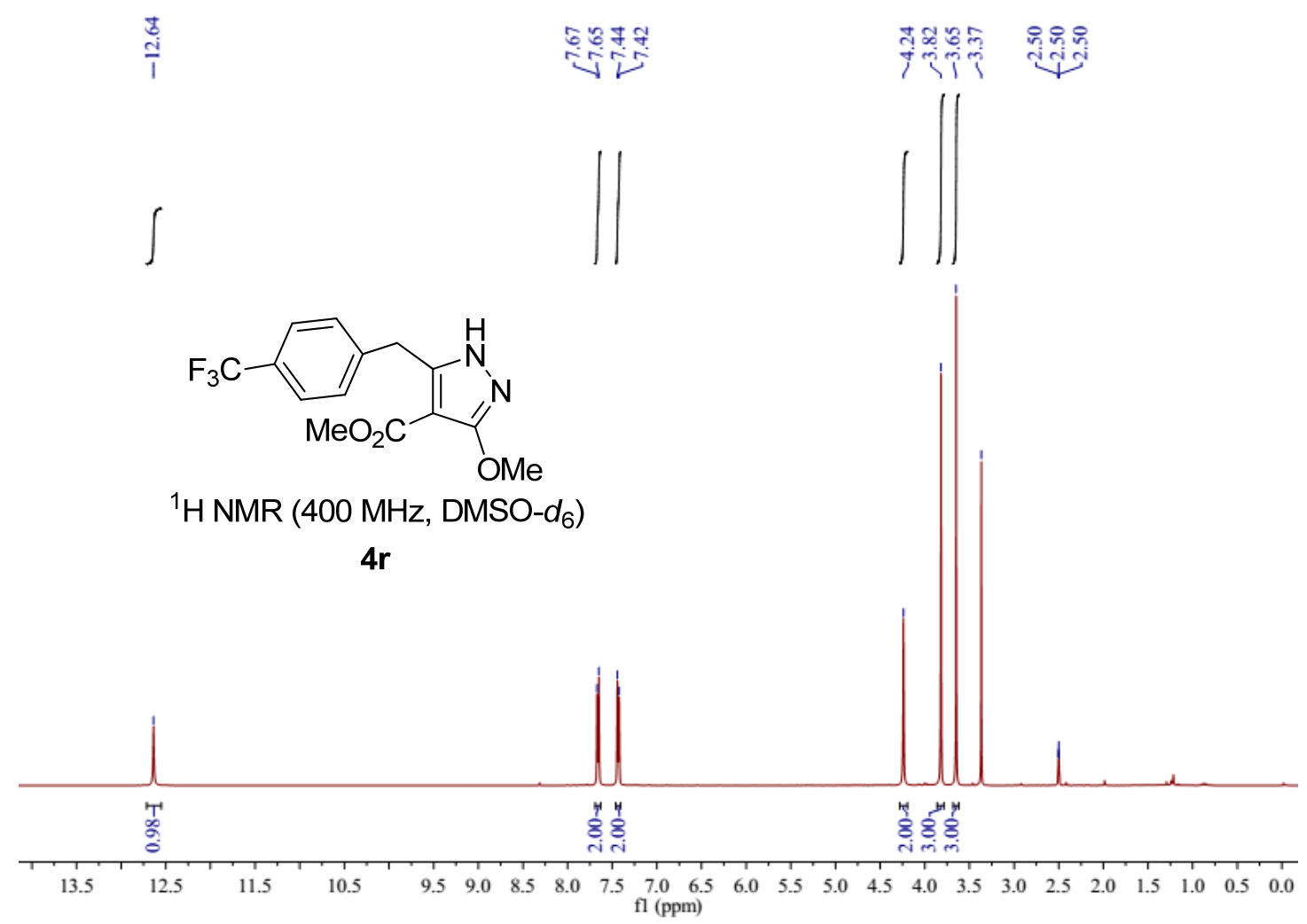

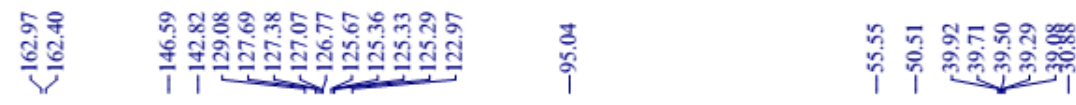

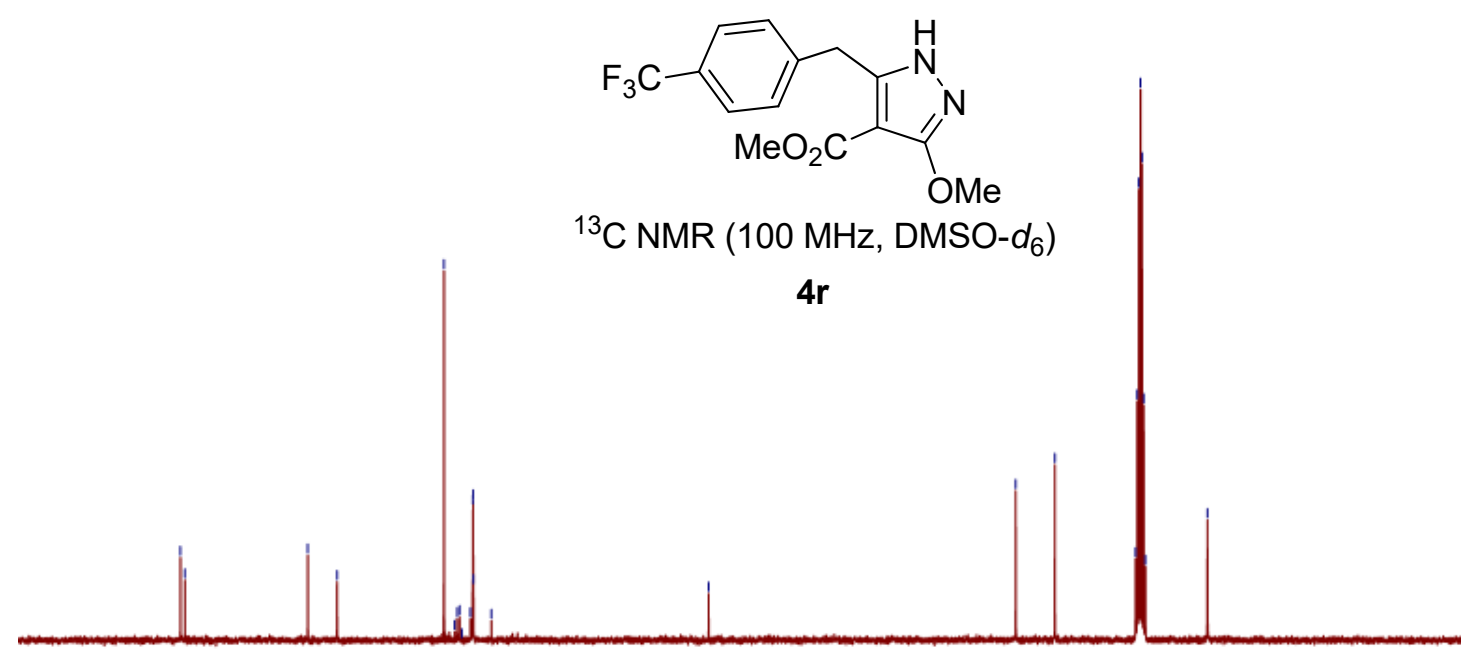

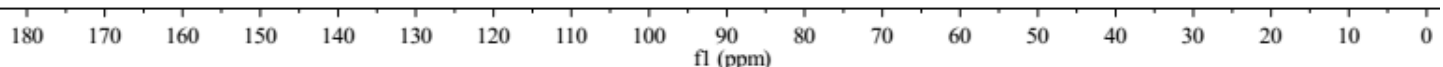




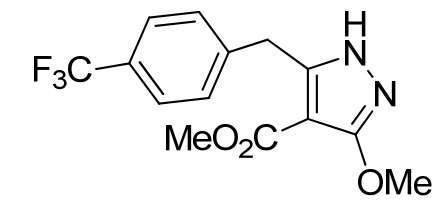

${ }^{19} \mathrm{~F}$ NMR (376 MHz, DMSO- $d_{6}$ )

$4 r$

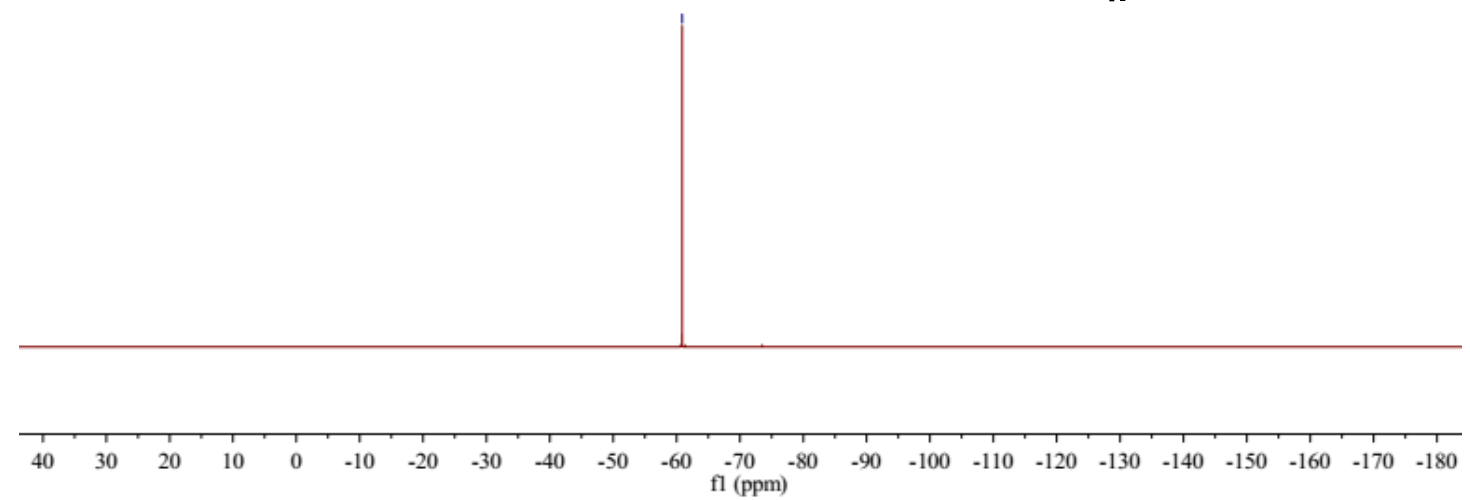

$\frac{1}{\text { a }}$

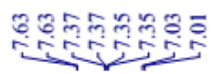

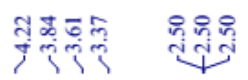

$\int$

$\iiint$

$\iint$<smiles>COC(=O)c1c(OC)n[nH]c1Cc1ccc(Cl)cc1Cl</smiles>

${ }^{1} \mathrm{H}$ NMR (400 MHz, DMSO-d ${ }_{6}$ )

$4 s$

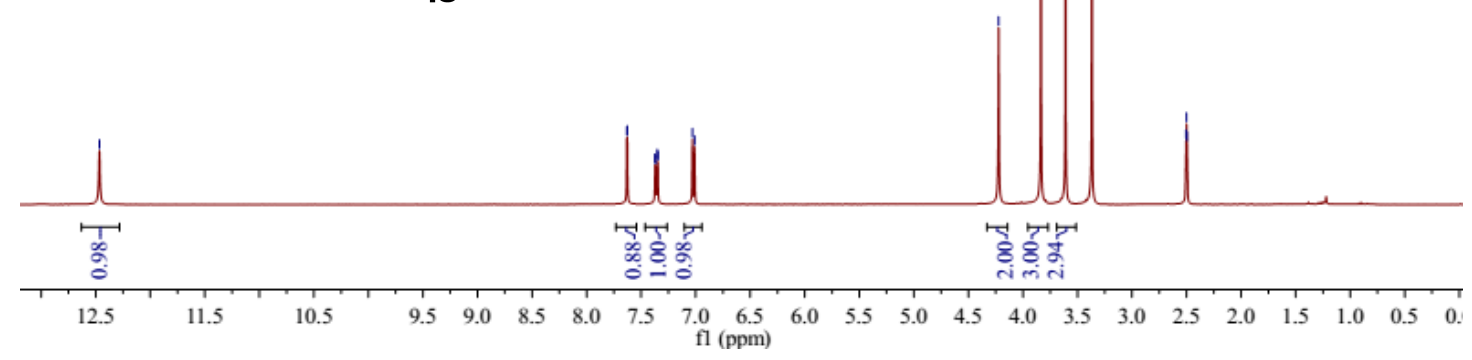



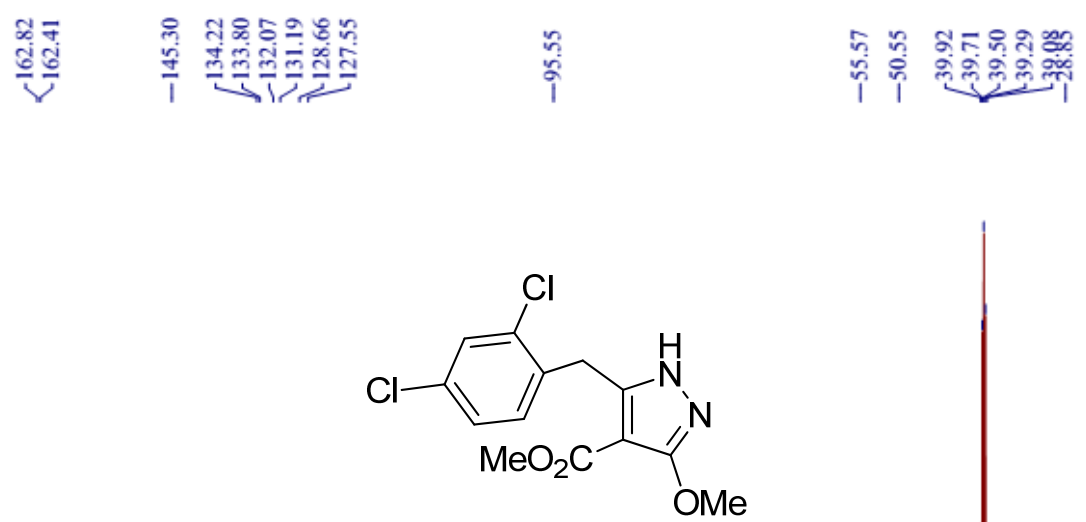

${ }^{13} \mathrm{C}$ NMR (100 MHz, DMSO-d 6 )

$4 s$
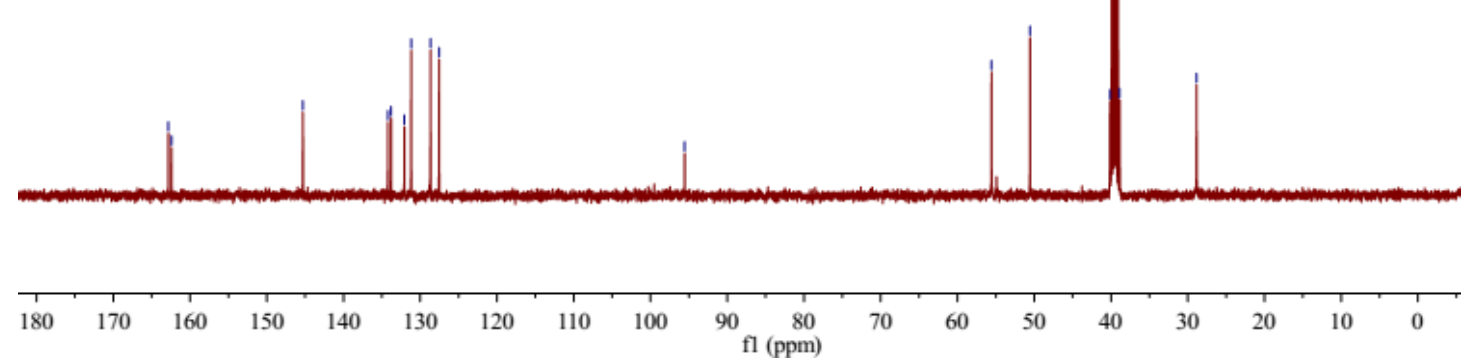

$\frac{g}{i}$

ชู

S

$\int$
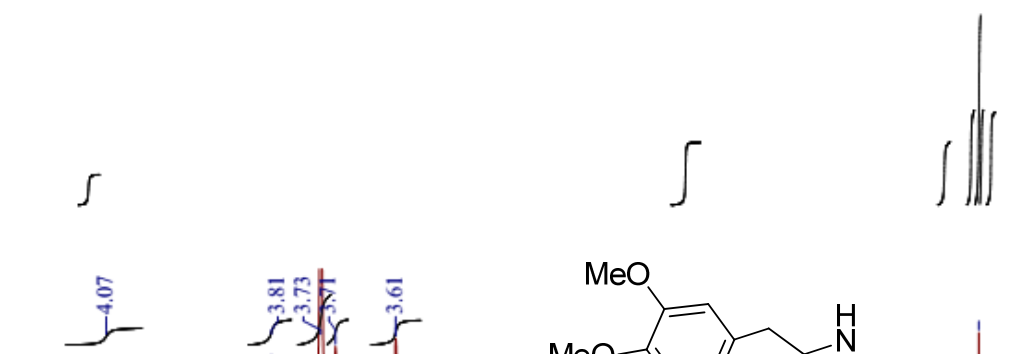

点
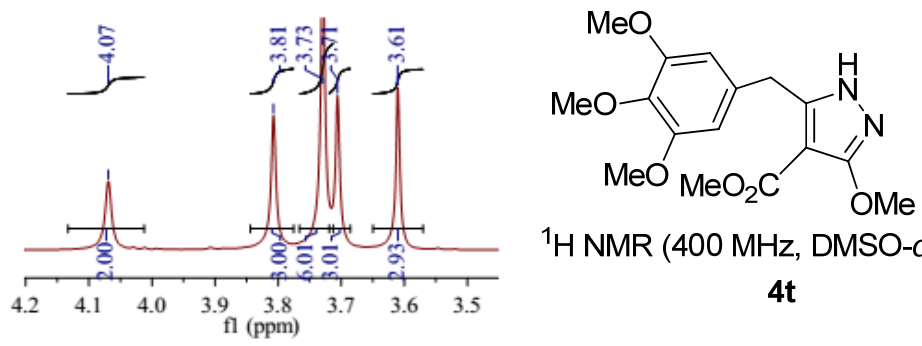

${ }^{1} \mathrm{H}$ NMR (400 MHz, DMSO-d $\left.d_{6}\right)$

$4 \mathbf{t}$

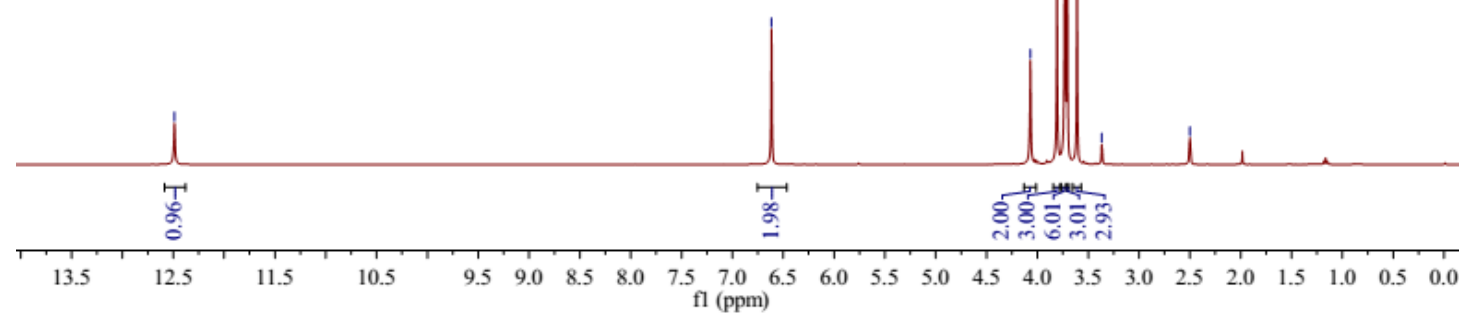



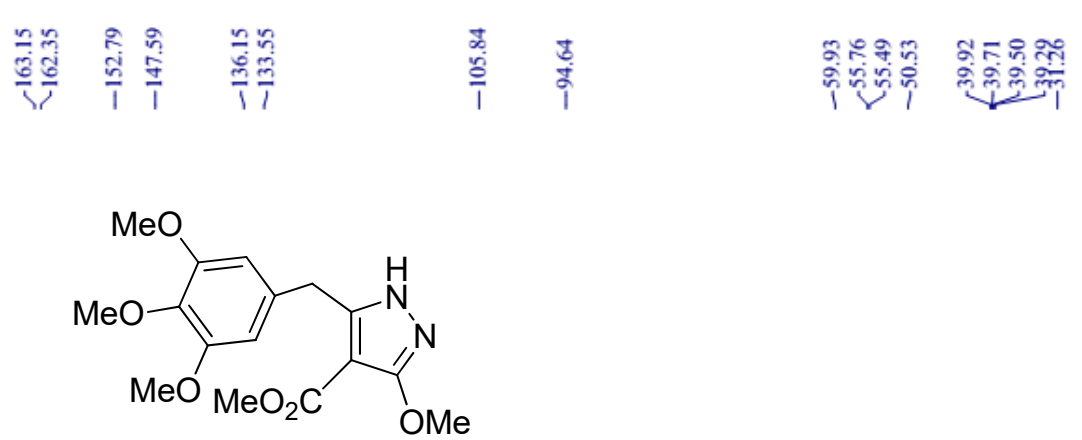

${ }^{13} \mathrm{C}$ NMR (100 MHz, DMSO-d 6 )

$4 t$

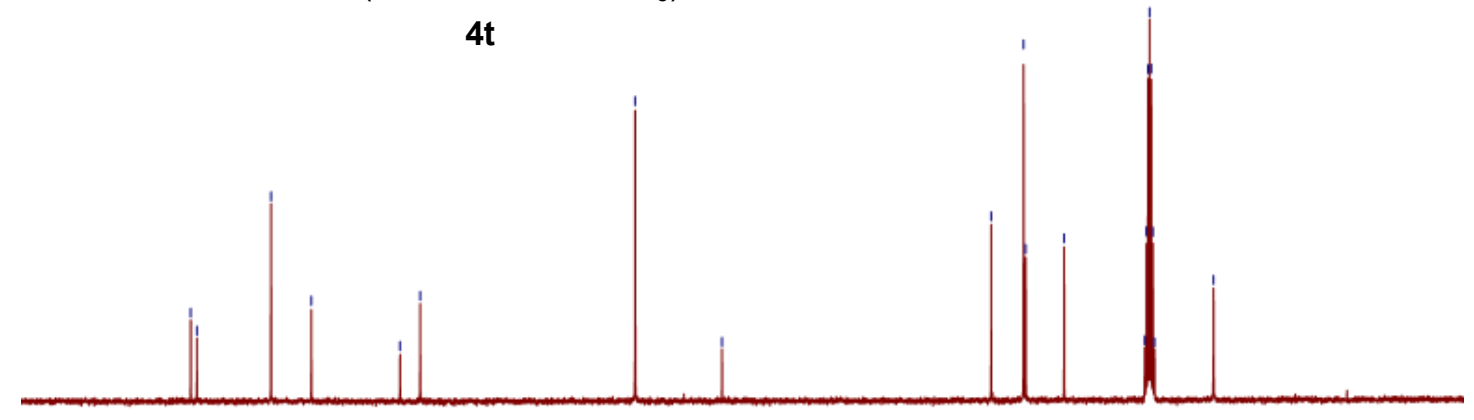

\begin{tabular}{|c|c|c|c|c|c|c|c|c|c|c|c|c|c|c|c|c|c|}
\hline 180 & 170 & 160 & 150 & 140 & 130 & 120 & 110 & 100 & $\begin{array}{c}90 \\
(\mathrm{ppm})\end{array}$ & 80 & 70 & 60 & 50 & 40 & 30 & 20 & 10 \\
\hline
\end{tabular}
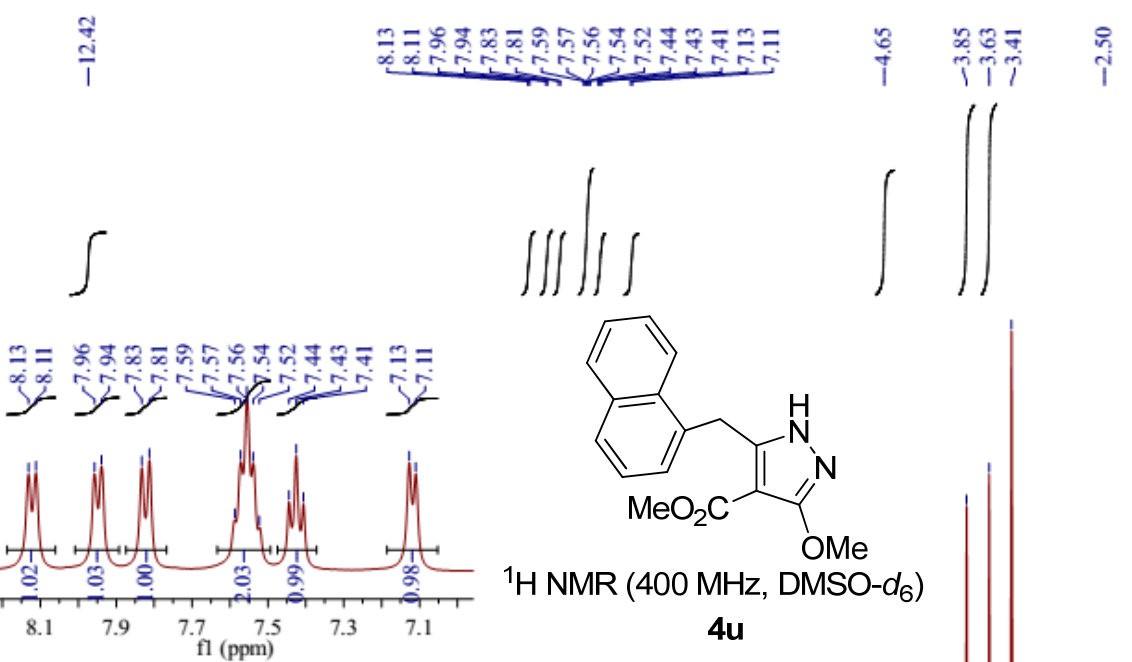

${ }^{1} \mathrm{H}$ NMR (400 MHz, DMSO-d 6 )

$4 u$

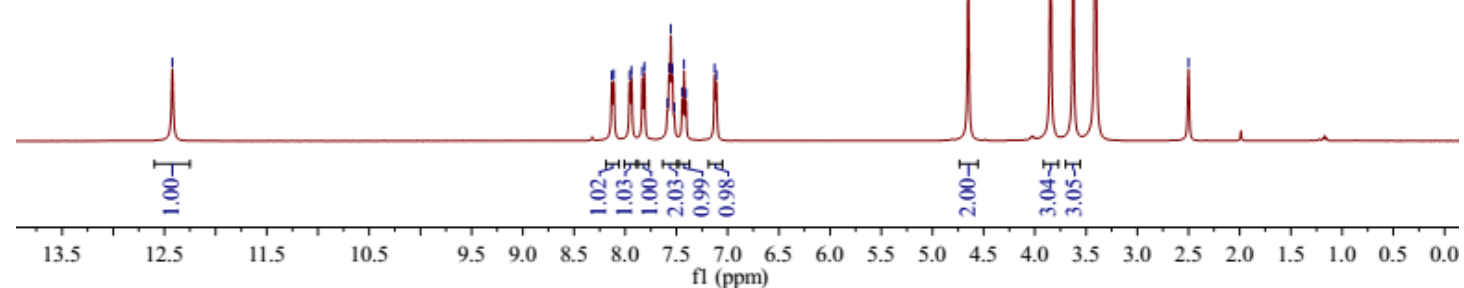




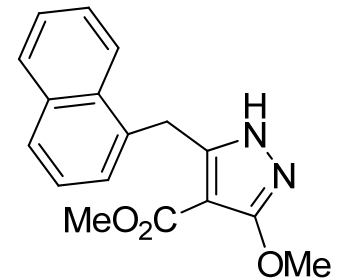

${ }^{13} \mathrm{C}$ NMR (100 MHz, DMSO- $d_{6}$ )

$4 u$
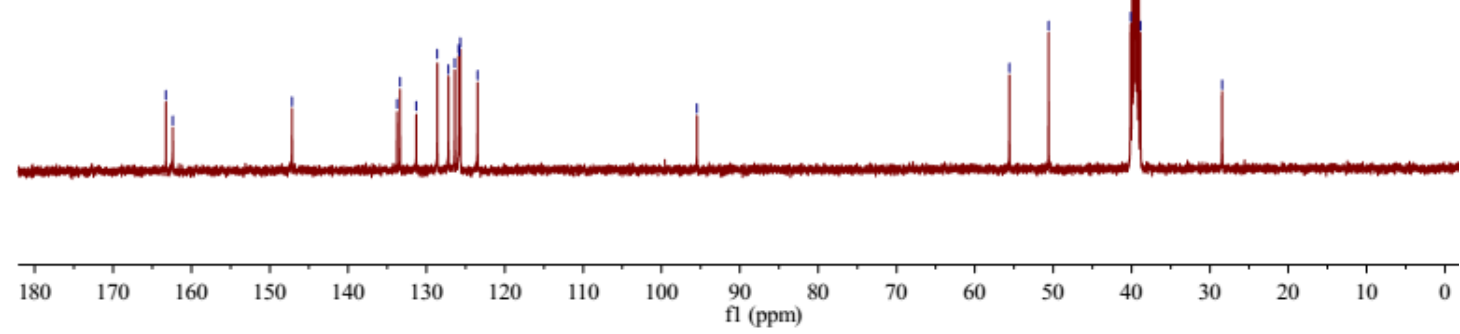

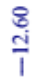

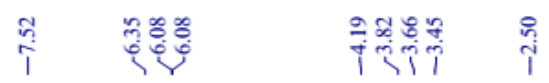

$\int$
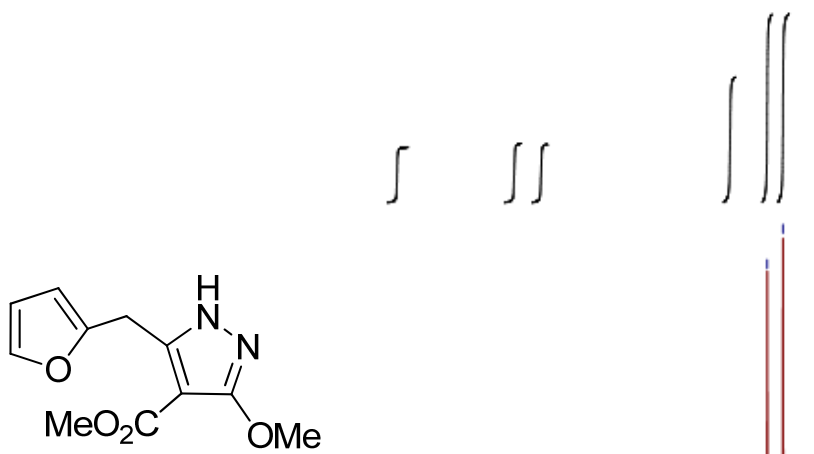

${ }^{1} \mathrm{H}$ NMR (400 MHz, DMSO-d ${ }_{6}$ )

4v

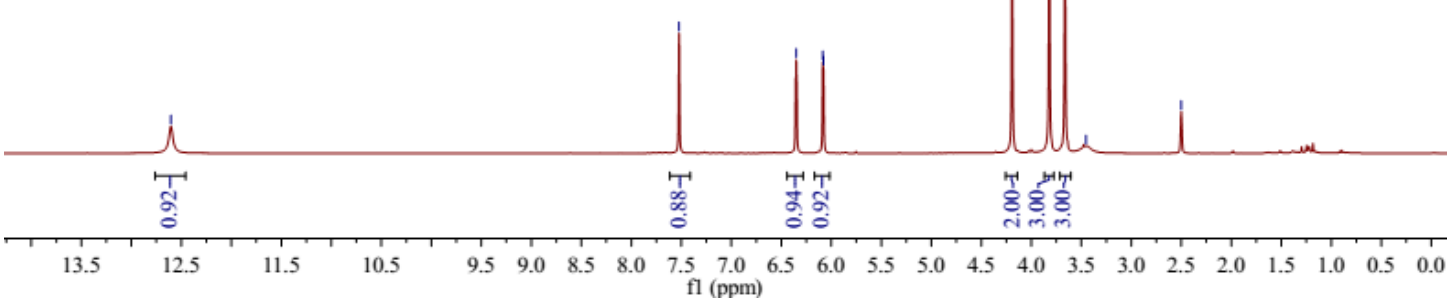



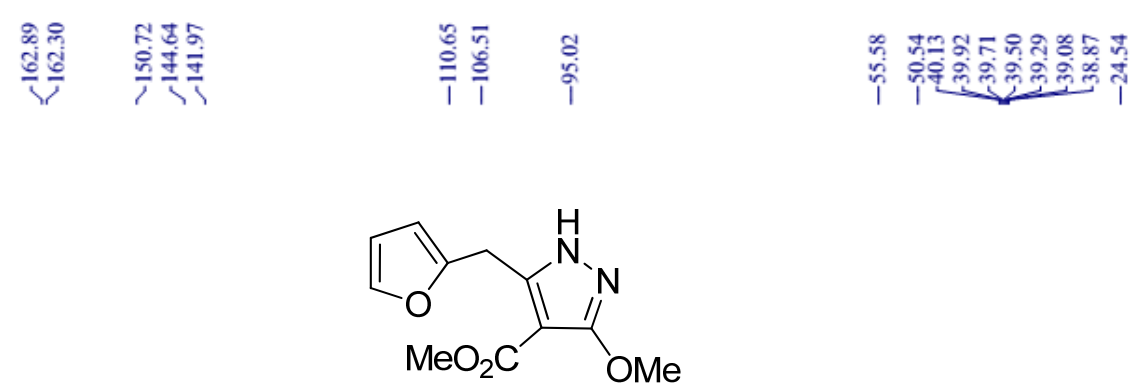

${ }^{13} \mathrm{C}$ NMR $\left(100 \mathrm{MHz}\right.$, DMSO- $\left.d_{6}\right)$

$4 v$
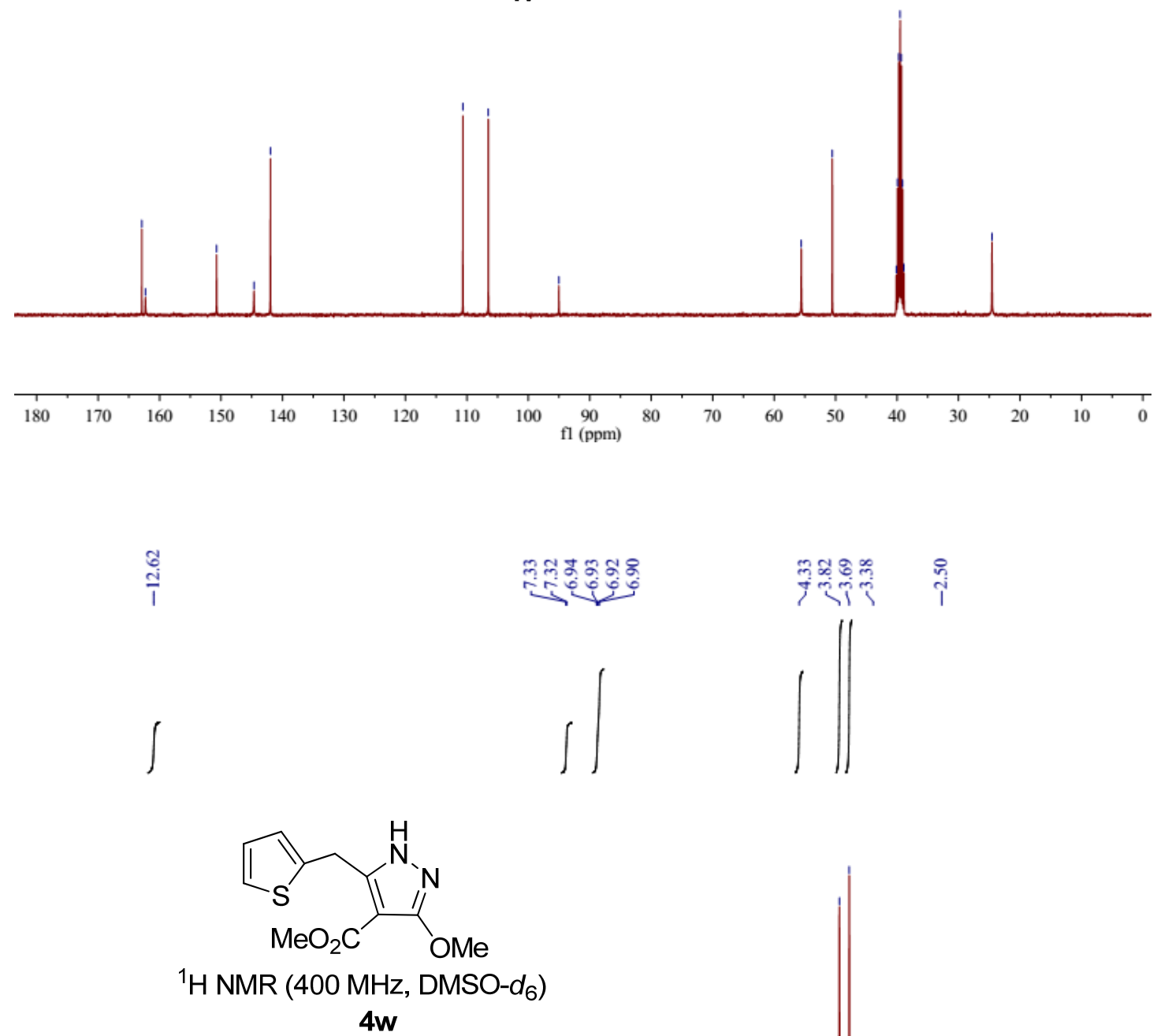


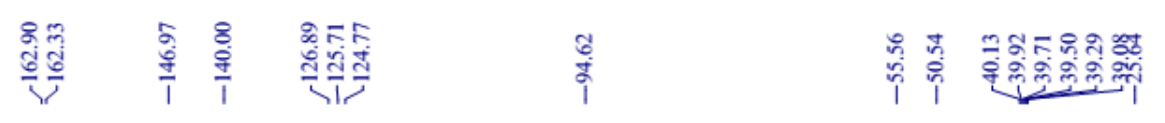
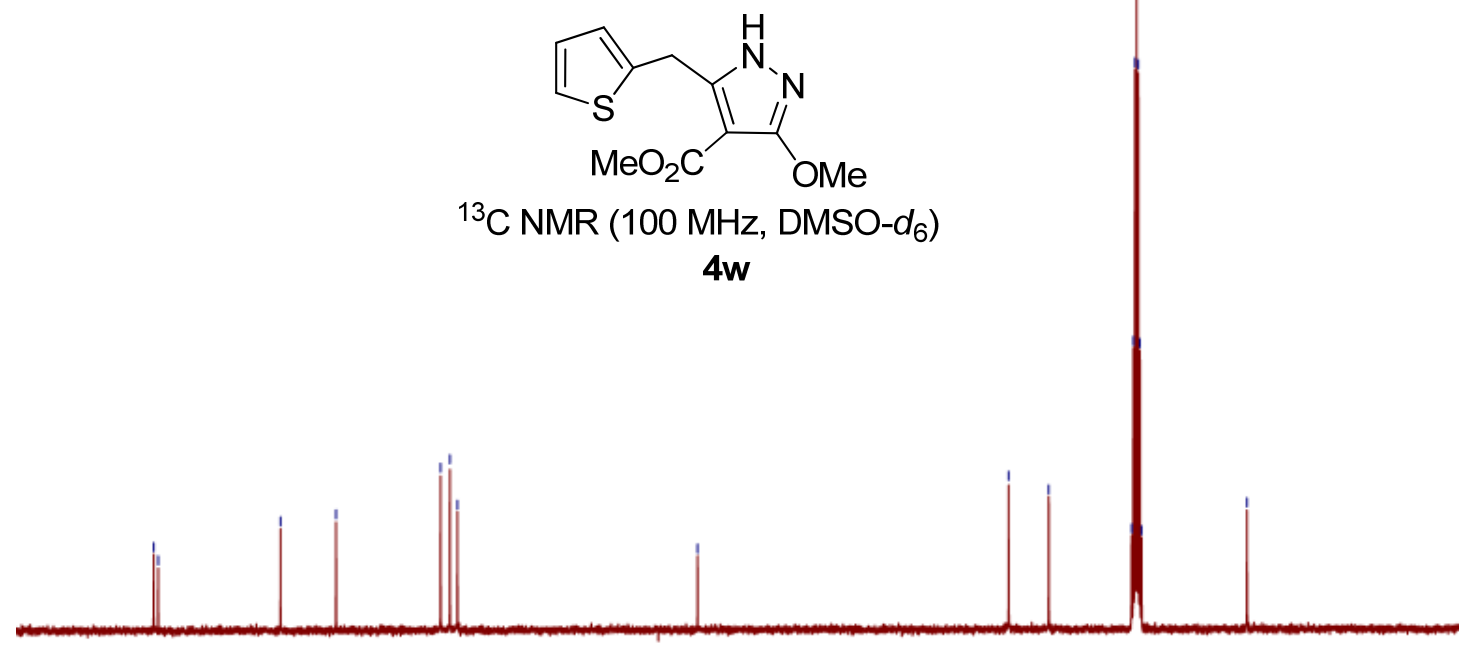

\begin{tabular}{|c|c|c|c|c|c|c|c|c|c|c|c|c|c|c|c|c|c|c|}
\hline 30 & 170 & 160 & 150 & 140 & 130 & 120 & 110 & 100 & $\begin{array}{l}90 \\
\mathrm{fl}(\mathrm{ppm})\end{array}$ & 80 & 70 & 60 & 50 & 40 & 30 & 20 & 10 & 0 \\
\hline
\end{tabular}

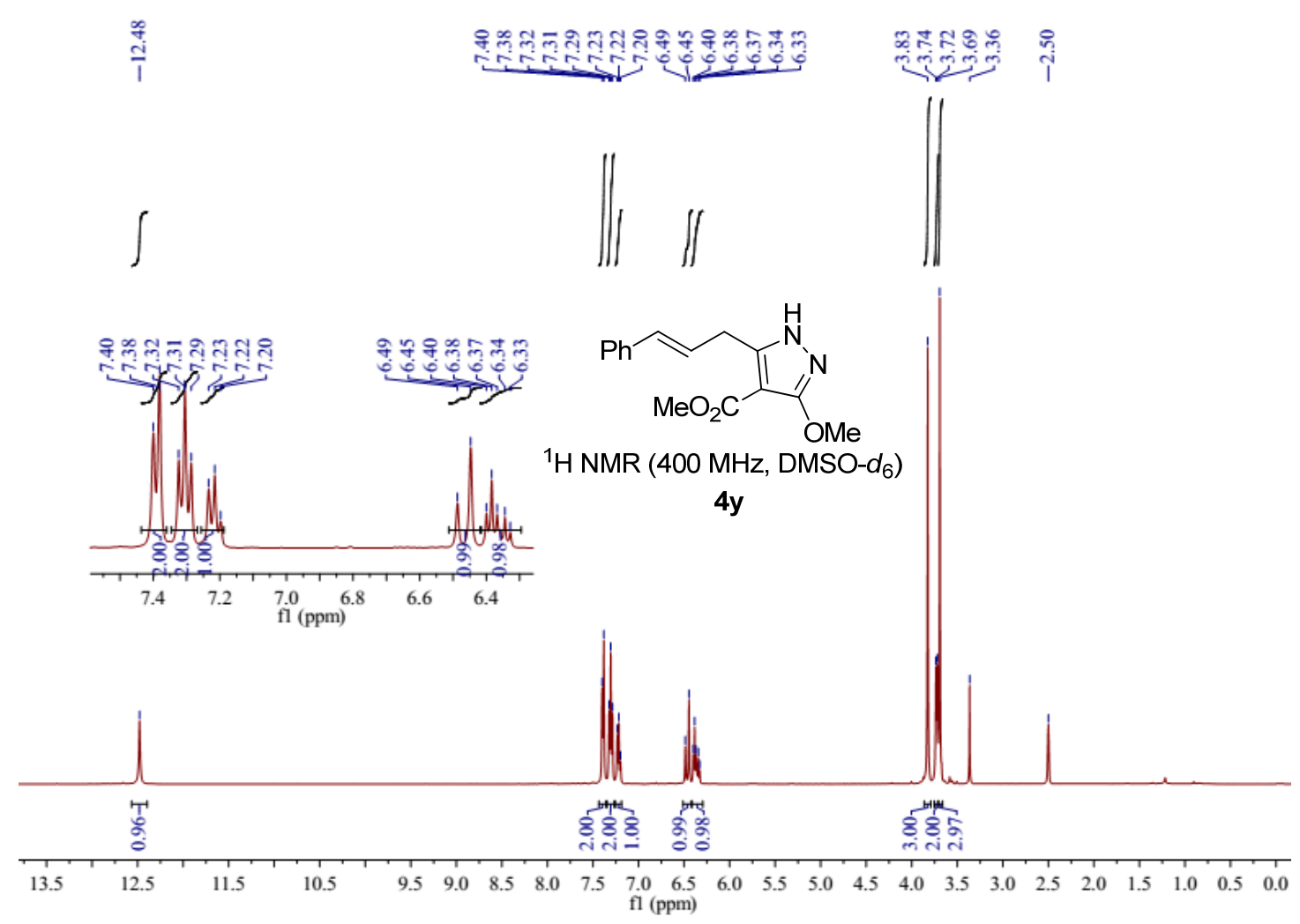




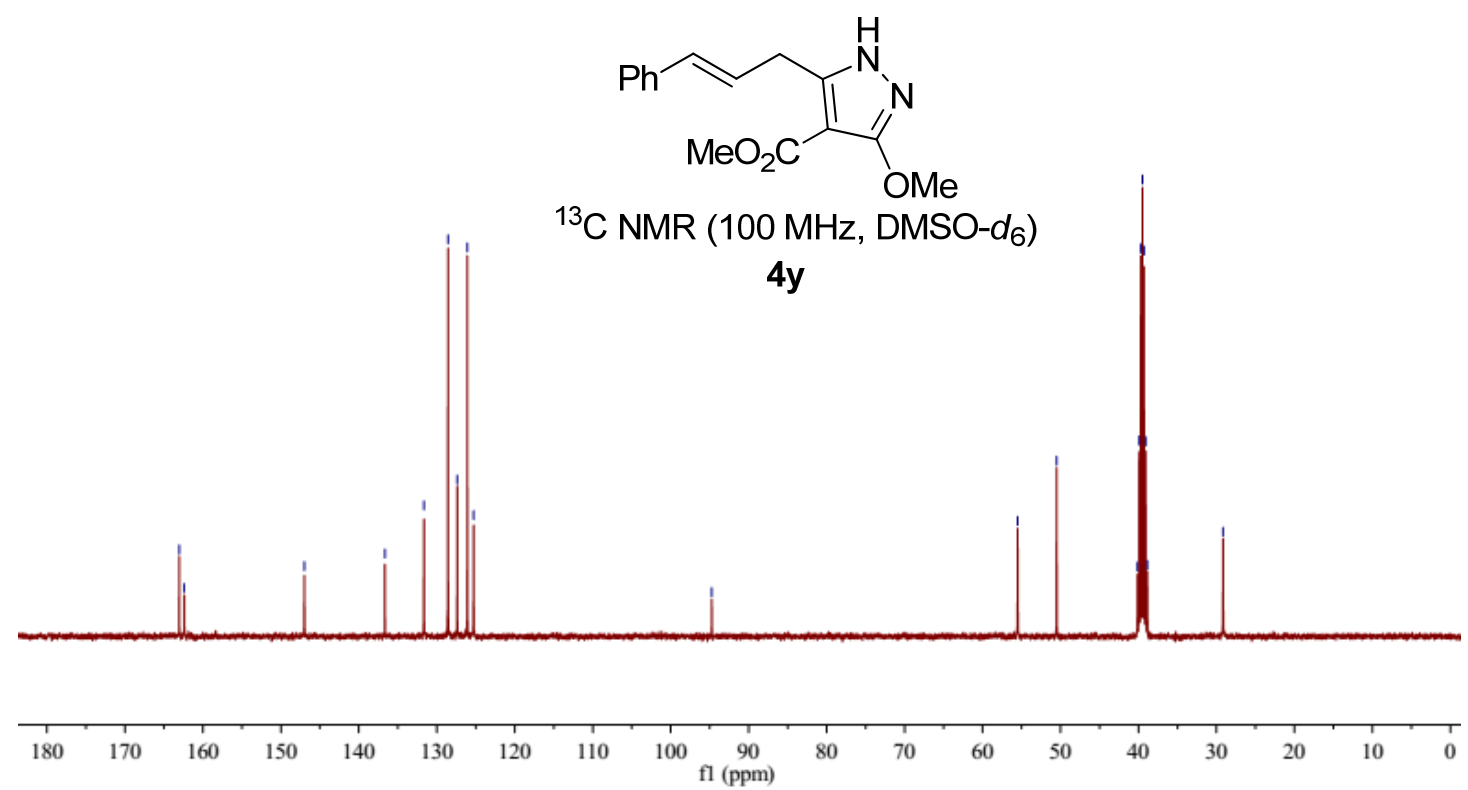

